\title{
Building a Better Atomic Clock
}

by

\section{Benjamin J. Bloom}

B.S., Massachusetts Institute of Technology, 2008

\author{
A thesis submitted to the \\ Faculty of the Graduate School of the \\ University of Colorado in partial fulfillment \\ of the requirements for the degree of \\ Doctor of Philosophy \\ Department of Physics
}

2014 
This thesis entitled:

Building a Better Atomic Clock

written by Benjamin J. Bloom

has been approved for the Department of Physics

Jun Ye

James Thompson

Date

The final copy of this thesis has been examined by the signatories, and we find that both the content and the form meet acceptable presentation standards of scholarly work in the above mentioned discipline. 
Bloom, Benjamin J. (Ph.D., Physics)

Building a Better Atomic Clock

Thesis directed by Prof. Jun Ye

Since 1967, the Second has been defined by the CGPM as 9,192,631,770 periods of oscillation corresponding to a transition between two hyperfine levels in a Cesium atom with zero external fields. Key to this definition was the fact that as experimentalists found new and more precise ways to measure this frequency, the definition of the second would become more and more accurate with time. However, in the last 30 years, new technologies based on tunable lasers addressing optical transitions in atoms, ions, and molecules have offered an entirely new approach to defining the Second with significantly higher precision and accuracy. Here, I will show that by trapping thousands of atoms inside a specially engineered optical lattice one can create an extremely accurate frequency standard with 2 orders of magnitude improvement over current Cs standards. Furthermore, I will explain that standards based on this technology are fundamentally more stable than the primary standard by 3 orders of magnitude. Leapfrogging the currently held accuracy records of ion clocks, this work documents the first optical lattice clock to best all other atomic clock implementations, a mere 8 years after the first proof of principle experiments. In this thesis I will describe how we have overcome a number of important systematic uncertainties to achieve these results. This revolution in accuracy and precision opens the door for new experiments utilizing the clock as a probe of quantum many-body physics. To this end, a new apparatus has been designed that combines the unprecedented precision of clock experiments with the amazing progress attained in quantum gas experiments. 


\section{Dedication}

To my family, both old and new. 


\section{Acknowledgements}

Before I go on to thank all the professors, students, and researchers who helped me achieve this PhD over the last few years, I first want to thank my family.

To my parents, I cannot imagine a more supportive and loving environment to grow up in. Whether I was making a mobile of the planets or building an R/C airplane with my dad, they allowed me to be me and supported me in every way.

To my brother and sister, whether or not you knew it you've always acted as a goalpost to me. Pushing me to work harder and achieve more, not just at work but in life too. Some of my happiest memories are of the three of us on vacation hysterically laughing at god only knows what.

To Ruth, I don't think I could have done this without you. The strength and love you have shown me has finally made me realize where my new home is.

To Max, thank you for making me realize how any problem can be overcome with boundless enthusiasm. To Einstein, thanks for coming back from your walkabout.

When I first showed up to JILA, Jun was a giant. His amazing work and reputation were all that I knew. But over the last 5 years, I've come to learn that exceeding his successes in the lab is his good nature and enthusiasm for Science! As all the research documented in this thesis and others will attest to, our projects meander towards their destination tacking left and jibing right as the grad students and postdocs make daily decisions. But at least once a week, Jun corrects our course and steers the ship back to our destination. Jun's mastery of experiment and complete knowledge of all things 'A', 'M', and 'O' are an inspiration for everybody who works with him.

I also owe a great heartfelt thanks to my undergraduate advisor Vladan Vuletíc who intro- 
duced me to the world of AMO, the most DIY discipline I have ever come across. His hilarious combination of Comic Sans, Oscar Wilde quotations, and Clifford Algebras enriched everyday in lab as an undergraduate. Over the last 7+ years, since stepping into Vladan's lab at the CUA, I've come to depend on Jon Simon's blend of camaraderie and mentorship. My circuitous path to JILA actually began with a year at Cornell. But after having a change of heart I went back to the field of AMO. Throughout that year though I got to spend the summer at CERN working on CMS with Prof. Peter Wittich and volunteer in the new labs of Prof. Mukund Vengalattore. But what I missed upon leaving Cornell and joining the Ye group were my first year graduate classmates at Cornell in the Fall of 2008. Slogging through the problem sets and the grading together, the walks to CTB, and waiting for what seemed like hours for our pizza to come at The Nines was all made fun by the shared misery of graduate school.

The Sr3 (or publicly the SrII) experiment was comprised of Sebastian Blatt, Travis Nicholson, and Gretchen Campbell. After a few months Gretchen left for her new job, and except for the occasional visit from Jan Thomsen, it was just us three. What I remember most from these first two years were the long days and nights of work, ending either with hair brained schemes like baking vacuum parts in somebody's apartment at 2 am, or driving out in a snow storm to get a drink from Conor O'Neill's. As the Jolly German, Sebastian, was winding down his $\mathrm{PhD}$ we were joined by Jason Williams our new Postdoc. His good spirits and hilarious tales about his endless menagerie of pets helped us push along through the switch to clock mode. Following that Sara Campbell joined the fun, building the burninator Ti:Sapph which turned out to be so key in achieving our clock goals. Her exuberance for 'shredding the gnar', doing science, and endangering her own life will no doubt lead the new experiment to greatness :) . And most recently Ross Hutson and Reese McNally joined the team. The sky's the limit for the new apparatus and with Sara, Ross, Reese, and Jun leading the charge. I look forward to reading about all their future results.

None of the work in this thesis would be possible without the efforts of Mike Martin, Matt Swallows, Mike Bishof and Yige Lin. Without the help of these clock experts no SrII would exist today, especially Matt Swallows, who answered every dumb question I asked him. The new 
generation of SrI, Mike Bishof, Xibo Zhang, and Sarah Bromley, pushed to create what was at the time the second best reported optical lattice clock so that a clock comparison was possible. The future is bright for the latter two as they bring up their new cavity system while Mike leaves for his new job. Wei Zhang joined strontium from the combs side of thing and made our comparisons possible as the new laser guru. His new silicon cavity will one day revolutionize the clock community.

Trying to list all of the Ye labs members that have contributed to my projects by lending a helping hand or answering questions would be impossible. Needless to say, thank you to all Ye labs members past and present. (Including the honorary ones like Jan Hall!) Special thanks should also be extended to Judah Levine who helped us get an accurate Cs signal to lock our comb to. It should go without saying, this work has benefited in more ways than I can count from the dedicated professionals in the electronics and machine shop. I especially want to thank Hans Green for his work on the temperature sensors, Blaine Horner for his work on the new chamber, and Carl Sauer for not only working on all the projects I gave him but also being my goto electronics guy.

Finally, special thanks needs to goto Aviv Keshet, for his chamber pumping designs, the people at Sharon Vacuum including Larry Resnick for their excellent machining skills, Tim Darby at UKAEA, Tom Loftus at AOSense for his help with the new oven and Zeeman slower, and the researchers at Jefferson Labs who baked and coated our new chamber Philip Adderley, Marcy Stutzman, and Matt Poelker. 


\section{Contents}

\section{Chapter}

1 Introduction $\quad 1$

1.1 Optical Lattice Clocks . . . . . . . . . . . . . . . . . . . . . . . 2

1.1.1 The JILA ${ }^{87}$ Sr Lattice Clock . . . . . . . . . . . . . . . . . . . . . . 9

2 Building a Clock $\quad 12$

2.1 The Blue Laser System . . . . . . . . . . . . . . . . . . . . 12

2.1 .1 The Old Blue laser System . . . . . . . . . . . . . . . . . . . . 13

2.1.2 New Additions to the Blue team . . . . . . . . . . . . . . . . . . . 14

2.1 .3 The Blue Diode System . . . . . . . . . . . . . . . . . . 16

2.2 The Magic Wavelength Buildup Cavity . . . . . . . . . . . . . . . . 21

2.2 .1 A Cavity Autolocker . . . . . . . . . . . . . . . . 26

2.3 Spectrally clean light . . . . . . . . . . . . . . . . 33

2.4 Easy-to-build DDS Systems . . . . . . . . . . . . . . . . . . . 34

3 Running a Clock - A Software Revolution 442

3.1 The Experimental Sequence . . . . . . . . . . . . . . . . . . . . 42

3.1 .1 Synchronizing Two Clocks . . . . . . . . . . . . . . . . . . 44

3.2 The Slyfox Software Suite . . . . . . . . . . . . . . . . . 45

3.2.1 An Object-Oriented Programming Primer . . . . . . . . . . . . . 46

3.2.2 An Example: The Slyfox Frequency Synthesizer . . . . . . . . . . . . . . . 48 
3.2.3 Bringing it Together: The Slyfox Frequency Locker Program . . . . . . . . . 52

4 Where in the world is ${ }^{1} \mathrm{~S}_{0} \rightarrow{ }^{3} \mathrm{P}_{0} \quad 63$

4.1 Statistical Methods for Data Analysis . . . . . . . . . . . . . . . . 64

4.2 The Density Shift . . . . . . . . . . . . . . . . 65

4.3 The Zeeman Shifts . . . . . . . . . . . . . . . . . . . 69

4.3 .1 The First-Order Zeeman Shift . . . . . . . . . . . . . . . . . 69

4.3 .2 The Second-Order Zeeman Shift _ . . . . . . . . . . . . . . . . . 70

4.4 The AC Stark Shifts . . . . . . . . . . . . . . . . . 71

4.4 .1 The Scalar and Tensor Shifts . . . . . . . . . . . . . . . 71

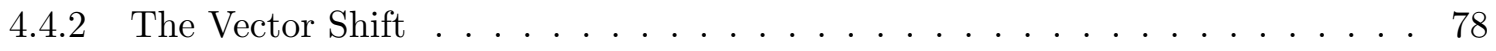

4.4.3 The Higher-Order Shifts . . . . . . . . . . . . . . . 78

4.5 The DC Stark Shift . . . . . . . . . . . . . . . . . . 79

4.5.1 Active Control . . . . . . . . . . . . . . . . 81

4.6 Miscellaneous Shifts . . . . . . . . . . . . . . . . . . . . 81

4.6.1 First and Second Order Doppler Shifts . . . . . . . . . . . . . . 82

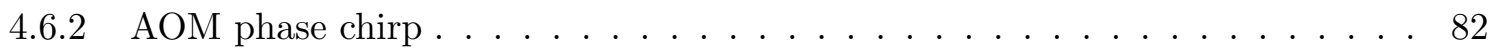

4.6 .3 Line Pulling . . . . . . . . . . . . . . . . . 84

4.6 .4 Probe Beam AC Stark . . . . . . . . . . . . . . . . 84

4.6.5 Background Gas Collisions . . . . . . . . . . . . . . 85

$5 \quad \mathrm{Sr}$ - The mini-COBE of Clocks $\quad \mathbf{8 6}$

5.1 Understanding the Shift . . . . . . . . . . . . . . . . 87

5.1 .1 Ray Tracing Model . . . . . . . . . . . . . . . . . . . 90

5.2 The Temperature Sensors . . . . . . . . . . . . . . . . . 93

5.3 Final SrII Error Budget . . . . . . . . . . . . . . . . . . 99

5.3 .1 SrI-SrII comparison . . . . . . . . . . . . . . . . . . 99 
6 The New Sr Quantum Gas Extravaganza Machine 103

6.1 Long Vacuum Lifetime . . . . . . . . . . . . . . . . . . . . 105

6.1 .1 The Pumpdown Procedure . . . . . . . . . . . . . . . . 110

6.2 Recessed Viewports for spectroscopic resolution . . . . . . . . . . . . . . . . 112

6.3 Optical Access . . . . . . . . . . . . . . . . . . . . 113

6.3 .1 Dipole-Dipole Interactions . . . . . . . . . . . . . . . . . . . . . 114

6.3.2 Spin-Orbit Physics in Alkaline Earth Atoms . . . . . . . . . . . . . 115

7 Summary \& Outlook

121

Bibliography

\section{Appendix}

A Single Purpose Fast Arduino Example

B Technical Drawings for SrIII Apparatus 


\section{Tables}

\section{Table}

4.1 Second Order Zeeman shift coefficients . . . . . . . . . . . . . . . . . . 70

5.1 BBR shift magnitudes for various atom species $\ldots \ldots \ldots$. . . . . . 87

5.2 SrII Effective Solid Angles . . . . . . . . . . . . . . . . . . . . . . . . 92

5.3 Diode temperature sensor corrections and uncertainties . . . . . . . . . . 96

5.4 SrII Systematic Uncertainties . . . . . . . . . . . . . . . . . . . . 100 


\section{Figures}

\section{Figure}

1.1 Stability comparison of the primary frequency standards and their competitors $\ldots \quad$. 6

1.2 Three leading systematics plaguing optical lattice clocks . . . . . . . . . . . 8

1.3 The ${ }^{87} \mathrm{Sr}$ Level Diagram . . . . . . . . . . . . . . . . . . . . . 11

2.1 Diagram of the $922 \mathrm{~nm}$ DBR laser . . . . . . . . . . . . . . . . 15

2.2 Diagram of Waveguide Doubler System _ . . . . . . . . . . . . . 17

2.3 Blue diode laser layout . . . . . . . . . . . . . . . . . . . . . . . . 19

2.4 Injection Lock Tracking Servos . . . . . . . . . . . . . . . . . . 20

2.5 CAD drawing of Horizontal Cavity . . . . . . . . . . . . . . . . . 22

2.6 ECDL lock to 813nm Buildup Cavity . . . . . . . . . . . . . . . 25

2.7 Diagram of injection locked Ti:Sapph lock to 813nm Buildup Cavity . . . . . . . . 27

2.8 Accelerometer traces of MOT coil shutoff $\ldots \ldots \ldots \ldots$

2.9 JILA Slow Loop Filter . . . . . . . . . . . . . . . . . . . . . . . . . 29

2.10 Diagram of the Auto Locker FSM . . . . . . . . . . . . . . . . . . 31

2.13 Optical Spectrum Analyzer measurements of Tapered Amplifier systems . . . . . . 35

$2.14 \mathrm{SrI}$ - SrII frequency with and without interference filters $\ldots \ldots \ldots$

2.15 SrI TA shift drifiting . . . . . . . . . . . . . . . 37

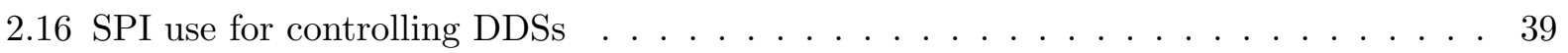

2.17 Screenshot of DDS control frontend . . . . . . . . . . . . . . . . . 41 
3.10 Screenshot of the Frequency Locker program . . . . . . . . . . . . . . . 56

4.1 Allan deviation of large lever arm density shift measurement $\ldots \ldots \ldots 8$

4.2 Field rezeroing using the atoms $\ldots \ldots \ldots \ldots \ldots$

4.3 Sigma transition minimization . . . . . . . . . . . . . . 75

4.4 AC Stark measurements . . . . . . . . . . . . . . . . 77

4.5 DC Stark shifts in SrII . . . . . . . . . . . . . . . . . 80

4.6 AOM Phase Chirp . . . . . . . . . . . . . . . . . . . . 83

5.1 Ray tracing model of the UHV vacuum chamber . . . . . . . . . . . . . 91

5.2 Diode temperature sensor picture . . . . . . . . . . . . . . 95

5.3 Zeeman slower heated window's effect on the temperature sensor . . . . . . . . 98

5.4 SrII - SrI Frequency Comparison _ . . . . . . . . . . . . . . . . 101

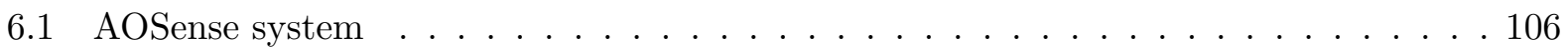

6.2 SrIII Main Chamber . . . . . . . . . . . . . . . . . 108

6.3 SrIII Recessed Viewports . . . . . . . . . . . . . . . . . 117

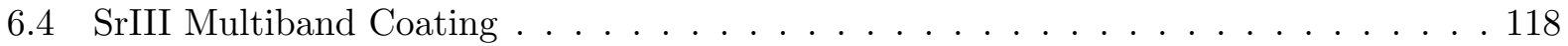

6.5 SrIII Broadband Coating . . . . . . . . . . . . . . . . . 119

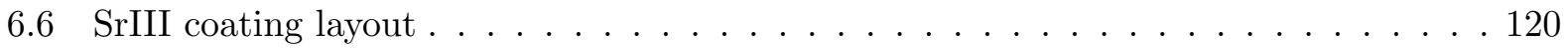

7.1 Red MOT absorption image of strontium atoms . . . . . . . . . . . . . . 124 


\section{Chapter 1}

\section{Introduction}

Measurement is a tricky business. In any quantitative experiment, a researcher tries to gain understanding of some unknown quantity by performing a differential measurement. Unbeknownst to most researchers, the differential aspect of their experiments is usually hidden under the moniker of whatever units they quote their result in. For most experiments, this is a trivial, usually forgotten point, Hertz are $\mathrm{Hz}$, Volts are V, and Henries are H. But for a select few experiments there exist, at this moment in time, measurements that have no absolute relationship to these units. This has not always been the case for optical lattice clocks, as even a few years ago frequency measurements, which were just at the cusp of performing better than the highest performing primary standards, were still possible via the use of optical frequency combs[1] .

Previous theses from the Ye group have referred to these standards coupled with optical frequency combs as rulers with equally spaced tick marks stretching from the radio-frequency $(\mathrm{RF})$ domain all the way to the optical domain [2]. However, it is the fractional frequency accuracy which is transferred with these ruler ticks, and not their absolute frequency uncertainty. In other words, looking at an absolute scale from the RF domain to the optical domain, the superbly narrow tick marks at low frequency become broader and fuzzier at higher frequencies.

While significant progress over the last 40 years has been made on primary frequency standards around the world [3], this technology is by every definition of the word mature. This is perhaps one of the most significant motivations for this work, the fact that new techniques and technologies offer a completely different approach to creating a stable frequency source. Essentially 
a top down method, where the standard is created in the optical domain and the stability and accuracy is transferred for use in the RF domain. The work presented in this thesis is merely an example of the ease of attaining an order of magnitude improvement to a new technology compared to squeezing out a factor of 2 from a mature technology. However, the rapid progress exhibited by the optical clock field comes with its own challenges. Not only do we demand state-of-the-art performance from both our atomic trapping apparatus and our optical local oscillator, but also by surpassing the accuracy of conventional frequency measurement techniques we were required to utilize differential measurement techniques, as no standards existed that were accurate enough for us to compare against. But why is it that Optical clocks have such an advantage over RF clocks? And what advantage does a neutral lattice clock have over other competing standards?

\section{$1.1 \quad$ Optical Lattice Clocks}

A primary standard must excel in three key characteristics - stability, accuracy, and realizability. The stability, or precision, of one's standard is the amount of noise inherent in taking a measurement with respect to that standard. This greatly affects the utility of the tool you are building. In fact, it has been repeatedly remarked that state-of-the art precision measurement experiments (eEDM, Atom Interferometry, Atomic Clocks, etc.) routinely attain accuracies near their quoted 1 day stabilities. Ultimate results will far exceed these numbers, but this is after years or decades of research $[4,5,6,7,8]$. Next, accuracy is the ability of one's measurement to be traceable back to an internationally agreed upon standard. In this case, because there is no agreed upon reference with comparable performance, we usually refer to the total systematic uncertainty of our clock. This measure is summed up in a list of all the external perturbations we know that shift our clock's frequency. The final piece of the puzzle is realizability, the ability to successfully disseminate this standard to anywhere one might require its accuracy - either via reproduction of standards or suitable transfer techniques - and to connect its accuracy to all measurements one might be interested in. With these three characteristics in mind let's examine ancient time keeping solutions, and the three leading contenders for current time keeping solutions. 
As humanity grew towards technological maturity, constructing time keeping devices and the definition of what time was became more accurate [9]. Older civilizations based time on astronomical calendars (including ours pre-1967), measuring the frequency of the rotation of the earth about the sun. Compared to the technology of the time, this oscillator was fairly stable. However, connecting this orbit to measurements on human time scales like seconds or hours was not a simple matter. Regardless of this complication, it is easy to argue why this makes a bad standard. Imagine trying to gauge the fractional uncertainty of measuring this frequency. First, in any measurement, there must be some error or limit to your precision with which you can take the measurement, $\delta f$. And second, one must simply normalize this measurement to get the fractional uncertainty, giving $\frac{\delta f}{f}$ or $\frac{1}{Q}$ where $\mathrm{Q}$ is the quality factor. With repeated measurements one might be able to measure more accurately, but with this figure of merit and an educated guess of $\delta f=1$ day for an astronomical calendar, it is no wonder that error in this method can accrue leading to the Catholic Church moving the calendar by 10 days in 1582 . Looking back it seems obvious that the construction of mechanical clocks with significantly higher $Q$ s due to their precise construction and much higher frequencies was the correct path to take. However, whether these clocks were mechanical pendula in nature, like in the industrial revolution, or electronic feedback from a quartz oscillator as is prevalent today, there is something unsatisfying with relying on man-made objects with their sensitivity to construction and external parameters to "keep the time" so to speak.

It was probably with this in mind that I. I. Rabi proposed probing energy levels in atoms or molecules as a source for a natural frequency (or time) scale. The primary standards we use today are a direct descendant of the first experiments built by Louis Essen and Jack Parry at the National Physical Laboratory in the UK and Norman Ramsey and Daniel Kleppner ${ }^{1}$ at Harvard $[10,11]$. To compare the primary Cs standards with optical clocks both ion and neutral, it will be useful to write down the simplest limit for frequency instability of uncorrelated atoms [12, 13],

$$
\sigma_{y}(\tau) \propto \frac{1}{Q \sqrt{\tau N}}
$$

\footnotetext{
${ }^{1}$ I sat behind N. Ramsey at a Colloquium once, and met D. Kleppner a few times as well.
} 
where $\mathrm{Q}$ is the quality factor of the transition you are probing, $\tau$ is the amount of averaging time related to the number of measurements one has made, and $N$ is the number of atoms. This limit known as the Quantum Projection Noise (QPN) limit for $N$ atoms can be reached when local oscillator noise no longer dominates (at some specific line Q given by the local oscillator noise). Cesium fountains work by probing the energy difference between the $6^{2} S_{\frac{1}{2}} \mathrm{~F}=3$ and $\mathrm{F}=4$ hyperfine ground states. By imparting a small upwards velocity to a cold gas of atoms, the atoms travel through a microwave cavity where they are excited, up into a shielded environment, and back through the same microwave cavity due to the influence of gravity [14, 15]. By double passing the same cavity many systematic biases associated with the excitation process are suppressed. So how do cesium fountains rate in each of the key characteristics? First, for stability, the transition being probed is in the microwave domain and the physical size of the fountain Fourier limits the interrogated linewidth. The high signal to noise and quiet local oscillators allow operation near the QPN Limit for $10^{4}$ or $10^{5}$ atoms reaching fractional frequency instabilities of $\sigma_{y}=4 \times 10^{-14} / \sqrt{\tau}$ [13]. However, running these clocks accurately with these atom numbers is quite difficult, and lower atom numbers and correspondingly lower stabilities are normally employed.

New results from a cryogenic Cs fountain clock at the National Institute of Standards and Technology are showcasing a new way forward for clock accuracy. Systematic error bars, known as Type B errors, for these systems of $1.1 \times 10^{-16}$ have been published, limited by technical details regarding the microwave cavities [8]. However, this does not include collisional shift uncertainties that are still large at $2.4 \times 10^{-16}$, which the authors categorize as statistical errorbars, known as Type A errorbars. Combining these results a total fractional frequency uncertainty of $4.4 \times$ $10^{-16}$ is reported. Finally, due to the wide use of RF, dissemination of this standard can be accomplished very easily with off-the-shelf components and contemporary satellite technology [16, 17]. Furthermore, the proliferation of Cs fountains in labs and metrology institutes around the world has proven their reproducibility.

To improve on this seemingly robust standard calls for a fundamental change in clock design. To significantly lower the instability of the clock it makes sense to dramatically increase the $Q$ of 
the clock by finding narrow transitions to probe with significantly higher frequencies of oscillation, $f$. Much like the move from an annual frequency to an electronic frequency, a new set of clocks have been constructed that interrogate optical frequency transitions rather than RF transitions. The first of these types of optical clocks to truly challenge the primary standards is the ion clock. The exquisite control of single quantum particles exhibited in these traps was recently acknowledged by the Nobel Prize in Physics in 2012 [18]. By confining ions in electrostatic (or effectively electrostatic traps) these ions can be sideband cooled to their motional and electronic ground states [19]. Utilizing an optical local oscillator with good short-term stability, an ion is excited into a superposition of ground and excited states, and a frequency discriminant to steer the laser onto atomic resonance at long time scales is created via Ramsey or Rabi spectroscopy [20]. As this technique for building a standard is our greatest competitor, due in part to its phenomenal performance, we will carefully analyze ion clocks in terms of our three figures of merit. Perhaps the biggest limitation of ion clocks is their large $\mathrm{QPN}$ as seen in equation 1.1. For a fixed line $Q$, due to say reaching the natural transition linewidth, stability is fixed for a single ion system. Regardless, due to the high $Q$ the instability can be as low as $\sigma_{y}=2.8 \times 10^{-15} / \sqrt{\tau}[21]$. In terms of accuracy, until the publication of the work documented in this thesis ion clocks held world record total uncertainties as low as $8.6 \times 10^{-18}[21]$, a stunning feat given their quoted stabilities. With the advent of the frequency comb, one could argue that the challenge of propagating this accurate standard to the $\mathrm{RF}$ is a solved problem, however ion clocks are somewhat burdened by the machinery surrounding their somewhat simple traps. Many of the laser transitions required to trap and cool the ions are deep within the UV making operation of these clocks difficult, expensive, and hard to miniaturize. However, as industries need shorter and shorter wavelength lasers and invest in R\&D for creating new classes of lasers, this might become less of an issue.

Turning to the final contender, the optical lattice clock (OLC), we will see that by trying to combine the many-atom, cold-gas nature of the Cs fountains with the precision afforded by optical clock techniques of ions might offer the best alternative. Much like a Cs fountain, an OLC starts by capturing atoms (usually Alkaline-Earth or Alkaline-Earth-like) inside a magneto-optical trap and 


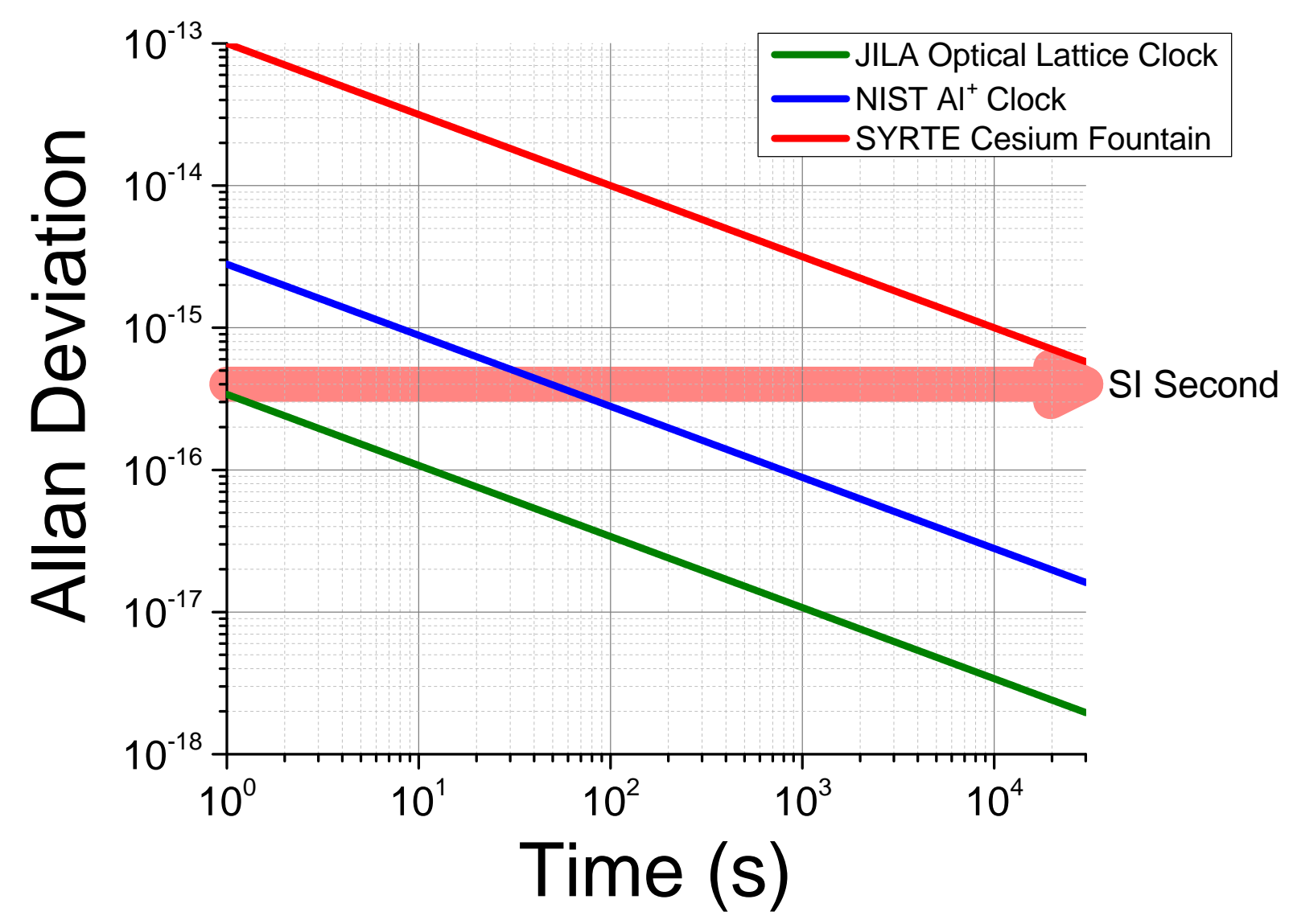

Figure 1.1: Stability comparison of the primary frequency standards and their competitors. The green line shows a fit to the demonstrated performance of the JILA SrII apparatus [22]. The blue line shows the performance of the Aluminum ion clock [21]. The solid red line shows the performance of a Cesium fountain run in high accuracy mode during a Rubidium vs Cesium comparison [23]. The light red arrow shows the current accuracy of many of the world's best Cesium fountains. 
then transferring them into an optical lattice created by a standing wave of light. While probing atoms with long wavelength radiation does not require the atoms to be trapped, simple Doppler shift formulas might fail as the atom traverses the wavefront of a laser. Furthermore, optically probing these atoms along the tightly-confining direction of the lattice allows separation of the motional and electronic states of the atoms, due to their trapping deep within the Lamb-Dicke and resolved sideband regimes inside the lattice [24]. The large number of trappable atoms, and high $Q$ of the clock transition, create wildly small and presently unattainable instabilities via equation 1.1. Instead, the stability of these clocks is limited by the state-of-the-art laser local oscillators used to interrogate them [25]. This technology dependence creates a constant pressure to innovate new advances in laser frequency control techniques as any progress in the area of frequency stabilization can immediately be put to use to further improve the performance of the atomic clock. Accuracy in these systems had until recently lagged behind ion clocks. The added difficulties of controlling atom-atom interactions, differential light shifts due to the trapping light, and the relatively higher sensitivity to blackbody radiation shifts on the clock all had to be addressed.

This thesis documents the JILA Sr clock, the first optical lattice clock that bested all other atomic clocks with a low total systematic uncertainty of $6.4 \times 10^{-18}$ [22]. A testament to the realizability of this system is the increasing number of OLC experiments popping up around the world, especially with the Strontium isotope which exhibits laser cooling and trapping wavelengths all accessible via diode lasers $[22,26,27,28,29,30]$. Perhaps unsurprisingly, the benefits imparted by using ever more stable laser local oscillators to interrogate the atoms make the state-of-the-art versions of these clocks very sensitive to thermal noise of both the cavity spacer and mirror coatings, and presently limits miniaturization of these systems. Future technologies might make these clocks even more attractive [31].

Examining Fig. 1.1 it is easy to see why optical lattice clocks are considered so promising. However, moving from a carefully controlled system with one particle to that of many particles was rife with challenges. As more strontium experiments have been built, an enormous, international body of work has grown up to defeat the obstacles listed below. First and foremost of these 
challenges was the intrinsic deformation of the valence electron cloud of the neutral atom to actually trap it for clock interrogation. The so-called "magic" wavelength trap is employed to counteract these effects, in that the differential light shift between the ground state, ${ }^{1} \mathrm{~S}_{0}$, and the excited clock state, ${ }^{3} \mathrm{P}_{0}$, caused by the trapping light is nearly zero [32]. Higher order corrections might still come into play, and this shift will need to be measured as precise knowledge about the zerocrossing point is only ever known to the precision with which you measure it. Atom interactions were thought to be nonexistent in the original proposals, as spin-polarized ultracold fermions should have severely suppressed s-wave collisions and low p-wave collision rates. But as is often the case, at the frontiers of precision measurement, even tiny effects can play a leading role in one's final uncertainty [33]. Finally, the largest limitation to accuracy came from the influence of broadband blackbody radiation (BBR) impinging upon the atoms causing an uncontrolled AC stark shift. A topic that will be greatly expounded upon in subsequent chapters.
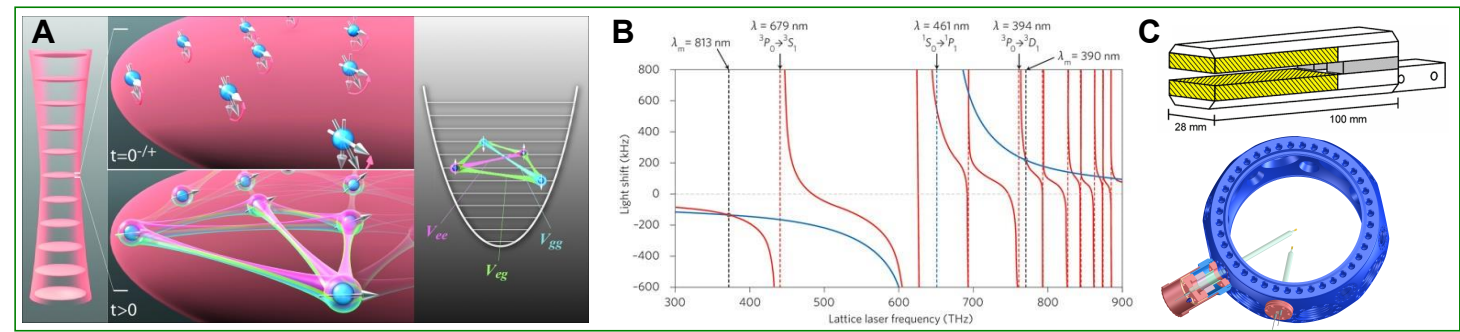

Figure 1.2: The three leading systematics plaguing optical lattice clocks and how they have been conquered. A) Exhaustive studies of the collision shifts were performed, not just probing their shift of the clock transition, but their effect on atom shot noise [34]. This image is taken from Ref. [34] B) The AC stark effect from operating the clock near the magic wavelength has been studied around the world $[22,26,35]$. This image summarizing AC stark shifts on the clock transition in the visible and NIR spectrum is taken from Ref. [36]. C) An image from Ref. [37] depicts the DC polarizability measurement that enabled our use of the temperature probes as a means for inferring the BBR stark shift. Below the image of the DC electrodes is a CAD file depicting the mounting of our BBR sensors inside the JILA SrII vacuum chamber.

So while the path forward for state-of-the-art clocks seems clear, the inqusitive reader might wonder what all the fuss is about. While a more mundane answer might center around the advances in creating UV sources of light and the accuracy of performing spectroscopy with these sources, 
a host of other more exotic uses for these clocks might exist. In fact, comparing optical clocks made with the same species has already been used to provide more stringent restrictions on the coupling of fundamental constants to weak gravitational forces [38], and in the future measuring frequency ratios of highly accurate optical clocks will put stringent limitations on the drift of the fine structure constant with time $[38,39]$. As stable long term oscillators, optical lattice clocks

offer a path forward for sub-mm wave very long baseline interferometry (VLBI) [40]. In VLBI one creates a phase coherent array of antenna that can be used to generate a more detailed picture of the galactic source one is imaging. The distance between disjoint VLBI stations allows one to create planetary scale effective antenna sizes. Current limitations with Hydrogen masers keep these techniques well in the RF, whereas a dramatic increase in stability could open the door for future experiments. The sensitivity of these clocks to gravitational redshifts might also allow these clocks to be used as tools for relativistic geodesy to map out the earth's gravitational field [41]. Not only might these clocks be used to study such fundamental physics like variation of fundamental constants [42], help in the search for dark matter [43], or the coupling of gravity to other forces in nature [38] but also they will continue to allow us to probe complicated artificial quantum manybody systems $[34,44]$. Whatever path is taken to create these optical lattice clocks, their future seems bright.

\subsubsection{The JILA ${ }^{87}$ Sr Lattice Clock}

The results presented in this thesis are based on the second generation JILA Sr lattice clock, referred to throughout this thesis as SrII. The differences between this new apparatus and SrI were made to fix many of the issues associated with creating a highly accurate lattice clock. But how does one create a strontium lattice clock? A brief summary is presented below.

An ultracold gas experiment traps incredibly low density gases, at $\mu \mathrm{K}$ temperatures, in extremely weak traps. This requires an incredibly low background pressure inside the trapping volume. In the case of strontium cold-atom-machines this poses quite a challenge as the vapor pressure of strontium, a solid at room temperature, is very low. Heating solid chunks of strontium 
to $575 \mathrm{~K}$ in an effusive oven, the hot vapor can be collimated towards the main trapping chamber. Heating up the steel of the vacuum chamber creates a large gas load for ultrahigh vacuum pumps in the region of the oven, so differential pumping between the oven region and the main chamber is a must. The hot vapor streams down the apparatus, past a mechanical shutter, through a differential pump hole, and enters a zeeman slower. Strontium has a slightly more complicated level structure compared to an alkali atom as can be seen in Fig. 1.3. However, a mostly closed dipole-allowed transition exists between ${ }^{1} \mathrm{~S}_{0}$ and ${ }^{1} \mathrm{P}_{1}$. This fairly broad transition is used first to cool the atoms' forward-motion in the Zeeman Slower and finally to catch the atoms in the main chamber with a Magneto-Optical Trap (MOT). This "blue" MOT traps the atoms at a few mK. The intercombination transition between ${ }^{1} \mathrm{~S}_{0}$ and ${ }^{3} \mathrm{P}_{1}$ is $7.4 \mathrm{kHz}$ broad and allows the atoms to be cooled down to less than $1 \mu \mathrm{K}$ in just a few hundred milliseconds [45]. It is then that the atoms are loaded into the magic wavelength trap created with a laser at $813 \mathrm{~nm}$ forming a standing wave inside a buildup cavity creating an array of two dimensional isolated atom traps. In this final trap, the clock transition of the atoms can be probed in the tightly confining direction as a laser tuned precisely to the transition near $698 \mathrm{~nm}$ can coherently Rabi flop the atoms from ${ }^{1} \mathrm{~S}_{0}$ to ${ }^{3} \mathrm{P}_{0}$. The ensemble average of the coherent electronic superposition across the cloud can be read out by first destructively fluorescing atoms in the ground state and then measuring the remaining atoms that were shelved in the excited state. This entire experimental sequence can be repeated at a duty cycle of approximately $1 \mathrm{~Hz}$, and the optical local oscillator can be locked to the atoms or used as a stable reference to measure various frequency shifts to the clock transition when external parameters are varied in a controlled fashion. 


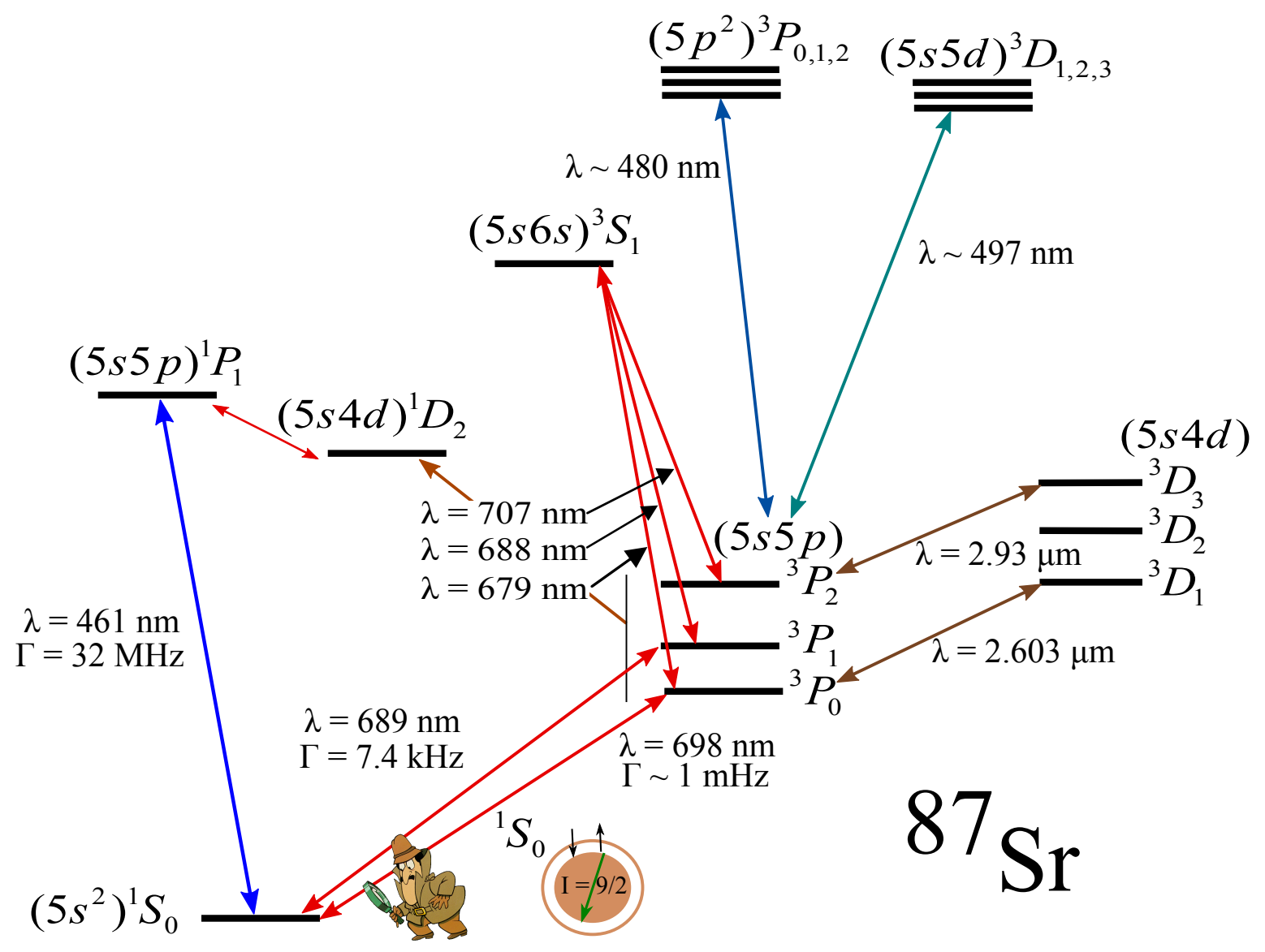

Figure 1.3: The ${ }^{87}$ Sr Level Diagram. Based off of a Figure from Martin Boyd's thesis [46]. 


\section{Chapter 2}

\section{Building a Clock}

When I first arrived as a bright-eyed, happy-go-lucky graduate student the first iteration of many of the major systems on SrII was complete. At the time the majority of our focus was to explore the optical feshbach resonance technique in ${ }^{88} \mathrm{Sr}$. Imaging and final trapping were not quite there, but we had cold atoms and the sky seemed the limit! Unfortunately, it turned out the vacuum lifetime $(\sim 1 \mathrm{~s})$ was the actual limit, and once we were done with the feshbach studies in bosonic strontium, the decision was made to pursue the dream for a far more accurate clock. To

accomplish the feat of switching to the fermionic ${ }^{87} \mathrm{Sr}$ isotope and creating a really robust system that could operate and take data for hours at a time with minimal human intervention a lot of system components needed to be revamped or upgraded. In addition to the system components I will discuss below there were many components like the MOT coil switching electronics, the bias field electronics, the atom detection systems, and the red laser systems - to name a few - that required significant overhauls that are not documented here.

\subsection{The Blue Laser System}

Until recently, creating a good $461 \mathrm{~nm}$ laser system was not something one could take for granted. The ${ }^{1} \mathrm{~S}_{0}$ to ${ }^{1} \mathrm{P}_{1}$ transition is incredibly important for all laser cooling and trapping of strontium. However, due to its relatively high frequency and large linewidth of $32 \mathrm{MHz}$, compared to the D1 or D2 lines of alkali elements, its saturation intensity, $\mathrm{I}_{\text {sat }}$, is a painfully high $43 \mathrm{~mW} / \mathrm{cm}^{2}[47]$. While high intensities are not necessary for optimal first-stage MOT densities, conventional Zeeman 
slower design constraints, and fluorescence detection call for beams approaching the saturation intensity. Therefore powers approaching or exceeding $50 \mathrm{~mW}$ delivered to the atoms at a variety of detunings are not unheard of requirements for successful fermionic strontium experiments. A variety of early approaches to creating this light involved either dye lasers or second harmonic generation of titanium-sapphire (Ti:Sapph) lasers [45].

\subsubsection{The Old Blue laser System}

The SrI system uses a 922nm external cavity diode laser (ECDL) that seeds a tapered amplifier (TA) that is then coupled into two doubling cavities. These standing-wave second harmonic generation cavities use $\mathrm{KNbO}_{3}$ with the back flat facet of the potassium niobate crystals having been HR coated for both the IR light and the blue light. The phase matching temperature of these crystals is a toasty $150{ }^{\circ} \mathrm{C}$, however their doubling efficiency exceeded $50 \%$. This system had been in continuous use for a few years before the first graduate student on SrII, Sebastian Blatt, had to decide how to build SrII's blue laser system. Operating temperatures in these systems were within tens of degrees to the crystals repoling temperature, not only making the use of these cavities cumbersome but also nerve racking. Examining the literature at the time the reports in Ref. [48] of $75 \%$ SHG conversion efficiency were too good to ignore. The system described in Ref. [48] utilized a periodically-poled potasium titanyl phosphate (PPKTP) critically phase-matched crystal inside a ring resonator and so the decision was made to try a PPKTP system. However, due to the experience in lab working with standing wave cavities, the PPKTP was built in a standing wave configuration. Unfortunately, with subsequent years of experience working with this system, it became clear that color center formation severely degraded the performance of this system throughout the day, making long experimental runs quite trying. Rejuvenation of the crystals was possible by baking them overnight, but the constant fluctuations of $>60$ degrees were an alignment nightmare. Therefore, the SrII group was always on the lookout for ways to improve the reliability of all components in the $461 \mathrm{~nm}$ setup.

The first major upgrade to the system was an upgrade to the IR laser diode. Over the 
course of a few years, we noticed that the Ridge Waveguide lasers that we used from Eagleyard would work for a few months, and would subsequently become more and more finnicky as time went on, probably due to decay of the AR coating on the diode's front facet. Luckily, we found that Photodigm, a fairly new company, was trying to expand their offering of distributed bragg reflector (DBR) lasers. A DBR laser is in some sense a miniaturized ECDL, with the feedback being provided by a distributed bragg reflector built on the same substrate as the laser gain medium. This should not be confused with a distributed feedback (DFB) laser where the active gain medium of the laser is modulated to support lasing on only certain wavelengths. The DBR laser we received from Photodigm throughout initial testing and day to day use over two years was incredibly stable and very compact. Fig. 2.1 shows the very nice package the laser came in, including both a TEC and thermistor for good thermal control of the laser diode. Care must be taken with these lasers, as they are incredibly sensitive to feedback, even allowing for dramatic linewidth reduction given the correct optical feedback [49]. In fact, when installing this laser into the 461nm SHG system, additional optical isolation was necessary to allow stable operation. Regardless, I would have to say that this DBR laser was the best behaved laser I have ever worked with.

\subsubsection{New Additions to the Blue team}

January 2011 started with a complete blue system realignment. We were switching from the more abundant bosonic isotope of strontium to the less abundant fermionic isotope of strontium. We needed every blue photon we could muster, and the output power of both SrII blue doublers had been on a very slow downwards trajectory for almost two years. Three tasks were clear though, more power in the MOT, more Zeeman Slower power, and implement transverse cooling before the atoms entered the Zeeman Slower area.

The blue MOT light was delivered to the atoms via a Schäfter+Kirchoff 1x3 fiber splitter. This component had been in use for many years, and a careful measurement of the input-ouput powers of this splitter revealed something quite surprising. Almost $80 \%$ of our blue light was being lost in the fiber couplings into and out of this device. In fact, even at its best, its specified operation 
A

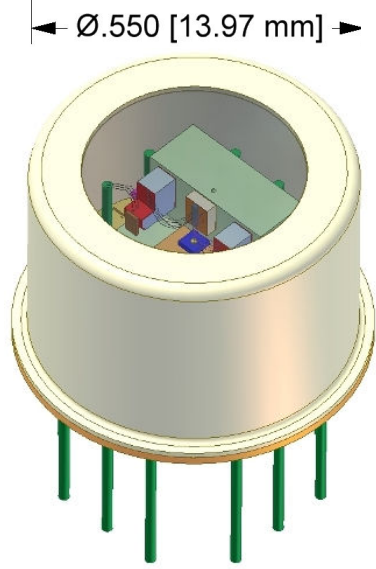

B

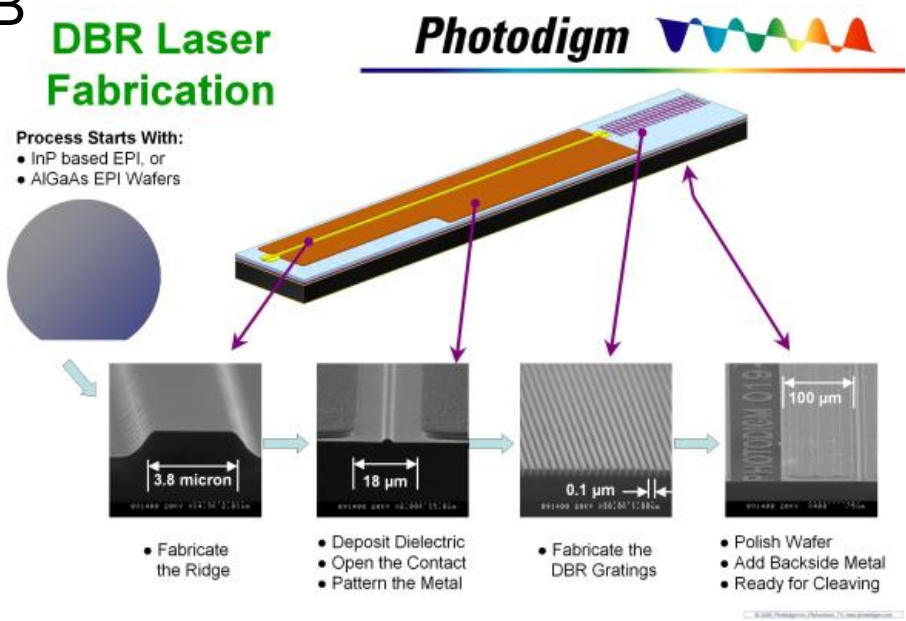

Figure 2.1: A) A diagram of the 922nm DBR laser package including a thermistor and TEC for temperature control. B) Model of a DBR laser showing both the gain medium and the grating feedback. Additional SEM images show the construction of a DBR laser at Photodigm. Both Images are taken from promotional material from the Photodigm website. 
called for at least $40 \%$ of the light to be lost. After contacting the company, purchasing their specialty tools, and aligning the system from scratch, we were back to operation above specifications. However, a close eye was kept on the device, and within 6 months a clear degradation of fiber couplings was seen again. Another solution was needed. Jason Williams, the postdoc on SrII, had previously had a good experience using evanescent wave beamsplitters. We contacted Evanescent Optics Inc. (formerly Canadian Instrumentation \& Research Ltd) and they offered us fiber splitters at a fraction of the cost. These have now been in use for years as well, with no noticeable change in performance. The MOT problems were solved, and a subsequent realignment of the second doubling cavity brought the Zeeman Slower powers in line as well. ${ }^{1}$

However, we were still no closer to garnering enough blue power for transverse cooling. It should be noted that due to the long distance between the oven and the main chamber, our overall atom flux was lower than the upgraded SrI system. In the end we utilized a single-pass periodicallypoled lithium niobate (PPLN) waveguide doubler to produce about 60-70 mW of $461 \mathrm{~nm}$ light. Produced by NTT, these waveguide modules can be customized for SHG, SFG, or DFG. These doublers combine the critical phase matching and high nonlinearity of other periodically poled materials built into a waveguide structure to keep the input light at a high intensity throughout the nonlinear medium. The relative simplicity of using this system can be seen in Fig. 2.2. With this blue light we were able to construct both a multi-bounce vertical and horizontal transverse cooling setup that easily increased our final atom number by a factor of 3 .

\subsubsection{The Blue Diode System}

Regardless of the ever-improving performance of SrII system, it was still a rather complicated system for something so basic and intrinsically necessary for a strontium clock. Hopes of portable strontium lattice clocks hinge on the ease of use of the strontium atom. So it was perhaps with a collective sigh of relief that SrII acquiesced to testing out some new blue diode laser sources for New Focus/Newport. While it remains unclear exactly the source of the AR coated diodes used in New

\footnotetext{
${ }^{1} 922 \mathrm{~nm}$ powers were measured to be $\sim 1.1 \mathrm{~W}$, in a poor spatial mode, creating $\sim 120 \mathrm{~mW}$ of blue light.
} 

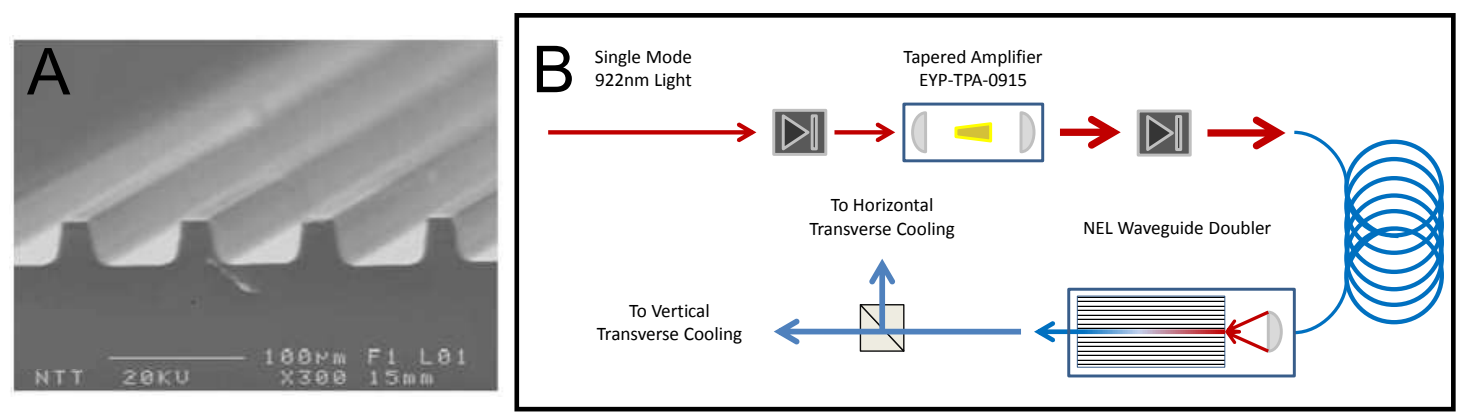

Figure 2.2: Diagrams of the waveguide doubler system used for transverse cooling. A) SEM image of the waveguide structure imprinted into periodically poled $\mathrm{Zn}: \mathrm{LiNbO}_{3}$. Image is from Ref. [50]. B) Schematic diagram of the system used for creating $461 \mathrm{~nm}$ light for transverse cooling of the atoms. Not all components are pictured for figure clarity. 
Focus's laser, the results speak for themselves. The $461 \mathrm{~nm}$ module started off slightly multimode, but with an impressive $45 \mathrm{~mW}$ of output power. After temperature tuning the diode more carefully it was back to single mode. Throughout the last 18 months the power has degraded slightly, but has seemed to level off at about $35 \mathrm{~mW}$. While at first temperature and careful PZT and current tuning were a necessity, the blue module has seemed to hit its stride and needs much less tuning than it even did 10 months ago. Luckily, at the same time that New Focus started building these 461nm modules, Nichia started offering 460nm blue diodes. These blue diodes while advertised as single mode diodes that can put out $120 \mathrm{~mW}$ of power, aren't quite single mode at all at powers above a few $\mathrm{mW}$. But, they can be used like slave lasers or slave amplifiers. Injection locking of these blue diodes is possible with powers as low as $500 \mu \mathrm{W}$ although stable operation is better at $1 \mathrm{~mW}$ injection power. In Fig. 2.3 one can see the new blue laser setup.

Two important design considerations were kept to ensure successful system operation. First, the master laser is locked directly to the strontium discharge cell. This allows for the master to remain locked for however long is possible given the stability of the New Focus Vortex laser, usually an entire day. Second, the vast majority of frequency steering is done before power amplification to minimize wasting precious blue photons. In periods of quiet operation all lasers, both slave and master, remain locked for hours at a time. However, if temperatures are changing quickly in the lab injection lock can be lost. This has spurred an effort into active injection tracking servos that is still being worked on.

\subsubsection{Injection Locking Servos}

Long term stability of the blue lasers is very important in the operation of a clock. Datasets can run anywhere from 10 minutes to 10 hours, so having any system component not perform at $100 \%$ uptime is unacceptable. Spurred by some curious effects we observed in the photodiode signal we started looking into active injection locking servos. Before much research had been done into the subject, Igor Teper and Tom Loftus from AOsense pointed out three very useful papers on the matter of optimum current set point servos in various diode laser configurations $[51,52,53]$. 


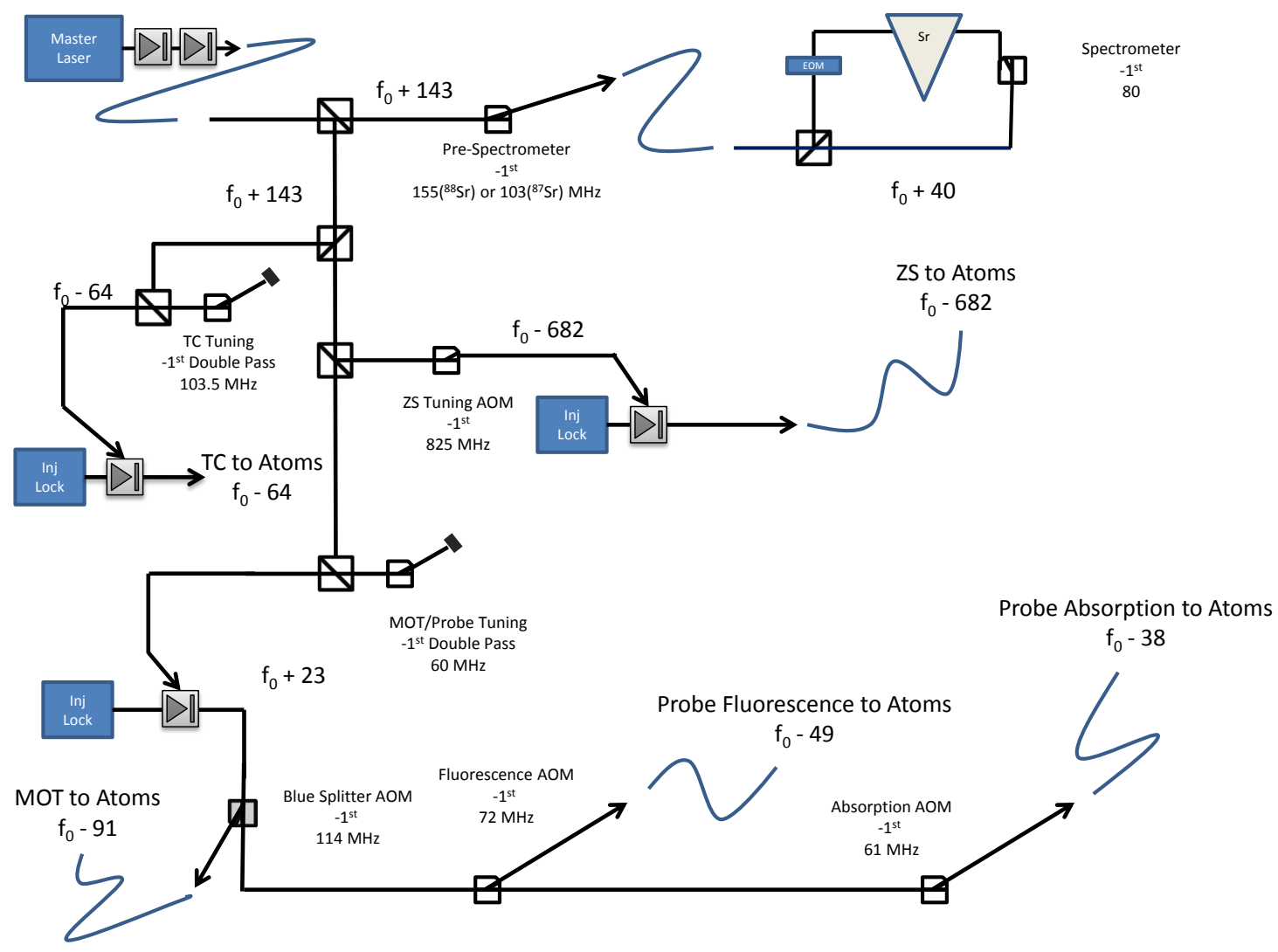

Figure 2.3: Blue diode laser layout showing frequencies and placements of all AOMs. All numbers are in units of $\mathrm{MHz}$, and $\mathrm{f}_{0}$ represents the $\mathrm{Sr}^{1} S_{0^{-}}{ }^{1} P_{1}$ transition frequency. All injections locks are performed by sending in light through an isolator's rejection port. Not all components are pictured for figure clarity. 
For the purpose of explaining how these servos derive their error signal, let us take the example of an uncoated diode placed inside an external cavity diode laser setup. In this system, there are two competing modes trying to saturate the laser gain medium, the laser cavity mode associated with the laser's front and back facets, and the external cavity mode between the laser's back facet and the feedback grating. When these two cavity modes come close to resonance with each other, the feedback resonance starts to saturate the laser diode, and the system will exhibit single mode operation. Bringing these two cavity modes close together can involve walking many parameters that effect the gain curve of the laser and the length of both cavities. Focusing on the angle of the grating and current setpoint of the laser diode, an optimum value of the lasing current must exist where small variations in the resonance position of the external cavity will not push the laser away from single mode operation.

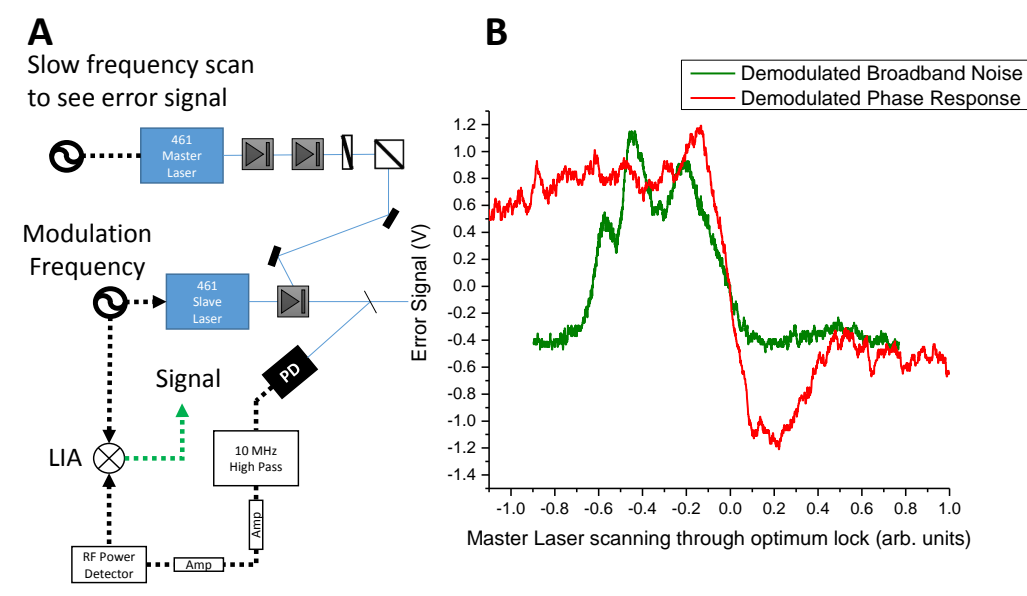

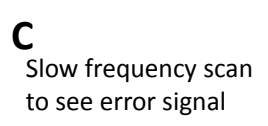

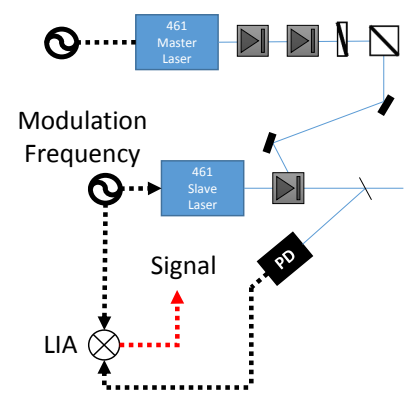

Figure 2.4: A) Diagram showing the procedure of measuring the broadband noise response of a slave diode to determine optimum slave laser locking current using a Lock-In Amplifier (LIA). B) The signals produced from either the broadband (Green) or phase measurement (Red) of the slave diode. The $\mathrm{Y}$ axis is in volts, however, an arbitrary offset has been subtracted. The position of the waveforms at $\mathrm{x}=0$ is where one would lock diode current. Neither seems to occur at the steepest discriminant in the signal, but a feedback signal seems possible for both methods. C) Diagram showing the procedure for measuring the phase response of the slave diode.

Finding the optimum setpoint is not difficult, but if parameters change that are out of the experimentalists control (e.g. temperature or humidity) or even if they are in your control like 
scanning the frequency of the laser over a large range, it would be nice for the current to track to the optimum setpoint. In Fig. 2.4 the signals obtained and the layout of two different methods for obtaining a locking signal are shown. In the first method, the laser's current is modulated at a frequency $f_{m}$, and its intensity noise is measured with a bandwidth above $f_{m}$. The photodiode current signal is then demodulated at $f_{m}$, and the voltage output from a lock-in amplifier gives the phase relationship between the induced amplitude noise and the current driving signal. The phase relationship depends on the transfer function of the laser diode, and not only is it stable, but it smoothly changes with the current of the laser near the optimal setting $[51,53]$. In essence, one is just measuring the frequency modulation to amplitude modulation transfer function of the laser at one specific frequency, $f_{m}$. While this method produced a reasonable error signal to lock to and was less complicated, I experimented with the method in Ref. [52] and found it to be more robust. In this method we module the laser current at $f_{m}$ but then look at the broadband amplitude noise at significantly higher frequencies than $f_{m}$, above $10 \mathrm{MHz}$. To gain information about the noise, a Minicircuits RF power detector (ZX47-40+) was used. This signal is then demodulated at $f_{m}$ giving essentially a noise measurement for a sweeping setpoint current. When the laser is mostly single mode, the amplitude of the high frequency noise goes down, measuring this signal in a phase sensitive manner produces an odd signal that one can feedback to the laser current. Here, one is measuring the frequency modulation to amplitude modulation transfer function of the laser at all frequencies from $10 \mathrm{MHz}$ to the detection bandwidth of our photodiode (180 MHz), and integrating that response with an $\mathrm{RF}$ power detector to arrive at one single voltage for each current setpoint. While these tests were performed with a commercial lock-in amplifier, which would be a needless expense for every injection lock laser, our youngest member on the strontium team is building a homemade board suitable for creating these error signals.

\subsection{The Magic Wavelength Buildup Cavity}

Careful comparison of the initial reports of cold collisional shifts in fermionic strontium clocks [33] and the lack of similar shifts in other clocks, that were still reporting lower measurement 

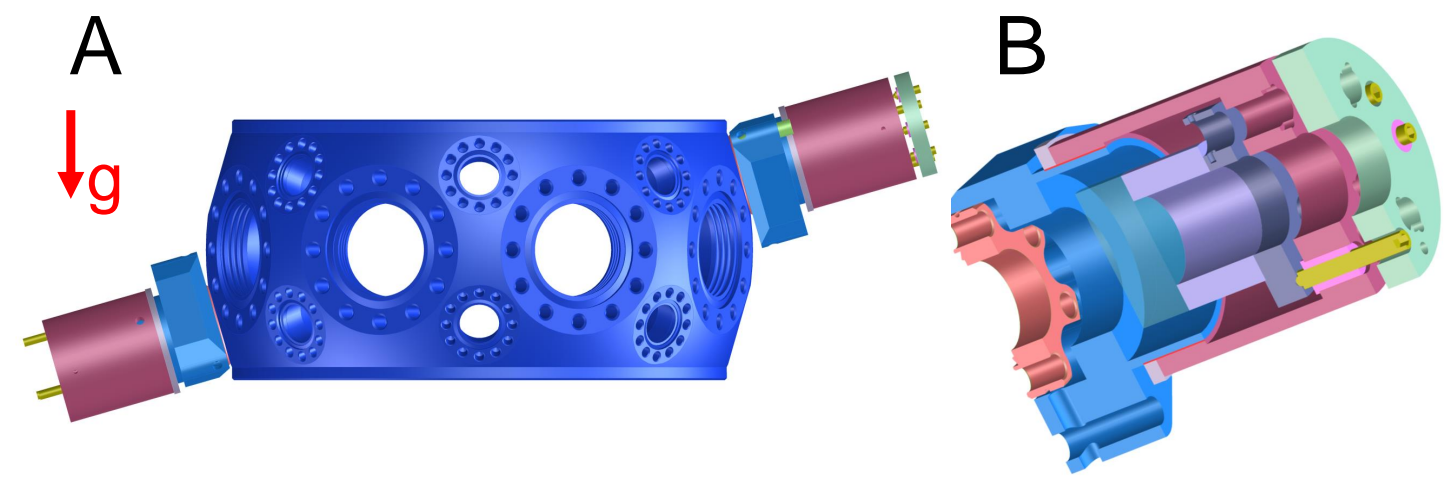

Figure 2.5: CAD drawings of the horizontal cavity mounts. A) This CAD drawing shows the placement of the two horozontal mounts on the Kimball Physics chamber, mounted on two opposing CF133 viewports. B) A cutaway of the internal structure of the mount that holds the fixed mirror for the cavity. It is important to note that this mount can both steer the cavity mirror and has an additional steerable plate for use in retroreflecting a beam for fiber phase noise cancellation.

precision, was for almost a year a point of contention in the optical lattice clock community. However, key design differences between the JILA and SYRTE atomic clocks were discovered. While the original JILA clock, SrI, was formed by trapping in the tight focus of a 30 micron waist sized beam overlapped with the dense red MOT, the SYRTE clock was a drain loaded clock held in a cavity enhanced lattice eschewing the cold dense red MOT and having very poor atom transfer between the blue MOT and the lattice. The resulting differences in densities completely explained the discrepancies [54]. It was with this idea about the fundamental connection between cold collisional shifts and density (rather than atom number) that we decided to build a nearly horizontal build-up cavity on SrII. ${ }^{2}$ Not only would this setup give us better spatial overlap with the red MOT, allowing us to transfer a larger number of atoms into the magic wavelength 1D lattice, but also they would be trapped at a lower density and would induce a lower density shift.

The magic wavelength for the ${ }^{1} \mathrm{~S}_{0}$ to ${ }^{3} \mathrm{P}_{0}$ transition in ${ }^{87} \mathrm{Sr}$ is at approximately $813 \mathrm{~nm}$ (See Fig. 1.2). Luckily this is a wavelength that can be reached with laser diodes and is also accessible with a cw-Ti:Sapph laser. Here I will describe the use of both versions of an 813nm system suitable for atom trapping. The build up cavity is external from the vacuum system, which limits the

\footnotetext{
${ }^{2}$ The lattice axis is $16^{\circ}$ above the horizontal, lifting the lattice site degeneracy
} 
intracavity power due to the losses in the viewports. However, having a cavity inside the vacuum is not without its own challenges, especially given the sensitive nature of our spectroscopy. In fact, placing dielectric surfaces near the atoms caused significant problems for the SYRTE group. Their mirrors themselves became charged inducing DC stark shifts on their clock transition on the order of $10^{-13}[55]$. In addition to this concern we have found the ability to move the cavity mode to the chamber's magnetic field zero, rather than it's geometric center, to be very useful. For the majority of the work presented in this thesis, the cavity mirrors in our system are located on two opposing CF133 flanges as depicted on the CAD model in Fig. 2.5. The mounts are adjustable, like a kinematic mirror mount, and allow for small movement of the cavity mode in the system. They are not identical mounts, as one is built to hold a cavity mirror that has been glued onto a stack ring piezo. These piezos were bought from Physik Instrumente and allow beams to enter at the center of the cavity mirrors.

The cavity supports a TEM00 mode with a beam radius of $160 \mu \mathrm{m}$ formed by two mirrors with $20 \mathrm{~cm}$ radius of curvature. The mirrors are coated for $98 \%$ reflectivity at $813 \mathrm{~nm}$ and $0.04 \%$ reflectivity at $698 \mathrm{~nm}$ to allow for very little interference, or superlattice effects, in the clock excitation over the atom cloud. Losses through the viewports were measured with an extra viewport from the same coating run. A spectrophotometer showed $97.8 \%$ transmission through a single AR coated viewport. The finesse can be calculated in two ways, either with the FSR and the FWHM of the cavity or with knowledge of the intracavity losses. Both methods agree, showing a finesse of 70. As mirrors and coatings were ordered at the same time as the SrII vacuum chamber, and were not ordered with a variety of reflectivities the cavity is not impedance matched. This results in a power build-up factor of about 18 . So for $330 \mathrm{~mW}$ actually coupled into the cavity, SrII achieves a circulating power of 6 Watts $[56,57]$. Impedance matching could in fact bring this factor closer to 37. For that matter better mirrors, that are also impedance matched, could still achieve an overall enhancement factor of 44. This is more than good enough for our purposes, and suggests that the trouble of building an in vacuum cavity is not worth it if power enhancement is your only goal.

Many options are available to lock the $813 \mathrm{~nm}$ light to the buildup cavity. Unfortunately 
the large size of 1 inch mirror glued onto the equally large ring piezo caused fairly low frequency resonances in the actuation of the piezo. The locking bandwidth of the piezo is purporsefully kept low to avoid ringing of the cavity. In the case of the diode system, the current of the diode allows for a large bandwidth lock to a cavity. In Fig. 2.6 one can see how both a fast feedback path to the diode laser current was created, and a slow feedback path to the cavity piezo was included. A Pound-Drever-Hall (PDH) lock was constructed for actually keeping the laser on resonance with the cavity. Excellent writeups abound explaining the PDH locking technique [58]. However, my favorite and most useful reference for this has been a NIST Time and Frequency writeup that includes descriptions of the electronic locking circuits involved [59].

Because the atoms react poorly to intensity noise, especially to harmonics of their trap frequencies, it's very important that the lock to the cavity is high bandwidth [60]. Any relative frequency noise or excursion by the laser is in fact transformed into amplitude noise inside the cavity, which can parametrically heat the atoms. Since we are interested in keeping the atoms from being heated and holding them in the trap unperturbed, we sample the transmitted light from the cavity and add intensity stabilization feedback to further supress any residual amplitude noise. While SrII used the setup depicted in Fig. 2.6 for many months, one or two oddities of the design made us rethink our approach in subsequent apparatus upgrades. For example, the large distance between the EOM which placed sidebands on the laser and the path length of the reflected beam for the PDH lock were significantly longer than they needed to be. Unfortunately, this turned out to cause a lot of instability in the system, as residual amplitude modulation (RAM) picked up from stray reflections throughout the path caused fairly large offset swings.

While this system could be locked to the cavity with a high-bandwidth actuator, the injection locked Ti:sapph did not naturally have such an actuator. While it is possible with the use of a nonlinear transmission line and an EOM to lock light to a cavity, the SrII group decided to try the more mundane approach of double-passing an acousto-optic modulator (AOM). In Fig. 2.7 one can see the added complexity of using an injection locked Ti:Sapph. Unfortunately, the locking bandwidth of this setup to the cavity is only a few hundred kilohertz and the dynamic range is set 

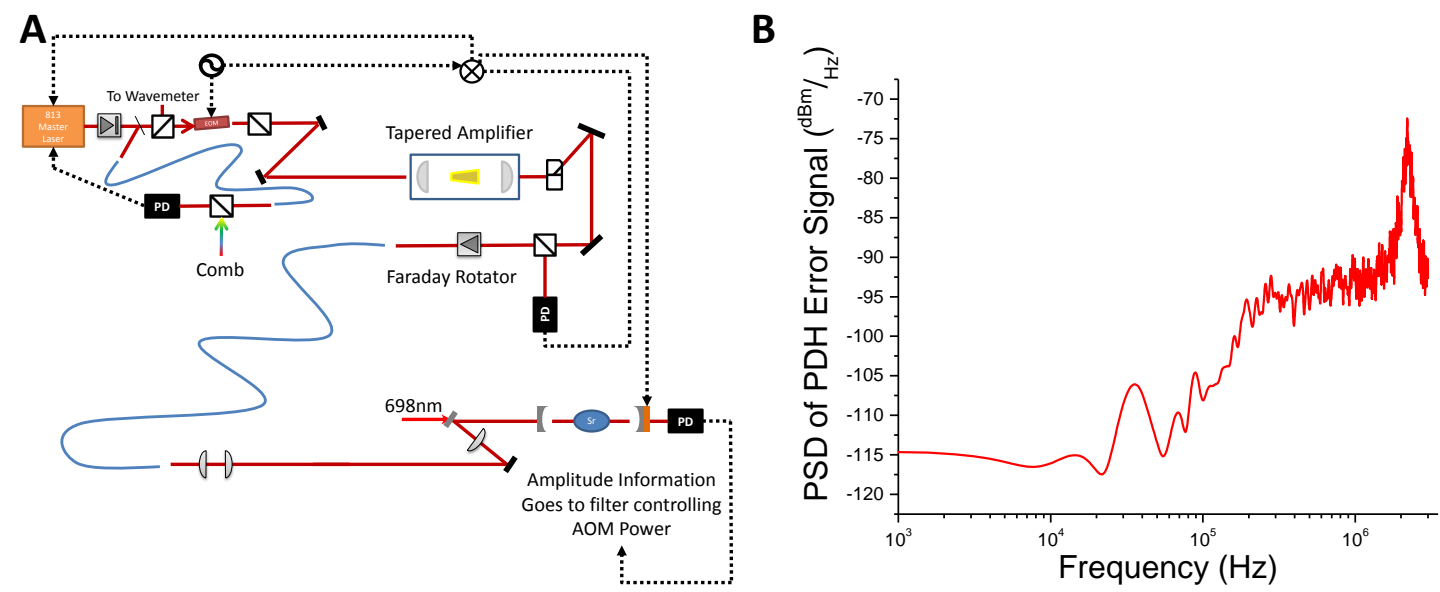

Figure 2.6: ECDL lock to 813nm Buildup Cavity. A) Diagram of the 813nm ECDL-based system and its lock to both the frequency comb and the buildup cavity. Not all components are shown in diagram. B) Power spectral density of the in loop locked error signal for the lock to the buildup cavity. The high bandwidth is achieved due to the fast actuation of the current of the diode and the large dynamic range of the system, making this very robust against cavity vibrations. 
by the tuning bandwidth of the AOM and how much steering is caused by changing the frequency. In fact, this system deals with perturbations to the buildup cavity very poorly.

Jolts or jerks to the system would routinely cause the system to immediately come out of lock. When first set up, the system could not survive the Blue MOT $(60 \mathrm{G} / \mathrm{cm})$ to red MOT (5 $\mathrm{G} / \mathrm{cm}$ ) MOT coil switch. This fast switch, done in less than $100 \mu \mathrm{S}$, caused a sudden jerk to the system as the MOT coils bounced around in their holders. Placing one's ears near the system, one could actually hear a metallic ping every time the switch occurred. An accelerometer placed on a mezzanine near the cavity clearly saw the effect of the switching (See Fig. 2.8). While a partial solution to the problem of the cavity coming out of lock every cycle was to slow down the MOT coil switching, the cavity was still very sensitive and could not be trusted to remain locked over long timescales. A more permanent solution for all cavity disturbances was needed.

\subsubsection{A Cavity Autolocker}

A good system knows when it's beat. The purpose of the cavity autolocker is to gracefully have the system leave its locking state during strong perturbations and try to re-engage the lock from scratch. There are many resources for creating autolocking systems and upon switching to an AOM cavity lock we tried to copy them [59]. Most center around either a sample and hold technique for the DC value of the PZT or a breaking of the feedback circuit on the last PI, essentially holding the feedback capacitor's charge to keep the output of the circuit the same. And while we had luck with these systems on the short timescales, disturbances lasting tens to hundreds of milliseconds would routinely fail at reacquiring lock.

Luckily the "slow" JILA loop filter was built to run in 6 modes, including but not limited to Acquire, Proportional, and various levels of PI engaged. These settings are implemented through a rotary switch working off a diode-resistor based logic system (DRL) that interfaces with many DG201 analog switches (See Fig 2.9). Creating a reliable autolocking system really just calls for creating a Finite State Machine (FSM) that follows the same rules for locking a laser to a cavity that a human follows. First, with a photodiode measuring transmission through the cavity, see if 


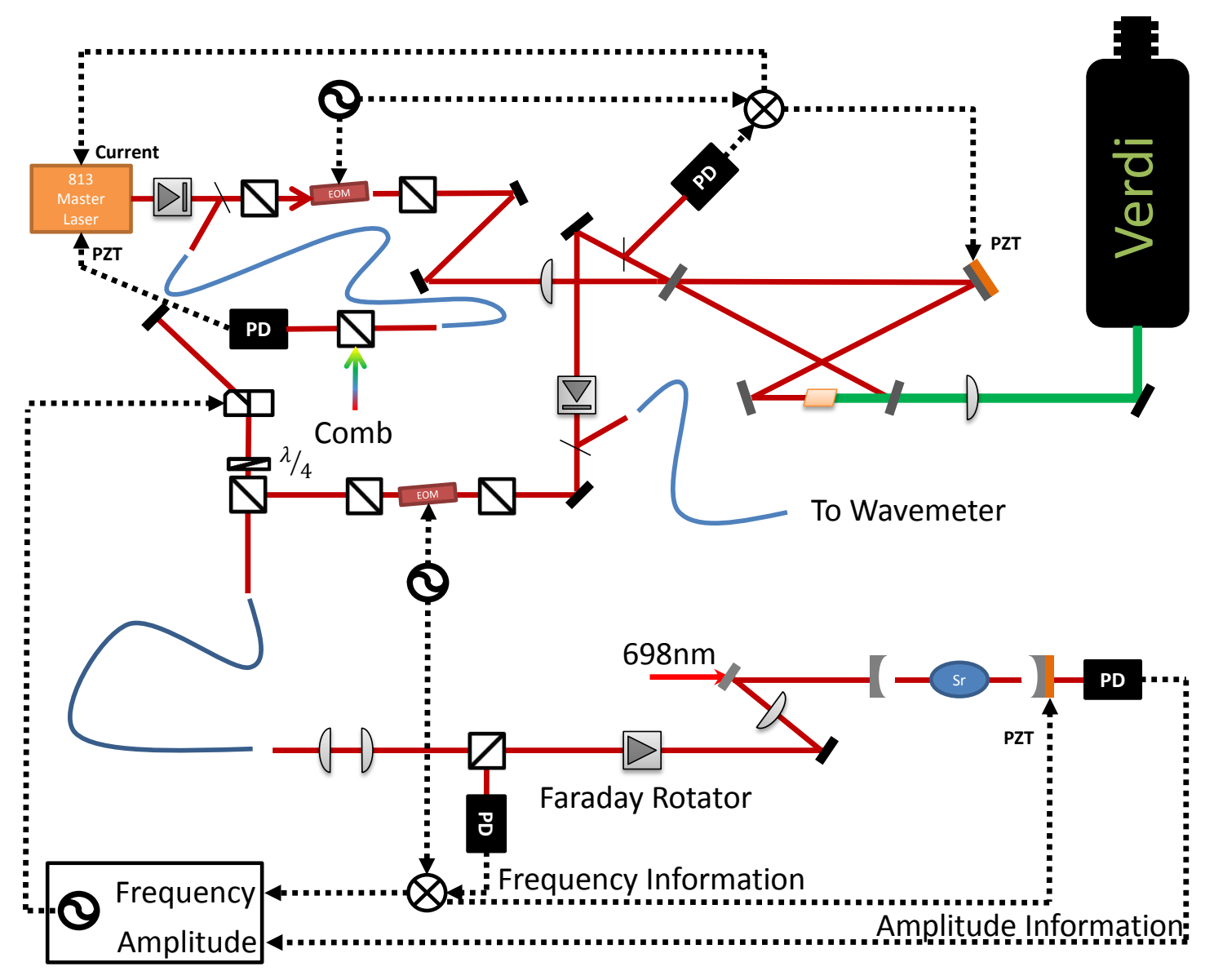

Figure 2.7: Diagram of injection locked Ti:Sapph lock to 813nm Buildup Cavity and frequency comb. Note the many frequency locks, including locks for the 2 Cavity PZTs, and the ECDL PZT, as well as the current of the ECDL and the drive of the double-pass AOM. Not all components are pictured for figure clarity. 

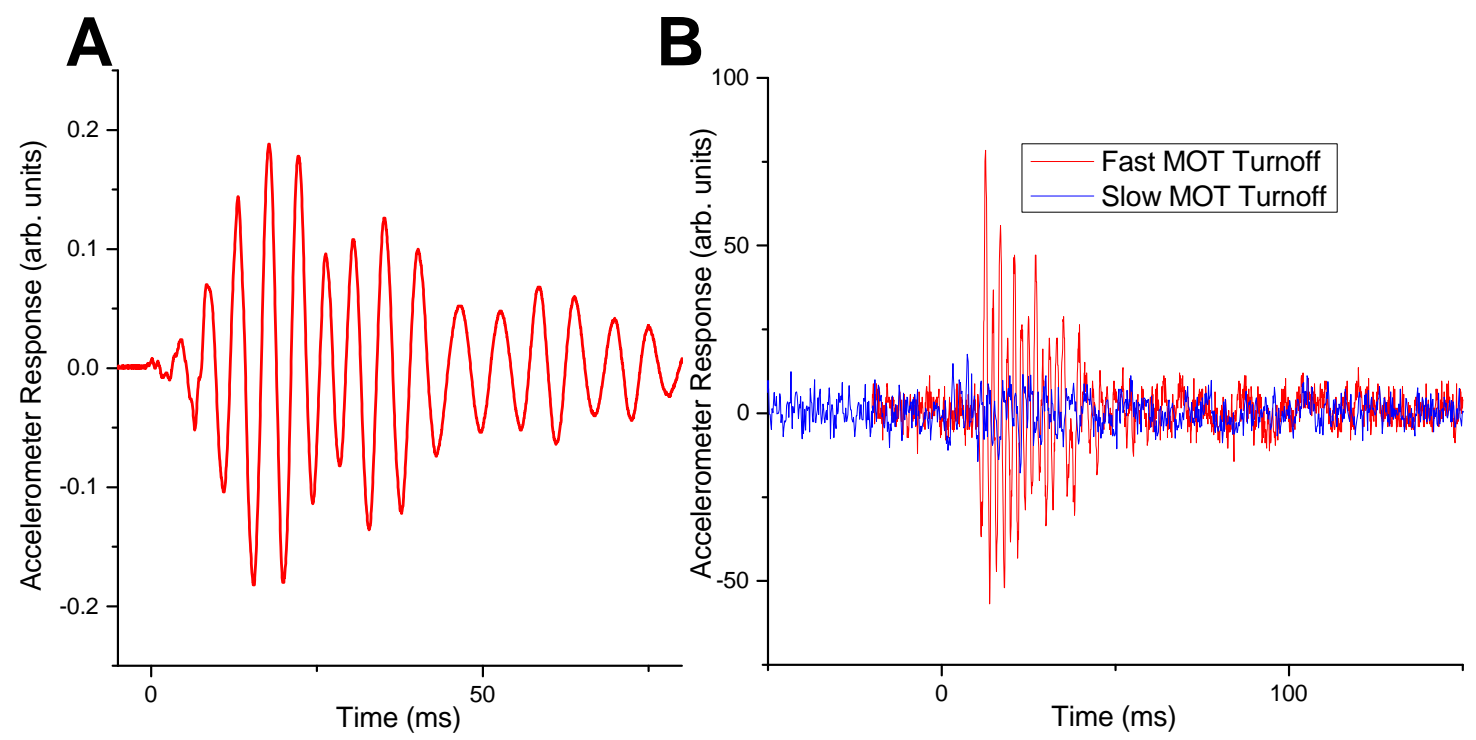

Figure 2.8: Accelerometer traces of MOT coil shutoff. A) An accelerometer is placed on a platform holding MOT optics. The voltage signal from the accelerometer is read out when the MOT coil is switched from $60 \mathrm{G} / \mathrm{cm}$ to $5 \mathrm{G} / \mathrm{cm}$ as fast as possible. A large amount of shaking is seen all around the system. B) A different accelerometer is taped onto the horizontal cavity mounts. Here one can see the difference between shutting off the MOT coils as fast as possible (in Red) or shutting off the MOT coils over $10 \mathrm{~ms}$ (in Blue) in the amount of shaking seen by the accelerometers. 


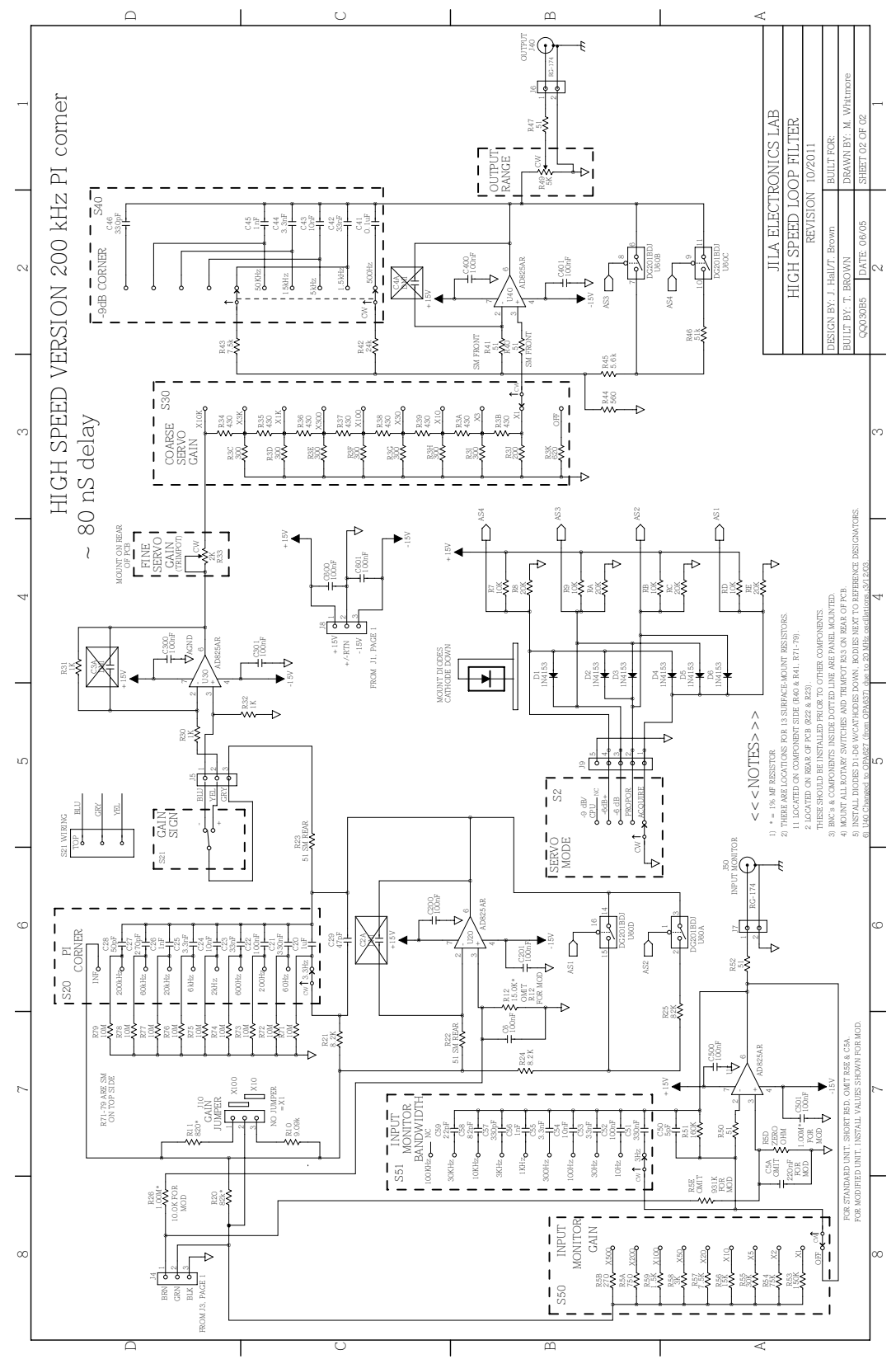

Figure 2.9: JILA Slow Loop Filter. For autolocker, header J9 is controlled via an arduino microcontroller. Further fine-grained control of the behavior of the loop filter can be obtained via bypassing the DRL logic and controlling the voltage levels of AS1-4 directly. Early attempts at sample-and-hold required this extra step. 
the transmission of light through the cavity is at a level corresponding to the cavity being locked. If so, do nothing. If not, disengage the loop filter, scan the cavity until a resonance is reached and re-engage the loop filter. A more detailed FSM diagram is shown in Fig. 2.10.

To implement this finite state machine four very simple pieces were assembled, an arduinocontrolled loop filter, a DDS capable of producing triangle waves, a voltage summer for adding the DDS signal to the output of the loop filter, and a window comparator set to give a logic high when the transmission photodiode voltage was considered high. As will be explained in Sec. 2.4 arduinoes are widely used throughout the lab because of their simple programming and ease of understanding. In this project, the arduino controls the setting of the loop filter, reads off the status of the photodiode comparator, and can phase coherently switch the DDS from a triangle wave at a frequency of a few hundred $\mathrm{Hz}$ to an effective $\mathrm{DC}$ value at $1 \mu \mathrm{Hz}$ via frequency shift keying (FSK) mode. Much like a human operator would, the system scans through a resonance by engaging the DDS high frequency triangle wave, sees the transmission go up, and stops the scan at the correct DC PZT voltage via the DDS's FSK mode. The arduino then engages the loop filter and the entire system is locked.

As this system is so single purpose, many tricks and non-standard uses of the arduino environment were used to speed up the response time of the arduino microcontroller which for the purposes of this project was an ATMEGA328. Normally, arduinos are used because they hide a lot of complexities of working with microcontrollers. The entirety of any arduino program is encapsulated in two functions; one entitled setup and the other entitled loop. Very simply, the setup function runs once upon power up, and the loop function continually loops. Arduinos also offer easy to use functions to read in or write out digital signals onto their ports like digitalRead(pin) or digitalWrite(pin, value). However, a lot of these commands are very slow compared to the clock speed of the ATMEGA chip, and there are a few simple tricks that made the autolocker work a little faster. These will make your code significantly harder to read, for those unfamiliar with their use. I'll highlight a few of the tricks here.

First, entering and exiting the stack of loop causes significant overhead that is just not 


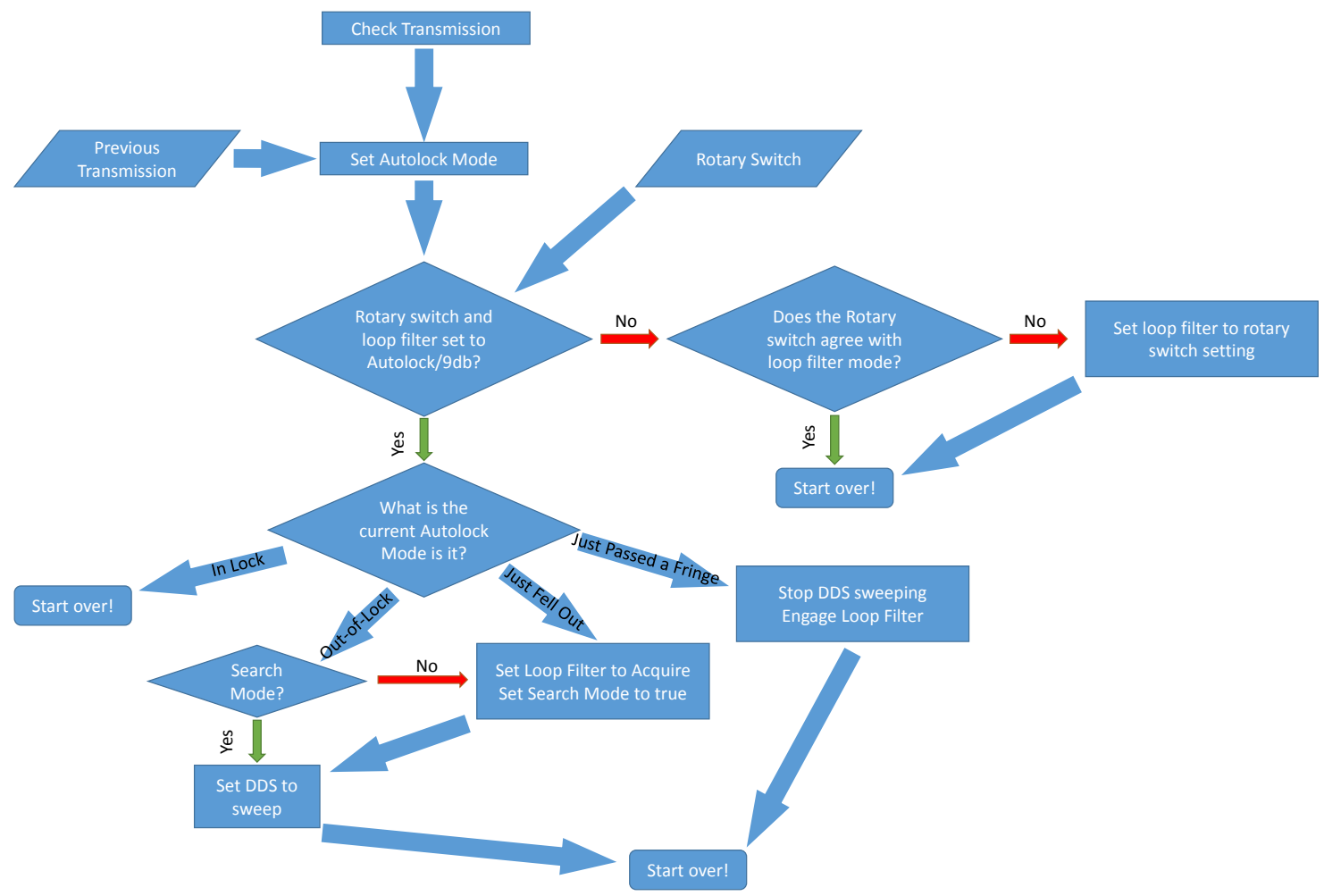

Figure 2.10: Diagram of the Auto Locker FSM. Design emphasis was placed on minimizing the delay between scanning past a fringe and trying to engage the loop filter. 
necessary for this simple a program, especially as we don't want the autolocker to miss a cavity fringe because it was busy reinitializing variables that did not need to be reinitialized. To combat this, one can put the entire program inside a while loop that cannot exit like in Fig. 2.11.

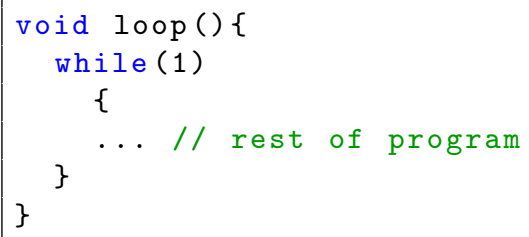

Figure 2.11: Code Snippet 1

Second, controlling or reading digital pins through the arduino commands is also a slow and time consuming operation. Instead one should directly access the port registers of the microcontroller itself, like PORTB for the output register or PINB for the input register. The use of these registers makes the code less portable as the wiring for these port registers is very chip dependent, but the speed improvements are worth it. For legibility, once the programmer has graduated or moved on from the lab, these complicated and fast operations should be rewritten with easy to understand macros like in Fig. 2.12.

\#define setModeProp() PORTD = (PORTD \& modeSetMask) I (0b00011000 \& modeSetMask)

// Set Loop Filter to proportional mode

\section{Figure 2.12: Code Snippet 2}

Finally, the interrupt handler in arduino, while convenient, is again a very slow system. Therefore, in the case of very simple programs, where every cycle counts, one should instead rely on self-programmed poling to check on outside signals changing. In the case of the autolocker, this means checking the window comparator digital signal every loop iteration with a minimal amount of overhead both before and after the poll to assure that a cavity fringe isn't missed while scanning. While the ability to create fast special purpose projects with the arduino family is nice, I will go into detail about the versatility of this family of microcontrollers in Sec 2.4. The full source code for the cavity autolocker is found in Appendix A. 


\subsection{Spectrally clean light}

When I arrived in $2009 \mathrm{SrI}$ was in the midst of a Sr-Yb frequency ratio measurement. A few months into the experiment, however, a great mystery was plaguing the comparisons. SrI, which had seemed so infalliable only a few months before was showing signs of some uncontrolled systematic shifting the line day to day on the order of $1 \mathrm{~Hz}$ or a few $\times 10^{-15}$. The most obvious culprit was the major change that had occurred between the Calcium experimental runs and those with $\mathrm{Yb}$, the switching of the injection locked Ti:Sapph laser to the simpler to use tapered amplifier system for the $813 \mathrm{~nm}$ optical lattice trap. Many experiments to determine what was causing this shift, specifically examining the TA light, were tried, but the slow speed of data taking due to needing the coordination of two completely separated experimental groups made the results hard to interpret. The comparison was abandoned, but the specter of TA shifts was present in all the minds of the Strontium gang.

The method by which tapered amplifiers could cause frequency shifts was well understood, even if the results of the first experiments were not. When pumping current through a TA, before it is seeded with coherent light, some photons can clearly be seen coming out of the chip. This light is the so-called amplified spontaneous emission (ASE). Spontaneous emission inside the chip, can easily be amplified as the light passes through the gain medium which has a fairly broad asymmetric gain curve. One can imagine that this is true for all lasing mediums, however one crucial difference remains. Most lasers operate in a regime where very little light is generated upon each pass through the medium and lasing takes place over many passes. In this regime as the seed light passes through the medium the gain gets saturated or nearly saturated, it does not matter as the now amplified seed light will make another pass through the medium. This process repeats according to the finesse of the lasing cavity, but in the end the laser's gain is saturated and there is very little amplification of stray spontaneous emission. Operation of a tapered amplifier is in a completely different regime, where only a single pass through the medium occurs, and the medium is being pumped as hard as heat dissipation can allow. The gain for a single pass remains unsaturated, and an ASE pedestal 
of light can be seen on an optical spectrum analyzer (see Fig. 2.13).

Even without complete knowledge of the ASE pedastal's effect on the atoms, two fixes for this issue were implemented. First, SrII's large atom build up cavity was colloquially known as the "filter" cavity before it was completed. Second, we purchased transmission filters with very small bandwidth (FWHM of $0.3 \mathrm{~nm}$ ) and placed them after the 813nm tapered amplifiers. So in 2011, when SrII came online as a clock, we immediately tested the effect that the transmission filter had on the SrI-SrII frequency. In Fig. 2.14 one can see the effect of removing the interference filter from SrI's system. Although it is hard to get a number from this rather preliminary test, it was clear that a large shift occurred. Subsequently, it was no surprise to hear that the SYRTE group also struggled with ASE issues in their clock comparisons. ${ }^{3}$ Before accuracy evaluations of SrII were to commence we decided that this situation of figuring out exactly what shift might be caused by the TA would be a long and circuitous road that was unnecessary. Therefore SrII rebuilt the old injection-locked Ti:Sapph system. SrI, which was going for a much less lofty accuracy evaluation, used two interference filters and a laser filtration cavity to clean up the TA light going towards the atoms. However, during the course of the SrI and SrII comparison we found that there was still a non-negligible effect being seen on SrI's AC stark shift (see Fig. 2.15). During the course of the day the shift would remain stable, but over longer timescales drifts became apparent.

As Sr clocks become more accurate a growing list of seemingly small effects will come into play, like the ASE pedastal. Reaching $10^{-19}$ will surely take considerable effort examining issues like Raman scattering inside the fiber bringing the light to the atoms or other seemingly improbable effects. It very well might be that reaching low $10^{-19}$ fractional uncertainty might require a completely new single-purpose built machine.

\subsection{Easy-to-build DDS Systems}

The work presented in this section is a direct descendant from projects done by Sebastian Blatt and Yige Lin. As the SrI and SrII systems have become more and more complicated the

\footnotetext{
${ }^{3}$ As was told to us in private communications at many conferences
} 

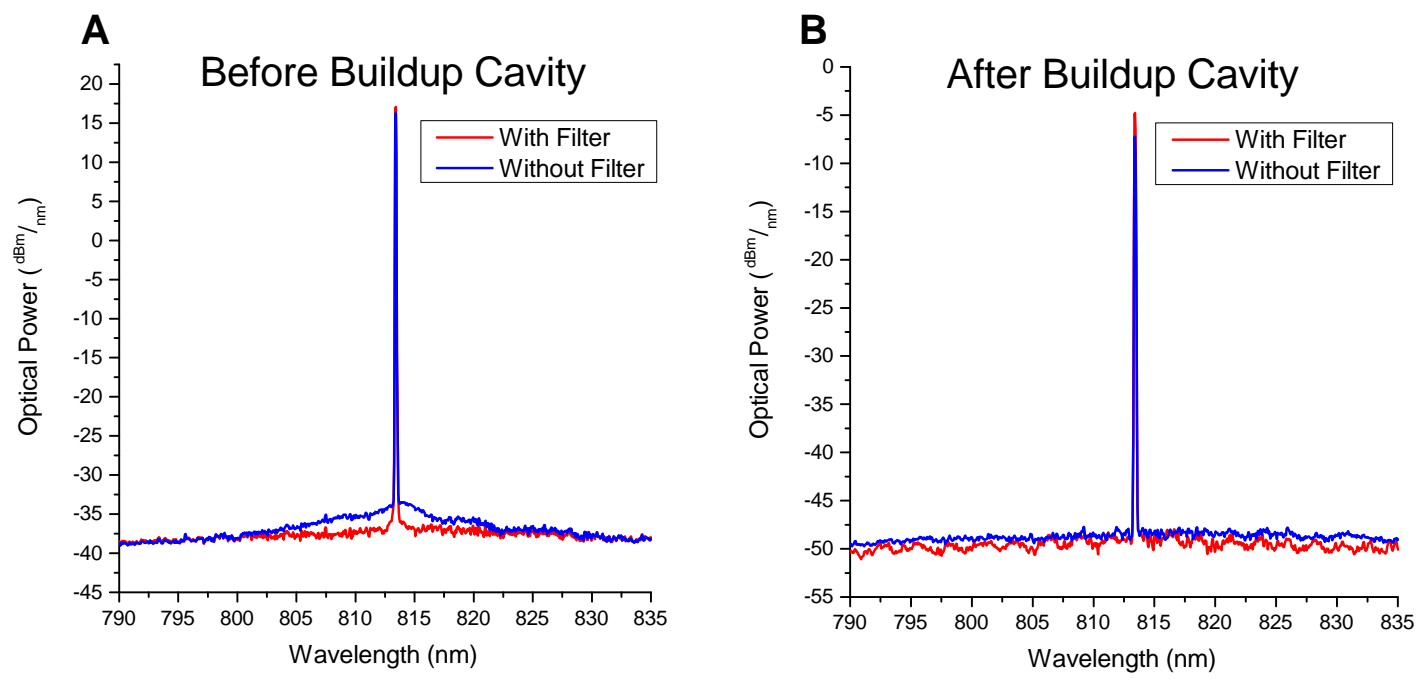

Figure 2.13: Optical Spectrum Analyzer measurements of tapered amplifier systems with varying levels of filtration. A) Traces showing the effect of transmissive interference filters before the light is further filtered by the SrII buildup cavity. B) The same measurements performed with the transmitted light of the buildup cavity. 


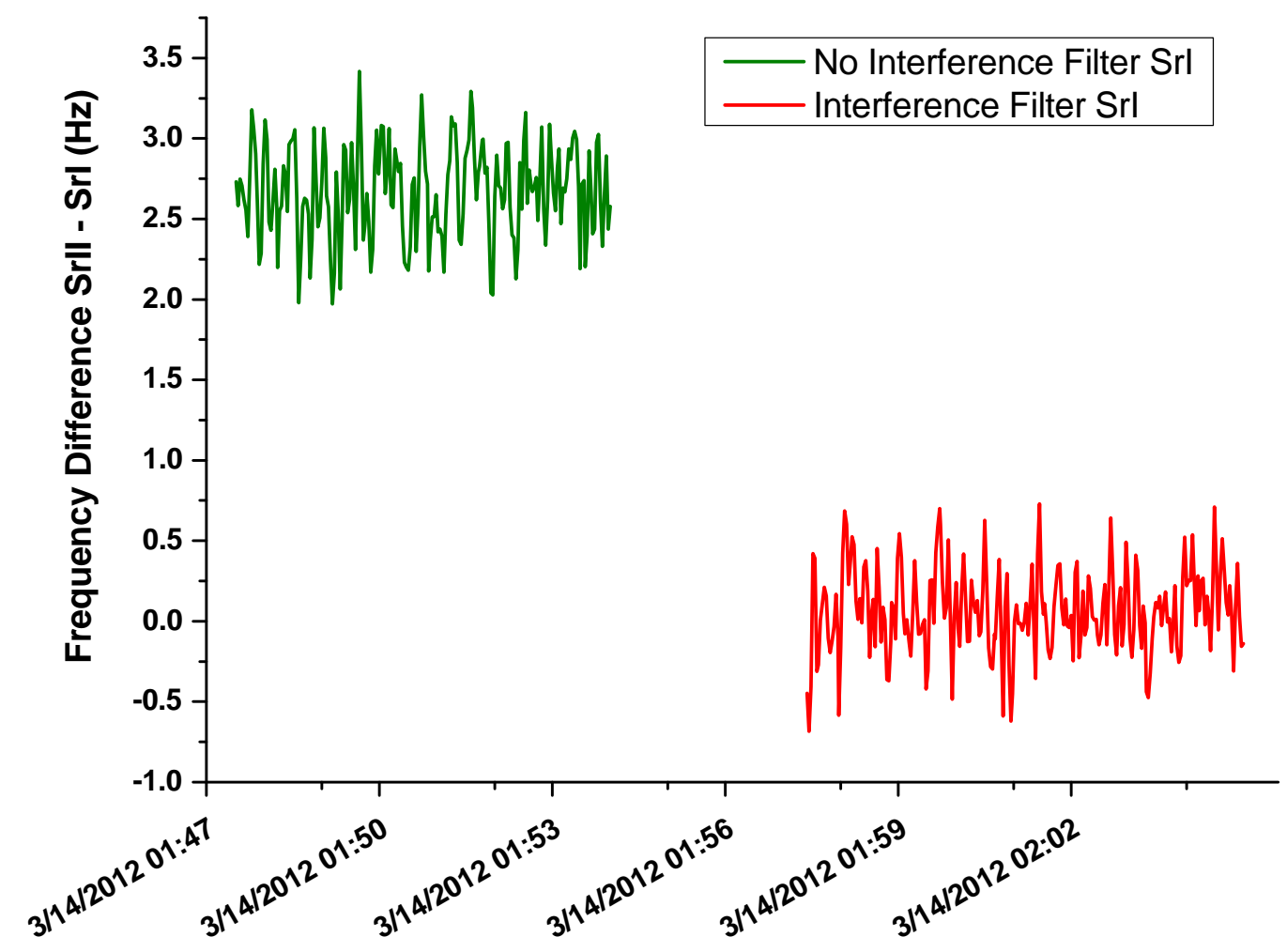

Figure 2.14: SrI - SrII frequency with and without interference filters. This test was performed on the third day of comparing SrI and SrII, and although not all systematics were stabilized at the time, the quick measurement and huge shift seemed to agree with the idea that an ASE pedastal could cause a sizable shift and was most likely to blame for issues with the Sr-Yb ratio measurements three years prior. 


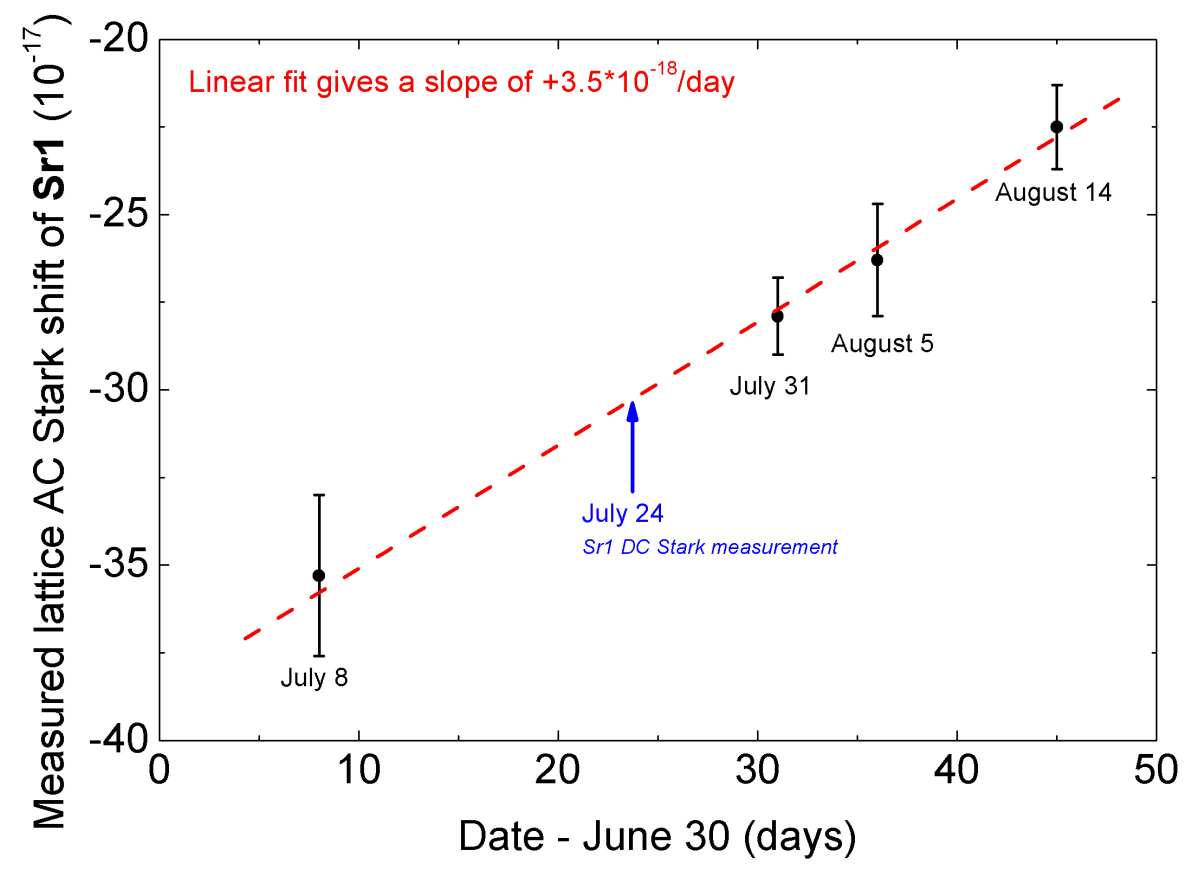

Figure 2.15: SrI TA shift drifting over the course of comparisons with SrII. Although their AC stark drifted, the absolute magnitude of the drift was still workable and allowed us to compare our clocks down to low $10^{-17}[22]$. 
need for RF sources around the lab has greatly increased. A majority of these sources need only be updated on millisecond timescales or need only switch between a few number of frequencies or phases. Rather than purchase many tens of thousands of dollars of RF signal generators we have instead created a very simple cost-effective arduino powered DDS system that is easy to replicate. Systems like these are now present in both strontium experiments, the XUV comb experiments, the stable laser experiments, and even in the ultracold polar KRb experiment.

My first project on the strontium experiment was programming PIC16f microcontrollers attached to a DAC. While this project wasn't particularly difficult, I learned a few key lessons from this project. First, unless there is a very easy to understand framework for everybody in the lab to look at and understand, code written for any project will end up being treated like a black box. Second, it is much easier to create an extensible platform for use around the lab if there are many internet resources that other students or postdocs can quickly look up. The arduino platform satisfied all these conditions and allowed for an inexpensive easy-to-program digital interface. In addition, the arduino platform can very easily be integrated into computer programs or can be used to control other digital electronics through a variety of industry standardized digital communications protocols.

In fact, the easy-to-use communications library is one of the main selling features for arduino use in lab. Many digital sensors operate using the Serial Peripheral Interface Bus (SPI) which allows one master to talk to many slave devices on the same bus. The SPI interface operates with 3 common wires going to every device, and one device-specific wire between the master and slave. ${ }^{4}$ In Fig. 2.16 one can see the topology of one arduino controlling many DDS slaves and an example of how the communications protocol works. The master arduino selects the device it wants to talk to by bringing its slave select pin low, and then sends both clock pulses and data to the slave device. If data needs to come back, the master continues sending clock pulses pulling data in on its input line.

So how does one control a direct digital synthesizer (DDS) and how does a DDS create a

\footnotetext{
${ }^{4}$ It can also operate with 2 common wires but it is less common to wire devices up like that.
} 


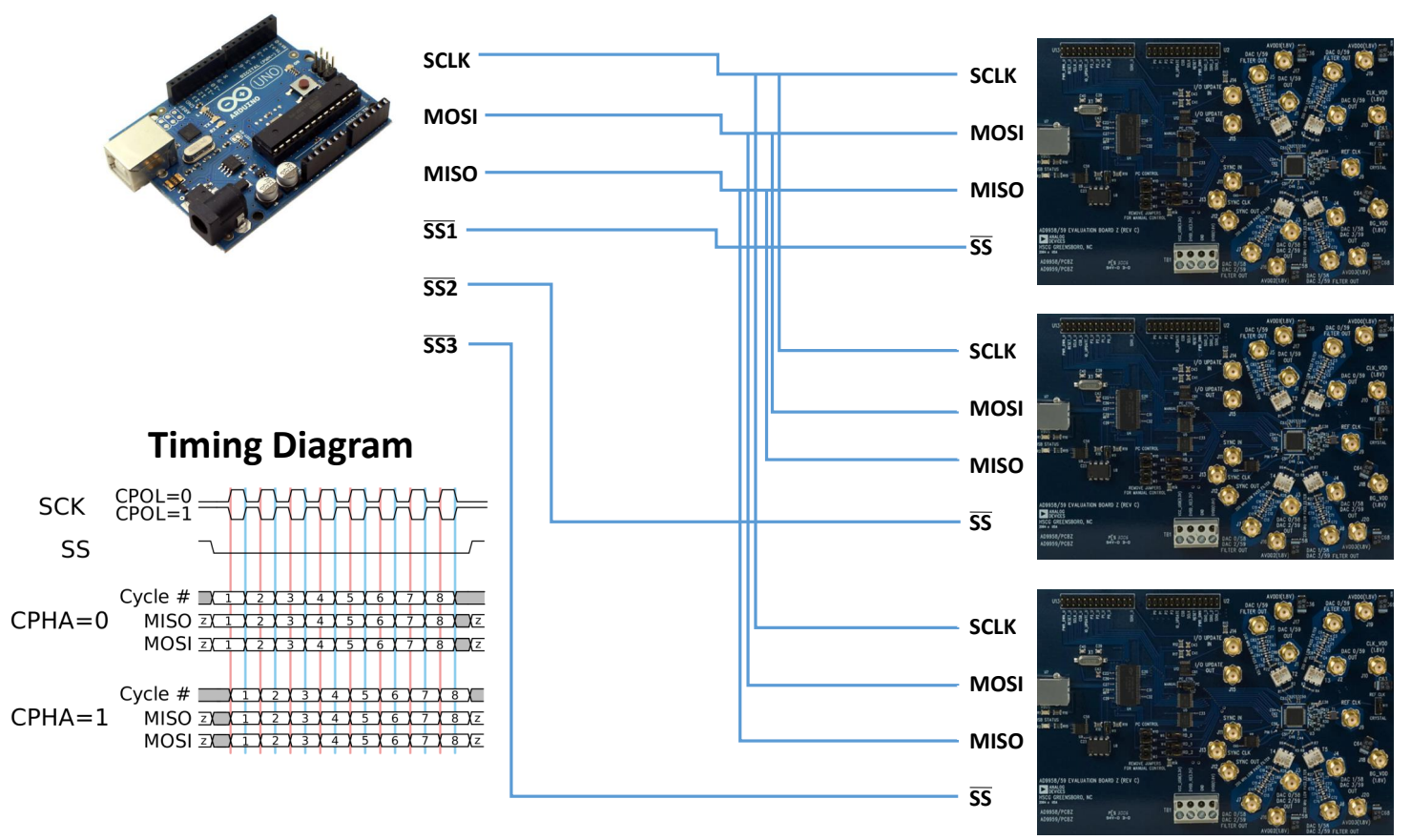

Figure 2.16: Diagram showing the topology of one microcontroller controlling three SPI slaves. The microcontroller is in charge of the communications clock and 4 different polarities of communication are possible, as shown in the timing diagram. The timing diagram is taken from Ref. [61]. 
sine wave at an arbitrary frequency? A DDS is in fact just a lookup table chip with an integrated DAC. The rate at which the lookup table is output to the DAC is set by two numbers, the master clock frequency, and the frequency tuning word (FTW). The FTW is the integer that divides the master clock which sets the output rate from the lookup table. The higher precision of the FTW the finer grained steps in frequency are possible. Of course, actually using a DDS requires much more configuration data being sent to the DDS chip as many chips come with multipliers and phase locked loops to create high frequency master clocks from stable low frequency inputs.

For high speed communications and changing parameters quickly, some DDSs offer parallel communication where data is brought in on many digital lines at a time, but programming in these situations is much more difficult and is usually reserved for FPGAs or ASICs. However, many of the DDSs we use offer the ability to program many profiles of frequency, phase, amplitude or sweeps or any number of specialized routines, making the programming part easier. But even if the programming and the construction of the hardware is easy, to be a useful tool there must be a graphical user interface (GUI). Because of the ease of talking to microcontrollers as virtual COM ports, the GUI frontend for a microcontroller controlling many DDSs can be written in any language. I chose to write the software in Matlab, a decision that will be fully explained in the next chapter. To keep the frontend code useful, the code is written to create GUIs for an arbitrary number of DDSs controlled by one arduino microcontroller. Type 2 into the initialization script and two totally seperate DDS control boxes are built. The screenshot in Fig. 2.17 shows how one can control many of the possible parameters of the DDS. This is an incredibly versatile tool that I hope will continue to be used throughout the Ye labs even after I have graduated. 


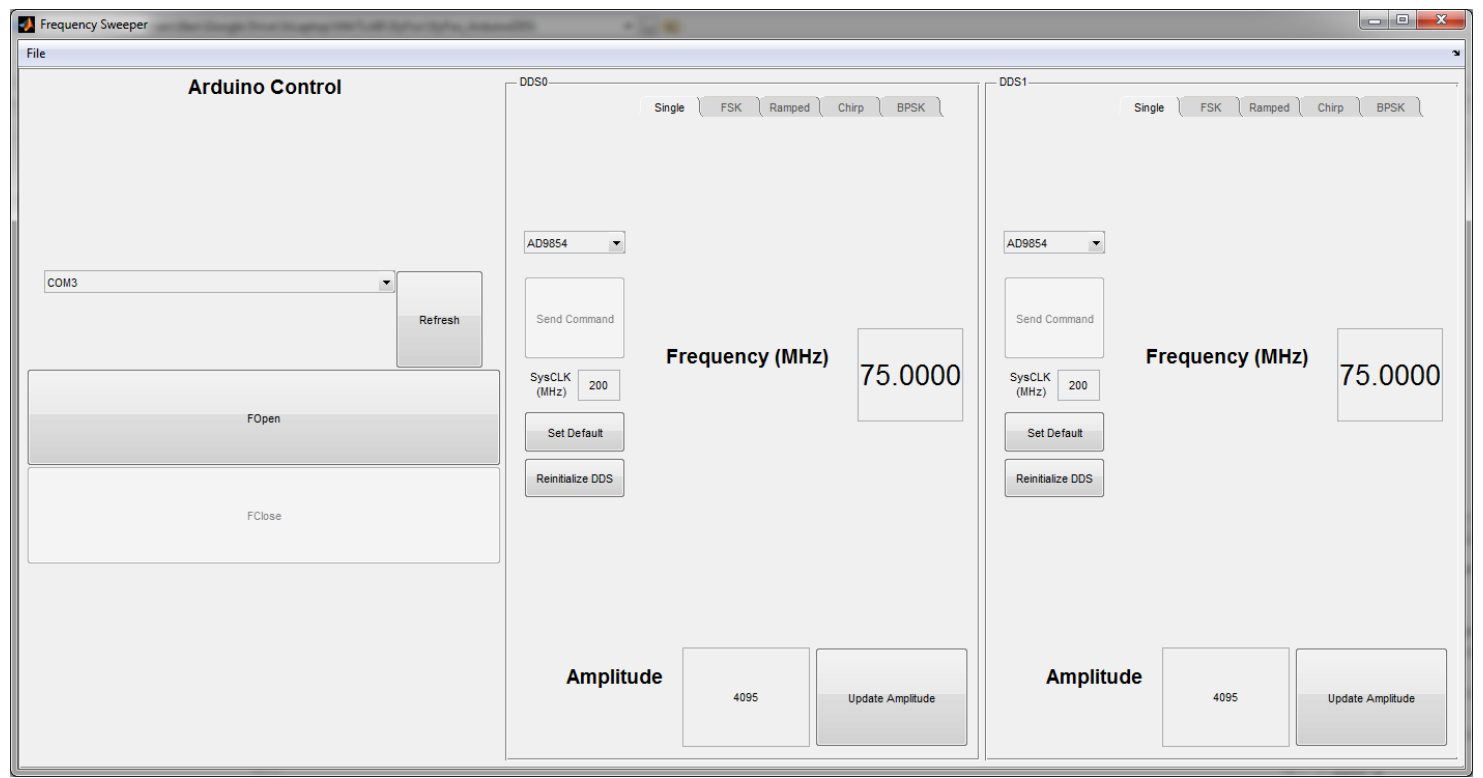

Figure 2.17: Screenshot of DDS control frontend. Interface for controlling the arduino microcontroller and the various parameters of two seperate DDSs are shown. Due to the emphasis on Object Oriented Programming, controlling an arbitrary number of DDSs does not require any additional code. 


\section{Chapter 3}

\section{Running a Clock - A Software Revolution}

There are many steps between building a clock and taking frequency comparisons. Getting all of the hardware in place is only half the battle, actually performing complicated experiments every second by orchestrating digital, analog, RF channels, and lasers without fail for hours at a time is the other half. During the course of the optical Feshbach resonance experiments we utilized a hodge podge mix of Labview, Matlab, and even $\mathrm{C}$ code. When we decided to switch the focus of the experiment towards precision measurement, the quickest fix would have been to copy SrI's Labview control code. However, after examining it closely, it seemed that the interim period of building a clock would be a golden opportunity to try and construct a flexible, extensible code framework that would allow for significantly more complicated future experiments. Watching the SrI group struggle to find work-arounds to get their Labview code to do things it was not designed to do, it seemed like the inflexibility of the system held them back. The issues I saw were not

simple to fix, and would take a concerted effort focusing on modularity and ease of programming to prepare for the challenges of running a clock that I could think of, and make it easier to expand the code when new challenges arose.

\subsection{The Experimental Sequence}

At the end of Section 1.1.1 a brief description of the experimental sequence was explained. Once the atoms have been cooled and loaded into the lattice, there are a few small details that need to be explained to create a usable clock. For the purpose of our accuracy measurements the 
MOT coils are shut off extremely slowly, and a buffer time of about $250 \mathrm{~ms}$ is instituted between the shutdown of the high current MOT field and the probing of the clock transition. During this time, the atoms are given their final cooling, as the broadband red MOT is smoothly tuned to a single-frequency red MOT and the control computer decides if further atom preparation is needed. After this optional preparation stage of optical pumping, a variable TTL pulse is sent to both the $\mathrm{RF}$ drive electronics for the final clock laser AOM and to the AOM intensity servo built to control the clock laser intensity. The clock laser interacts with the atoms with a square pulse for a variable amount of time (usually 160ms). Depending on the frequency of the clock laser and the intensity of the light the atoms will Rabi flop between the ground and excited state, with each atom ending up in a quantum superposition of these two states. Collecting the information of how the atoms react to the light frequency relative to the atomic resonance is at the heart of all our experiments.

To read out the coefficients of the quantum superposition the atoms have been placed in, we first collapse their wavefunction by turning on the blue ${ }^{1} \mathrm{~S}_{0}$ to ${ }^{1} \mathrm{P}_{1}$ light. Leaving this light on, all the atoms that have collapsed into the ground state scatter photons that we collect on a PMT, and heat out of the trap. Next, we turn on the repumper lasers that connect the ${ }^{3} \mathrm{P}_{0}$ and ${ }^{3} \mathrm{P}_{2}$ state with the ${ }^{3} \mathrm{~S}_{1}$ state allowing the atoms to eventually fall back to the ground state via the ${ }^{3} \mathrm{P}_{1}$ state. Once again the blue light is turned on and the population of atoms in the ground state are counted by capturing some of the scattered fluorescence. Capturing these PMT signals is an analog signal digitizer card on our data computer.

Scanning across the clock transition one could see many possible resonances depending on the nuclear spin state occupation of the atoms and the magnetic bias field vector. One might see all the $\pi$ transitions between ground and excited hyperfine states for an unpolarized sample with a bias field, a single clean optically pumped $\pi$ transition, or without a bias field a mess of overlapping lines leading to a broadened unpolarized line. With the excitation signal read from the ensemble of atoms, which concludes one cycle of a clock measurement, the sequence starts over and a new set of atoms is loaded and cooled. This repetition of TTL signals is created by a commercial FPGA board that does not know what kind of scan has been completed nor does it know what the frequency 
of any RF oscillators are, it just keeps repeating the same sequence of TTLs over and over again. To turn this into a smart system something needs to manage all the details, and connect those details with the measurement results. The software that performs all this manipulation and data taking will be explained below. To connect the software to what is happening in the real world, we look no farther than the digitizer card that reports once a second after its data buffer is full. This seemingly innocuous "Buffer Full" message is the key to connecting what is going on in the real world with what is going on in the software's model world.

\subsubsection{Synchronizing Two Clocks}

Before I go on to explain the software, another hardware issue remains. The SrI and SrII experiments were built to be independent, but data taken during comparisons between SrI and SrII needs to be compared precisely without any timing slop. This is especially true for the case of synchronized detection where some amount of common-mode laser noise is removed $[28,62]$. While, at first, accomplishing this task seemed easy enough by having one control FPGA trigger the other FPGA, data analysis showed some very peculiar issues. Each setup would timestamp the data they had gotten with the time read off by the computer operating system. Even for datasets that were known to be taken with both experiments synced up to the ns level, the timestamps between the two computers showed cyclical drifts on the order of $900 \mathrm{~ms}$. As the duty cycle of the experiment was very close to this length in time, it became a real problem to try and 'sync' up these two data streams for analysis.

A solution was needed to simplify the timestamping problem and not allow experimenter bias into the data processing. Our first solution was to use an arduino with an ethernet shield add-on. One of the FPGAs would trigger a single pulse for each experimental run and the arduino would count these pulses. Whenever either experiment wanted to add a timestamp to their most recent data they would ask the arduino over TCP/IP what the current pulse number was. While this seemed like a simple solution, the inclusion of the ethernet shield to allow communication between the arduino and both strontium control computers created a strange confluence of blocks for the 
arduino. Issues of communication between the arduino and the ethernet microcontroller, and blocks when both computers were trying to talk to the arduino caused some pulses to be missed by the arduino resulting in noncontiguous datasets. As most of these issues stemmed from what were essentially threading problems, it seemed reasonable that a commercial product, like a frequency counter would have most of these issues worked out. Surprisingly, the task of continually counting pulses while being asked what the total is is not a common capability of many frequency counters. Luckily, after searching we found that newer Agilent counters (e.g. the 53220A) could perform this function. Overall this system worked fairly well and allowed easy frequency comparisons to be analyzed between the two strontium experiments.

\subsection{The Slyfox Software Suite}

The Slyfox software suite is a collection of programs I wrote to control many aspects of the data taking process. The use of a common framework across all the programs in this suite of software is to allow maximum code reusability and fast extensibility. Many of the smaller pieces of the programs are written with object oriented programming (OOP) in mind to allow for easy access to hardware devices like arduinos, DDSs, analog outputs or software devices like other computers via TCP/IP communication, PIDs, and data automation programs. The GUIs in all of these programs are separated out from the implementation with the intent to preserve a Model-View-Controller (MVC) backend agnostic realization that allows for a clean separation between coding tasks. The choice to program in Matlab for the control software for SrII was a conscious decision to minimize the learning curve for future students who might not know how to program. For the benefit of future generations of strontium students, I'll include explanations below of the concepts employed in SlyFox's various programs and give concrete examples of how the software uses these techniques. All code is accessible online hosted on its own Google Code page at https://code.google.com/p/slyfox/. 


\subsubsection{An Object-Oriented Programming Primer}

To explain OOP it might be useful to examine an example of how one would describe a real world setting with OOP principles. Borrowing a page from the Advanced Placement test, let's examine the structure of an ocean. Our pretend ocean will have an environment, the sea, and things that live in this environment like fish and sharks, and we will be charged with showing the fish moving around and the sharks hunting the fish. The key insight that OOP will bring to the table is that in this programming scenario there are many pieces of modeling code that are seemingly redundant. There will be many fish and sharks in the sea, and even if they are not all exactly the same, they will differ only in small details which can be individually cataloged, and their behaviors will in fact be nearly identical. Furthermore, while we might think of sharks and fish as very different, it's plain to see that we might model their movement in the exact same manner. So it seems clear that whatever data structure or form these fish take should somehow make the relationship between fish and sharks evident as well.

While OOP can be implemented in almost any language, I will discuss the framework used in Matlab and Java as they have many similarities ${ }^{1}$. We've already discussed that any representation of a fish (or shark) might include details about an individual fish, which will be known as properties, and also behaviors that the fish has, which will be known as functions. The same can be said about a shark, that it is somehow a special case or a member of a subset of fish. To describe our fish we will write a fish Class. This Class file will list all the attributes and behaviors we could ever want a fish to have. In our example, fish will have three public properties a color, a position and an environment, and will have a few public functions, including swimForward, swimBackward, swimLeft, swimRight, and a constructor and deconstructor functions. These latter two functions are included in every Class file.

To fill our pretend sea with fish, one will simply instantiate many objects created from the Class fish. Instantiation is the process of calling the constructor functions, where the programming

\footnotetext{
${ }^{1}$ While I will use the word and explain the concept of an Interface, Matlab does not actually implement this idea, as Matlab is much less stringently typed than Java.
} 
language carves out a piece of computer memory that holds copies of all the data in fish that belong to each new fish object. Instead of keeping a list of the color of each fish in your main program, when the fish are being constructed, one simply gives each fish a color. If for some reason you want to know the fish's color one simply needs to access that chunk of information in each object ${ }^{2}$. But how does this work for a shark? As I mentioned before, in our simulation, a shark will be very similar to a fish. In fact, it might have all the same public properties and maybe even the same public functions. The only difference might be in the addition of a few functions or maybe in the implementation of some of the already present functions. Most object oriented programming languages have this extension or hierarchy idea built in. What would be created is a Class shark which "extends" the Class fish. Shark is now a subset of fish, allowing it to act and have the same memory structure and functions as a fish. In strongly typed languages this hierarchical structure can incorporate more relationships than just the idea of a subset or special case. By implementing interfaces one can create certain "types" of objects. For example fish and sharks might both include a function Swim, but so could turtles, and interfaces are used to establish this common Swimmable behavior.

While this complicated array of relationships and hierarchies between objects might not seem very important, let's examine why this might make our implementation of an ocean more efficient. Imagine that our program has filled its pretend ocean with fish and sharks of various colors and each object knows its position in our pretend ocean. Running the simulation of our ocean might be a very simple matter of telling each object to "move". Rather than have the main program deal with all the complexities of repositioning each fish, arbitrating whether the fish objects can overlap at the same position, or causing the sharks to eat the fish, all our main program does is tell each object, maybe in a random order, to move. All behaviors and interactions between objects will be controlled at the object level. Think of how simple our program's structure has become, we would not have to write complicated routines for moving fish and then moving sharks, or carefully orchestrating each object's actions, instead it has all been structurally simplified. At the object

\footnotetext{
${ }^{2}$ Some languages might require one to program in "get" functions for retrieving properties about each object.
} 
level there is still code to be written, but the behaviors are much simpler to compartmentalize and write. So not only does OOP allow for easy duplication of common resources, it can also be a powerful tool for creating structurally simple programs by offloading tasks into well defined objects.

\subsubsection{An Example: The Slyfox Frequency Synthesizer}

Lets examine how the slyfox program uses OOP to create a versatile frequency synthesizer for use in frequency scans for the clock laser, optical pumping light, or sideband cooling. First off, let's think about the requirements this interface for hardware instruments is going to need. One, we are going to need to control a lot of different RF synthesizers with this software. This meant that the interface needed to agnostically talk to different synthesizers using different commands. Two, setting up communication between the interface and the instrument should only take a second and should be very simple. And three, the interface must have a clean and simple user interface (UI) that allows each instantiation of this object to have very simple to use controls that can be placed automatically in the main program's control window. To begin with, I'll show you a few of the basics of OOP that are implemented in this frequency synthesizer, and how these requirements are all addressed.

classdef FreqSynth < hgsetget

\%FREQSYNTH Simple interface to control HW instrument

Figure 3.1: Code Snippet 1

The code in Fig. 3.1 begins with the keyword classdef which tells the interpreter that this file will be defining a class by the name of FreqSynth which will be a subset of the class hgsetget. As can be found buried deep within the Matlab documentation, the hgsetget class extends the handle class. This not only allows the use of set and/or get methods to change the properties of an object without having to program those all by hand, it also makes sure that this object will be pass-by-reference versus the default pass-by-value. Making these objects pass-by-reference is a necessary addition to the FreqSynth class because references to these objects will need to be used by multiple other resources like frequency sweeper or frequency locker programs. Passing these 
objects by value would create multiple interfaces to the same hardware object which would not have the same properties or settings.

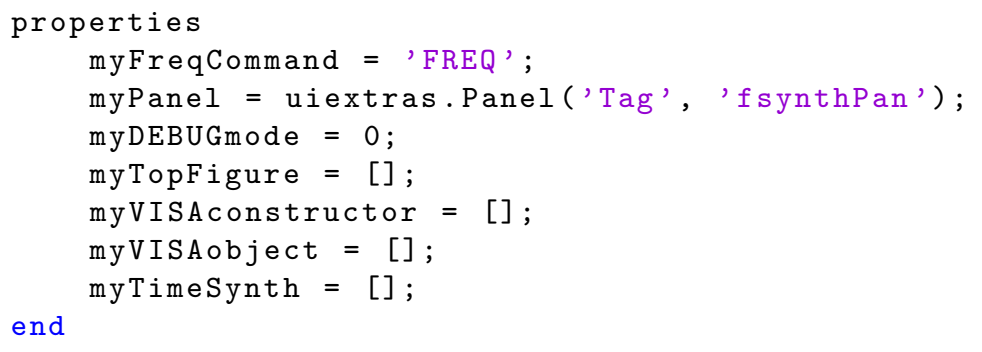

Figure 3.2: Code Snippet 2

Following the class declaration are the properties (in Fig. 3.2), data specific to each instantiation of the class ${ }^{3}$. The three most interesting properties to point out are myPanel, myVISAobject, and myFreqCommand. MyPanel is a UI panel that will hold all dropdown menus, buttons, and text inputs. MyVISAobject is a virtual instrument software architecture (VISA) which allows socket-like communication between serial, TCP/IP, USB, and GPIB ports. This allows the program communicate with any hardware connected to the control computer regardless of how the connection is made. The string myFreqCommand will allow us to easily change the behavior of the hardware device, as explained later in this section. With the individual characteristics of each object laid out, let's examine the object's constructor to see how it builds a FreqSynth object.

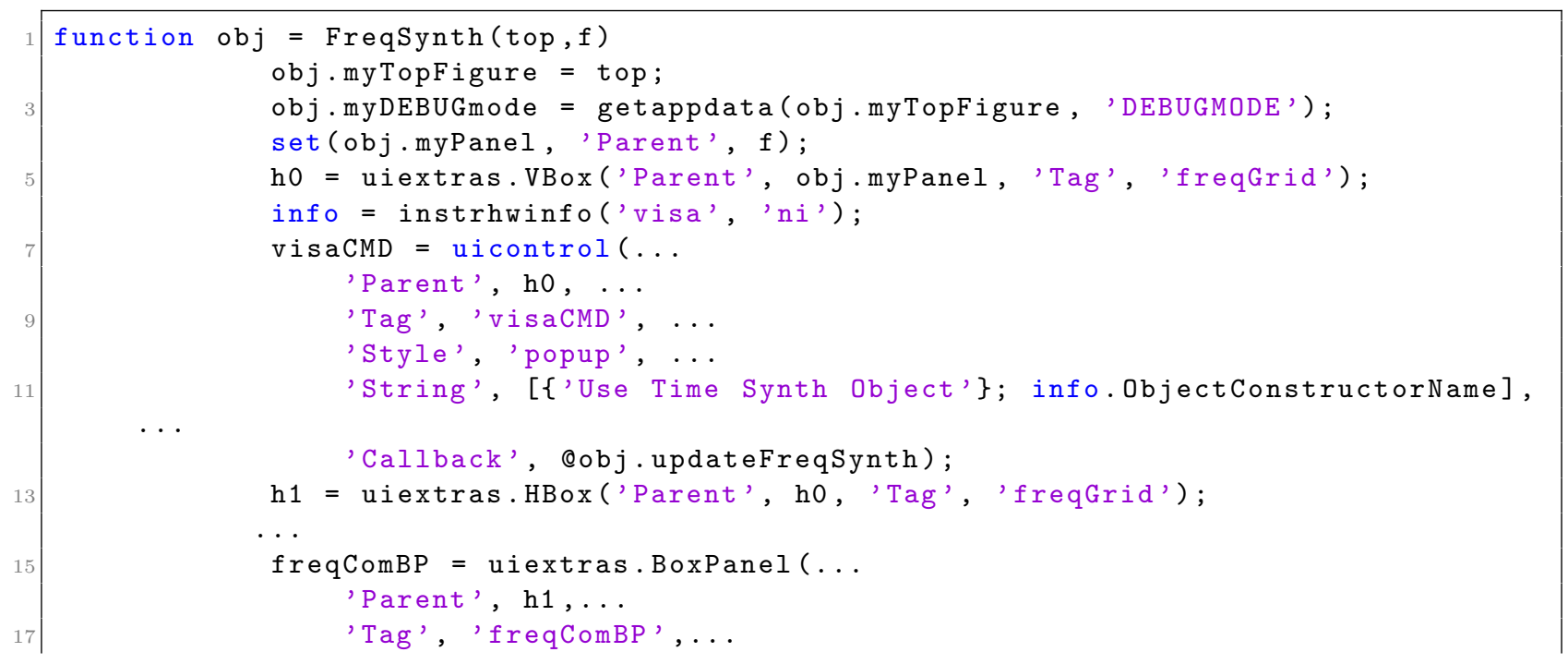

\footnotetext{
${ }^{3}$ It is possible to make variables that are not instantiation specific, these are denoted by the use of the modifier static.
} 


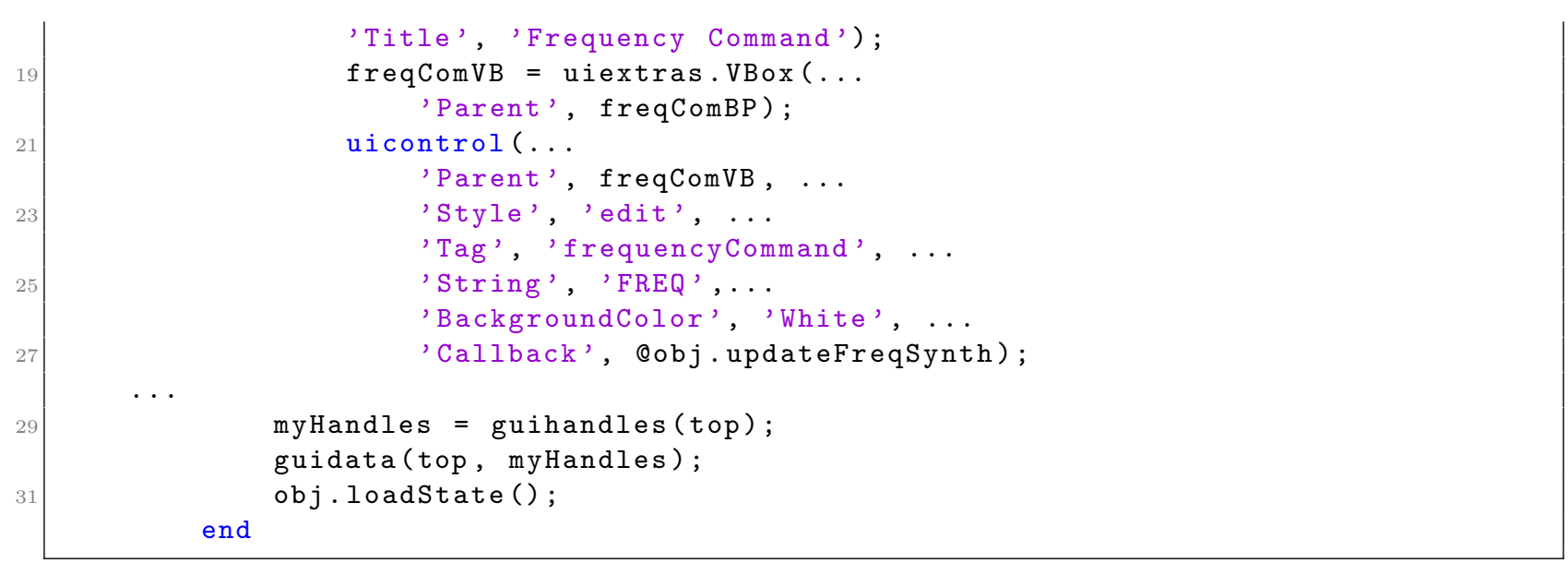

\section{Figure 3.3: Code Snippet 3}

For brevity's sake, I have removed some of the extraneous UI code (in Fig. 3.3) to allow us to focus on the important points included in this constructor. Overall, it should be noted that many of the commands in the FreqSynth constructor are in fact calling other constructors. For example, all of the function calls starting with uiextras are creating UI objects which get placed inside the obj.myPanel which in turn gets placed inside of the Panel $\mathrm{f}$. The use of this uiextras package allows for a dynamically sized and placed grid of UI elements, which makes GUI programming significantly less time consuming than placing all the elements by hand. Furthermore, this simplifies the difficulty of creating an arbitrary number of FreqSynth objects, as all one needs to do is carve out a Panel to hold the new object and pass a reference to it in the constructor.

Lines 6 and 11 of the constructor in Fig. 3.3 create a list and dropdown menu for all computer connected hardware devices. Choosing which instrument to talk to is accomplished by just choosing an element from the dropdown list. In addition, line 31 is used in conjuction with a saveState function to create a default selection for the communications dropdown menu and other UI elements. Finally, to create large hardware and function compatibility, lines 21 to 27 create a text box allowing for the command that will be sent to the hardware to be entered, the myFreqCommand string. For example an SRS function generator might expect to receive a command like FREQ 10000000 whereas a dual channel tektronix DDS might expect the command SOUR2:AMPL 0.0 for scanning the second output channels' amplitude. Rather than focus on the minutiae of initialization and error catching, we'll instead focus on exactly how this last functionality is in fact implemented. 


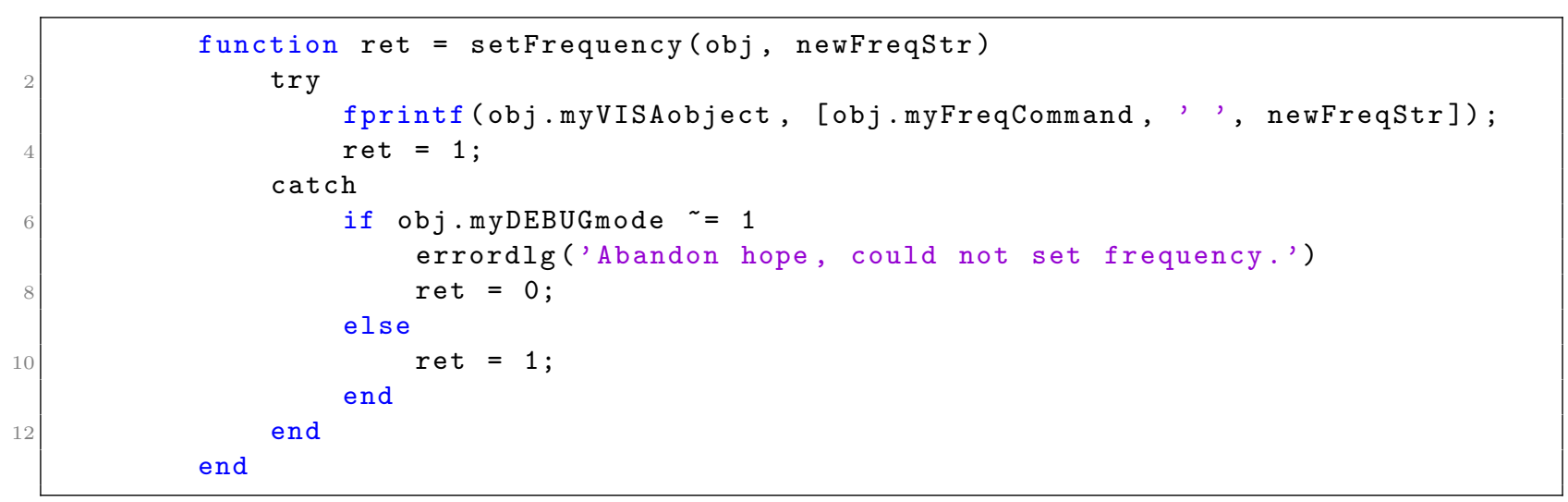

Figure 3.4: Code Snippet 4

The function setFrequency in Fig. 3.4 is in some ways an all-purpose communications passthrough from the program interacting with the FreqSynth object and the physical hardware. Surrounded by error catching and debugging code, this function writes to the port obj. myVISAobject via the function fprintf a string of text it creates from the substring obj.myFreqCommand, we discussed previously, and the substring that is passed into the function call, newFreqStr. Creating easy access to this function for a variety of programs is in some sense the sole purpose of this entire object. Furthermore, it allows for easy automated software control of any output parameter of a function generator that can be characterized by a number.

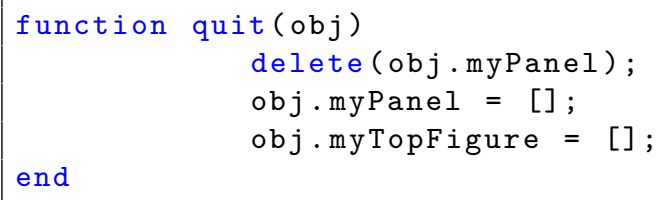

Figure 3.5: Code Snippet 5

Finally, to conclude our exposé on all the important features in an object we have a destructor in Fig. 3.5. This piece of code essentially releases all the pieces of memory that the computer might not realize were associated solely with the object. In the Matlab language, the function delete knows how to free all base pieces of data, it is up to the programmer to make sure that references to other objects and instantiations of other objects get correctly freed.

Throughout this examination I've tried to highlight 5 mainstays of OOP, constructors, properties, functions, and destructors. With knowledge of how a simple hardware interface can be constructed, it's time to see how to orchestrate many objects to create complicated behaviors like 
locking a clock laser to the atoms and routinely going into a pseudo atom-debug mode all while staying synced with another strontium experiment.

\subsubsection{Bringing it Together: The Slyfox Frequency Locker Program}

My hope for this section is not only to show how one might create a complicated object oriented program, but also to fully explain how a locking sequence works with the intent that anybody reading this will be able to come up with their own locking program to accomplish whatever measurements they might need. SrII's control code was built specifically with this objective in mind; a completely new custom locking sequence should take no more than 30 minutes to write and hopefully a similar amount of time to debug.

To initiate the program one runs one simple function FrequencyControlMain. The function is actually quite short, and all it does is instantiate a few objects as can be read below.

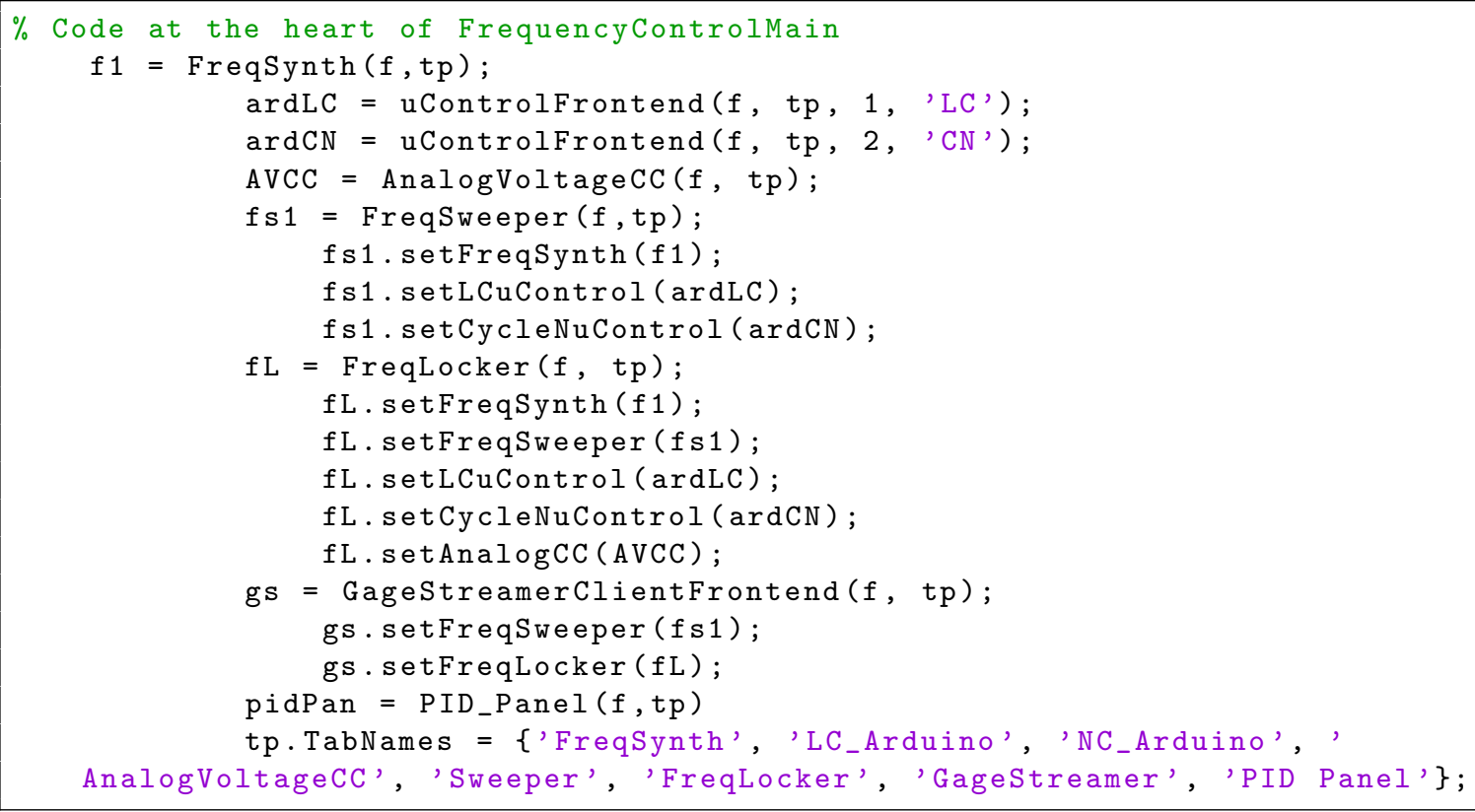

Figure 3.6: Code Snippet 6

All of the objects instantiated in Fig. 3.6 are used to interact with hardware surrounding the experiment or to run programs that control these hardware objects. For example, ardLC and ardCN are interfaces for talking to an arduino in charge of the liquid crystal waveplate or a cycle number counter. AVCC is an interface to an NI 6733 analog output card used to control magnetic fields or 
MOT loading rates. Finally, fs 1 and $\mathrm{fL}$ (line 6 and 10 respectively) are used to orchestrate all of these hardware elements for the purposes of taking sweeps or locking the clock laser, respectively. Notice how references to all the hardware interfaces are passed to the programs that will utilize these interfaces as in line 11. This main function just instantiates the objects, and tells them to take up residence in tp which is a tabbed panel UI object by passing a reference into their constructor. This is yet again another simplification enabled by using OOP as no effort is wasted on figuring out how and where to add all the UI elements as the layout manager built into the uiextras package is algorithmically placing and resizing all the buttons, text fields, and menus for us.

Let's now focus on the frequency locking program. The full code line count for Freqlocker is about 4600 lines, so to explain how the majority of the code works I will judiciously be cutting out a lot of non-important code. Let's begin with the FreqLocker properties .

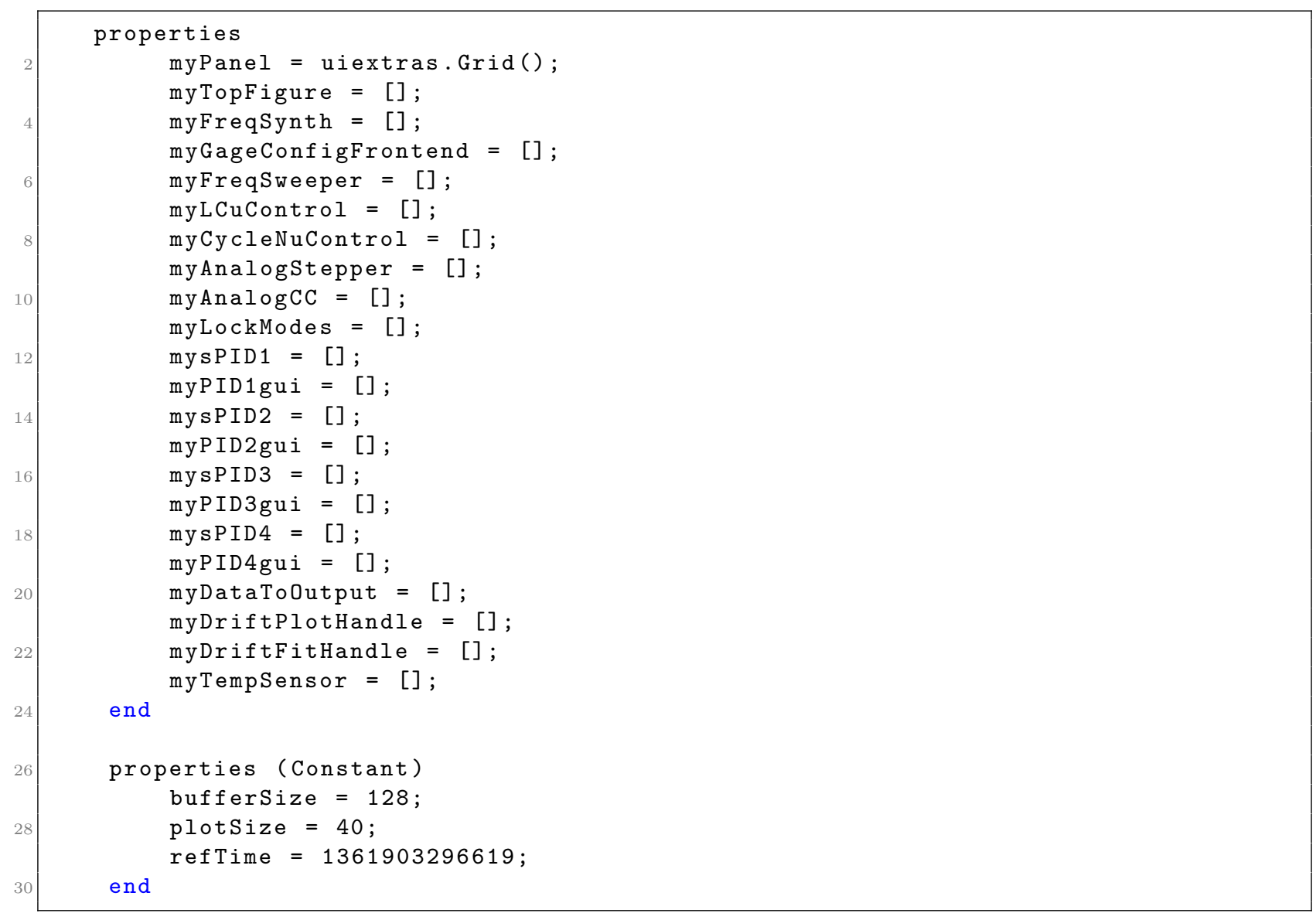

Figure 3.7: Code Snippet 7 
Hopefully many of the handles in Fig. 3.7 are self explanatory. The Constant or static properties are ones that do not need to change between instantiations of the object, and are mostly aesthetic information used to make the plots look nice. There are two new kinds of objects that are very important to the locking process that I have not shown the reader yet, the seriesPID and the PID_gui objects. These objects are exactly what they sound like, a seriesPID is a very simple object used to calculate the response of two standard form PIDs in series. Where of course, the standard form is written as in equation 3.1. And the PID_gui object was written to keep the PID and seriesPID objects to be as simple and versatile as possible without getting caught up in UI code.

$$
C=K_{p}\left(e(t)+\frac{1}{T_{i}} \int_{0}^{t} e(t) \mathrm{d} t+T_{d} \frac{d}{d t} e(t)\right)
$$

The instantiation of 4 of these completely independent objects will be the backbone of some of the most complicated locking sequences, and in the future with more experimental conditions under digital feedback instantiating more digital locks is just a few simple lines of code. The constructor for FreqLocker places and/or creates many of the objects listed in the properties leaving a tab panel like that shown in Fig. 3.10. To allow FreqLocker access to already created objects like a FreqSynth, handles are passed to the FreqLocker via helper functions after its construction like in Fig. 3.8.

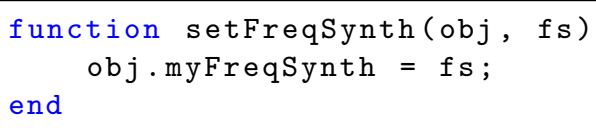

Figure 3.8: Code Snippet 8

With all the pieces set, let's examine how the program initiates a lock and runs the system. After taking a Rabi scan, guesses for where to start each of the four PIDs are loaded into the text boxes on the right of Fig. 3.10 by the user. The user chooses which locking protocol to use from a dropdown list and hits "Start Acquire". This button's callback below is fairly simple as it offloads a lot of work to a different function.

function startAcquire_Callback (obj, src, eventData)

myHandles $=$ guidata (obj.myTopFigure); 


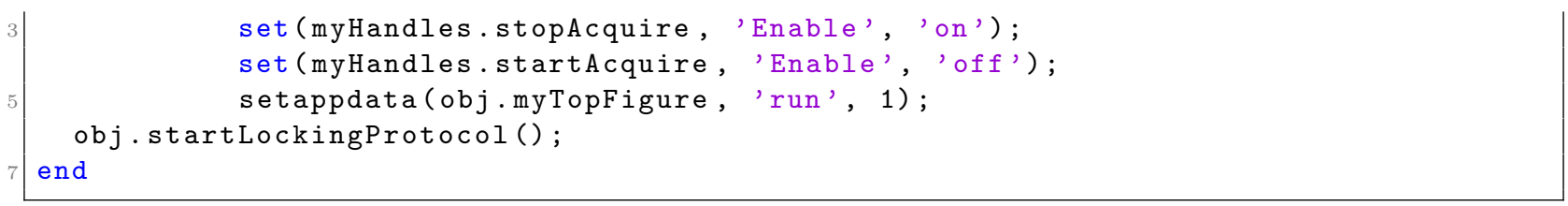

Figure 3.9: Code Snippet 9

The startLockingProtocol reads off which protocol has been selected from the dropdown menu and launches that protocol's initialization function. For our example using the locking protocol "4 PID Locks with Rezero" it calls the function obj.start4PeakLockRezero_initialize(). In the future when new protocols have been written, not only will they need to be added to the dropdown list in the FreqLocker constructor, they must be added to this launching function as well.

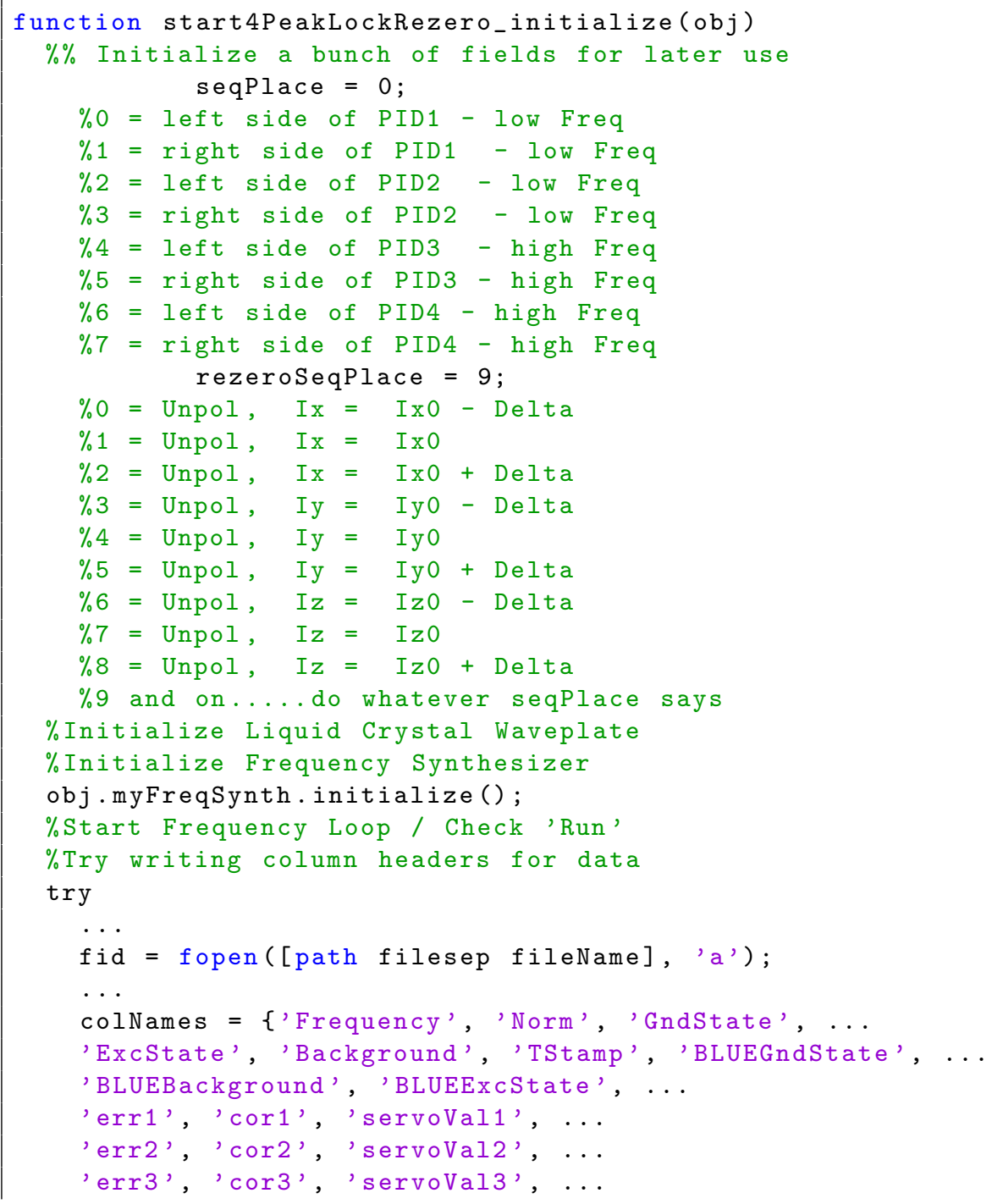




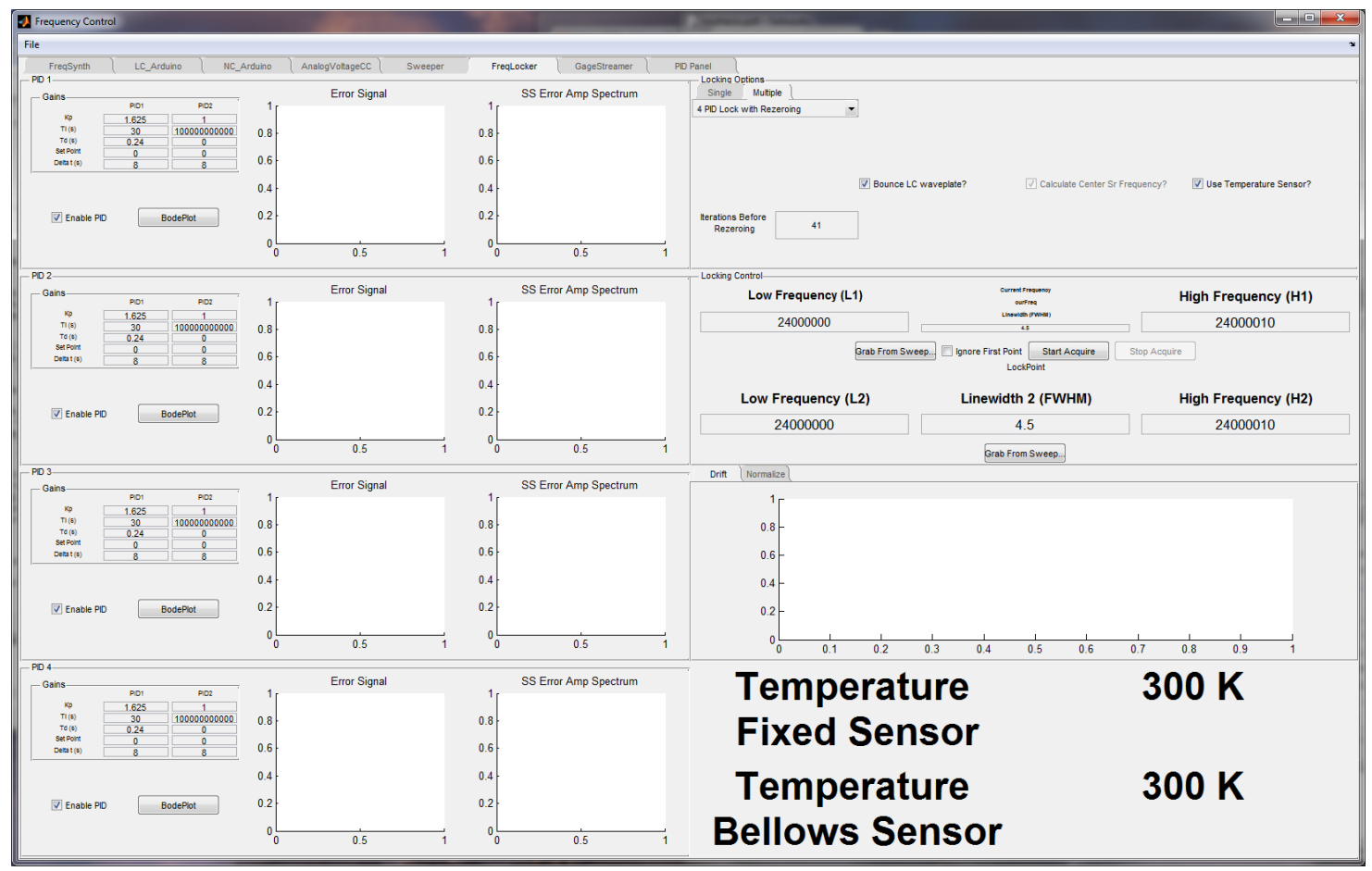

Figure 3.10: Screenshot of the Frequency Locker program. Note the many independent PIDs on the left, and the controls on the right for choosing the locking protocol. 


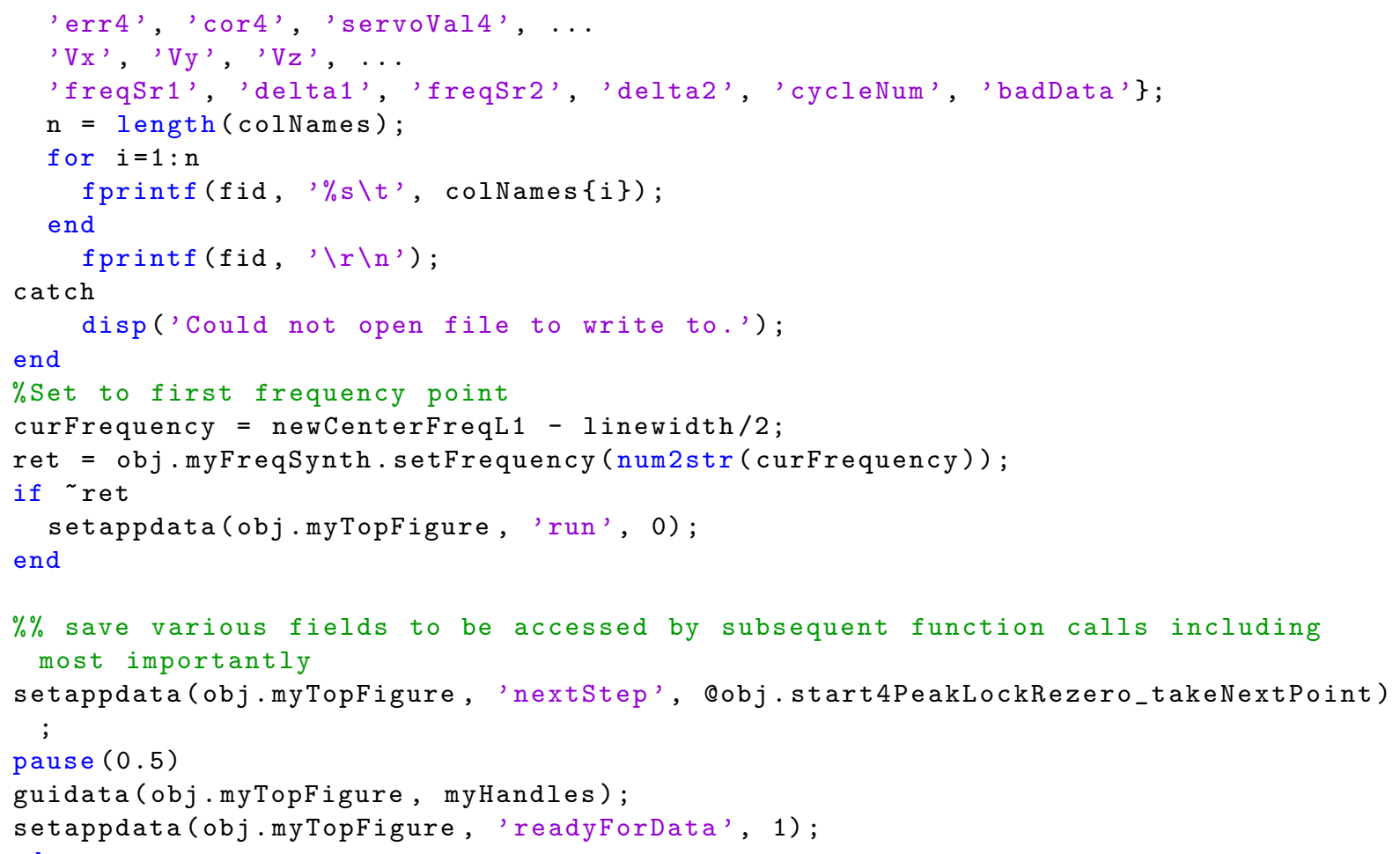

Figure 3.11: Code Snippet 10

As can be seen in this greatly simplified code above, only a few major pieces of code actually get executed when the user hits the start button. After initializing a few fields, two very important variables are declared, seqPlace and rezeroSeqPlace. As was mentioned in Sec. 3.1.1 the best method we found for synchronizing two clocks was having them share a "Cycle Number" that gave each individual experimental run a numerical ID. This ID not only allowed for easy data tabulation, but it also opened up the door to programmatically cycle through the behaviors of the locking protocol. In these programs this is accomplished by reading off the numerical ID (known as the cycle number) and finding its modulo with the number of different locking behaviors or how often some rare behavior is supposed to occur. For example, if a field rezeroing is set to occur every 81 cycles the program will simplify find the answer to cycle number $\% 81$ and compare the result with the recipe in lines 12 through 22 in Fig. 3.11. As can be read in those lines, as long as the answer is 9 or higher the program is told to go on about its business. In that case, to find where the program is in the sequence, it takes the cycle number and finds its modulo with 8. As will be seen soon, the action taken by the program will follow the protocol laid out in lines 4 
through 11. While much of the rest of this code is boilerplate for beginning a locking sequence, the most important line is 57 in Fig. 3.11. This places a hook in the program that will launch the correct function when data has been collected from the atoms. Designing a new sequence can be accomplished very simply then by clearly laying out what the order of the sequence is and any strange cyclical behaviors like rezeroing, making sure all the data one is interested will be correctly written down like in lines 28-48, and setting the correct hook for your new program. All the real meat of interfacing with hardware and taking and analyzing the data is encapsulated in each of the protocol's _takeNextPoint functions.

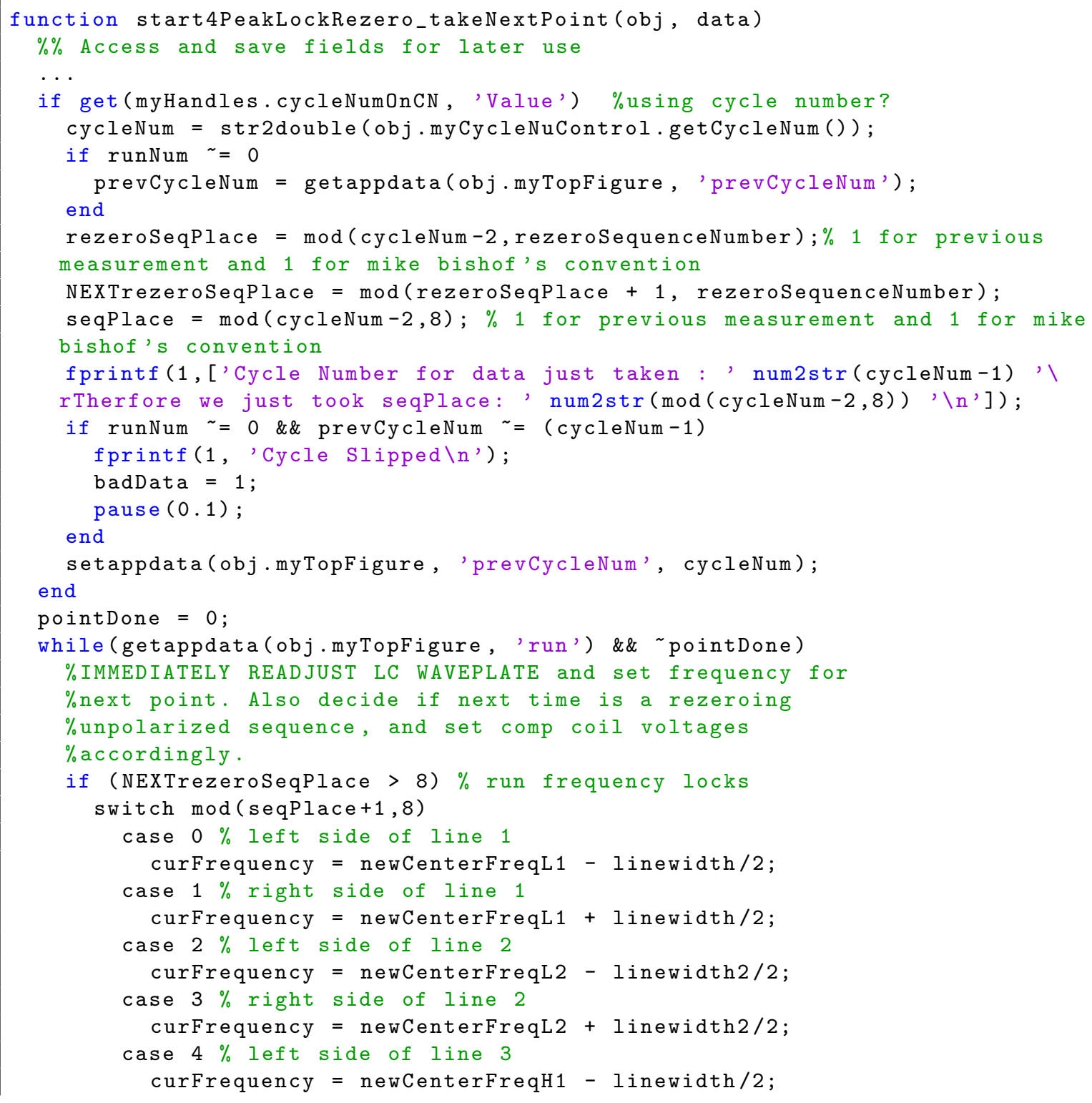




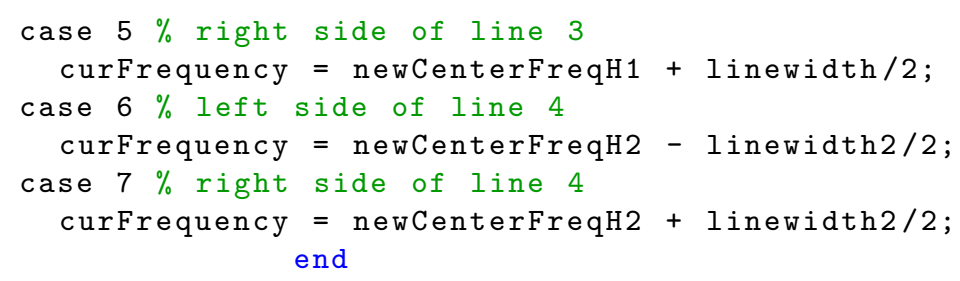

if get (myHandles.bouncelCwaveplate, 'Value') \&\& strcmp (get (myHandles.

openSeriallC, 'Enable'), 'off')

$\operatorname{disp}\left(\left[{ }^{\prime}: 2 ; c^{\prime}\right.\right.$ int $2 \operatorname{str}(\bmod (\operatorname{seqPlace}+1,8))$ '; d0; t80000']);

fprintf (obj.myLCuControl.mySerial , [':2; c' int2str (mod ( $\operatorname{seqPlace}+1,8))$ ' ;0; t $\left.80000^{\prime}\right]$ ) ;

end

else if (runNum > NEXTrezeroSeqPlace) \% i think this helps with bootstrapping.

switch mod (rezeroSeqPlace +1 , rezeroSequenceNumber)

case $0 \%$ Unpol, Ix $=$ IxO - Delta

curFrequency $=$ (newCenterFreqL1 + newCenterFreqH1) $/ 2$;

obj.changeAnalogVoltage (0, previousVoltages (1) - voltageStepSize);

case $1 \%$ Unpol, Ix $=$ Ixo

curFrequency = (newCenterFreqL1 + newCenterFreqH 1 ) $/ 2$;

obj.changeAnalogVoltage (0, previousVoltages (1) + voltageStepSize); $\%$ need

to add because of previous step in sequence.

case $2 \%$ Unpol, Ix $=$ IxO + Delta

curFrequency = (newCenterFreqL1 + newCenterFreqH1) $/ 2$;

obj.changeAnalogVoltage (0, previousVoltages (1) + voltageStepSize);

case $3 \%$ Unpol, Iy = Iy0 - Delta

curFrequency = (newCenterFreqL1 + newCenterFreqH1) $/ 2$;

obj.changeAnalogVoltage (1, previousVoltages (2) - voltageStepSize);

case $4 \%$ Unpol, Iy $=$ Iy0

curFrequency = (newCenterFreqL1 + newCenterFreqH 1 ) $/ 2$;

obj.changeAnalogVoltage (1, previousVoltages (2) + voltageStepSize); \%need

to add because of previous step in sequence.

case $5 \%$ Unpol, Iy $=$ Iy0 + Delta

curFrequency = (newCenterFreqL1 + newCenterFreqH 1 ) $/ 2$;

obj.changeAnalogVoltage (1, previousVoltages (2) + voltageStepSize);

case $6 \%$ Unpol, Iz = Iz0 - Delta

curFrequency $=$ (newCenterFreqL1 + newCenterFreqH1) $/ 2$;

obj.changeAnalogVoltage (2, previousVoltages(3) - voltageStepSize);

case $7 \%$ Unpol, Iz $=$ Iz0

curFrequency = (newCenterFreqL1 + newCenterFreqH1) $/ 2$;

obj.changeAnalogVoltage (2, previousVoltages (3) + voltageStepSize); $\%$ need

to add because of previous step in sequence.

case $8 \%$ Unpol, Iz $=$ IzO + Delta

curFrequency $=$ (newCenterFreqL1 + newCenterFreqH1) $/ 2$;

obj.changeAnalogVoltage (2, previousVoltages (3) + voltageStepSize);

end

if get (myHandles.bouncelCwaveplate, 'Value') \&\& strcmp (get (myHandles.

openSeriallC, 'Enable'), 'off')

disp(': $4 ; \mathrm{co}$; d0; t80000');

fprintf (obj.myLCuControl.mySerial, ':4;c0;d0;t80000'); end

else

curFrequency = newCenterFreqL1 - linewidth $/ 2$; end

end

ret $=o b j \cdot m y F r e q S y n t h \cdot$ setFrequency (num2str (curFrequency)); \%temporarily sets

clock laser frequency . . 
$89 \mid$ end

Figure 3.12: Code Snippet 11

The function in Fig. 3.12 has a few main objectives: first, it must setup the experiment for the next experimental run, and second, it must analyze and save the previously taken data. To accomplish this the program first queries an outside object for the cycle number. Following this, a series of switch and if statements steer the behavior of the program to correctly prepare the atoms and RF synthesizers. Above one can see two examples of this. The first works off of the seqPlace variable setting the right RF frequency for the next experimental run. The next bit of code similarly works off of the rezeroSeqPlace to correctly take the data for rezeroing the magnetic fields. Although I have removed the rest of the code, many similar decisions are made in the rest of the function call.

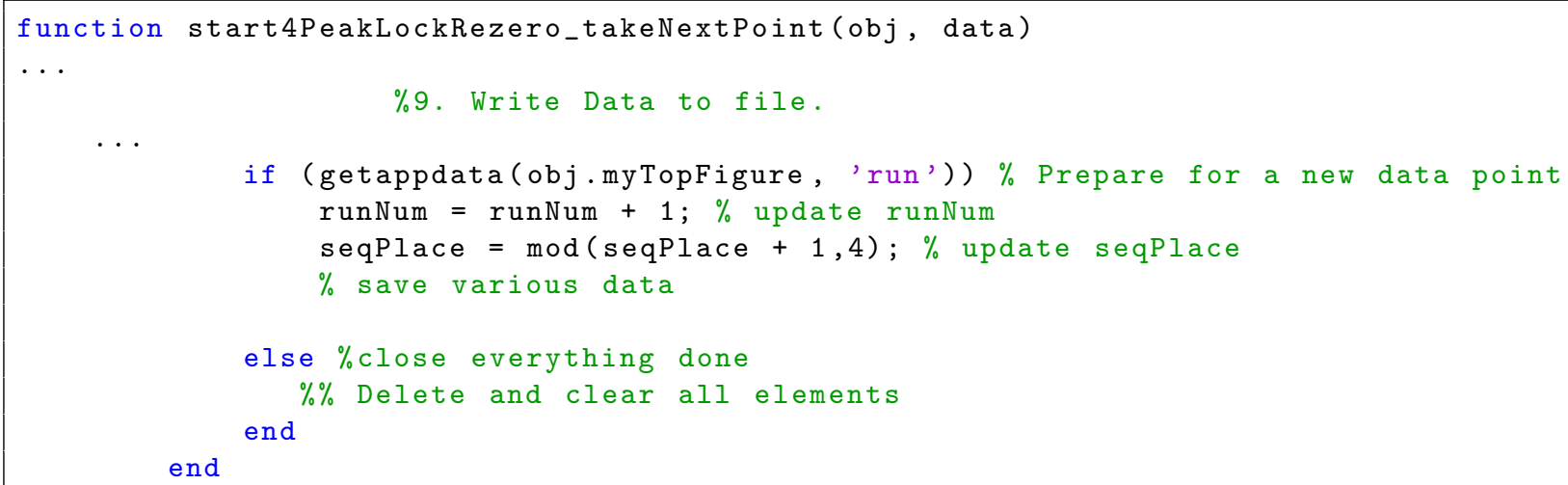

Figure 3.13: Code Snippet 12

As a reminder for future graduate students, the last bit of code to remember is to correctly deal with all variables and data that were created and plotted throughout running of the frequency locker. In fact, subsequent calls to start4PeakLockRezero_takeNextPoint only communicate to each other through storing variables in the appdata structure provided by Matlab. So all future sequences need to correctly remove all data and objects foisted onto this structure in place of line 11 of the code above or else running the locker might result in Matlab taking more and more of the control computer's RAM until the program crashes. 


\subsubsection{Arduinos: Simple, flexible Timing for Complicated Sequences}

To accomplish many of the tasks required to change the optical pumping polarization, turn on and off bias fields, and switch analong switches the FreqLocker program needs to be able to send out digital TTL signals on demand. The use of an arduino or any microcontroller makes this very simple. All that is needed is to create a common language for the arduino and the Matlab program to speak to each other. A careful reading of some of the code above will show that the program routinely writes data like :4; c0 to the object myLCuControl. This is a two part command that first tells the arduino which 'Mode' it is in (4) and second what cycle number is coming up (0). ${ }^{4}$

With this tool it's very easy to create constant TTLs that can be changed via the FreqLocker program.

But because this is a microcontroller, one is not limited to only creating DC values. In fact, one can feed TTL signals into the microcontroller and depending on the cycle number or 'Mode' change the behavior of the arduino's output to be an AND, or an OR, or any logical calculation of the TTL signals. As seen below this is easily done with macros.

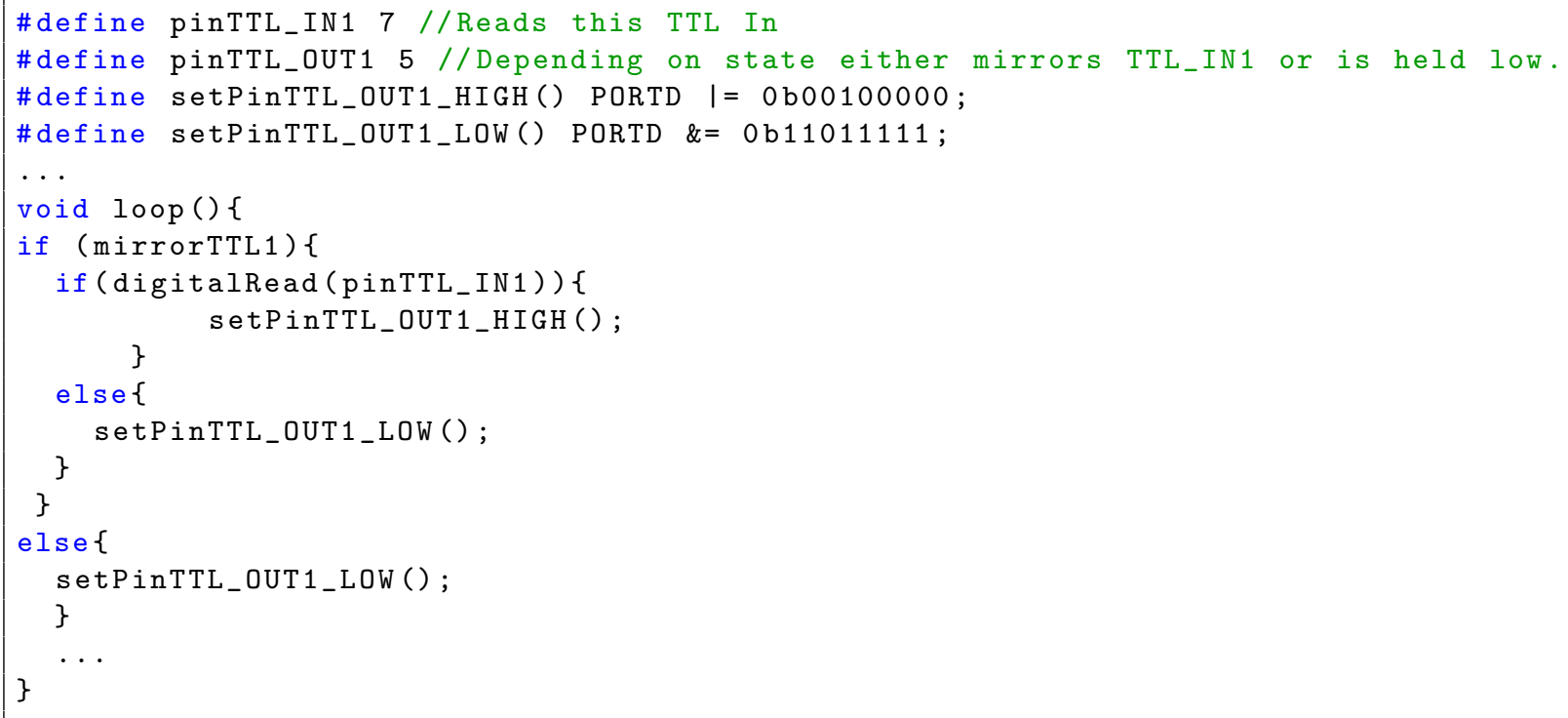

Figure 3.14: Code Snippet 13

\footnotetext{
${ }^{4}$ The rest of the message was built to house instructions for Ramsey TTL signals, however arduino timing jitter for slow clock speed arduinos may introduce noise into the atom signal.
} 
Inside loop, the behavior is governed by state variables that are set by parsing the messages from FreqLocker. Although this means that complicated frequency locker programs are essentially spread out over both Matlab and arduino files, the array of what is possible to do in these sequences is wildly expanded. 


\section{Chapter 4}

\section{Where in the world is ${ }^{1} \mathbf{S}_{0} \rightarrow{ }^{3} \mathbf{P}_{0}$}

2008 was a tumultuous year for America. With the subprime mortgage crisis leading the country into a recession, a pretty divisive presidential election, and ultracold fermion optical lattice clocks seeing a very clear atom-dependent collisional frequency shift, people were unsure about what the future would hold. Many obstacles stood in the way of optical lattice clocks. In some ways, these systems brought with their claims of high stability an unexplored territory of systematics that needed to be understood and characterized to incredible levels of precision. The first round of strontium systematic uncertainties just barely edged by the state-of-the-art cesium fountains,

reaching total systematic uncertainties of $1.5 \times 10^{-16}[1,63]$. To do better than this level of performance two things needed to occur. First, either considerable measurement time or the use of a much more stable local oscillator was necessary. And second, measurement techniques or fundamentally different clock designs were needed to address the three largest systematics: densitydependent shifts, AC Stark shifts, and BBR external temperature shifts.

A major obstacle to this work was the inability to take absolute measurements, as we were no longer looking for a factor of two improvement over current standards. This required us to innovate where the additional relative measurement precision, afforded to us by the use of the more stable laser, was not enough to achieve our goals [62]. The following two chapters are expanded versions of Ref. [22] made to fully explain all measurements, arguments, and statistical methods employed to reach our final systematic uncertainty of $6.4 \times 10^{-18}$. Where applicable, I will mention additional measurements or ideas for improvements to each of these systematic effects for future 
clock evaluations.

\subsection{Statistical Methods for Data Analysis}

For all systematic measurements, residual non-white noise introduces over scatter in the data. Thanks to the influence of precision measurement experiments to the statistical techniques employed in our strontium group at JILA, I believe we have taken a very conservative approach to quoting our results. Much of the data taken in this paper consists of a sequence of difference measurements taken between two oscillating experimental conditions. Of course there are many ways to find the difference between subsequent measurements. Imagine a sequence with frequency measurements $a$ and $b$ taken like so: $\left(a_{1}, b_{1}, a_{2}, b_{2}, a_{3}, b_{3}, \ldots\right)$. We could perform "forward differences" like $a_{2}-b_{1}$, or we could perform "backward differences" like $a_{1}-b_{1}$, or we could create a weighted difference between the two. In fact, there are a seemingly infinite number of choices. The creation of these correctly weighted sums of differences is known, at least in our group, as point-string analysis. In equation 4.1 one can read off exactly how to weight each of these frequency measurements to create ever longer strings [64].

$$
\delta \nu=\frac{1}{M-n+1} \frac{1}{2^{n-2}} \sum_{l=1}^{M-n+1} \sum_{m=1}^{n}(-1)^{l+m-1} \frac{(n-1) !}{(n-m) !(m-1) !} \nu_{l+m-1}
$$

Where $M$ is the total number of frequency points, $n$ is the point-string length, and $\nu$ are the frequency measurements. For example for the third point string in the series above with $n=3$, $\delta \nu(l=3)=-\nu_{3}+2 \nu_{4}-\nu_{5}$.

It should be noted that due to the overlapping nature of this data analysis method, each point string is not statistically independent. A correction factor according to equation 4.2 must

be included when looking at the statistical significance of results produced via the point-string method. Any standard deviations calculated using point strings should be multiplied by the factor noted in equation 4.2. An added benefit for this method is that for increasingly larger $n$, the data becomes immune to polynomial-like correlations between points. For example, starting with $n=3$ 
the data is immune to linear drifts in the data [64].

$$
f_{\text {correction to std err }}=\frac{2^{n-1}}{\sqrt{\sum_{m=1}^{n}\left(\frac{(n-1) !}{(n-m) !(m-1) !}\right)^{2}}}
$$

Data analysis on many of the experiments begins by examining the simple means, standard errors, and Allan deviation of these point-string quantities.

To quote a final number for a measurement, however, is a little bit more involved. This process begins by binning the data into small chunks. The bin size can and should vary greatly, again to examine if the data is behaving in a consistent manner for quoting a statistically significant result. For all bins, a mean and standard error, including corrections for point string and student-t effects (undersampling of the standard deviation), are calculated. This allows for the calculation of the reduced chi-squared, $\chi_{\nu}^{2}$. As is the case for almost all our data, residual $1 / f$ noise pushes the value of $\chi_{\nu}^{2}$ above 1 . In instances where this occurs a simple weighted mean of the bins would seem to underestimate the error with which the final result is known. Instead, the bins' errors are inflated by the calculated $\sqrt{\chi_{\nu}^{2}}$, thereby making the error on the final quantity larger and more statistically relevant. However, minor changes in bin size, although they should not effect the mean, can cause fairly large fluctuations in the final quoted error. To combat this, the analysis is performed for all bin sizes ranging from 35 to 75 points per bin where undersampling is less of an issue and the distribution of deviations is flat, and the error quoted is the simple mean of all bin sizes' calculated error. This conservative approach is applied to all measurements in this thesis unless otherwise noted.

\subsection{The Density Shift}

The initial observations of cold collisional shifts in a Sr OLC showed only a very small signature of shifts at a few parts in $10^{-16}[1]$. Reaching accuracies below $10^{-17}$ would require precise knowledge and understanding of these shifts. While work proceeded on finding novel suppression techniques of these shifts, progress on a complete understanding of these shifts was slowed due 
to the complexity of modeling Rabi shifts [64]. Subsequent work based on Ramsey spectroscopy for both $\mathrm{Sr}$ and $\mathrm{Yb}$, however, finally explained a completely self-consistent picture of $2 \mathrm{D}$ p-wave collisions occurring inside the lattice pancakes $[65,34,66]$. Regardless of the s- or p-wave nature of the shift ${ }^{1}$, as a clockmaker chasing the always on the move accuracy target, we needed to find an easy way to suppress collisions.

While significant advances had been made by working at the so-called "zero crossing" of the density shift in Ramsey clock operation, the complexity of Ramsey operation and the stability of the zero-crossing to other parameters had not yet been explored [65, 34]. Instead, SrII focused on the reason why no other strontium groups had yet to report statistically significant density shifts. Regardless of the lower precision of some other competing experiments, the explanation for the disagreement was the much more mundane fact that referring to the shift as being caused by some number of atoms is incomplete [54]. The number of atoms is not as important as the number of collisions that the atoms can undergo, which is of course more of a function of density and lattice occupation. Not only do the loading dynamics into one's trap matter but also the trap frequencies and the atoms' temperature. The SrI system was seeing large density shifts because they were loading a dense sample into a very tight trap with densities at the mid $10^{11} \mathrm{~cm}^{-3}$.

The simplest way for SrII to decrease the effect of the density shift was to operate at lower densities around a few $10^{8} \mathrm{~cm}^{-3}$. This lowers the magnitude of the density shift by almost a factor of 30 even at SrI's lowest attainable densities. This was accomplished via the use of the build up cavity mentioned in Chapter 2 which traps the atoms in a significantly wider beam waist for moderate input trapping powers. A much higher power laser could also accomplish this task without a cavity. An added benefit of the larger trap volume was of course the ability to better overlap the lattice with the cold red MOT, loading more atoms. As has been seen both in explanations of the density shift and been borne out of experimental data the density shift is, at our current precision, linear in atom number. Therefore, the ability to run the system with upwards of 100,000 spin-polarized atoms allowed us to measure a significantly larger shift and scale down to the atom number for our

\footnotetext{
${ }^{1}$ In $2 \mathrm{D}$, p-wave collisions are temperature independent which is plus for making $1 \mathrm{D}$ lattice clocks.
} 
clock operation with exceedingly high precision. Measurements like this are showcased in Figure 4.1 where the lever arm has allowed us to measure the shift per atom very precisely very quickly.

However, the measurements reported in Ref. [62] are taken at deeper lattice depths than we can accurately run the clock at. Running the clock at lower lattice depths reduces the atom density further, decreasing the shift, in a very nonlinear manner. In fact, if we were somehow to run the lattice at $5000 \mathrm{E}_{r}$, even an incredibly modest number of atoms could cause frequency shifts on the order of $10^{-14}$. So as not to rely on pure density scaling arguments we proceeded to take density shift measurements as close to our operating lattice depth as possible. Extrapolation of the density shift due to changes in the lattice trapping potential was used only when trap frequencies had changed by less than 10\%. Additional error from this extrapolation is included in the final quoted uncertainty, although it did not significantly change the final error. For a SrII lattice with depth of $87 \mathrm{E}_{r}$, the final shift for $\sim 2000$ atoms was $4.7(6) \times 10^{-18}$. This corresponds to approximately 1.8 atoms per pancake, with axial temperatures near $2 \mu \mathrm{K}$ and radial temperatures near $3 \mu \mathrm{K}^{2}$ Without careful measurements of the cloud temperatures at these low density limits of SrII, uncertainty in the temperatures restricts the accuracy of our average density in an occupied lattice site to $1.0(0.7) \times 10^{9} \mathrm{~cm}^{-3}[54]$.

Future experiments should find a healthy balance between SrI and SrII's density shifts. While the use of a buildup cavity to ensure low densities is cumbersome, using higher power lasers will allow one to forgo the many complications of using a buildup cavity. Furthermore, the use of colder atomic samples and vertical lattices will allow atoms to be loaded into even less dense lattices further reducing the density shift. Active cancellation via feedback operation at the zero-crossing of the Ramsey density shift for low density lattices is another avenue worth exploring in the future as well [65].

\footnotetext{
${ }^{2}$ Recall that in $2 \mathrm{D}$ p-wave collisions are to first-order temperature insensitive.
} 


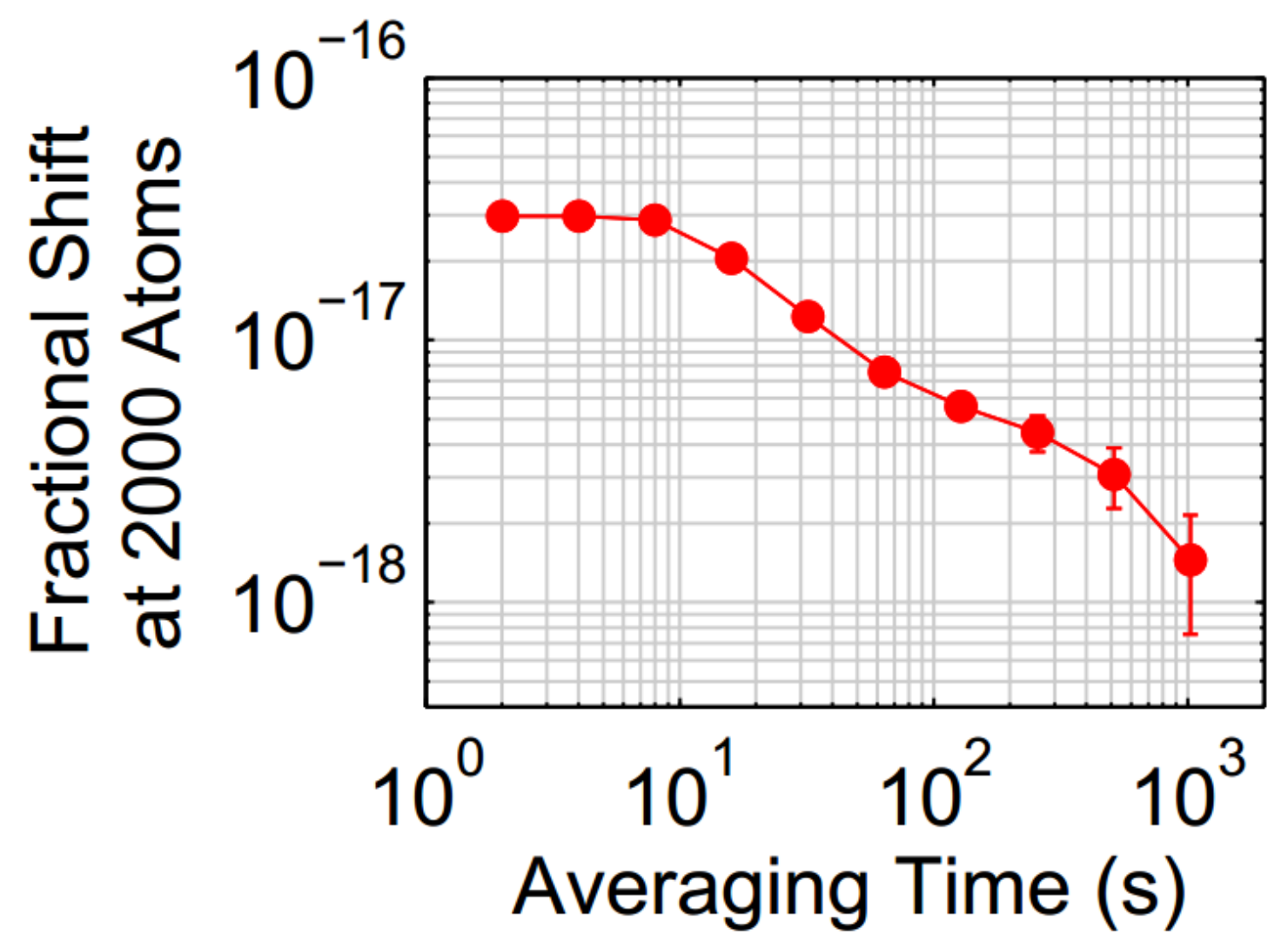

Figure 4.1: An Allan deviation of a large lever arm density shift measurement, reproduced from ref. [62]. Disregarding the modulation of the atom number, this data was taken at a higher than normal clock operating density. The shift per count has been scaled to reflect clock operation uncertainty for 2000 atoms. Other measurements have shown even higher stability, and make it clear that the density shift, although an annoyance, should not be considered a problem for future higher accuracy clocks. 


\subsection{The Zeeman Shifts}

Alkaline earth elements' paired electrons have an inherent immunity towards magnetic field related shifts. For instance, the boson, ${ }^{88} \mathrm{Sr}$, has no nuclear spin and does not exhibit any magnetic field tuning of the ground state scattering length. The fermion's tiny nuclear moment couples very weakly with the field, and the differential g-factor between the ${ }^{1} \mathrm{~S}_{0}$ and the ${ }^{3} \mathrm{P}_{0}$ leads to a small shift coefficient of $108 m_{F} \mathrm{~Hz} /$ Gauss between $\pi$ transitions. Of course, even this small shift for 1 Gauss of magnetic field is almost six orders of magnitude larger than we are trying to control our clock to. Luckily, how to control the first and second order shifts Zeeman effects is well understood.

\subsubsection{The First-Order Zeeman Shift}

The first-order Zeeman shift is canceled by alternately interrogating opposite nuclear spin stretched states. By sending the ${ }^{1} \mathrm{~S}_{0}$ to ${ }^{3} \mathrm{P}_{1}(F=9 / 2)$ optical pumping laser through a liquid crystal waveplate, one can easily switch between polarizing the atoms in the $m_{F}=+9 / 2$ or the $m_{F}=-9 / 2$. After each line center acquisition of the clock laser, the atom polarization is swapped. The quoted Sr frequency is the running average between the two previous measurements, canceling out any linear magnetic field effects in $m_{F}$. Key to the efficacy of this scheme is that changes in the magnetic field over two line center acquisitions are either negligible or averaged away.

The servo controlling the bias field splitting between the nuclear spin states was unfortunately not perfect, and would slowly drift over long timescales. Not only did this cause some extra uncorrelated noise between SrI and SrII in comparisons, but it also made us worried that the first order Zeeman shift was not being perfectly canceled by the alternating interrogation scheme. If an overall breathing mode of the field persisted throughout the measurements one could imagine this causing a shift. A residual first-order Zeeman shift is calculated by examining the overall drift of the magnetic field splitting between the two stretched nuclear spin states, and extrapolating the inherent shift this drift would induce. If a biased derivative existed in the splitting data it could be extrapolated to cause a shift. Luckily, no statistically significant drift existed in the data. 
Table 4.1: Second order Zeeman shift coefficients and their excellent agreement around the world.

\begin{tabular}{llll}
\hline Location & Year & Coefficient $\left(\mathrm{Hz}^{-1}\right)$ & Uncertainty $\left(\mathrm{Hz}^{-1}\right)$ \\
\hline JILA SrI & $2008[63]$ & 2.58 & 0.35 \\
PTB & $2011[67]$ & 2.43 & 0.03 \\
LNS-SYRTE & $2011[35]$ & 2.46 & 0.02 \\
JILA SrII & $2014[22]$ & 2.48 & 0.02 \\
\hline
\end{tabular}

Furthermore, a recent update to this coil servo has suppressed the field drift.

\subsubsection{The Second-Order Zeeman Shift}

The second-order Zeeman shift, caused by the presence of an applied bias and a residual magnetic field during the clock interrogation sequence, is subtracted off point by point. The determination of the second-order Zeeman shift coefficient is made via a fast modulation experiment whereby four digital locks are modulated in sequence: two locks at a high magnetic field, one for a measurement of each stretched spin component, and two locks at a low magnetic field. This experiment was performed for stretched states splitting ranging from $300 \mathrm{~Hz}$ to 1,200 Hz. Because the shift is quadratic in nature and we end up running the clock at the low end of this measurement we gain a large lever arm for determining the second-order shift coefficient.

To fit this data, a quadratic function, $c S^{2}$, was used. Here $c$ was found to be $-0.248(2) \times$ $10^{-6} \mathrm{~Hz}^{-1}$, where $\mathrm{S}$ is the measured stretched state splitting in Hz. Extra care was taken to ensure that modulation of the applied bias field did not cause any rotation of the polarization axis, which would induce an unwanted differential lattice tensor shift. Table 4.1 compares our measurement of this coefficient with previously reported measurements, showing excellent agreement. By fitting this coefficient in terms of the frequency splitting measured rather than in SI units of field, we bypass our imperfect knowledge of the $\delta g$ factor $^{3}$. The second-order Zeeman shift uncertainty of $1.2 \times 10^{-18}$ is quoted for a $500-\mathrm{Hz}$ splitting between the $m_{F}= \pm 9 / 2$ stretched states, which allows for a relatively strong bias magnetic field of about half a gauss $(50 \mu \mathrm{T})$ to be applied to the atoms

\footnotetext{
${ }^{3}$ Although, we introduce other errors as described in Section 4.4.2
} 
during clock operation. Future measurements with the new coil servo have already been performed at higher field splittings further reducing the uncertainty in the second order Zeeman coefficient and its shift of the clock transition [68].

\subsection{The AC Stark Shifts}

The basis of many of the state-of-the-art experiments dealing with ultracold atoms includes their trapping or storage inside a far detuned optical trap [69]. Whether this trap is formed at the intersection between different beams that are either phase coherent or not simply changes the geometry of the trap. Fundamental to the idea of trapping neutral atoms is that the electron cloud around the atom becomes polarized, intrinsically deforming the various wavefunctions in the atoms. This changes the energy splittings between various levels, and would on the surface at least discount the very possibility of using neutral atoms as atomic clocks. The saving grace of using a transition inside a multi-level atom as an atomic clock is of course the fact that the polarizabilities as a function of frequency for both the ground and excited states goes through many resonances and there are gentle crossings where these polarizabilities are equal [32]. These "magic wavelength" trapping points ideally leave no differential trapping shift during clock operation.

In a real system we can only hope to asymptotically approach this ideal limit. The ability to which we can measure where the magic wavelength trap is located is limited by the precision level of the measured residual trapping shift. Furthermore, higher order shifts related to either

the hyperpolarizability (essentially two-photon coupling) [70] or non-E1 couplings [71] are hard to measure due to their weak nature. In this section we will examine our most recent exploration into all of these shifts, and cross check with work performed around the world also regarding these shifts in strontium OLCs.

\subsubsection{The Scalar and Tensor Shifts}

To ease comparison with other published papers on lattice shifts I will use the notation found in Ref. [35]. In this section we will discuss the shift coming from scalar and tensor components of 
the polarizability,

$$
\Delta \nu^{E 1}=\left(\Delta \kappa^{s}+\Delta \kappa^{t} \beta\right) U_{0}
$$

where $\beta=\left(3\left|\vec{\varepsilon} \cdot \vec{e}_{B}\right|^{2}-1\right)\left\{3 m_{F}^{2}-F(F+1)\right\}$; the trap depth, $U_{0}$, is expressed in units of lattice photon recoil, $E_{r} ; F$ refers to the total spin of the atom, $m_{F}$, refers to the projection of this spin onto the quantization axis; and $\vec{\varepsilon}$ and $\vec{e}_{B}$ are the unit vectors of the lattice polarization and the magnetic field quantization axis, respectively. Varying the angle between the quantization axis and the lattice polarization allows one to separate out the different contributions of the scalar and tensor shifts. The SYRTE group did just that, and reported $\Delta \kappa^{s}=0$ when trapping with a laser tuned to $\nu_{\text {trap }}=368554693(5) \mathrm{MHz}$ and $\Delta \kappa^{t}=-0.0577(23) \mathrm{mHz} / E_{r}$ [35]. While it is useful to know these numbers, the actual operation of a clock does not really require one to separate these two effects which are linear in lattice depth.

Instead, we treat both effects as if they came from the same source. We are able to do this because we make the magnetic bias field direction to be the same as the lattice polarization, and always run our clock on the $m_{F}= \pm F$ stretched states. However, due to the unfortunate proximity of our ion pump magnet to the MOT coils, a slow DC magnetic field wander is observed as elements of the apparatus slowly change their magnetization on the tens of minutes time scale. Active control of the magnetic field was implemented to combat this. We use the atoms themselves as a collocated magnetometer for the clock. Every two minutes during the clock operation, the computer-based frequency locking program pauses to interrogate unpolarized atomic samples under a zero applied magnetic field. A drift in the background magnetic field away from zero results in a reduced excitation signal for the peak of an unpolarized excitation, because all ten nuclear spin states will experience different Zeeman shifts. As can be seen in figure 4.2, the magnetometer-based feedback loop not only keeps the field direction constant throughout the clock operation, but also automatically nulls the background field without an operators intervention.

Once the clock has entered into the servo routine, it takes three peak excitation measurements for each pair of magnetic field compensation coils (along three orthogonal spatial directions) at 

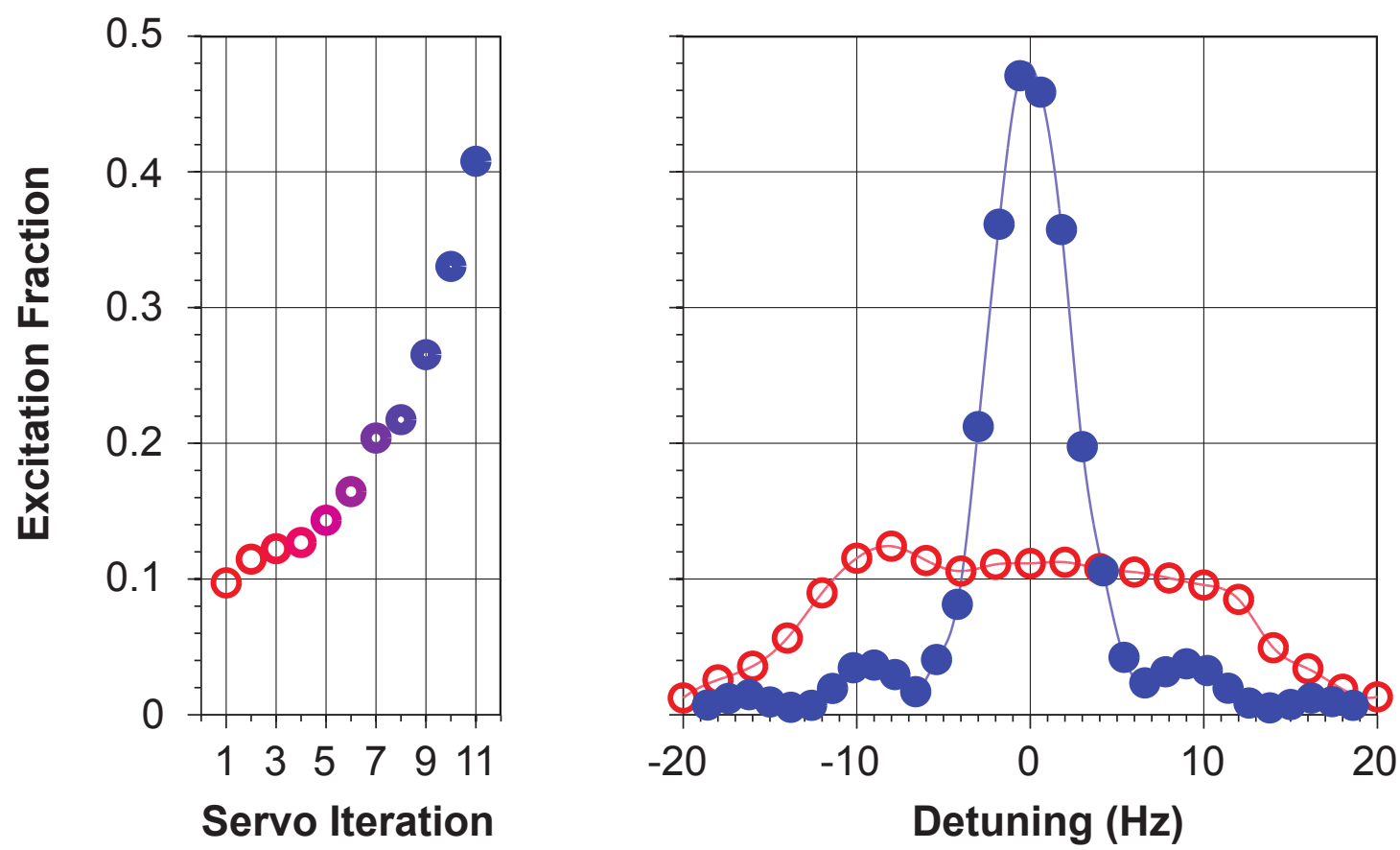

Figure 4.2: Using the atomic cloud as a collocated magnetometer, a residual non-zero magnetic field is inferred via the peak excitation of an unpolarized Rabi lineshape. The left panel shows the servo action of zeroing the residual magnetic field. The slow action is due to the implementation of a simple FIR mean filter on the servo's output, trading off stability for speed. The right panel shows a clock transition lineshape for an unstabilized magnetic field (red circles) and an improved lineshape under the stabilized magnetic field (blue filled circles). Reproduced from ref. [22]. 
different currents $\left(I_{0}-\Delta I, I_{0}, I_{0}+\Delta I\right)$ where $I_{0}$ is the current of the previous iteration and $\Delta I$ is a small trial step. In situations where the residual field is far from zero, the software steps the current by a fixed amount in the direction indicated by the increasing excitation. When the field is near zero, a parabola is fit between the three measurements and the current is stepped to the fitted value filtered via a low pass finite impulse response filter. On stopping the servo routine and measuring the unpolarized line, the residual magnetic field wander was measured to be less than $0.3 \mu \mathrm{T}$.

I would like to note that the choice of the parallel orientation between the quantization axis and the lattice polarization is very beneficial for clock operation. While the $\beta$ term in equation 4.3 can be set to zero at the "magic" angle, this is not the optimal orientation to run a clock! By setting the angle between the two axes to be 0 , our clock is to first order linearly insensitive to variations of this angle. While this cannot be said for the magic angle. Precision alignment of these two axes is performed via iterative minimization of the appearance of residual $\sigma^{+(-)}$transitions from the stretched ${ }^{1} \mathrm{~S}_{0} m_{F}=-(+)^{9} / 2$ states to the ${ }^{3} \mathrm{P}_{0} m_{F}=-(+)^{7} / 2$. Figure 4.3 shows an iteration of the minimization process where the signal for setting each coil's current is clearly visible. Coupled with the zero field holding magnetic field servo, this greatly relieves the burden on how exactly to set your quantization axis parallel to the lattice polarization. This was especially necessary in our case due to the somewhat random birefringence in our vacuum viewports which caused us to choose a polarization mode set by the optics themselves.

To successfully measure and extrapolate the shift with respect to trap depth, a method for determining the trap depth at each measurement point was necessary. Assuming that the power detecting photodiode had a perfect relationship to the trap frequency would ignore issues like gravitational sag thereby introducing needless systematics in the measurement. Instead, trap frequency measurements were performed at each measured AC Stark point via a high-resolution sideband scan. As explained in ref. [72], radial motion only brings the sidebands closer to the carrier, so the true longitudinal sideband frequency is found by looking at the farthest edge of the blue sideband. This edge corresponds to the contributions of atoms that are distributed at the 


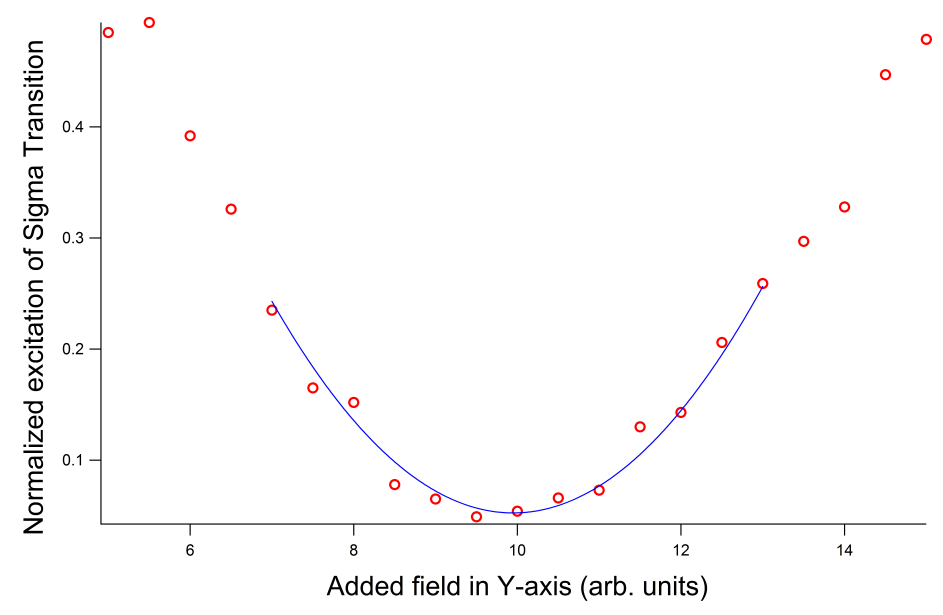

Figure 4.3: To more precisely align the field of the clock laser, lattice polarization, and quantization axis we iteratively minimize the peak of any visible transitions driven by circularly polarized light. Depicted above is one of the minimization iterations of the Y-coil. The clock laser intensity is run 4 or so orders of magnitude higher than during clock operation. Further iterations lose track of the sigma transition as it cannot be seen below the noise. The blue line is a fit to the data used to pick a value for the Y-coil before moving on to other coils. 
center of the Gaussian profile for the lattice beam. A scan of the carrier was taken simultaneously with these high-resolution sideband scans, and a fit to the carrier was used to determine the exact fourier-limited linewidth. The hard, outer, vertical edge of the sideband is fit with a straight line and its frequency-intercept (x-intercept) is noted. This intercept can then be related to the intercept of a Lorentzian's tangent line at its inflection point. Using both the intercept and linewidth, an approximate position for the center of the lorentzian can be found. Once the longitudinal trap frequency was determined, the trap depth could be extracted using the procedures outlined in ref. $[72]$.

Alternating between four atomic servos, with the magnetic field control activated, we measured differential shifts between a variety of high and low lattice depths from 87Er to 300Er for the SrII apparatus. To minimize systematic uncertainties caused by differential atomic density shifts, both high and low lattice depths were operated with an absolute density shift below $1 \times 10^{-17}$. As was mentioned previously, atomic interaction shifts follow a power-law behavior in trap frequency, and must be taken into account especially at very high lattice depths. Atom numbers were chosen to provide similar density shifts for both high and low lattice depths allowing for common-mode cancellation. Furthermore, individual measurements for particular values of lattice depth difference were performed at $1 \times 10^{-17}$ statistical uncertainty. Many measurements at different lattice depths were needed to achieve the low uncertainty reported here (see Fig. 4.4). Combined with other analysis to determine higher order shifts, discussed in a subsequent section, this data represents one of the most precise measurements of the AC stark shift yet.

Although many of these measurements took place many $\mathrm{MHz}$ away from the scalar+tensor magic wavelength, the system was briefly used to pin-point where this zero crossing wavelength existed. Even though this was not a full systematic study, I believe this number should be used to at least guide further study of this colloquial magic wavelength, $\nu^{*}=368.554485 \mathrm{THz}$. Future experiments should focus on a few different systemwide optimizations that would allow for a more robust AC stark shift and a lower uncertainty. To begin with, our highest lattice depths were limited by laser power due to a faulty $10 \mathrm{~W}$ Verdi pumping the Ti:Sapphire laser used to generate 


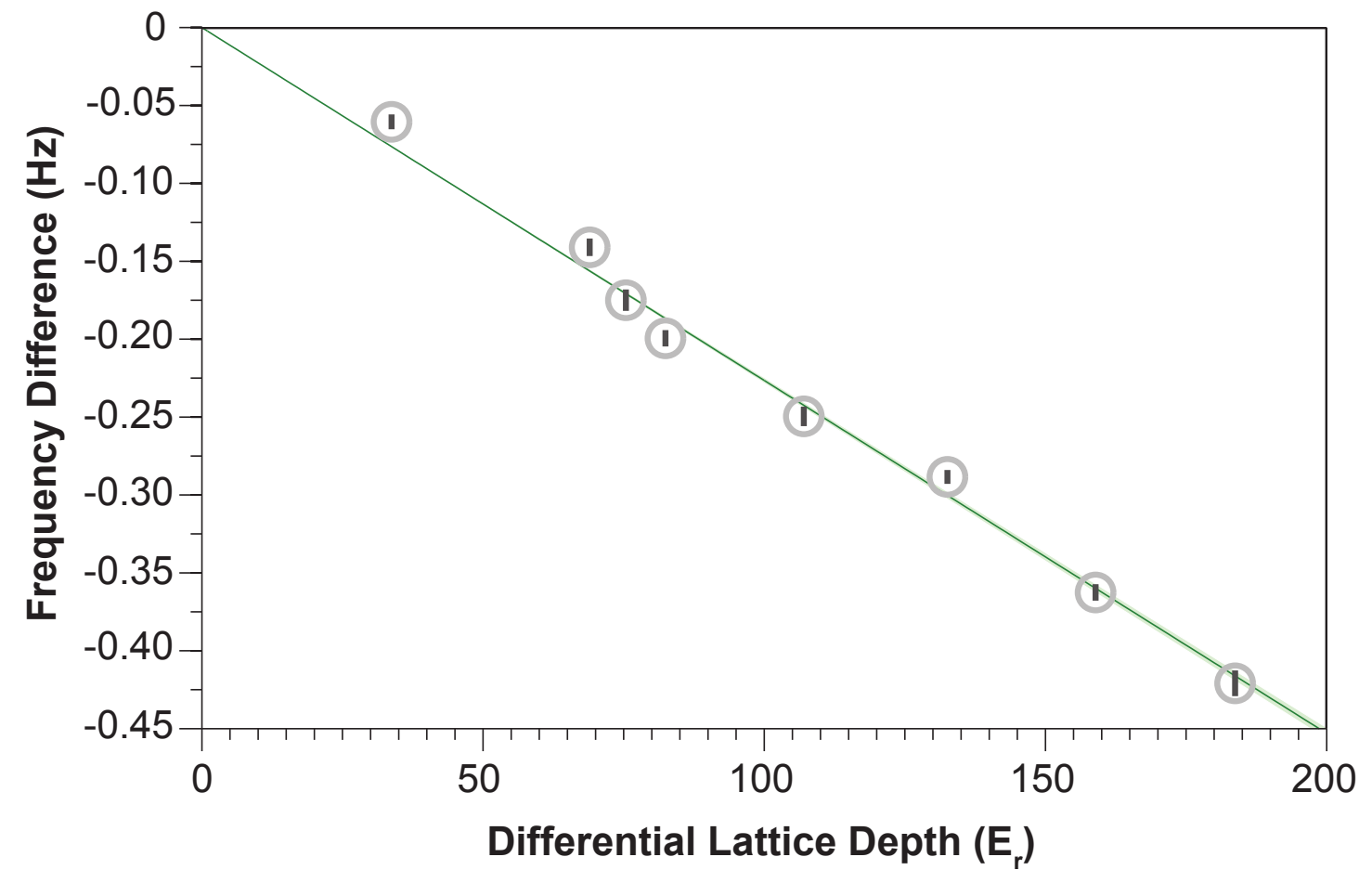

Figure 4.4: To determine the lattice AC Stark effect accurately, a variety of lattice depths were used. This effect is depicted as a function of the differential lattice depth with binning chosen for figure clarity (average bin size $68 \mathrm{~min}$, corresponding to an average of 1,600 points). Given the results of the F-test, the best fit is a linear model. Reproduced from ref. [22]. 
the lattice light. A better pump laser and impedance matching of the cavity mirrors would greatly raise the attainable power. Second, our low lattice depths were limited by the need to trap radially against gravity. Vertical lattices are much better suited to creating more accurate clocks. Finally, if magnetic field direction sensitivity ever becomes a problem again the use of the $m_{F}= \pm 5 / 2$ states will decrease the tensor shift magnitude by a factor of 6 . I believe that many of these suggestions will become necessities as clocks push below $10^{-18}$.

\subsubsection{The Vector Shift}

The vector Stark shift manifests itself as a fictitious magnetic field. Borrowing notation from Ref. [35] again,

$$
\Delta \nu^{E 1}=\left(\Delta \kappa^{v} m_{F} \xi \vec{e}_{k} \cdot \vec{e}_{B}\right) U_{0}
$$

where $\xi \vec{e}_{k}$ is the degree of circularity in the lattice. Because the shift is odd under the projection of the nuclear spin, the shift is cancelled by alternately measuring opposite nuclear spin stretched states just like the first order Zeeman effect. Previous measurements with the lattice, before the precision alignment of both the lattice polarization and the magnetic field direction, were shown to have a lattice vector shift on each state of a few tens of $\mathrm{mHz}$. With the addition of an extra polarizer that both the clock laser and lattice copropogated through, a spectroscopic alignment of the quantizaiton axis was performed following this vector shift discovery. A worst case extra splitting of $100 \mathrm{mHz}$ was included in the error budget. This extra splitting would introduce a systematic error to the second order Zeeman shift correction. Calculations, however, show that this residual vector shift is far below $10^{-19}$.

\subsubsection{The Higher-Order Shifts}

Higher order shifts, if they were statistically significant, should have been seen in the data in figure 4.4. Model fits for both a hyperpolarizability, quadratic in lattice depth, and an E2/M1 contribution, proportional to the square root of the lattice depth, revealed no statistically significant contribution, based on a Fisher test with a $1 \sigma$ threshold. To be clear, without precise knowledge 
of the magnitude of either of these two terms, a Fisher test for the inclusion of an additional term in the fitting equation was used. This test, as outlined in ref. [73] section Test of Additional Term, compares whether the decrease in the $\chi_{\nu}^{2}$ of the fit to the data is consistent with white noise or not.

Even at this measurement precision, it's possible that the small magnitude of these shifts allowed some measure of their contributions to be included in the linear fit. Our fitted hyperpolarizability, $0.48(47) \mu \mathrm{Hz} / E_{r}^{2}$, is not inconsistent with previously reported coefficients of $0.45(10) \mu \mathrm{Hz} / E_{r}^{2}$ [27]. However, our data, as shown by the Fisher test, supports a linear fit only. We thus list only a single overall systematic uncertainty for the lattice AC Stark shift, treating the entire data set in the most statistically consistent manner. We note that using a prior reported hyperpolarizability coefficient, our overall AC Stark uncertainty would change only slightly from $3.7 \times 10^{-18}$ to $4.1 \times 10^{-18}$. In the previous measurement by the SYRTE group, in order to gain a very large lever arm for the measurement of the hyperpolarizability, a very high $\left(5000 E_{r}\right)$ lattice depth was used, but the atomic density effects in such tight traps were seemingly not considered. Simple scaling of the density shift would imply enormous frequency shifts even for small numbers of atoms. In summary, although this data is not inconsistent with the hyperpolarizability measurements previously reported, this work is not an independent verification of their measurement. Future work, involving higher stability clock lasers, will no doubt observe and catalogue these more exotic shifts.

\subsection{The DC Stark Shift}

Strontium is somewhat cursed with a fairly large differential polarizability between the ${ }^{1} \mathrm{~S}_{0}$ and the ${ }^{3} \mathrm{P}_{0}$ states. So the presence of any external DC field, or low frequency radiation that gets rectified by the atoms, will cause a significant shift. This is what leads to strontium's higher sensitivity to blackbody radiation than other alkaline earth atoms. In the past, incomplete knowledge of the differential polarizability was also a leading contributer to the systematic uncertainty. However research done at PTB, where precise knowledge of an applied electric field was correlated with frequency shifts, allowed full determination of the DC differential polarizability, $\Delta \alpha=4.07873(11) \times 10^{-39} \mathrm{Cm}^{2} / \mathrm{V}[74]$. This measurement had an enormous influence on the outcome 
of this thesis, and its importance to the Sr OLC community cannot be overstated.

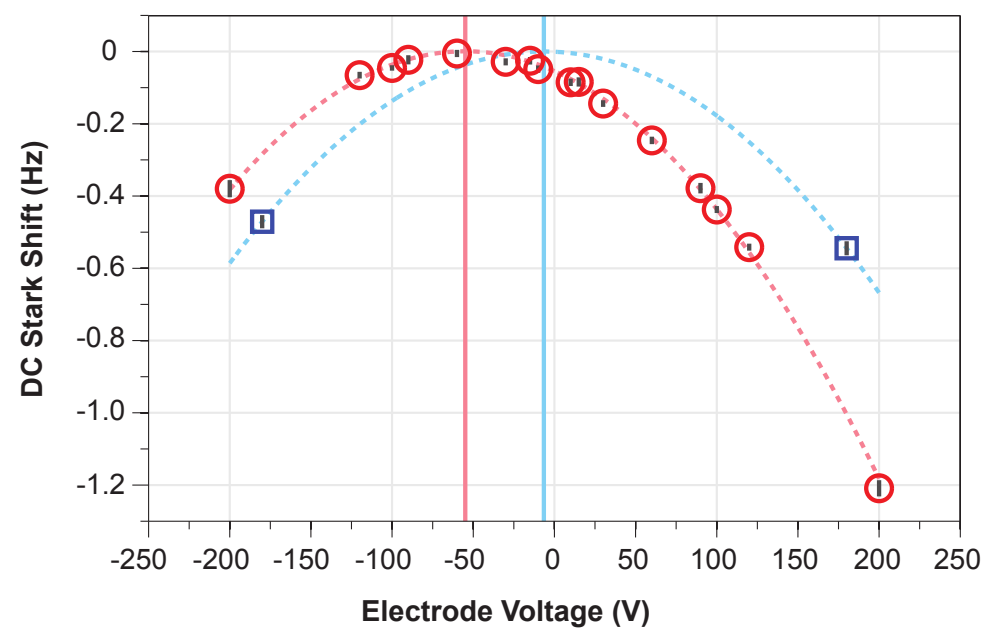

Figure 4.5: Measurements of DC electric-field-induced Stark shift show a quadratic behaviour. The red circles show that a residual shift due to the stray DC field was $-1.3 \times 10^{-16}$. The blue squares show a greatly reduced shift after purging the vacuum chamber with $\mathrm{N}_{2}$ gas. Dashed lines show a quadratic fit to the data. Solid vertical lines show the locations of a zero net electric field. Tracing out the quadratic form of the shift is not strictly necessary for measuring the shift. Reproduced from ref. [22]

Although we expected no shift, we decided to see if there were any stray DC electric fields in our system. We were worried that patch charges could be immobilized in the vacuum chambers fused silica viewports [55]. A pair of disk-shaped quadrant electrodes was placed near the two largest viewports in the system (separated along the vertical direction) and shifts were recorded as the electrode polarity was switched or grounded. Differences between the frequency shifts induced by oppositely charged electrodes indicate the presence of stray background electric fields, as shown in figure 4.5. To be clear, to successfully observe a residual DC shift with only one clock, without tracing out the quadratic behavior of the shift, three repeated measurements need to be performed. First, one must apply a field in the (arbitrary) positive direction: $\nu_{\uparrow}=-\frac{1}{2} \alpha\left(\vec{E}_{\mathrm{bg}}+\vec{E}_{\text {applied }}\right)^{2}+$ $\nu_{\text {laser }}$. Second, one must measure the frequency of the laser with the electrodes grounded: $\nu_{\text {gnd }}=$ $-\frac{1}{2} \alpha\left(\vec{E}_{\mathrm{bg}}\right)^{2}+\nu_{\text {laser }}$. Third, one measures the effect of the applied field in the (arbitrary) negative direction: $\nu_{\downarrow}=-\frac{1}{2} \alpha\left(\vec{E}_{\mathrm{bg}}-\vec{E}_{\text {applied }}\right)^{2}+\nu_{\text {laser }}$. Combining and manipulating these equations results 
in a measured shift as seen below.

$$
\delta \nu_{\mathrm{bg}}=\frac{\left(\nu_{\uparrow}-\nu_{\downarrow}\right)^{2}}{8\left(\nu_{\uparrow}-\nu_{\text {gnd }}+\nu_{\downarrow}-\nu_{\text {gnd }}\right)}
$$

On first measuring the DC Stark effect on SrII, a residual, stable $-1.3 \times 10^{-16}$ shift was discovered. However, when the vacuum chamber was filled with clean nitrogen and then reevacuated while installing temperature probes, we reduced the measurable DC Stark effect to $-1.6(1.0) \times 10^{-18}$. Further, we found that by cleaning a large viewport we could induce another DC charge, and although we tried high power UV LEDs shining into the chamber to dissipate the charge, this method seemed much less effective than purging the system with gas. The exact mechanism for removing the charges was theorized to be water molecules contaminating the nitrogen gas. To complete the full evaluation of the DC Stark effect, we performed similar measurements along the horizontal directions and determined its effect at $-1.9(1.9) \times 10^{-18}$.

\subsubsection{Active Control}

This feed forward method of measuring the DC stark shift and then running the clock is somewhat nerve racking as compared to many of the other systematics that are either actively measured or cancelled. Recent work has shown that due to the nature of the statistics involved in equation 4.5, a lower residual field will average down faster. So by intelligently biasing the electric field plates, one can not only actively cancel the field at the atoms but also have real time information on whether this field has drifted. Recent unpublished results show that this active cancellation can indeed measure an actively cancelled shift to an uncertainty of $1 \times 10^{-19}$ in only 15 minutes of running the clock. Because of optical lattice clocks high stability, active systems like these will be the future of OLCs.

\subsection{Miscellaneous Shifts}

While we have discussed many of the major shifts of the clock transition, save the BBR shift which will be discussed in the next chapter, a great many more shifts still need to be examined. In 
any new experiment hoping to push the frontiers of frequency metrology to even higher levels of precision all of these shifts must be considered, and perhaps some that have yet to be discovered.

\subsubsection{First and Second Order Doppler Shifts}

By trapping the strontium atoms deep within the Lamb-Dicke regime, the motional state of the atoms becomes separated from the internal electronic state of the atoms. Assuming one is probing along the tightly confined direction, the Lamb-Dicke parameter can be calculated as $\eta^{2}=\frac{\omega_{\text {Recoil clock }}}{\omega_{\text {sideband }}}$, which for our case is far below 1. However, even with this condition met, first order Doppler shifts can still occur due to relative motion of the atoms with respect to the wavefront of the clock laser. For small velocities, the fractional frequency change is approximately equal to $\beta=\frac{v_{\mathrm{rel}}}{c}$, meaning even a persistent velocity of a few $\mu \mathrm{m} / \mathrm{s}$ would cause a few $10^{-15}$ shift.

Luckily, the atoms position is fixed by the lattice, and a lattice mirror can be used as a fixed reference point to the atoms. We physically connect a mirror to the same mount that holds the fixed cavity lattice mirror and use a reflection off that system as our fiber phase noise reference plane [75]. By having the wavefront phase of the clock laser referenced to the same point as the lattice via a high bandwidth transducer we make the clock laser phase follow the motion of the atoms' trap precisely. Thus canceling residual first order Doppler shifts.

Using the measured temperature of around $3 \mu \mathrm{K}$, we calculate an average total velocity of $3 \mathrm{~cm} / \mathrm{s}$. A Taylor expansion of the full relativistic Doppler shift has second-order terms from both longitudinal and transverse motion. Overall, the second-order Doppler effect results in a fractional shift less than $10^{-20}$.

\subsubsection{AOM phase chirp}

Turning on and off the clock laser light for performing detuned $\pi$ pulses can cause unexpected consequences. In the SrII experiment we decided to perform this procedure using an AOM. However, the heating, cooling, and initial formation of the phonon waves inside the AOM can cause distortion of the clean phase of the clock light [76]. To be more specific, as different parts of the AOM heat up 
in a non-uniform manner one can imagine very strange pathlength modulations, resulting in optical phase shifts. This effect was measured in SrII by the creation of a Mach-Zehnder interferometer examining the phase of the beated light on a digital phase detector [77]. To more easily measure an appreciable signal the effect of higher RF input powers into the AOM were examined. Convolving the response function of the atoms for Rabi spectroscopy (whose sensitivity peaks in the middle of the pulse) with the raw measured phase of the light shows the shift magnitude in Fig. 4.6A. Proper choice of neutral density filters allow one to operate with the intensity servo set to give the lowest shift measured $6(4) \times 10^{-19}$. I have no doubt that this effect can easily be lowered in the future by running the clock and measuring the shift at even lower RF powers.
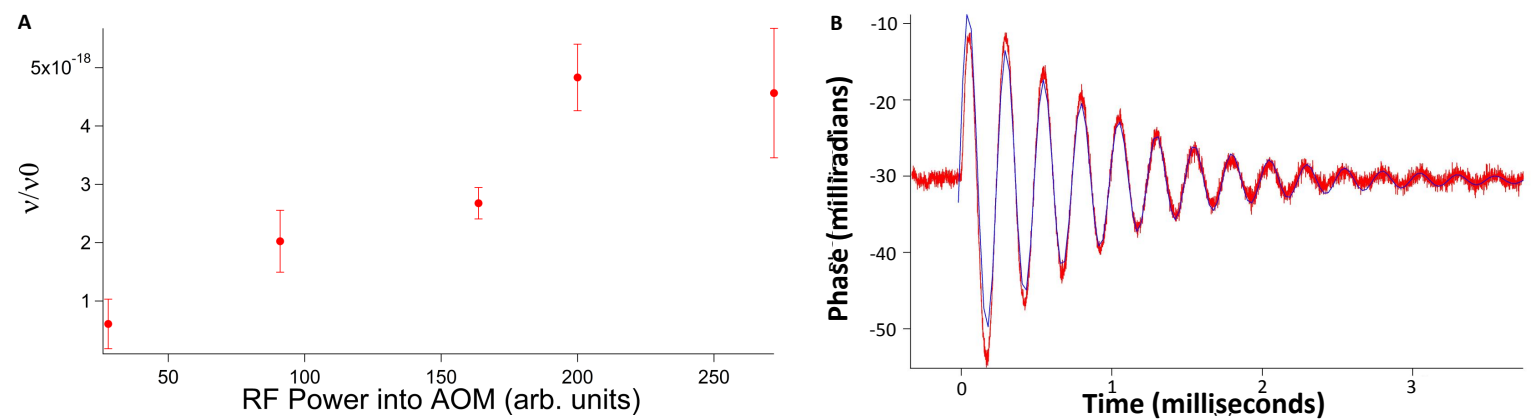

Figure 4.6: AOM Phase Chirps present in the SrII system. A) This panel shows the calculated clock shift due to the tuning AOM's phase chirp when the light is pulsed on and off. Longer interrogation times will decrease this shift even further in the future. B) The in-loop signal seen from the fiber phase noise canceller when its signal was amplitude modulated. Even with this pronounced ringing this does not produce a particularly large shift. The analysis of these effects was included in an internal Ye labs writeup written by Jason Williams.

A secondary concern was the response of the fiber phase noise canceller to pulsing the first order light off and on. This pulsing amplitude-modulates the 0th order light and subsequently the fiber phase noise signal [75]. The servo can be seen to ring at the beginning of each pulse due to AM-PM conversion in the RF mixer. Luckily, due to the insensitivity of the atoms to the beginning to the Rabi pulse, this ringing observed in figure $4.6 \mathrm{~B}$, would cause a frequency shift of only $8 \times 10^{-20}$. 


\subsubsection{Line Pulling}

Line pulling can be caused by a variety of effects. These effects include a slight ellipticity in the clock laser polarization driving detuned sigma transitions, imperfect optical pumping combined with the effect of driving a detuned $m_{F}= \pm 7 / 2$ transition, and clock laser-induced tunneling between different pancakes. Each of these effects is modeled as a deformation of the perfect Rabi lineshape. The spectral narrowness of the $160 \mathrm{~ms}(\sim 5-\mathrm{Hz})$ Fourier-limited linewidth with which all data was taken in this work greatly reduced the effect of any possible line pulling. It has been noted in recent work that line pulling effects should be modeled more formally as coherently driving a $\Lambda$ system, which similarly gives incredibly low shifts of $2 \times 10^{-19}$ for extremely conservative parameter estimates [26].

\subsubsection{Probe Beam AC Stark}

The only exception to taking data with spectrally narrow features was the investigation of the AC Stark shift induced by the clock laser itself. This systematic was evaluated by measuring the frequency difference between our clock transition interrogated with 50-ms and 200-ms $\pi$ pulses. For this measurement, the clock was run with our largest possible bias magnetic field to avoid any residual line-pulling effects during the 50-ms clock interrogation. Including the errors in determining our $\pi$ pulse exactly, we estimate a $1.3 \times 10^{-18}$ uncertainty in the probe beam AC stark shift for SrII's normal clock operation. More recent experiments to pin this systematic down even further have found a host of systematics related to taking this measurement. However, recent work with a HyperRamsey interrogation scheme with the octupole transition in $\mathrm{Yb}$ ions has shown that even strong probe beam AC stark shifts can be cancelled to incredibly high precision [78, 79]. This technique involves a detuned and time-imbalanced spin echo interrogation sequence to actively cancel the probe beam AC stark induced by driving the weak transition. Future experiments should take this last information to heart and consider revisiting bosonic strontium clocks should the line $\mathrm{Q}$ or atom number ever turn out to be a limitation. A main detraction for ${ }^{88} \mathrm{Sr}$ clocks is 
the need to greatly power broaden the incredibly weak synthetic clock state created by applying a state mixing magnetic field.

\subsubsection{Background Gas Collisions}

Two different methods were examined to quote an uncertainty for background gas collisions. The first method was to scale down the shifts measured in Ref. [80]. However, these measurements were not performed using hydrogen gas. By far the largest residual gas in our ultrahigh-vacuum, oven-loaded, vacuum system is hydrogen. The second method followed the procedure outlined in Ref. [81] and was what was included in Ref. [22]. Differential $\mathrm{C}_{6}$ coefficients for the $\mathrm{Sr}^{1} \mathrm{~S}_{0}$ and ${ }^{3} \mathrm{P}_{0}$ states for their resonant dipole interactions were scaled to the Cs ground state - ground-state $\mathrm{C}_{6}$ coefficients [82]. Both the $\mathrm{Sr}^{1} S_{0}-\mathrm{H}_{2} \mathrm{C}_{6}$ coefficient and the $\mathrm{Sr}{ }^{3} P_{0}-\mathrm{H}_{2} \mathrm{C}_{6}$ coefficient were then estimated by scaling with respect to the non-resonant dipole $\mathrm{CsH}_{2} \mathrm{C}_{6}$ coefficient. Atomic trapping lifetimes of about $1 \mathrm{~s}$, average excitations during the detuned Rabi pulse, and interrogation times of $160 \mathrm{~ms}$ were combined to estimate the background gas collisional shift uncertainty. In our final uncertainty budget, no shift correction is quoted, and we provide only an upper bound of this uncertainty. 


\section{Chapter 5}

\section{$\mathrm{Sr}$ - The mini-COBE of Clocks}

The Nobel prize in physics in 2006 was given to the Cosmic Background Explorer (COBE). One of the key features of this experiment was the measurement of the background blackbody radiation left over from the early stages of the universe. Interestingly, the last systematic to be discussed in a neutral atom clock has a lot in common with the COBE satellite. While we normally consider our atoms to be trapped inside a nearly perfect vacuum, with nothing but a few stray hydrogen molecules bouncing off the walls, the truth is all the components of our vacuum chamber are sitting at room temperature spewing out blackbody photons throughout the entire EM spectrum. Luckily, the spectrum peaks at about $10 \mu \mathrm{m}$ for room temperature, $298 \mathrm{~K}$, which is incredibly far detuned from many of the atoms' electronic transitions.

While there is very little resonant light contained in the BBR spectrum of $298 \mathrm{~K}$, it behooves us to examine how poorly all leading atomic clocks are shifted by the broadband radiation. As can be seen in table 5.1, strontium is seemingly the most difficult neutral atom to create an optical lattice clock with. Of course this is a trade off, because strontium has more transitions in the visible and NIR it is more susceptible to BBR radiation but easier to work with than say neutral mercury. Compared to the alkali atoms used for RF clocks, strontium just barely has the upper hand on BBR-related shifts. However lost in the numbers is the necessarily simpler system that a strontium OLC apparatus has compared to a fountain clock. The atoms in an OLC stay in one place during the interrogation, experiencing only one temperature environment whereas the cesium atoms in a primary standard are thrown upwards into an elongated chamber being exposed to a 
Table 5.1: BBR shift magnitudes for room temperature radiation for a variety of atomic species. Reproduced from ref. [83] unless otherwise cited.

\begin{tabular}{c|c||c}
\hline Atom & Clock Transition & $\delta \nu / \nu_{0}$ \\
\hline${ }^{87} \mathrm{Rb}$ & $5 s(F=2-F=3)$ & $-1.3 \times 10^{-14}$ \\
${ }^{133} \mathrm{Cs}$ & $6 s(F=4-F=3)$ & $-1.7 \times 10^{-14}$ \\
\hline $\mathrm{Ca}^{+}$ & ${ }^{2} S_{1 / 2}-{ }^{2} D_{5 / 2}$ & $9.2 \times 10^{-16}$ \\
$\mathrm{Sr}^{+}$ & ${ }^{2} S_{1 / 2}-{ }^{2} D_{5 / 2}$ & $5.6 \times 10^{-16}$ \\
$\mathrm{Al}^{+}$ & ${ }^{1} S_{0}-{ }^{3} P_{0}$ & $-9 \times 10^{-18}[21]$ \\
$\mathrm{Yb}^{+}$ & ${ }^{2} S_{1 / 2}-{ }^{2} F_{7 / 2}$ & $-1.1 \times 10^{-16}[37]$ \\
\hline $\mathrm{Hg}$ & ${ }^{1} S_{0}-{ }^{3} P_{0}$ & $-1.6 \times 10^{-16}$ \\
$\mathrm{Mg}$ & ${ }^{1} S_{0}-{ }^{3} P_{0}$ & $-3.9 \times 10^{-16}$ \\
$\mathrm{Ca}$ & ${ }^{1} S_{0}-{ }^{3} P_{0}$ & $-2.6 \times 10^{-15}$ \\
$\mathrm{Yb}$ & ${ }^{1} S_{0}-{ }^{3} P_{0}$ & $-2.5 \times 10^{-15}[84]$ \\
$\mathrm{Sr}$ & ${ }^{1} S_{0}-{ }^{3} P_{0}$ & $-5.3 \times 10^{-15}[22]$ \\
\hline
\end{tabular}

range of viewing angles from many surfaces.

To conclude our investigation of the strontium optical lattice clock, I will discuss our recent efforts to solve the BBR shift uncertainty and present the final uncertainty budget for SrII, ending with the results of a SrI-SrII comparison.

\subsection{Understanding the Shift}

This shift is simply the integrated spectral response of the atoms to the blackbody radiation field

$$
\delta \nu_{i}=-\frac{2 \pi}{h} \int_{0}^{\infty} u(\omega) \alpha_{i}(\omega) d \omega
$$

where $\delta \nu_{i}$ is the frequency shift of state $i, u(\omega)$ is the spectral energy density of the BBR, and $\alpha_{i}(\omega)$ is the polarizability of the state. For a perfect blackbody spectra, after much manipulation this integral can be replaced with an asymptotic expansion in terms of the transition frequency between resonant states $i$ and $l, \omega_{i l}$, and the temperature $T$ in terms of the quantity $\frac{T}{\omega_{i l}}[85,86]$. Summing over all the resonances in the atom, the final result is a decreasing series of contributions that go like $T^{4}, T^{6}, T^{8}$ and so on. Not much intuition can be gained by this however, and a closer examination of the first two terms in this series is warranted as they are the only terms that are 
statistically relevant at our targeted accuracy level.

The blackbody radiation shift, given by the $T^{4}$ and $T^{6}$ terms, is most easily understood as two distinct effects acting upon the atom. The first effect, known as the BBR static shift, encompasses the rectification of the radiation to an effective DC electric field experienced by the atoms. Over $90 \%$ of the shift is encompassed in this term alone, and this is why it was so instrumental for the PTB group to measure the DC atomic polarizability of both the ${ }^{1} \mathrm{~S}_{0}$ and the ${ }^{3} \mathrm{P}_{0}$ states [74]. The second effect, known as the BBR dynamic shift, is the frequency-weighted spectrum of the radiation and accounts for photon occupancy at and near specific transitions that occur between the two aforementioned states and various other higher excited states in the atom. Assuming a perfect BBR environment, a very convenient closed-form simplification of the BBR shift is written below from Ref. [74].

$$
\delta \nu_{\mathrm{BBR}}=\nu_{\mathrm{stat}}\left(\frac{T}{300 \mathrm{~K}}\right)^{4}+\nu_{\mathrm{dyn}}\left(\frac{T}{300 \mathrm{~K}}\right)^{6}
$$

Throughout the course of this work we will take $\nu_{\text {stat }}=-2.13023(6) \mathrm{Hz}$ and $\nu_{\text {dyn }}=0.1484(16) \mathrm{Hz}$, which for the latter is the mean between the two most recent theory derivations of this shift coefficient $^{1}[74,87]$. The shift coefficient is also lent more credence due to the recent work measuring the dynamic shift as the atoms' surrounding temperature was varied from $95 \mathrm{~K}$ to room temperature, where a fitted shift coefficient of $\nu_{\mathrm{dyn}}=0.1480(26) \mathrm{Hz}$ was obtained [88]. Disregarding the uncertainty in the coefficients themselves, blindly achieving $4 \times 10^{-18}$ uncertainty in either of these shifts would be possible with $60 \mathrm{mK}$ temperature uncertainty for the static shift and $580 \mathrm{mK}$ temperature uncertainty for the dynamic shift. While these numbers might at first seem intimidating, research will quickly show that even commercial off-the-shelf temperature sensors are capable of beating these limits, precisely because of the compact nature of the Sr vacuum chamber.

Before we embark on how we use equation 5.2 to get to our low BBR uncertainty, it's important to better understand the relationship between these coefficients and their underlying physics. Examining the static shift, it should be apparent from the $T^{4}$ scaling of the shift that there is a direct scaling relationship between the static shift and the Stefan-Boltzmann law for

\footnotetext{
${ }^{1}$ The error is the difference between these two most recent values.
} 
measuring power emitted from a blackbody. For consistency, I will use the notation from Ref. [89].

$$
\nu_{\text {stat }}=-\frac{\Delta \alpha}{2 h}\left\langle E^{2}\right\rangle=(\text { For a Perfect BB Spectrum }) \frac{\Delta \alpha 4 \pi^{5} k_{B}^{4}}{15 c_{0}^{3} \epsilon_{0} h^{4}} T^{4}
$$

Realizing the connection between these two ideas, that (1) the cause of the static shift is proportional to $\left\langle E^{2}\right\rangle$ and that (2) this quantity is proportional to the heat exchanged between radiatively coupled objects, was a huge leap forward in our understanding of the BBR shift. Fundamentally, this allowed us to make the cognitive leap that a successful measurement of an object in equilibrium with its surroundings would shed light on the actual shift experienced by the atoms. But how does this relate to the frequency-weighted spectrum, namely the dynamic shift?

While the vast majority of this dynamic shift comes solely from the state with the lowest transition frequency, ${ }^{3} \mathrm{P}_{0}-{ }^{3} D_{1}$ at 2.6 microns, I will nonetheless write the more general expression that takes into account all of the couplings [89].

$$
\nu_{i, \mathrm{dyn}}=-\frac{1}{(2 \pi)^{2}}\left(\frac{k_{B} T}{\hbar}\right)^{3} \sum_{k} \frac{2 J_{k}+1}{2 J_{i}+1} \frac{A_{k i}}{\omega_{i k}^{3}} G\left(\frac{\hbar \omega_{i k}}{k_{B} T}\right)
$$

where $G(y)=\int_{0}^{\infty} \frac{x^{3}}{e^{x}-1}\left(\frac{1}{y-x}+\frac{1}{y+x}-\frac{2}{y}\right) d x, A_{k i}$ refers to specific transition's Einstein A coeffecients, and $\nu_{\mathrm{dyn}}=\nu_{\mathrm{e}, \mathrm{dyn}}-\nu_{\mathrm{g} \text {, dyn }}$. This equation can of course be transformed back into an equation based solely on the electric field of the radiation, allowing easy use of frequency dependent emissivities and custom spectra. However, many people like the use of the function $G$ as it is very similar to the function $F_{1}$ introduced by Farley and Wing, which allows for an asymptotic expansion of these integrals for a perfect BBR spectrum $[90,89,87,86]$. As an aside for future BBR modelers, while these substitutions might be convenient, Mathematica, with a little bit of hand holding, can perform these integrals regardless of the singularities present, and Matlab's quadgk function works on the first try. Understanding that an arbitrary electric field's effect on $\delta \nu_{\mathrm{BBR}}$ can be calculated, it remains for us to understand exactly what kind of spectra the atoms see, how both the static shift and the dynamic shift diverge from the perfect BBR shift, and most importantly how their divergences are correlated.

Taking inspiration from an older paper, not about BBR shifts, but about metastable state lifetimes, we found references to simple techniques for evaluating what sample BBR spectra could 
look like [91, 92]. The methods presented and used in these two papers can be distilled into an exercise in photon counting. By tabulating all the surrounding surfaces where photons can come from and keeping track of those surfaces' temperatures and emissivities one can write down their emissivity weighted solid angles and an approximation of the BBR spectrum experienced at any point inside a closed object. However, absolute knowledge of an effective temperature requires extremely detailed knowledge of emissivities of surfaces at a multitude of angles and frequencies. Playing around with these emissivity weighted solid angles one should quickly see that without this knowledge it would be fruitless to try and claim knowledge of an absolute effective temperature known from even these simple models.

While previous experiments have sufficed with placing temperature sensors surrounding their chambers, additional knowledge seems to be required for achieving lower BBR uncertainties. In fact, while it has been recently argued that these temperature sensors create a boxcar like uncertainty allowing for the use of a special rule in the BIPM's 'GUM: Guide to the Expression of Uncertainty in Measurement' which allows for a rather low quoted uncertainty, I do not agree with its use in this case $[26,93]$. Furthermore, the GUM's use of this rule comes with two caveats found in Section 4.3.7 [93]. First, one must have a uniform probability of finding the value in the boxcar distribution, which I argue is incorrect as components with high emissivity will dominate the temperature felt by the atoms. Second, it is noted that this method should not be used if the results of this uncertainty are considered particularly important to the overall result, which for the case of the leading systematic in an OLC it is. Measurements only on the outside of the chamber make it incredibly difficult, even with aggressive temperature modulations and state of the art lasers to estimate the spectrum seen by the atoms. Instead, we would need to combine our understanding of the spectrum with a knowledge about what is going on internally in the vacuum chamber.

\subsubsection{Ray Tracing Model}

Creating an emissivity weighted solid angle distribution from the very center of the chamber is nothing more than a complicated geometry problem. Our extended octagon from Kimball Physics 
(Part No. MCF800-ExtOct-G2C8A16) has very detailed dimensions. Sitting down with a notebook and calculating what each and every components solid angle is from the very center of the chamber is easily accomplished. But what about slightly off-center? Or what if one would like to see the modeled spectrum for a variety of positions around the chamber? Indeed, as we will see, knowledge of temperature gradients throughout the system will shine light on the non-perfectness of blackbody radiation spectrum.

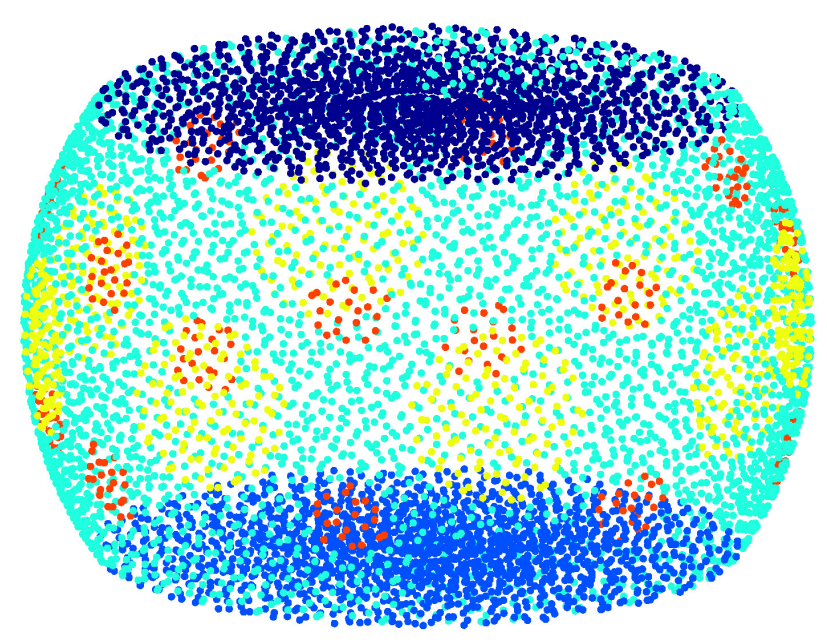

Figure 5.1: Ray tracing model of the UHV vacuum chamber. The point of origin for these rays is the exact center of the chamber. Each colored dot represents a ray hitting a surface of the chamber. Different surfaces have been color coded.

To model the chamber, I decided to use a custom ray tracing program. While many options exist for writing a ray tracing algorithm, I decided to write what I thought was the most conceptually simple ray tracing algorithm. Rays would be cast out of a point in (x,y,z) space inside of the chamber at a specified direction. The chamber's boundaries would be specified inside an auxiliary function, and binary search would be performed on the point of origin and some maximum distance away along the ray to find the intersection between the chamber boundaries and the light ray. The intersection is then noted and the surface that is struck by the ray is written down. Although this procedure turned out to be very inefficient, reasonable results could be obtained on a few year old laptop in about 20 minutes per origin point ${ }^{2}$. Even though one's first instinct might be to cast the

\footnotetext{
${ }^{2}$ Hindsight being $20 / 20$, a quick web search on how to perform ray tracing analysis will reveal a myriad number
} 
Table 5.2: Effective solid angles when sitting at the exact center of the chamber. Although these number do not include first-order reflections, these reflections will just reemphasize the importance of high emissivity temperature components like the top and bottom viewports.

\begin{tabular}{c|c||c}
\hline Name of component & Number of components visible & Total Effective Solid Angle \\
\hline Top Viewport & 1 & $37.4 \%$ \\
Bottom Viewport & 1 & $37.4 \%$ \\
Electropolished Metal Chamber & 1 & $0.3 \%$ \\
$2^{3 / 4 " \text { Viewport }}$ & 8 & $17.9 \%$ \\
1 1/3" Viewport & 15 & $6.7 \%$ \\
Zeeman Slower Window & 1 & $0.5 \%$ \\
\hline
\end{tabular}

rays using a Monte Carlo algorithm, studying the error associated with estimating solid angles via this method quickly showed that this method required significantly more rays. Instead, an iterative method known as the Hammersley point sets were used [94]. The results can be seen in figure 5.1 where a color coded representation of the main vacuum chamber shows where all the rays from the exact center have struck.

Using emissivities from Ref. [91] and the viewport manufacturers specifications combined with temperatures measured on the outside of our chamber we can start to get an idea about the kinds of spectra inside. Looking at table 5.2 recounting the emissivity-weighted solid angles as seen from the very center of the chamber a few things become clear. First, because of the high emissivity of top and bottom viewports $(\sim 0.92)$ and how close those two viewports are to the atoms they play a huge role in determining the radiation spectra seen by the atoms. Second, the low effective solid angle of the metal chamber due to its lower modeled emissivity $(\sim 0.003)$ means the temperature will be dominated solely by the temperature of the various viewports on the system. One might worry that because these emissivity values are soft that we might not be gaining any useful information from this exercise.

To see why we can gain an upper hand on this problem, let us instead focus on what an ideal radiatively coupled temperature probe might read out. This ideal temperature probe would of more efficient algorithms for encoding and traversing environments to perform ray tracing. 
exchange radiative power with its environment, and its response to any arbitrary spectra can be calculated. In fact, as the temperature probe reads out a specific number, we have fundamentally gained some understanding of the intravacuum spectra. Although, as an experimentalist given only this one number, it would be impossible to figure out how poorly the spectra matches a perfect Planck spectrum. However, by varying parameters in the model and keeping track of both the assumed perfect BBR temperature read out by the ideal sensor and the actual shift caused by the modelled spectra we can examine how useful a perfect BBR sensor would be. Surprisingly, because of small temperature differentials $(3 \mathrm{~K})$ across our chamber, the emissivities of the metal or the viewports would need to change by a factor of 20 to introduce a $1 \times 10^{-18}$ error into the temperature sensor's shift errors. This means that even with very little knowledge of component emissivities, an ideal temperature probe, under the right conditions, would measure the temperature accurately enough for our purposes. Furthermore, by taking temperature measurements outside of the system on viewports and moving the intravacuum sensor around to measure temperature gradients the model can be validated ${ }^{3}$.

\subsection{The Temperature Sensors}

The gauntlet had been thrown, keep temperature differentials small and try to create an ideal and accurate set of temperature sensors in situ. In a wholly uncharacteristic show of cooperation from the experimental apparatus, we were lucky to find that our carefully built stray-light enclosure acted as a strong temperature homogeneity enforcer for the entire system. Fueled mostly by warm exhaust coming out of a CCD camera cooling unit, after closing the enclosure in the morning, temperature probes all around the system and hanging in the enclosure slowly came to equilibrium at about $301 \mathrm{~K}$ with at the very most $3 \mathrm{~K}$ temperature differential between the minimum and the maximum registered temperatures. As a further benefit, long comparison measurements over days clearly showed the lab temperature swinging by many degrees, effecting the SrI apparatus, while SrII only registered a drift of $\sim 200 \mathrm{mK}$ in its enclosure.

\footnotetext{
${ }^{3}$ Similarly, one could just have many spatially separated temperature probes.
} 
Many obstacles exist in taking a good measurement of temperature, and I would be remiss to not give special thanks to Hans Green for designing and building the mounts for our temperature sensors, as it was those mounts that allowed us to accurately measure the temperature as well as we did. While we ended up using temperature sensors that had previously been purchased for another project, I will nonetheless make the case for their use. Future theses will go significantly more in depth on this issue. Many different kinds of sensors exist to measure temperature: thermistors, platinum resistance temperature detectors (RTDs), crystal oscillators, and silicon diodes. While it might be that platinum RTDs are the more conservative choice, silicon diodes offer a few advantages which seem to minimize their systematics. First, silicon diode sensors have a very linear response in voltage vs temperature making their calibration easier [95]. Second, the signal size of $\sim 0.5 \mathrm{~V}$ for a very tiny current make their readout much easier, and more immune to contact resistances as the effective resistance of the diode is quite high. Third, compared to RTDs which can dissipate $100 \mu$ Watts during operation with self-heating on the order of $\sim 0.4 \mathrm{mK} / \mu \mathrm{W}$, diodes dissipate 5 $\mu$ Watts with self-heating coefficients close to $\sim 0.2 \mathrm{mK} / \mu \mathrm{W}$ making their calibrations more immune to differences in mounting ${ }^{4}$. With these considerations in mind we used a DT-471 calibrated sensor from Lakeshore Cryotronics.

To decrease heat conduction from the chamber to the temperature sensor, two diodes were mounted at the end of two elongated glass tubes. These glass proboscises also allow the tube itself to radiatively couple to the environment. Figure $1.2 \mathrm{c}$ shows a CAD drawing of the two sensor mounts, both a retractable movable mount and a shorter fixed mount, while figure 5.2 shows a photograph of a sensor. The metal conflat flange to glass tip distance on the movable sensor is $150.6 \mathrm{~mm}$ while the fixed sensor is $85.2 \mathrm{~mm}^{5}$. The pyrex glass that the mount is made of has a $7 \mathrm{~mm}$ diameter with $1 \mathrm{~mm}$ thick walls. The diode sensor is epoxied onto a short glass platform at the end of the tube using Epotek 353ND. The leads of the sensor are then silver soldered with

\footnotetext{
${ }^{4}$ The SD package for our diodes also places the diode junction inside a relatively gigantic thermal load further reducing systematic errors in the calibration.

${ }^{5}$ While this was the design goal, full extension of the movable sensor seemed to place the sensor slightly past the center of the chamber by $\sim 8 \mathrm{~mm}$.
} 


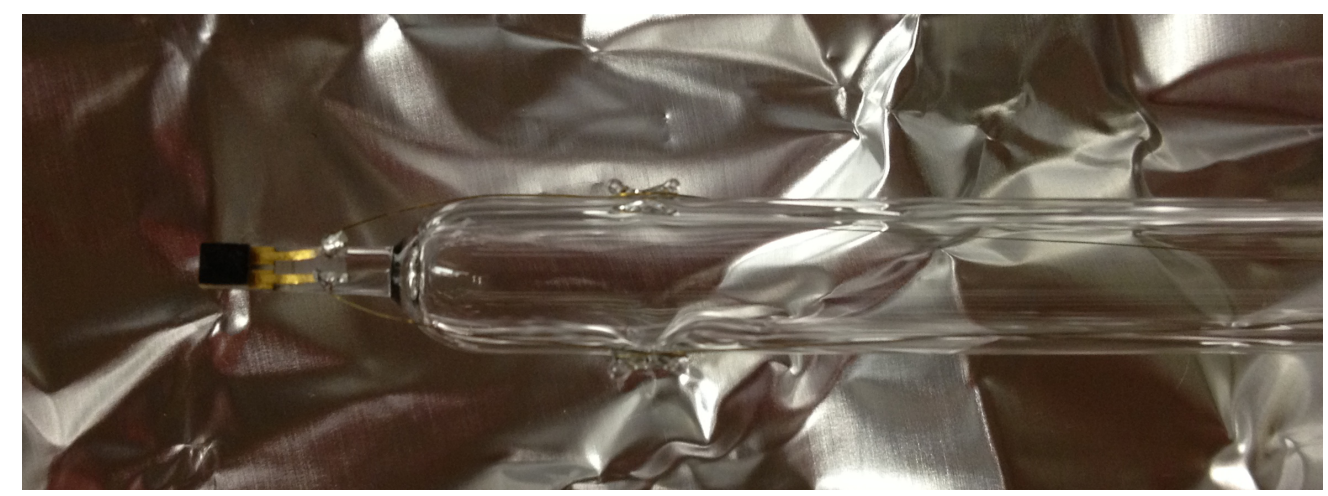

Figure 5.2: A picture of the diode temperature sensor mounted on the pyrex glass proboscis. The top facet of the diode has been painted with high emissivity vacuum compatible paint. One can see the silver soldered phosphor bronze wires leading away from the diode strain relieved on special glass tie downs. A CAD model of the two sensors is depicted in figure 1.2C. 
Table 5.3: Diode temperature sensor corrections and uncertainties.

\begin{tabular}{c|c||c} 
Corrections & $\Delta \mathrm{T}(\mathrm{mK})$ & $\sigma_{\mathrm{T}}(\mathrm{mK})$ \\
\hline Calibration (including self-heating) & 0 & 16 \\
Residual conduction & 0 & 0.7 \\
Temperature gradient & 40 & 20 \\
Lead resistance & 7.7 & 1.5 \\
Lattice light heating & -15 & 7.5 \\
\hline Totals & 32.7 & 26.7 \\
\hline
\end{tabular}

Fusion solder paste WCC-430-801 made up of 96.5\% Sn and 3.5\% Ag to phosphor bronze leads. The lead material is thermally insulating and fairly electrically conductive. To increase radiative coupling, facets of the sensor that could be painted with high emissivity vacuum-compatible paint were coated with Ball VacKote 48816. Because of the high-emissivity of the glass, this is less of a requirement, however it very likely increases the sensor's radiative coupling. The phosphor bronze leads are strain relieved around notches in the glass, and wind around the proboscises towards the metal flange where they are connected to vacuum compatible electrical feedthroughs.

Vacuum baking of the sensor was performed before installation of the sensor into the main UHV trapping chamber. Unfortunately, the baking temperature was limited to $200{ }^{\circ} \mathrm{C}$ by the silver solder, not the sensor itself. Although care was taken to not stress the sensor leads, the movable sensor was sadly struck off its holder causing a severe change in its calibration. Later tests comparing the sensors showed that relative temperature measurements below a few tens of degrees were still possible with the broken sensor, but absolute measurements would require a complete sensor recalibration. Upon installation into the chamber re-baking of the sensor to $100{ }^{\circ} \mathrm{C}$ was required to pump down to $5 \times 10^{-10}$ Torr at the system's ion gauge. While originally theorized to be an issue with the porousness of the high-emissivity paint, newer work has suggested that the sensor packaging itself, or at least the damaged sensor, might create a virtual leak by trapping a small amount of water vapor or gas.

Many sources of error for extrapolating the temperature at the atoms needed to be addressed 
to take the measurement from the fixed sensor and extrapolate the temperature at the atoms. Reading table 5.3 one will understand some of the largest systematics we investigated. Sensor calibration which was done by LakeShore, claimed an accuracy of $16 \mathrm{mK}$ at $300 \mathrm{~K}$. Although this seems small, this is actually less than a $1 \mathrm{ppm}$ measurement accuracy, state-of-the-art temperature measurements near the triple-point of water can reach as low an uncertainty as a few $\mu \mathrm{K}$ [96]. Residual conduction was measured by wrapping the conflat flange of the fixed sensor in nichrome wire and heating up the flange by ten degrees. Although previous experiments showed rapid response of the sensor to changes in high-emissivity objects' temperature, no clear signal due to heat conduction was seen after taking data for many minutes. An uncertainty was made by taking the peak to peak amplitude measured throughout the experiment and scaling that number down linearly with normal temperature differentials between the flange and the sensor. A temperature gradient from the retracted movable sensor's position to its extended position where the atoms would normally sit was measured with the system running, but the oven blocked. Auxilary temperature sensors confirmed that the bottom viewport was slightly warmer than the top viewport, most likely due to insufficient airflow inside the enclosure. Although the model and the measurement agreed on the magnitude of the gradient, a conservative uncertainty of $50 \%$ was added to this systematic. Lead resistance and its effect on the measured voltage was estimated from the length of the phosphor bronze wires. Finally, stray lattice light scatter caused a very clear $15 \mathrm{mK}$ change in the measured sensor's temperature, again a conservative uncertainty of $50 \%$ was added. Other systematics like magnetic field effects or thermocouple effects in the vacuum feedthroughs were tested or researched, but were found not to be an issue.

A few simple tests of the emissivity-weighted solid angle model revealed surprisingly good results. In fact, measurements around the chamber and of the top and bottom viewport predicted an absolute temperature within $100 \mathrm{mK}$ of the measured temperature. However, these results were too dependent on the emissivities to make this a viable temperature measurement technique without careful study of the material properties. Agreement was also found when the movable sensor was moved into view of the Zeeman slower window with the heater still on (see Fig. 5.3). Not only 
did the broad shape of this Zeeman slower window gradient match what the model predicted, the peak height of $30 \mathrm{mK}$ temperature increase also matched predictions. Keeping the Zeeman slower window at $150{ }^{\circ} \mathrm{C}$ however, would create a $>1 \times 10^{-18}$ spectrum shift due to the very different temperature of the window with respect to the rest of the chamber. Lowering the temperature to $12^{\circ} 5 \mathrm{C}$ would make the spectrum shift lower than $1 \times 10^{-18}$, however this would place a fairly large emphasis on modeling and its associated uncertainties. Instead, one can just run the clock for short periods of time without the window heated, or obscure the atoms' view of this radiation.

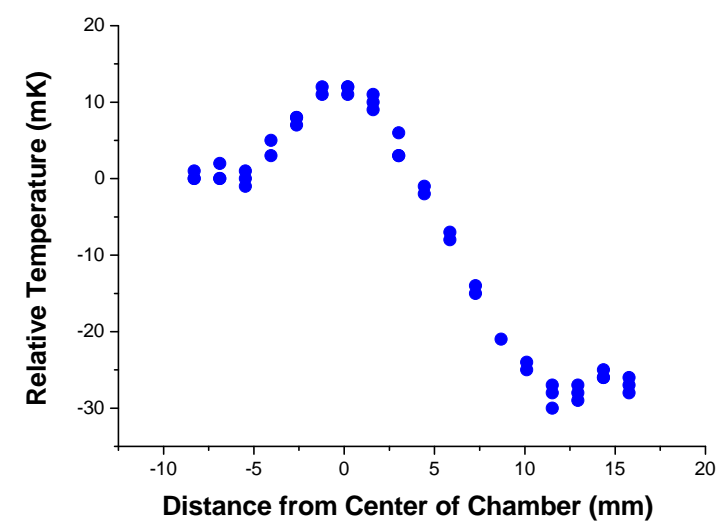

Figure 5.3: A measurement of the influence of the Zeeman slower heated window on the temperature sensor. Although this measurement was not taken under running conditions for the clock, a broad spatial gradient can be seen of at least $30 \mathrm{mK}$, in addition to the peaked response of the sensor when placed in view of the Zeeman slower window.

Putting all this information together we get an approximately $\sim 27 \mathrm{mK}$ temperature uncertainty in our measurement. While this is actually better than we initially hoped, the uncertainty in the BBR dynamic coefficient far exceeds the residual uncertainty in the temperature measurement. To decrease the coefficient uncertainty future experiments will need to measure the Einstein A coefficient for the ${ }^{3} \mathrm{P}_{0}-{ }^{3} \mathrm{D}_{1}$ transition at 2.6 microns. While work can be done to further decrease the uncertainty, via better sensor calibration techniques, this method will seem to bottom out after only a factor of a few improvement. Future clock work to reach uncertainties in the $10^{-19}$ will most likely be accomplished by trapping the atoms in cryogenic environments specifically designed to 
occlude temperature inhomogeneities or through the use of other atomic species.

\subsection{Final SrII Error Budget}

Tabulating all the systematic uncertainties discussed in the previous two chapters we can combine them to get a rather astonishing result. As seen in table 5.4 the total systematic uncertainty for SrII is $6.4 \times 10^{-18}$, this represented the first time an OLC held the mantle of the lowest total systematic uncertainty. While this result is a welcome sight for the OLC community, it's interesting to reexamine the measurement with some hindsight. The two leading systematics that make up the vast majority of this number come from the dynamic BBR coefficient and the AC stark effect. Both of these are solvable problems that do not interfere with this method of temperature readout. As such, a renewed measurement of the AC stark effect with a larger lever arm (more powerful pump laser) and a lower trap operating depth (vertical lattice) will allow SrII to squash that systematic down. Secondly, a lifetime measurement of the ${ }^{3} D_{1}$ line will help pin down the dynamic BBR coefficient. In the end, following these two simple prescriptions should cut the total SrII frequency uncertainty down by a factor of 2 at least. Work along this line is ongoing in the lab.

\subsubsection{SrI-SrII comparison}

As the systematic evaluation of SrII was coming to a close, SrI, led by Mike Bishof and Xibo Zhang, took up the challenge of a systematic evaluation allowing us to perform clock-clock comparisons. Since this research is meant to highlight the utility of high stability clocks, it was particularly important for us to perform a clock comparison between two OLCs. In fact, the low instability of these Sr clocks $\left(3 \times 10^{-18}\right.$ at about 10,000 s), displayed as the Allan deviation of their frequency comparison in Fig. 5.4a, is perhaps their greatest selling point.

Figure 5.4b documents a comparison of the SrI and SrII clocks over a period of one month. After the data from the comparisons had been post-processed by each individual strontium team, time-stamped and corrected frequencies were shared. Although the overall systematic uncertainty

of the comparison is $5.4 \times 10^{-17}$, as a consistency check for the comparison a variety of methods 
Table 5.4: SrII Systematic Uncertainties. Shifts and uncertainties are given in fractional frequency units multiplied by $10^{-18}$. Uncertainties are quoted as $1 \sigma$ standard errors. They are determined with the square root of the quadrature sum of the systematic error and statistical error, with the latter quantity inflated by $\sqrt{\chi_{\nu}^{2}}$. Reproduced from ref. [22].

\begin{tabular}{c|c||c}
\hline Shifts & $\Delta \nu$ & $\sigma_{\nu}$ \\
\hline BBR Static & -4962.9 & 1.8 \\
BBR Dynamic & -345.7 & 3.7 \\
Density Shift & -4.7 & 0.6 \\
Lattice Stark & -461.5 & 3.7 \\
Probe Beam AC stark & 0.8 & 1.3 \\
First-order Zeeman & -0.2 & 1.1 \\
Second-order Zeeman & -144.5 & 1.2 \\
Residual Lattice Vector & 0 & $<0.1$ \\
Line Pulling and Tunneling & 0 & $<0.1$ \\
DC Stark & -3.5 & 2.1 \\
Background Gas Collisions & 0 & 0.6 \\
AOM Phase Chirp & 0.6 & 0.4 \\
Second-order Doppler & 0 & $<0.1$ \\
Servo Error & 0.4 & 0.6 \\
\hline Totals & -5921.2 & 6.4 \\
\hline
\end{tabular}



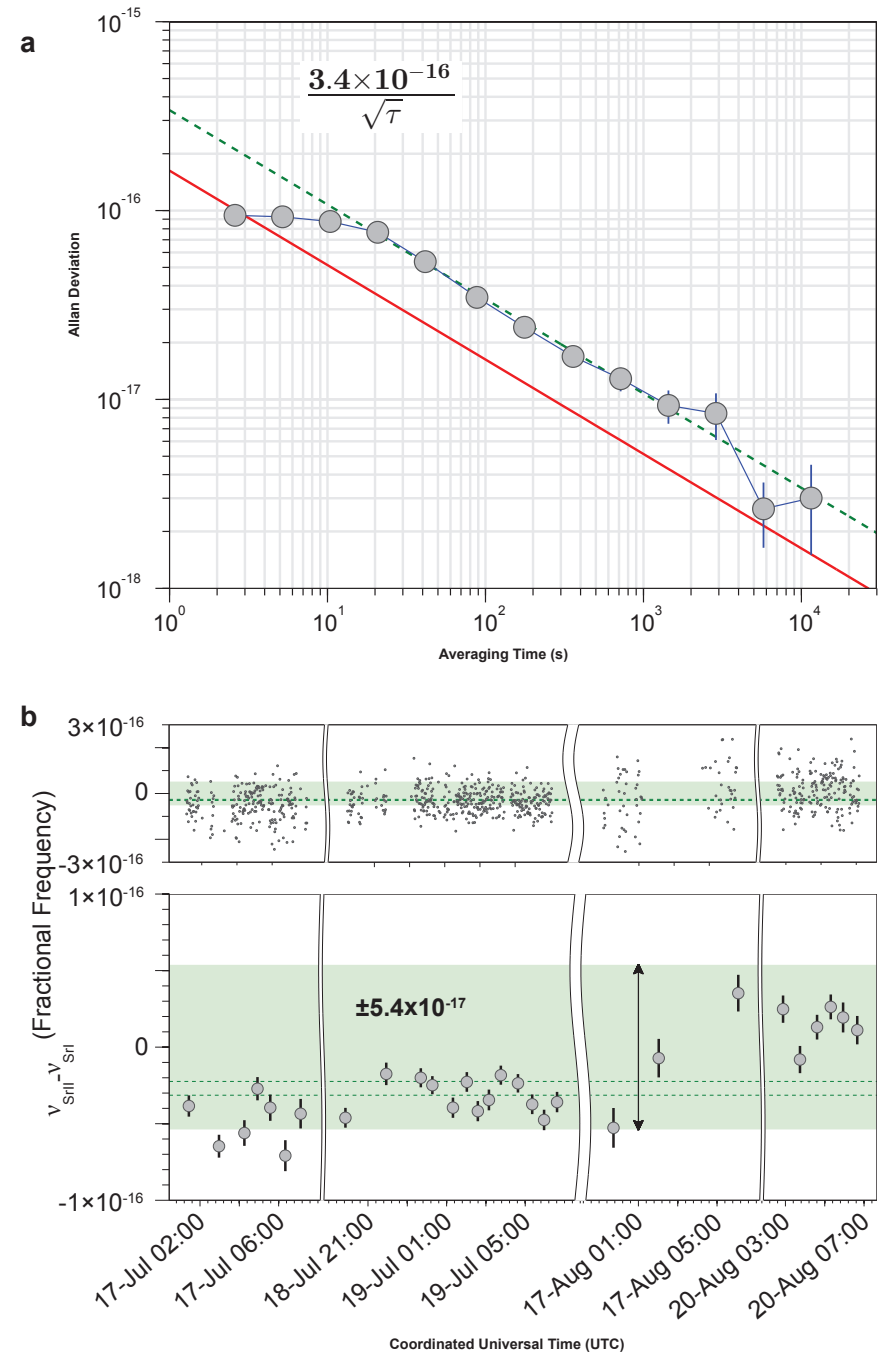

Figure 5.4: SrII - SrI Frequency Comparison. a) Allan deviation of the SrI and SrII comparison divided by $\sqrt{(} 2)$ to reflect the performance of a single clock. The red solid line is the calculated quantum projection noise for this comparison. The green dashed line is a fit to the data, showing the worst case scenario for the averaging of a single clock of $3.4 \times 10^{-16}$ at one second. b) The absolute agreement between SrI and SrII recorded at the indicated Coordinated Universal Time. The light-green region denotes the $1 \sigma$ combined systematic uncertainty for the two clocks under the running conditions at that time. The top panel shows the frequency record binned at $60 \mathrm{~s}$; in the bottom panel each solid circle represents $30 \mathrm{~min}$ of averaged data. The green dashed lines represent the $1 \sigma$ standard error inflated by the square root of the reduced chi-squared, $\sqrt{\chi_{\nu}^{2}}$, for the weighted mean of these binned comparison data. The final comparison over 52,000 s of data showed agreement at $-2.7(5) \times 10^{-17}\left(\sqrt{\chi_{\nu}^{2}}=10.5\right)$ for the 30-min averaging time and $-2.8(2) \times 10^{-17}($ $\left.\sqrt{\chi_{\nu}^{2}}=3.5\right)$ for the 60 -s averaging time. Reproduced from ref. [22]. 
were used to show the agreement of the two clocks within this confidence interval. A simple mean of all the data gives the difference between the two clocks to be $-2.4 \times 10^{-17}$. Binning the data in small chunks, of approximately one minute per data point (as in the top panel of Fig. 5.4b) gives agreement of $-2.8(2) \times 10^{-17}$. The uncertainty on this number has been inflated by $\sqrt{\chi_{\nu}^{2}}$ , as $\sqrt{\chi_{\nu}^{2}}=3.5$ denoted overscatter in the data. Binning the data in 30-min chunks (as in the bottom panel of Fig. 5.4b) clearly shows that there are systematic fluctuations still present in the comparison, with $\sqrt{\chi_{\nu}^{2}}=10.5$ and an agreement of $-2.7(5) \times 10^{-17}$. Again, this uncertainty is inflated by $\sqrt{\chi_{\nu}^{2}}$. The greater overscatter in the data at longer timescales is probably caused by imprecise knowledge of the BBR environment for SrI, which allows for fluctuations within the $1 \sigma$ comparison uncertainty.

With the successful completion of the SrII systematic evaluation and frequency comparison, it was time to look forward to new experiments with strontium. 


\section{Chapter 6}

\section{The New Sr Quantum Gas Extravaganza Machine}

The study of quantum gases is a topic riddled with misnomers. While people in the field continually refer to various systems as having a high-phase-space density, it's easy to forget that deeply degenerate samples of alkali's or alkaline earth atoms are actually at an exceedingly low density. In fact, the nitrogen molecules in air are at significantly higher spatial densities [97]. It is only in comparison with atoms' de Broglie wavelength at ultracold temperatures that the densities seem "high". It is the exquisite control and isolation of the particles in an ultracold gas that make this field so special, and allow interactions to be studied in attainable timescales. But of course, there are many experiments all around the world focusing on quantum gases of alkali atoms, so what does an alkaline earth atom have to offer to this already rich field?

In the hunt for studying and understanding strongly correlated quantum systems, alkaline earth atoms have shown a surprising path towards achieving these delicate, correlated, many-body states. Due to the separation of energy scales between quantized motional levels and interaction energies, decoupling between the internal spins and external degrees of freedom of the atoms can easily be obtained. Although this restricts our atoms to probe weak interactions, the evolution of the system can be dominated by these weak scale interactions as other single-particle dynamics can be frozen out even at thermal $(\mu \mathrm{K})$ temperatures. Given a sufficiently long probe time we can thus achieve strongly correlated dynamics. Compared to research with alkali atoms, fundamentally quantum many-body processes $[44,34]$ are accessible in this regime that would otherwise be hidden

behind entropy scales that have yet to be experimentally obtained. Further studies to better control 
and engineer these many-body states in the presence of large thermal energies might lead to more robust quantum simulation protocols and push the frontiers of quantum science towards a seemingly more classical regime.

While alkali atoms excel in their sensitivity to magnetic fields, alkaline earth atoms excel at their sensitivity to optical fields. It is true that there are trade offs in pursuing the study of an atom lacking magnetic feshbach resonances or a convenient RF transition, however strontium offers narrow linewidths for spin manipulation [98], a host of nuclear spin states [44], a closed narrow optical transition with no scatter [99], IR transitions for low recoil cooling, and the possibility of anisotropic inter-atomic interaction feshbach resonances between metastable and ground states [100]. Given the opportunities to bring forth a new tool for the study of exotic quantum phenomena, multiple groups have achieved degenerate quantum gases of strontium [101, 102]. It is my hope, that due to our group's extensive background and expertise with spectroscopy that we might offer an interesting perspective on ultracold quantum gas experiments with strontium that utilize the unique energy scales accessible in these systems.

While the task of creating a quantum gas has become somewhat routine, the level structure in strontium offers a few different paths to degeneracy. Because of strontium's rich level structure, laser cooling to quantum degeneracy is indeed an option [101]. However, the most deeply degenerate samples of strontium have continued to be created via the use of evaporation [102]. While future experiments might focus on improving these laser cooling techniques, having an apparatus that can produce quantum gases using more tried and true methods will only make things easier. Therefore, significant emphasis on vacuum lifetime was placed when designing the new SrIII apparatus. Furthermore, future experiments that seemed promising required the use of optical access at approximately 30 degree intervals $(30,60,90,120)$, putting stringent restrictions on what our main chamber would need to look like. This following chapter will shed light on the design decisions made for the SrIII apparatus, will document its construction, and will offer a glimpse at some of the many experiments possible with this new apparatus. 


\subsection{Long Vacuum Lifetime}

To ensure that quantum degeneracy could be reached with the SrIII apparatus a vacuum lifetime in the 10s of seconds would be needed. The design goals of SrII had also been for a similar vacuum lifetime, but as is usually the case first tries are for ironing out the bugs in the system. The oven region in SrII was designed with capillary tubes to collimate the hot strontium vapor, while these tubes have performed admirably (having never run out of strontium) their large surface area at an extremely high temperature presents a huge gas load for the pumps in that region [103]. And while the oven was placed farther away from the main chamber, for differential pumping concerns, atom flux at the main chamber suffered. The second bug we battled with was the low conductance path to the main chamber ion pump, this reduced the pumps ability to take care of the extra gas load from the oven/Zeeman slower region. Adding insult to injury, even though there was low conductance to the pump, the pump magnet was much too close to the atoms and MOT coils and caused a drifting magnetic field. Finally, without the inclusion of a titanium sublimation pump, and with a mostly steel chamber, there was simply not enough pumping of Hydrogen. This resulted in vacuum lifetimes of $\sim 1 \mathrm{~s}$ for base pressures of high $10^{-10}$ Torr. So with these lessons in mind, significant thought was placed into the ultimate level of vacuum that could conceivably be reached by SrIII.

The first decision to be made for SrIII was whether or not to construct a double chambered system $^{1}$. Multi-chamber systems are very popular in vapor-gas loaded systems, because they allow one to create a very small clean science chamber distinct from a dirtier atom loading area. While many schemes for atom transfer between chambers exist like pusher beams, magnetic guiding, and optical trapping, only the last scheme would work for strontium because of the lack of appreciable magnetic moment in the ground state. Optical dipole trapping would require that the first chamber have good enough vacuum to allow for $\mu \mathrm{K}$ temperatures requiring a setup as complicated as SrII was already, in addition to a new science chamber reached via translating a lens on an air-bearing

\footnotetext{
${ }^{1}$ Not counting our oven region, which is necessary due to the low vapor pressure of strontium metal, and the lack of demonstration of a chemical dispenser.
} 
stage. This level of complexity seemed not only slow, given our concern about duty cycles in clocks, but also unnecessarily complicated. Instead, we decided to focus on single-chambered UHV systems, where collection of atoms and final interrogation occurred at the same place.

A survey of single chamber designs, loaded by a Zeeman slower, came up with all steel chambers and two-point-contacted glass chambers [104]. While glass chambers were a good option, the fragility of the glass and the difficulty and expense of a nicely AR coated glass chamber were large negatives. Focusing on steel chambers, we first needed to decide how to feed atoms into our system. While our current system was performing adequately enough, we nonetheless wanted to make improvements rather than keep the status quo. Our old Zeeman slower had never really had a true bake, as the epoxy used when wrapping it had a fairly low melting point, and many groups had come up with interesting new permanent magnet slower designs that get around this problem $[105,106]$. However, during the course of researching options for our new system, a company offered to build an oven, Zeeman slower, and 2D MOT system for $\mathrm{us}^{2}$.

\section{AOSense Atomic Beam Chambers}

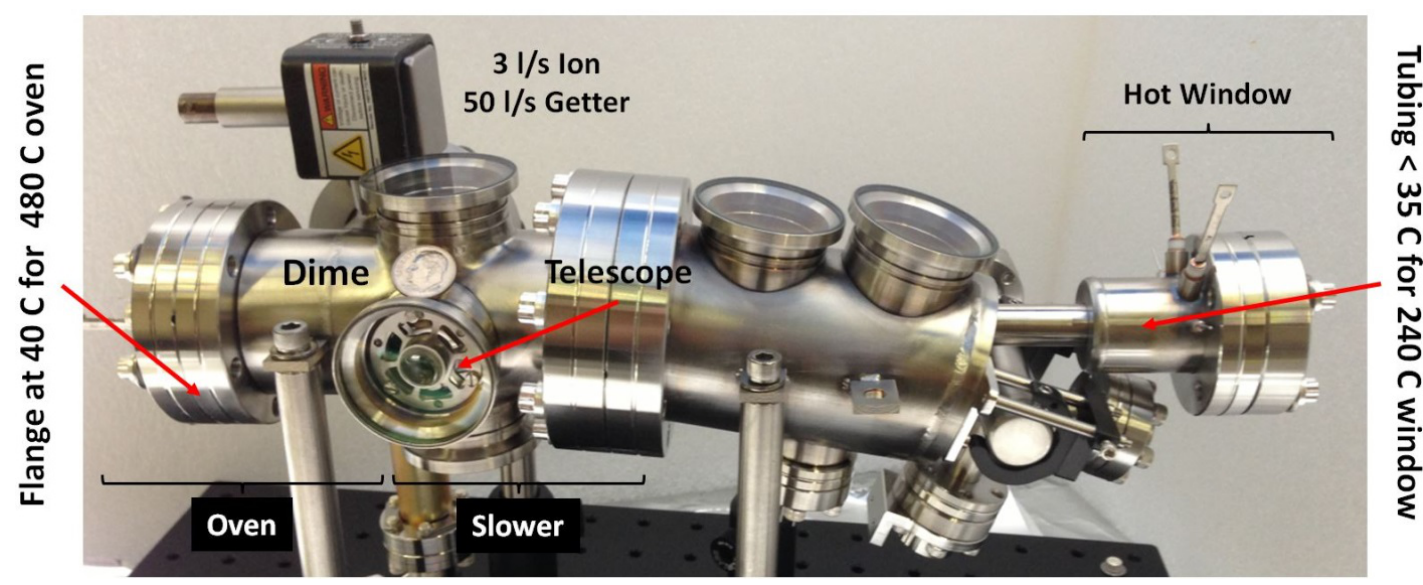

Figure 6.1: Picture of the AOSense oven, Zeeman slower, transverse cooling, and 2D-MOT system. Many of the components are intravacuum pieces like the racetrack transverse cooling system. Picture is taken from promotional material given to us by AOSense.

As can be seen in figure 6.1 the entire system has an incredibly small footprint. While detailed

\footnotetext{
${ }^{2}$ AOSense Inc.
} 
specifications can be obtained from AOSense, I would like to highlight some of the reasons why this system was attractive. First, the tiny footprint and in-vacuum oven are almost an order of magnitude smaller than the SrII oven region, leaving lots of table space for other optics and lasers. Second, the oven successfully uses capillary tubes made out of a non-stainless-steel material that outgasses significantly less. Third, not only is there a permanent magnet Zeeman slower with a heated window, but due to the inclusion of a 2D-MOT which deflects the atomic beam, the heated window and oven will not be visible to atoms in the main chamber. Finally, the specified pressure after the oven of $<5 \times 10^{-9}$ Torr is significantly better than SrII's, and the pressure farther away from the oven after the 2D-MOT and before the differential pump tube should be even better. Needless to say, a ready built system like this was hard to pass up.

For the main chamber, we examined purchasing another Kimball physics chamber or designing our own custom system. However, as our main goal was to achieve long vacuum lifetimes, stock parts did not seem like an option. Getting enough pumping into the main chamber meant either sacrificing one of the large viewports or adding a custom piece onto one of the large viewports. As we did not want to lose any of our large viewports, a fully custom solution seemed the smart choice. After examining many groups chambers we found a design that was both practical and proven. Aviv Keshet's thesis from Wolfgang Ketterle's group wonderfully outlined the construction of a chamber with a large (many 100s of L/s) conductance path to vacuum pumps, and was nice enough to offer CAD files so large modifications could be included in his chamber's design [107]. As can be seen in figure 6.2 the main attraction to this chamber design is the large rectangular path intersecting both the main chamber and auxiliary pumping chambers. Furthermore, viewports surrounding the chamber could be placed in approximately 30 degree intervals meeting our optical access requirements as well.

The main chamber construction was a fairly complicated job that required professional vacuum machinists. After talking with a few companies, we proceeded with Sharon Vacuum who had previously built Aviv Keshet's chamber as well. Appendix B includes the CAD drawings of the chamber. The final chamber was built with 304 stainless steel and electropolished by Sharon 

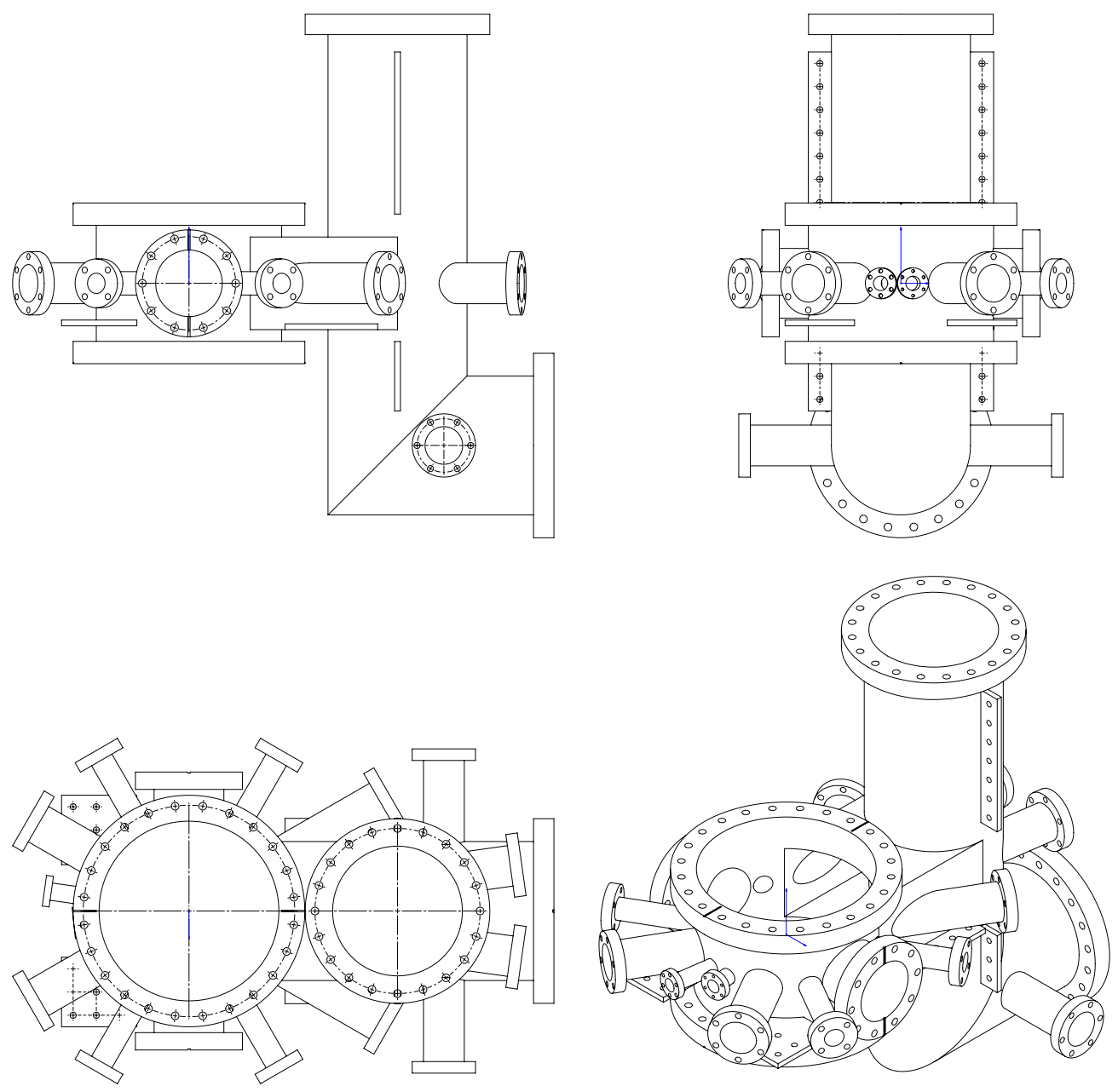

Figure 6.2: The SrIII Main Chamber. The vacuum system was built with most ports giving a 30 degree angle between neighboring ports, allowing for 30, 60, 90, or 120 degree angles all accessible. The chamber was constructed by Sharon Vacuum where it was electropolished and leak tested. After verifying the leak test at JILA, it was shipped to Jefferson Lab where it was vacuum baked at $400 \mathrm{C}$ for 100 hours. Following this, it was sputter coated for 100 Hours with $40 \mathrm{~mA}$ going through the TiZrV wires, and 30 Hours at $150 \mathrm{~mA}$. All blanks used in this process were specially machined with jack screws for prying off stuck blanks, furthermore belleville washers were used with all the screws to help alleviate metal fusing at these high temperatures. More details about the main chamber can be found in the engineering diagrams in Appendix B. 
Vacuum. The bottom of the L-shaped auxiliary pumping chamber in other designs had held a $75 \mathrm{~L} / \mathrm{s}$ ion pump while the top of the $\mathrm{L}$ held a titanium sublimation pump, final quoted pressure values for the main chamber seemed to put the main chamber pressure at low $10^{-11}$ Torr. Rather than just strictly adapt this chamber design, it seemed worthwhile to examine where it might be improved. Although equations for gas conductance in the molecular flow regime are valid only under large lengths, $L$, compared to tube diameters, $D$. We can nonetheless use the equation as a guide, $C=3.64 \frac{\pi}{4} \frac{D^{3}}{L} \sqrt{\frac{T}{M}}$, where $D$ and $L$ are in $\mathrm{cm}, T$ is the temperature of the gas in $\mathrm{K}, M$ is the relative atomic mass of the gas, and $\mathrm{C}$ is computed in units of $\mathrm{L} / \mathrm{s}$. Using this equation, the pathway to the pump would seem to have many hundreds of L/s conductance of hydrogen, so increasing the pump speed would directly benefit the ultimate pressure in the main chamber.

However, purchasing larger and larger ion pumps requires both heavier pumps, putting physical strain on the new apparatus, and larger pump magnets possibly interfering with the magnetic field at the atoms. Furthermore, pumping speeds of ion pumps decrease at lower vacuum levels causing diminishing gains. Instead, we purchased a pump from SAES Getters based on Non-Evaporable Getter (NEG) technology where residual gases are sorbed onto porous sintered vanadium zirconium iron $(\mathrm{VZrFe})$ discs. This technology has the benefits of being compact and requiring no power after activation, however it is completely incapable of pumping away certain gas species and the inclusion of a small ion pump is usually required. SrIII includes a $300 \mathrm{~L} / \mathrm{s}$ combination NEG-ion pump. All the NEG material is hidden by the elbow of the L-shape from a titanium sublimation pump (TSP), which also has no line of sight to any vacuum viewports. Both of these pumps excel at pumping hydrogen gas, which is the predominant residual gas in clean XHV systems.

An additional pumping mechanism has been included in the main chamber with the help of staff members at Jefferson Labs. Thanks to the efforts of Philip Adderley, Marcy Stutzman, and Matt Poelker our chamber walls were coated with additional NEG material. This material, titanium zirconium vanadium (TiZrV), although a less effective pump, has a significantly reduced activation temperature of $\sim 180{ }^{\circ} \mathrm{C}$. This allows us to activate the NEG material on surfaces very close to coated vacuum viewports that are specified to only go up to $200{ }^{\circ} \mathrm{C}$. Although commercially 
manufactured coatings of this type by SAES quote pumping speeds fairly comparable to sputtered titanium from a TSP, our coating is expected to perform not as well. However, the coating itself also acts as a buffer between the steel and the vacuum lowering the outgassing rate of the steel chamber. All of these pumps combined with their different activation temperatures and properties make for a fairly complicated pumping procedure from atmospheric pressure all the way down to the system's ultimate base pressure.

\subsubsection{The Pumpdown Procedure}

Many NEG materials can be overwhelmed by large gas loads, and might require reactivation of the NEG material to achieve high pumping speeds. In many instances reactivation does not just more deeply sorb the material but rather pushes it back out into the vacuum, so reactivation should nominally be done in the presence of a turbo-molecular pump ${ }^{3}$. However, since reactivation or sputtering of titanium temporarily increases the gas load, these procedures should be performed in a manner where they do not interfere greatly with each other, neither procedure overwhelming the pumping benefits of the other. In fact, careful operation and preparation of a TSP pump should

allow short, quick sputtering that should only raise the overall pressure to low $10^{-8}$ or high $10^{-9}$ Torr as read by a nude ion gauge.

Given the lowest sorption capacities listed for our NEG pump of $\sim 1$ Torr.Liter of carbon monoxide, it would take approximately 1 million seconds, or 11.5 days, of pumping a $10^{-8}$ vacuum to sorb these capacities of CO. Therefore, the goal is to correctly prime the TSP to allow for clean sputtering, that will not overwhelm the NEG pump, nor will it overwhelm the NEG coating. Many of the ideas presented in this list are pulled together from the manuals for the NEG ion pump, the TSP, and the turbo-molecular pump. In addition to the instructions in this list, it is important that all maximum temperature requirements for the viewports, pump magnet, electrical cords, turbo pump flange, and any other components be followed exactly.

\footnotetext{
${ }^{3}$ Some quantities are sorbed deep into the material, so reactivation still does lessen the overall gas load in the long term.
} 
(1) After engaging the roughing pump, turn on the turbo pump while the system is still at room temperature.

(2) Make sure the cables, metal container, and pump magnets are removed from the ion pump. Heat the ion pump to a safe baking temperature $\left(\max 350{ }^{\circ} \mathrm{C}\right)$, and bake out for at least a day or two.

(3) Cool the ion pump to room temperature, temporarily reassemble the ion pump magnets and prime the ion pump at least once. This needs to be done before ever activating any of the NEG elements of the chamber. Remove pump magnets, etc. and prepare for a full system bake.

(4) Slowly heat the entire vacuum system to a temperature well below the TiZrV activation temperature, say $\sim 150{ }^{\circ} \mathrm{C}$, place the NEG pump into bakeout/conditioning mode which heats the pump without activating it. Additionally, run $\sim 25$ A through each TSP filament to keep them hot.

(5) After the background pressure is well below $10^{-8}$ Torr, begin to clean each TSP filament according the the instructions in the manual. Run 37 to $42 \mathrm{~A}$ through each filament for approximately 1 minute each. Between filament outgassings continually run 25 Amps through each filament to keep them hot.

(6) Continue baking the chamber at a temperature well below the TiZrV activation temperature, keep performing the TSP filament outgassing until the pressure rise from outgassing any filament peaks in the high $10^{-9}$. Once this is accomplished, the filaments should be clean enough for use post-bake ${ }^{4}$.

(7) Once pumping down at this temperature starts to slow down, raise the temperature to above the activation temperature of $\operatorname{TiZrV}\left(180^{\circ} \mathrm{C}\right)$. This temperature must be kept for

\footnotetext{
${ }^{4}$ Other groups in JILA have recorded $49 \mathrm{~A}$ for 7 minutes raising pressure to $3 \times 10^{-8}$, and pressure falling once the current has been shut off. Depending on the performance of the TSP filaments, this could also be a goal during the bake.
} 
at least 24 hours or longer to ensure activation of the TiZrV.

(8) If at the end of this high temperature activation phase, the pressure is mid or low $10^{-9}$ Torr proceed with activation of the VZrFe NEG pump. This will greatly increase the gas load.

(9) After the activation of the VZrFe NEG pump is over, begin cooling the system down. If the pressure was too high before to activate the NEG pump, activate the NEG pump once the system has cooled. Remember to cool all components of the system including the TSP filaments.

(10) After the system is room temperature, reassemble the ion pump magnets and turn the ion pump back on.

(11) Close the all-metal gate valve, and turn off the turbo. If after the gate valve has been closed for a few hours the pressure is not low enough, fire the now clean TSP pump.

\subsection{Recessed Viewports for spectroscopic resolution}

In the spirit of adding additional capabilities to SrIII that neither SrI nor SrII had, the new system will include recessed top and bottom viewports. Not only do these recessed viewports allow imaging lenses to be placed significantly closer to the atoms, they also will allow us to attain incredibly large magnetic field gradients up to 1000 Gauss/cm. This will allow spectroscopic resolution of single pancakes of atoms in our optical lattice, a technique that could be especially interesting for looking at atomic noise correlations [34]. Construction of these recessed viewports was accomplished by the United Kingdom Atomic Energy Authority (UKAEA) ${ }^{5}$. In addition to their expertise in constructing these specialized UHV windows, they have extensive experience building these windows in a piecewise manner. They first braze the glass onto the bottom disc, before connecting the disk to a CF flange with a recessed wall. This allows us to have the front

\footnotetext{
5 These viewports were designed to be similar to those found in Wolfgang Ketterle's apparatus, with some minor changes for creating an intra-vacuum mounting system for future experiments.
} 
and back of the glass viewports AR coated before the completed "bucket" makes coating them impossible due to shadowing. Figure 6.3 shows what these recessed viewports will look like, and Appendix B holds mechanical drawings of the viewport. Special thanks should be given to Tim Darby, an engineer at UKAEA.

The ability to perform experiments on individual pancakes, or to address individual atoms in a 3D lattice, will further the development of precision optical control of strontium atoms. It is my hope that with a combination of magnetic field gradients and easily attainable resolution requirements for the clock laser, SrIII could one day be considered a quantum gas microscope in its own manner.

\subsection{Optical Access}

While in experiments with alkali atoms a great emphasis is placed on both magnetic field performance and the system's ability to deliver RF to the atoms, for alkaline earth atoms given the myriad number of ways light and matter interactions are introduced to the atoms the system's ability to deliver clean light to the atomic sample is incredibly important. While the use of commercial-off-the-shelf (COTS) chambers allows quick and easy design choices, not only have we been disappointed with the viewport angular placement, we have also found that not all viewports are usuable once mounting structures and magnetic coils have been attached. By going custom with sufficient planning of viewport placement we have higher hopes for the future of the SrIII main chamber. Anti-reflection (AR) coating was also of great concern to us, especially as SrII's successful demonstration of an extra-vacuum cavity hinged upon our viewports' AR coating.

Previous work in SrII, had in fact suffered from insufficient AR coating planning. While multiband coatings were implemented that covered the ${ }^{1} S_{0}$ to ${ }^{1} P_{1},{ }^{3} P_{1},{ }^{3} P_{0}$ transitions, and various magic wavelengths, no attention had been paid to widely available dipole trapping wavelengths in the NIR. In fact, our current viewports reflect $15 \%$ of $1064 \mathrm{~nm}$ light per surface, reducing the single pass power delivered to the atoms by at least 30\%. Not only would SrIII require multiband coatings for ports that would deliver clock light, but broadband coatings would need to be found that would 
not limit any future laser light choices. While many companies were contacted, American Photonics offered the best multiband coating given our requirements. Their quoted specifications are given in Figure 6.4. While many companies competed for our multiband coatings, the search for a truly broadband coating that could stretch to include $461 \mathrm{~nm}$ and $1064 \mathrm{~nm}$, was fairly difficult. In the end, TAKOS came through for us offering a fairly impressive broadband coating (see Figure 6.5).

Careful selection of which viewports to coat with which coating was performed with an eye towards future endeavors. Figure 6.6 shows a top-down view of the SrIII apparatus, with colorcoding indicating the coating for each viewport. The top, bottom, and large side viewports (ports 4a and $4 \mathrm{~b}$ ) were chosen to have the multiband coating. This would allow for superior absorption imaging, fluorescence detection, the possibility of magic wavelength enhancement cavities, and clock laser launching. Port pairs 6 and 2 were also chosen because their 60 degree angle would possibly be useful in studying dipole-dipole interactions. All other ports are coated with broadband coatings, such that the use of $688 \mathrm{~nm}$, anti-magic wavelength lasers, or any other visible or NIR wavelength would never be limited by the viewports themselves.

Having briefly discussed some of the features of SrIII, we will now examine how SrIII will support investigations into two different experiments.

\subsubsection{Dipole-Dipole Interactions}

The study of long-range interactions is a goal for much of atomic physics, whether the interaction is mediated through optical cavities, dipolar interactions, or even coulomb interactions. Precision control of long-range interactions might lead to a better understanding of strongly correlated materials, or might be used to entangle spatially separated qubits. While proposals exist for achieving dipole interaction energies in strontium gases comparable to polar molecule interaction energies, these proposals have not yet been implemented [108]. However, our work with atomic clocks has taught us a great deal about the power of using clock shifts themselves to measure incredibly small interaction energies [109], which could unexpectedly turn into a tool for creating quantum correlated states when other single particle dephasing has been suppressed. 
The atoms themselves when placed in a coherent superposition between the ground state ${ }^{1} S_{0}$ and the electronically excited ${ }^{3} \mathrm{P}_{0}$ state do have a tiny dipole moment of approximately $40 \mu$ Debye. By changing the geometric position of an array of atoms in a 3D lattice, one can create spatially coherent arrays of dipoles that will resonantly shift the clock transition [110]. Observing these shifts will require high phase space density samples of atoms, loaded into lattices with very specific intersection angles. The SrIII apparatus has been designed with a majority of viewports being separated by 30 degrees so that achieving the $\sim 64.8$ degree resonant angle is possible. Furthermore, creating cubic lattices which will suppress this shift will also be possible with viewports set 90 degrees from each other, making a differential measurement possible. The final smoking gun will be the atomic number scaling of this shift which should go like $N^{2 / 3}$. After many years of work, these shifts are finally within our reach of observing as they should be high $10^{-18}$ in magnitude for reasonable atom numbers.

Not only will this experiment need to be performed with deeply degenerate samples of atoms, necessitating long vacuum lifetimes, the optical access requirements were not met with SrII.

\subsubsection{Spin-Orbit Physics in Alkaline Earth Atoms}

The study of artificial gauge fields in cold atoms to simulate the behavior of charged particles moving around in a magnetic field is a fast growing topic around the world $[111,112,113,114]$. By coherently addressing atoms and imparting phase shifts as they traverse closed paths in either the bulk of the trap or around specific lattice sites, an analog to the Aharnov-Bohm phase of enclosed magnetic flux is created [115]. Creating these effects on the level of single particles has been achieved, however heating either via scattering field-dressing lasers or other less well understood mechanisms has slowed the progress of this field. To study interesting effects, like the Hofstedter Butterfly transition or to create topologically non-trivial states, requires not only engineering single particle dispersion curves that are flat and highly degenerate, but creating a system where the interparticle interactions become the dominant energy scale while still obeying the dressed states that have been imposed on the atoms. 
The connection between dressed-state interactions and some of the first proposed density shift mechanisms for OLCs (based on laser inhomogeneity induced interactions) was even commented upon by Prof. Jing Zhang on a visit to our group [33]. In fact, the creation of velocity and position dependent Rabi coupling to our clock state has in the past been used for mundane diagnostics such as Doppler temperature scans. While some of the original proposals for spin-orbit coupling utilized the narrowness of our clock state due to its lack of scattering, excited state loss due to inelastic collisions might put a damper on these proposals. However, multi-clock-photon coupling to different ground state nuclear spin states might remain a saving grace for implementing many of these proposals.

While options for spin-orbit coupling for atoms in shallow lattices abound, focusing on the experiments in the bulk also show off the benefits of working with alkaline earth atoms. Creating a dressing field that increases the flux of a fictitious field that scales favorably is not particularly easy. The first implementations of spin orbit coupling could only scale the flux with a single lengthscale of the cloud. Achieving high fictitious flux increases the number of vortices decreasing the filling factor $\left(N / N_{v}\right)$ and bringing the sample closer to the behavior of a superfluid electron gas, where the particles find it energetically favorable to form composite particles with the vortices present in the system [116]. By intersecting multiple dressing beams with specific detunings and angles one can however create an optical flux lattice [117]. These lattices will present an ever increasing amount of flux for closed paths in $2 \mathrm{D}$, scaling with area and not just length or width.

Creating these optical flux lattices again requires significant optical flexibility that SrII did not have. For example, the proposal in Ref. [118] required multiple beams to intersect a 2D pancake of atoms at 120 degrees from each other in the pancake plane. Furthermore, achieving meaningful correlated atom states requires an incredibly cold and dense atomic sample. While many of the proposals might not work out due to inelastic loss, or low interparticle interaction energies in strontium, I believe SrIII will have the necessary tools and the experience of using clock lasers to coherently address atoms and push the field of spin-orbit physics in an as of yet unexplored direction. 


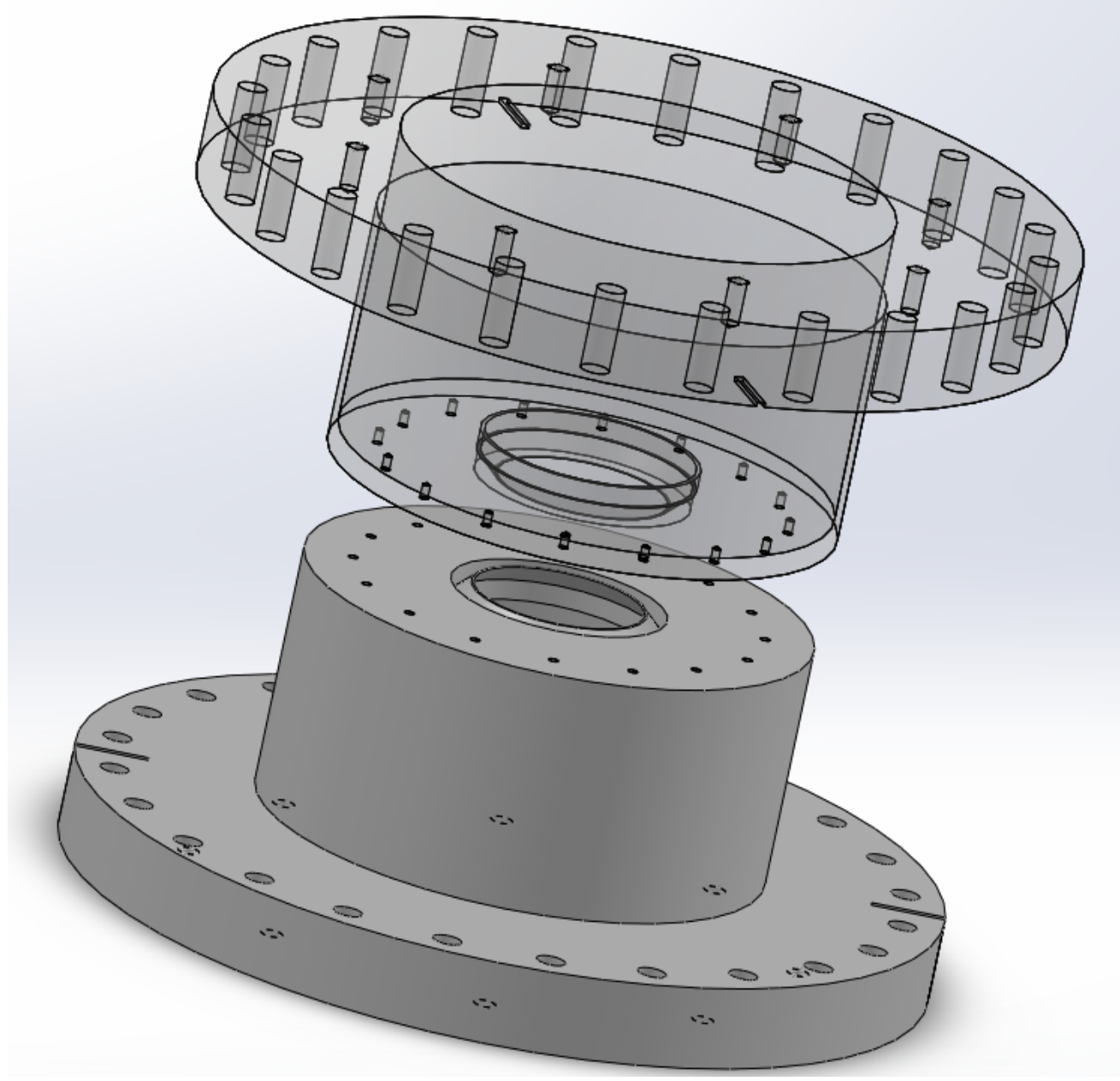

Figure 6.3: SrIII Recessed Viewports. The top viewport is depicted as a transparent wireframe for clarity. UKAEA will construct these viewports, first delivering the bottom of the bucket with the brazed window so it can be coated and then reattached to the rest of the bucket. More details about the bucket window can be found in the engineering diagrams in Appendix B. 


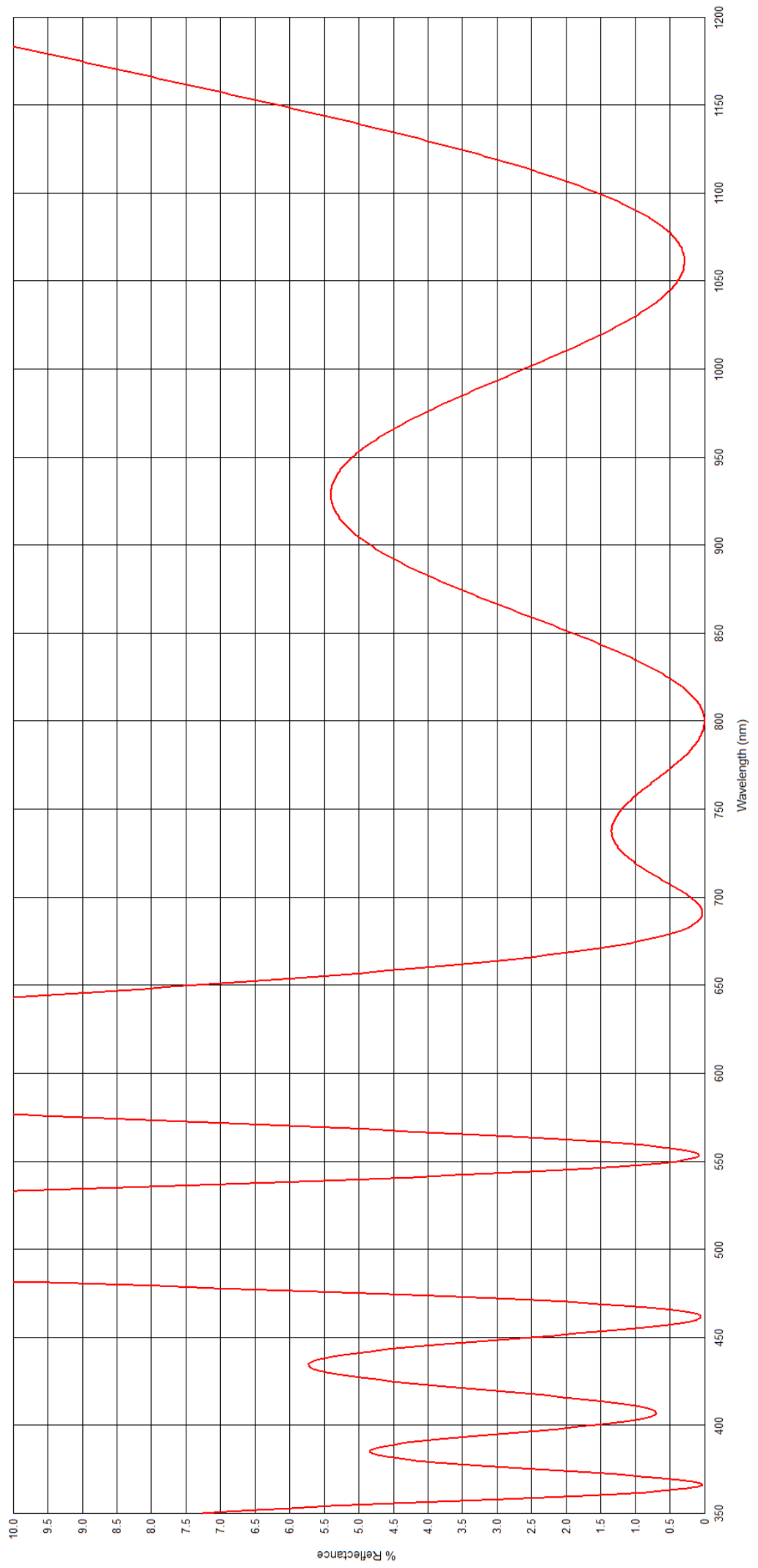

Figure 6.4: SrIII Multiband Coating from American Photonics. Special attention was paid to performance at $689 \mathrm{~nm}$ and $813 \mathrm{~nm}$. Coating performance at both $461 \mathrm{~nm}$ and $1064 \mathrm{~nm}$ was considered a secondary goal. 


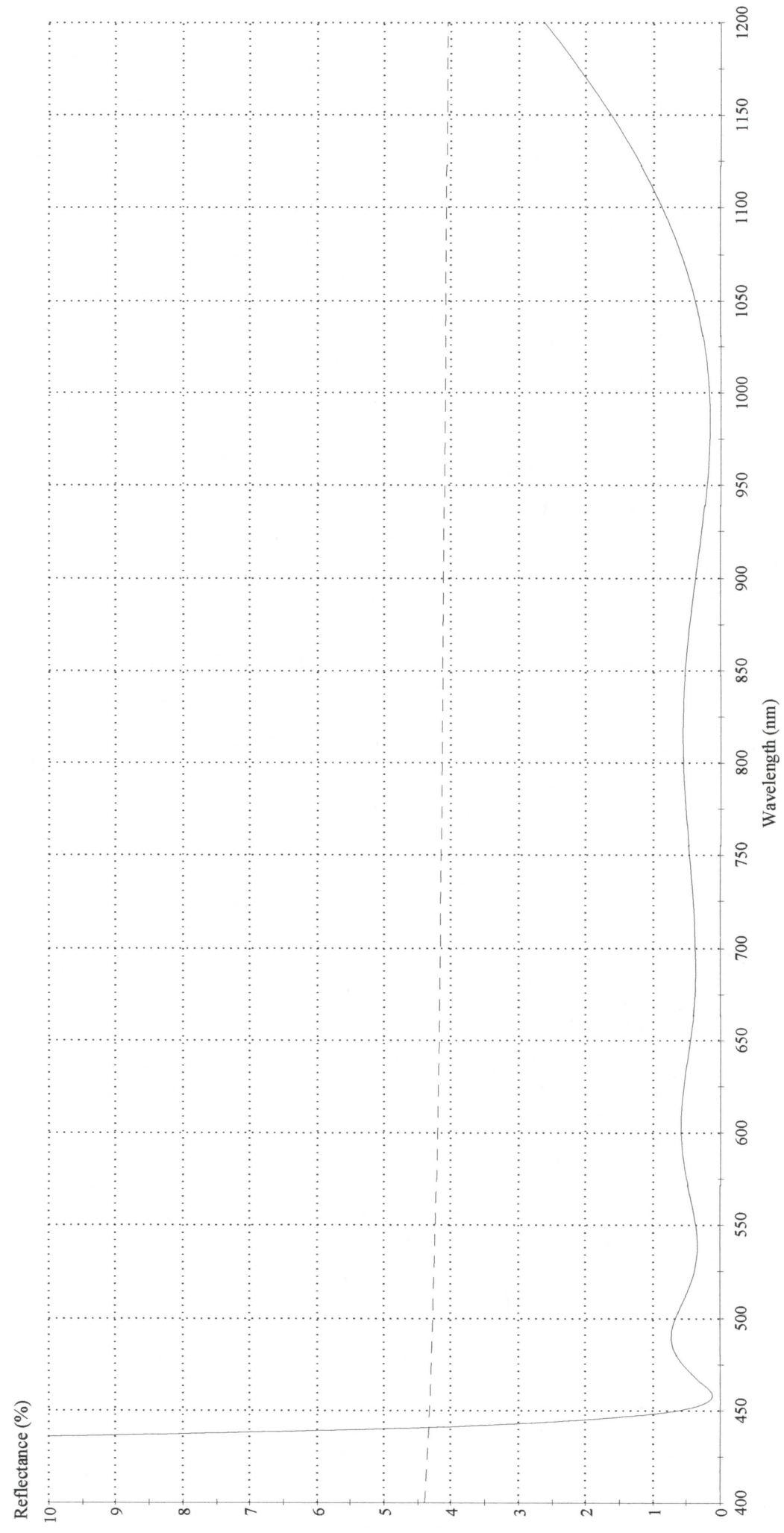

Figure 6.5: SrIII Broadband Coating from TAKOS. The solid line is the expected coating performance, while the dashed line is uncoated BK-7 glass. We wanted a coating that could be used for most of the visible and NIR, including more exotic wavelengths like the anti-magic wavelengths in the $650 \mathrm{~nm}$ region. 


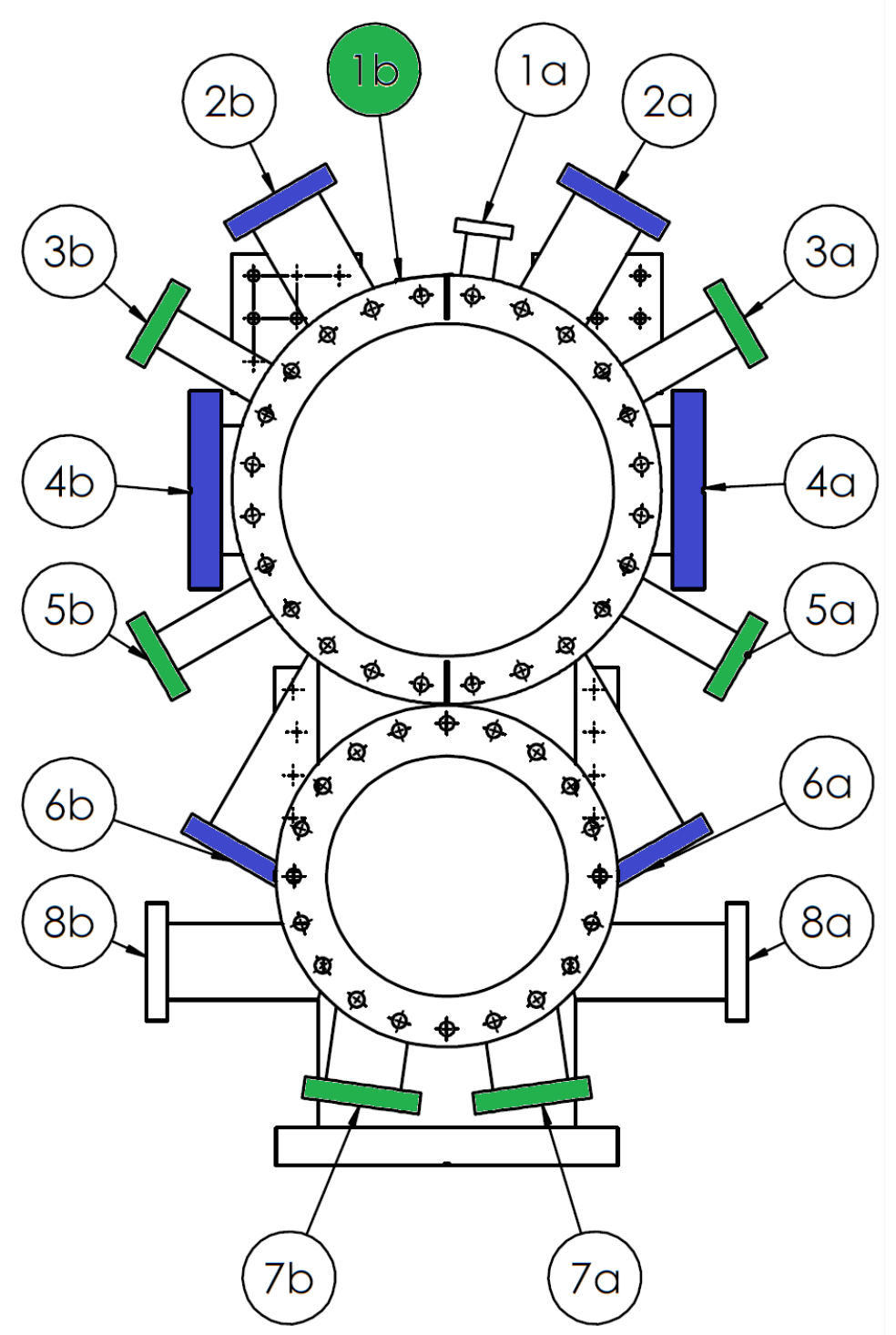

Figure 6.6: SrIII coating layout. The blue colored viewports (including the top and bottom bucket windows) are using the multiband coating. The green shaded viewports are coated with the broadband antireflection coating. 


\section{Chapter 7}

\section{Summary \& Outlook}

SrIII will no doubt push the boundaries of what is possible in a cold atomic gas machine. By combining the techniques the Ye group has honed in the frequency metrology business with a successful quantum gas apparatus, the next few years should prove very interesting [119]. But what does the future hold for atomic clocks? Let me first state unequivocally, I believe there is no end to accuracy or stability of clocks in sight. Whether or not we call future super-precise frequency metrology devices 'clocks' or 'quantum sensors', I believe that there are still decades of advances to be made.

In the field of ion clocks, at least three new contenders will emerge to battle the now triumphant optical lattice clocks. The first contender will be the $\mathrm{Yb}$ ion octupole transition clock. This extremely narrow transition offers the ability to push ion clock stability to the absolute limit of the stability of the optical local oscillator's stability. Because these clocks are still quantum projection noise limited with one ion, interrogation times will need to be incredibly long to achieve this. The second contender is the nuclear transition ${ }^{229} \mathrm{Th}$ ion clock. This transition, once it is found, is immune to many of the systematics associated with clocks as its nuclear nature shields it considerably from external perturbations [120]. Furthermore, it's nuclear nature allows for the possibility of embedding this isotope directly into a solid creating a crystal clock [121]. However, finding the exact frequency of this transition might require the use of novel technologies like an XUV comb or the Advanced Light Source at Lawrence Berkeley National Lab. [122, 123]. And

finally, other more accessible transitions in highly charged ions are also better shielded from ex- 
ternal perturbations and offer promising routes to systematic uncertainties of $10^{-19}[124]$. While OLCs might have got the upper hand for now, it would be a mistake to count out ion clocks in the running for redefinition of the second.

The high stability of neutral lattice clocks will continue to increase, as new materials and processes are tried to obtain better and better laser local oscillators. While our most recent results were driven by our new $40 \mathrm{~cm}$ long ULE cavity [62], a new round of results will appear resulting from cryogenically cooled silicon cavities [125] and custom crystalline Bragg mirrors [126]. Along the way more active laser technologies have been theorized and at least principally demonstrated that replace the phase memory of cavity mirrors with that of atomic dipoles creating incredibly narrow frequency sources $[127,128]$. It has been noted that frequency metrology improves by a factor of 10 with every decade [129] and so far I would say we are more than well on track.

But what of strontium? The blackbody radiation shift will remain its biggest obstacle. While modulation of the environment temperature coupled with clock comparisons might be a long term viable option, single system performance will continue to be limited. Neither temperature sensors nor small, cold enclosures will allow strontium to dip to far below $1 \times 10^{-18}[22,88]$. It may be that a combination of the two technologies will suffice. Instead, I suspect that for strontium to reach deep into fractional uncertainties of $10^{-19}$ or below, significant work will be needed to completely enclose the strontium cloud in a cold environment. Whether via a specially built cryostat chamber with light delivered by fiber or, as I have joked many times, an industrial freezer keeping the entire chamber at a chilly $187 \mathrm{~K}$, the next factor of 10 of systematic uncertainty will need a low temperature trapping environment.

The field of optical lattice clocks is rich though, and the methods pioneered in strontium by us and other groups around the world will one day be employed in a variety of species. A Yb clock using our temperature sensor procedure would have a 2 times smaller BBR uncertainty than ours, a Hg clock 50 times smaller. The high stability that these clocks can offer will for the forseeable future continue to be a selling point for their use, even if in the end it is as a flywheel technology used much like a hydrogen maser. This first triumph after more than a decade of research does not 
mark the beginning of the end of optical lattice clocks, but maybe the end of the beginning. 


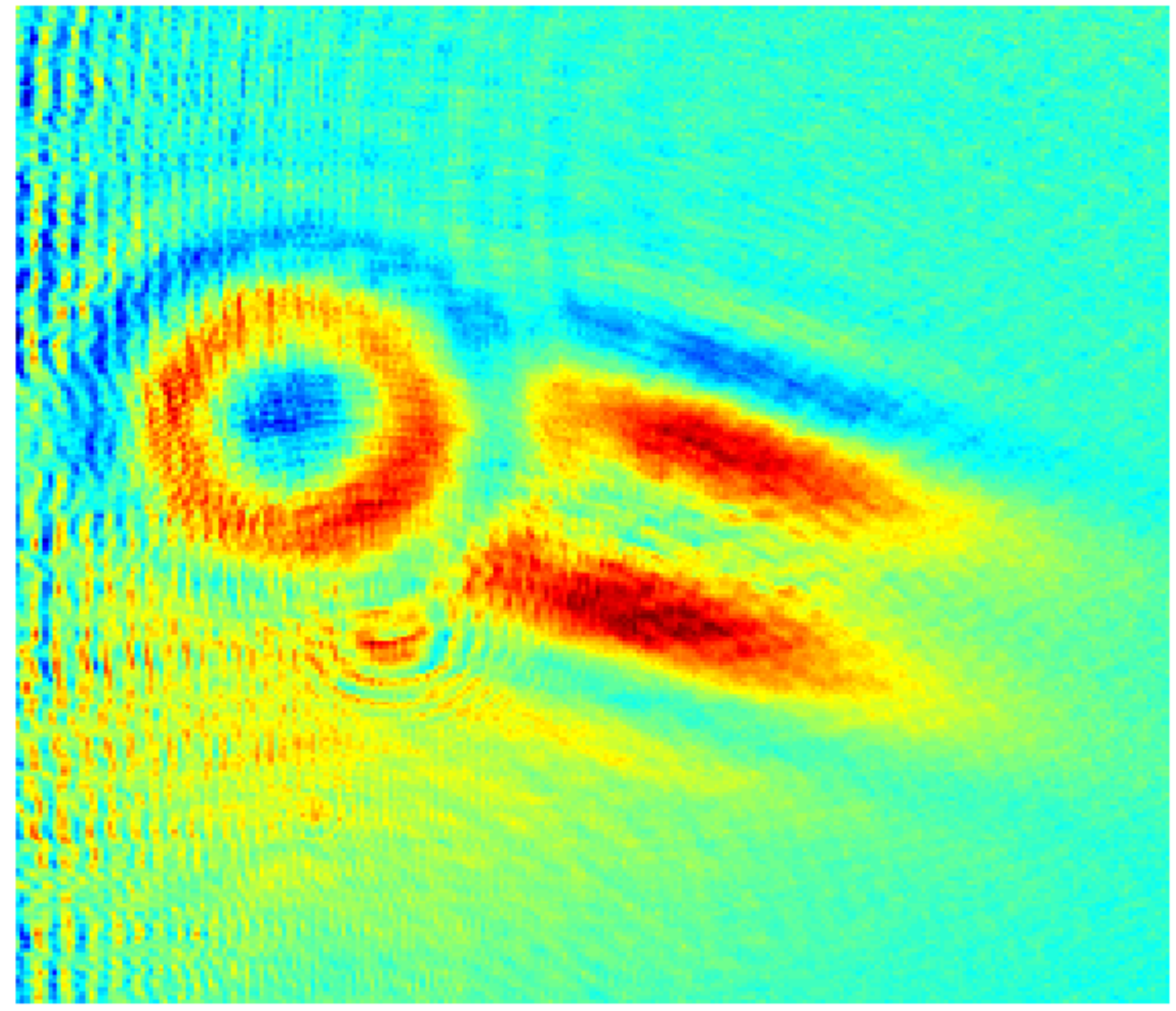

Figure 7.1: Red MOT absorption image of strontium atoms incorrectly aligned through the imaging optics. Boldly going where no optical lattice clock has gone before. 


\section{Bibliography}

[1] Gretchen K Campbell, Andrew D Ludlow, Sebastian Blatt, Jan W Thomsen, Michael J Martin, Marcio H G de Miranda, Tanya Zelevinsky, Martin M Boyd, Jun Ye, Scott A Diddams, Thomas P Heavner, Thomas E Parker, and Steven R Jefferts. The absolute frequency of the

${ }^{87}$ Sr optical clock transition. Metrologia, 45(5):539-548, October 2008.

[2] Andrew Ludlow. The Strontium Optical Lattice Clock: Optical Spectroscopy with Sub-Hertz Accuracy. PhD Thesis. University of Colorado, 2008.

[3] R Wynands and S Weyers. Atomic fountain clocks. Metrologia, 42(3):S64-S79, June 2005.

[4] T P Heavner, S R Jefferts, E A Donley, J H Shirley, and T E Parker. NIST-F1: recent improvements and accuracy evaluations. Metrologia, 42(5):411-422, October 2005.

[5] Krzysztof Szymaniec, Sang Eon Park, Giuseppe Marra, and Witold Chaupczak. First accuracy evaluation of the NPL-CsF2 primary frequency standard. Metrologia, 47(4):363-376, August 2010.

[6] V Gerginov, N Nemitz, S Weyers, R Schröder, D Griebsch, and R Wynands. Uncertainty evaluation of the caesium fountain clock PTB-CSF2. Metrologia, 47(1):65-79, February 2010.

[7] F Levi, D Calonico, L Lorini, and A Godone. IEN-CsF1 primary frequency standard at INRIM: accuracy evaluation and TAI calibrations. Metrologia, 43(6):545-555, December 2006 .

[8] Thomas P Heavner, Elizabeth A Donley, Filippo Levi, Giovanni Costanzo, Thomas E Parker, Jon H Shirley, Neil Ashby, Stephan Barlow, and S R Jefferts. First accuracy evaluation of NIST-F2. Metrologia, 51(3):174-182, June 2014.

[9] DW Allan, N Ashby, and CC Hodge. The science of timekeeping. 1997.

[10] L. Essen and J. V. L. Parry. An Atomic Standard of Frequency and Time Interval: A Cæsium Resonator. Nature, 176(4476):280-282, August 1955.

[11] H. Goldenberg, D. Kleppner, and N. Ramsey. Atomic Hydrogen Maser. Physical Review Letters, 5(8):361-362, October 1960.

[12] W. Itano, J. Bergquist, J. Bollinger, J. Gilligan, D. Heinzen, F. Moore, M. Raizen, and D. Wineland. Quantum projection noise: Population fluctuations in two-level systems. Physical Review A, 47(5):3554-3570, May 1993. 
[13] G. Santarelli, Ph. Laurent, P. Lemonde, A. Clairon, A. Mann, S. Chang, A. Luiten, and C. Salomon. Quantum Projection Noise in an Atomic Fountain: A High Stability Cesium Frequency Standard. Physical Review Letters, 82(23):4619-4622, June 1999.

[14] Mark Kasevich, Erling Riis, Steven Chu, and Ralph DeVoe. rf spectroscopy in an atomic fountain. Physical Review Letters, 63(6):612-615, August 1989.

[15] A Clairon, C Salomon, S Guellati, and W. D Phillips. Ramsey Resonance in a Zacharias Fountain. Europhysics Letters (EPL), 16(2):165-170, September 1991.

[16] Thomas E Parker. Invited review article: The uncertainty in the realization and dissemination of the SI second from a systems point of view. The Review of scientific instruments, 83(2):021102, March 2012.

[17] Thomas E Parker. Long-term comparison of caesium fountain primary frequency standards. Metrologia, 47(1):1-10, February 2010.

[18] David J. Wineland. Nobel Lecture: Superposition, entanglement, and raising Schrödingers cat. Rev. Mod. Phys., 85(3):1103-1114, July 2013.

[19] F. Diedrich, J. Bergquist, Wayne Itano, and D. Wineland. Laser Cooling to the Zero-Point Energy of Motion. Physical Review Letters, 62(4):403-406, January 1989.

[20] J. L. Hall, M. Zhu, and P. Buch. Prospects for using laser-prepared atomic fountains for optical frequency standards applications. Journal of the Optical Society of America B, 6(11):2194, November 1989.

[21] C. W. Chou, D. B. Hume, J. C. J. Koelemeij, D. J. Wineland, and T. Rosenband. Frequency Comparison of Two High-Accuracy Al ${ }^{+}$Optical Clocks. Phys. Rev. Lett., 104(7):070802, February 2010.

[22] B J Bloom, T L Nicholson, J R Williams, S L Campbell, M Bishof, X Zhang, W Zhang, S L Bromley, and J Ye. An optical lattice clock with accuracy and stability at the $10^{-18}$ level. Nature, 506(7486):71-5, February 2014.

[23] Jocelyne Guéna, Peter Rosenbusch, Philippe Laurent, Michel Abgrall, Daniele Rovera, Giorgio Santarelli, Michael E Tobar, Sébastien Bize, and André Clairon. Demonstration of a dual alkali $\mathrm{Rb} / \mathrm{Cs}$ fountain clock. IEEE transactions on ultrasonics, ferroelectrics, and frequency control, 57(3):647-53, March 2010.

[24] Martin M Boyd, Tanya Zelevinsky, Andrew D Ludlow, Seth M Foreman, Sebastian Blatt, Tetsuya Ido, and Jun Ye. Optical atomic coherence at the 1-second time scale. Science, 314(5804):1430-1433, December 2006.

[25] Michael Bishof, Xibo Zhang, Michael J. Martin, and Jun Ye. Optical spectrum analyzer with quantum limited noise floor. Phys. Rev. Lett., 111:093604, March 2013.

[26] Stephan Falke, Nathan Lemke, Christian Grebing, Burghard Lipphardt, Stefan Weyers, Vladislav Gerginov, Nils Huntemann, Christian Hagemann, Ali Al-Masoudi, Sebastian Häfner, Stefan Vogt, Uwe Sterr, and Christian Lisdat. A strontium lattice clock with 3 $\times 10^{-17}$ inaccuracy and its frequency. ArXiv:1312.3419, page 20, December 2013 . 
[27] R. Le Targat, L. Lorini, Y. Le Coq, M. Zawada, J. Guéna, M. Abgrall, M. Gurov, P. Rosenbusch, D. G. Rovera, B. Nagórny, R. Gartman, P. G. Westergaard, M. E. Tobar, M. Lours, G. Santarelli, A. Clairon, S. Bize, P. Laurent, P. Lemonde, and J. Lodewyck. Experimental realization of an optical second with strontium lattice clocks. Nat. Comm., July 2013.

[28] Masao Takamoto, Tetsushi Takano, and Hidetoshi Katori. Frequency comparison of optical lattice clocks beyond the Dick limit. Nat. Photonics, 5(5):288-292, April 2011.

[29] H. Hachisu, M. Fujieda, S. Nagano, T. Gotoh, A. Nogami, T. Ido, St. Falke, N. Huntemann, C. Grebing, B. Lipphardt, Ch. Lisdat, and D. Piester. Direct comparison of optical lattice clocks with an intercontinental baseline of $9000 \mathrm{~km}$. ArXiv:1403.6285, March 2014.

[30] Daisuke Akamatsu, Masami Yasuda, Hajime Inaba, Kazumoto Hosaka, Takehiko Tanabe, Atsushi Onae, and Feng-Lei Hong. Frequency ratio measurement of ${ }^{171} \mathrm{Yb}$ and ${ }^{87} \mathrm{Sr}$ optical lattice clocks. Optics express, 22(7):7898-905, April 2014.

[31] David Leibrandt, Michael Thorpe, Chin-Wen Chou, Tara Fortier, Scott Diddams, and Till Rosenband. Absolute and Relative Stability of an Optical Frequency Reference Based on Spectral Hole Burning in $\mathrm{Eu}^{3+}: \mathrm{Y}_{2} \mathrm{SiO}_{5}$. Physical Review Letters, 111(23):237402, December 2013.

[32] Hidetoshi Katori. Laser cooling of strontium atoms toward quantum degeneracy. In AIP Conference Proceedings, volume 551, pages 382-396. AIP, January 2001.

[33] G K Campbell, M M Boyd, J W Thomsen, M J Martin, S Blatt, M D Swallows, T L Nicholson, T Fortier, C W Oates, S A Diddams, N D Lemke, P Naidon, P Julienne, Jun Ye, and A D Ludlow. Probing interactions between ultracold fermions. Science, 324(5925):360-363, 2009.

[34] M. J. Martin, M. Bishof, M. D. Swallows, X. Zhang, C. Benko, J. Von-Stecher, A. V. Gorshkov, A. M. Rey, and J. Ye. A Quantum Many-Body Spin System in an Optical Lattice Clock. Science, 341(6146):632-636, August 2013.

[35] P. G. Westergaard, J. Lodewyck, L. Lorini, A. Lecallier, E. A. Burt, M. Zawada, J. Millo, and P. Lemonde. Lattice-Induced Frequency Shifts in Sr Optical Lattice Clocks at the $10^{-17}$ Level. Phys. Rev. Lett., 106(21):210801, May 2011.

[36] Hidetoshi Katori. Optical lattice clocks and quantum metrology. Nature Photonics, 5(4):203210, April 2011.

[37] N. Huntemann, M. Okhapkin, B. Lipphardt, S. Weyers, Chr. Tamm, and E. Peik. HighAccuracy Optical Clock Based on the Octupole Transition in ${ }^{171} \mathrm{Yb}^{+}$. Phys. Rev. Lett., 108(9):090801, February 2012.

[38] S. Blatt, A. D. Ludlow, G. K. Campbell, J. W. Thomsen, T. Zelevinsky, M. M. Boyd, J. Ye, M. Takamoto, F.-L. Hong, H. Katori, and V. V. Flambaum. New Limits on Coupling of Fundamental Constants to Gravity Using ${ }^{87} \mathrm{Sr}$ Optical Lattice Clocks. Phys. Rev. Lett., 100(14):2-5, April 2008.

[39] S. N. Lea. Limits to time variation of fundamental constants from comparisons of atomic frequency standards. The European Physical Journal Special Topics, 163(1):37-53, November 2008 . 
[40] Jonathan Weintroub. A submillimeter VLBI array. Journal of Physics: Conference Series, 131:012047, October 2008.

[41] C W Chou, D B Hume, T Rosenband, and D J Wineland. Optical clocks and relativity. Science, 329(5999):1630-1633, September 2010.

[42] V. V. Flambaum. Variation of the Fundamental Constants: Theory and Observations. International Journal of Modern Physics A, 22(27):4937-4950, October 2007.

[43] A. Derevianko and M. Pospelov. Hunting for topological dark matter with atomic clocks. ArXiv:1311.1244, page 5, November 2013.

[44] X. Zhang, M. Bishof, S. L. Bromley, C. V. Kraus, M. S. Safronova, P. Zoller, A. M. Rey, and J. Ye. Spectroscopic observation of SU(N)-symmetric interactions in Sr orbital magnetism. ArXiv:1403.2964, March 2014.

[45] Hidetoshi Katori, Tetsuya Ido, Yoshitomo Isoya, and Makoto Kuwata-Gonokami. MagnetoOptical Trapping and Cooling of Strontium Atoms down to the Photon Recoil Temperature. Physical Review Letters, 82(6):1116-1119, February 1999.

[46] Martin Boyd, Andrew Ludlow, Sebastian Blatt, Seth Foreman, Tetsuya Ido, Tanya Zelevinsky, and Jun Ye. isup $i 87 i / \sup$ iSr Lattice Clock with Inaccuracy below 10isupi-15i/supi. Phys. Rev. Lett., 98(8):083002, February 2007.

[47] Xinye Xu, Thomas H. Loftus, John L. Hall, Alan Gallagher, and Jun Ye. Cooling and trapping of atomic strontium. Journal of the Optical Society of America B, 20(5):968, 2003.

[48] R. Le Targat, J.-J. Zondy, and P. Lemonde. 75\%-Efficiency blue generation from an intracavity PPKTP frequency doubler. Optics Communications, 247(4-6):471-481, March 2005.

[49] Yang Zhao, Qiang Wang, Fei Meng, Yige Lin, Shaokai Wang, Ye Li, Baike Lin, Shiying Cao, Jianping Cao, Zhanjun Fang, Tianchu Li, and Erjun Zang. High-finesse cavity external optical feedback DFB laser with hertz relative linewidth. Optics Letters, 37(22):4729, November 2012 .

[50] Tadashi Nishikawa, Akira Ozawa, Yoshiki Nishida, Masaki Asobe, Feng-Lei Hong, and Theodor W Hänsch. Efficient $494 \mathrm{~mW}$ sum-frequency generation of sodium resonance radiation at $589 \mathrm{~nm}$ by using a periodically poled $\mathrm{Zn}: \mathrm{LiNbO}_{3}$ ridge waveguide. Optics express, 17(20):17792-800, September 2009.

[51] Kevin S. Repasky, Amin R. Nehrir, Justin T. Hawthorne, Gregg W. Switzer, and John L. Carlsten. Extending the continuous tuning range of an external-cavity diode laser. Applied Optics, 45(35):9013, 2006.

[52] Sheng-wey Chiow, Quan Long, Christoph Vo, Holger Müller, and Steven Chu. Extendedcavity diode lasers with tracked resonances. Applied Optics, 46(33):7997, November 2007.

[53] M K Shaffer, G Ranjit, and C I Sukenik. Extended tuning of an injection-locked diode laser. Review of Scientific Instruments, 79(4):046102, April 2008. 
[54] Matthew Swallows, Michael Martin, Michael Bishof, Craig Benko, Yige Lin, Sebastian Blatt, Ana Maria Rey, and Jun Ye. Operating a ${ }^{87} \mathrm{Sr}$ optical lattice clock with high precision and at high density. IEEE transactions on ultrasonics, ferroelectrics, and frequency control, 59(3):416-25, March 2012.

[55] Jérôme Lodewyck, Michal Zawada, Luca Lorini, Mikhail Gurov, and Pierre Lemonde. Observation and cancellation of a perturbing dc stark shift in strontium optical lattice clocks. IEEE Trans. Ultrason. Ferroelectr. Freq. Control, 59(3):411-415, March 2012.

[56] Warren Nagourney. Quantum Electronics for Atomic Physics. Oxford University Press, Seattle, WA, 2010.

[57] Anthony E Siegman. Lasers. University Science Books, 1986.

[58] Eric D. Black. An introduction to Pound-Drever-Hall laser frequency stabilization. American Journal of Physics, 69(1):79, 2001.

[59] Richard W. Fox, Chris W. Oates, and Leo W. Hollberg. Stabilizing Diode Lasers to HighFinesse Cavities. In Experimental Methods in the Physical Sciences. Elsevier Science.

[60] M. Gehm, K. OHara, T. Savard, and J. Thomas. Dynamics of noise-induced heating in atom traps. Physical Review A, 58(5):3914-3921, November 1998.

[61] Wikipedia: Serial Peripheral Interface Bus.

[62] T. L. Nicholson, M. J. Martin, J. R. Williams, B. J. Bloom, M. Bishof, M. D. Swallows, S. L. Campbell, and J. Ye. Comparison of Two Independent Sr Optical Clocks with $1 \times 10^{-17}$ stability at 10³. Phys. Rev. Lett., 109(23):230801, December 2012.

[63] A D Ludlow, T Zelevinsky, G K Campbell, S Blatt, M M Boyd, M H G de Miranda, M J Martin, J W Thomsen, S M Foreman, Jun Ye, T M Fortier, J E Stalnaker, S A Diddams, Y Le Coq, Z W Barber, N Poli, N D Lemke, K M Beck, and C W Oates. Sr lattice clock at $1 \times 10^{-16}$ fractional uncertainty by remote optical evaluation with a Ca clock. Science, 319(5871):1805-1808, March 2008.

[64] Matthew D Swallows, Michael Bishof, Yige Lin, Sebastian Blatt, Michael J Martin, Ana Maria Rey, and Jun Ye. Suppression of collisional shifts in a strongly interacting lattice clock. Science, 331(6020):1043-1046, February 2011.

[65] N. D. Lemke, J. vonStecher, J. A. Sherman, A. M. Rey, C. W. Oates, and A. D. Ludlow. p-Wave Cold Collisions in an Optical Lattice Clock. Phys. Rev. Lett., 107(10):103902, August 2011.

[66] Michael J Martin. Quantum Metrology and Many-Body Physics : Pushing the Frontier of the Optical Lattice Clock by. PhD Thesis. University of Colorado, 2013.

[67] St Falke, H Schnatz, J S R Vellore Winfred, Th Middelmann, St Vogt, S Weyers, B Lipphardt, G Grosche, F Riehle, U Sterr, and Ch Lisdat. The ${ }^{87} \mathrm{Sr}$ optical frequency standard at PTB. Metrologia, 48(5):399-407, October 2011.

[68] T. L. Nicholson. Strontium Thesis. PhD Thesis. University of Colorado. 
[69] Rudolf Grimm, Matthias Weidemüller, and Yurii B. Ovchinnikov. Optical Dipole Traps for Neutral Atoms. Advances In Atomic, Molecular, and Optical Physics, 42:95-170, 2000.

[70] Anders Brusch, Rodolphe Le Targat, Xavier Baillard, Mathilde Fouché, and Pierre Lemonde. Hyperpolarizability Effects in a Sr Optical Lattice Clock. Phys. Rev. Lett., 96(10):103003, March 2006.

[71] A. Taichenachev, V. Yudin, V. Ovsiannikov, V. Palchikov, and C. Oates. Frequency Shifts in an Optical Lattice Clock Due to Magnetic-Dipole and Electric-Quadrupole Transitions. Physical Review Letters, 101(19):193601, November 2008.

[72] S. Blatt, J. W. Thomsen, G. K. Campbell, a. D. Ludlow, M. D. Swallows, M. J. Martin, M. M. Boyd, and Jun Ye. Rabi Spectroscopy and Excitation Inhomogeneity in a 1D Optical Lattice. Phys. Rev. A., 80:052703, 2009.

[73] Philip R. Bevington and D. Keith Robinson. Data Reduction and Error Analysis. McGrawHill, third edition, 2003.

[74] Thomas Middelmann, Stephan Falke, Christian Lisdat, and Uwe Sterr. High Accuracy Correction of Blackbody Radiation Shift in an Optical Lattice Clock. Phys. Rev. Lett., 109(26):263004, December 2012.

[75] Long-Sheng Ma, Peter Jungner, Jun Ye, and John L. Hall. Delivering the same optical frequency at two places: accurate cancellation of phase noise introduced by an optical fiber or other time-varying path. Optics Letters, 19(21):1777, November 1994.

[76] S. Falke, M. Misera, U. Sterr, and C. Lisdat. Delivering pulsed and phase stable light to atoms of an optical clock. Applied Physics B, 107(2):301-311, March 2012.

[77] J. R. Williams. Private communications.

[78] V. I. Yudin, A. V. Taichenachev, C. W. Oates, Z. W. Barber, N. D. Lemke, A. D. Ludlow, U. Sterr, Ch. Lisdat, and F. Riehle. Hyper-Ramsey spectroscopy of optical clock transitions. Physical Review A, 82(1):011804, July 2010.

[79] Chr. Tamm. Private communications.

[80] Nobuyasu Shiga, Ying Li, Hiroyuki Ito, Shigeo Nagano, Tetsuya Ido, Katarzyna Bielska, Ryszard Trawiski, and Roman Ciuryo. Buffer-gas-induced collision shift for the ${ }^{88} \mathrm{Sr}^{1} S_{0}-{ }^{3} P_{1}$ clock transition. Phys. Rev. A., 80(3):030501, September 2009.

[81] Kurt Gibble. Scattering of Cold-Atom Coherences by Hot Atoms: Frequency Shifts from Background-Gas Collisions. Physical Review Letters, 110(18):180802, May 2013.

[82] Robin Santra, Kevin Christ, and Chris Greene. Properties of metastable alkaline-earth-metal atoms calculated using an accurate effective core potential. Physical Review A, 69:042510, April 2004.

[83] M. S. Safronova, M. G. Kozlov, Dansha Jiang, Bindiya Arora, Charles W. Clark, U. I. Safronova, and W. R. Johnsonk. Blackbody radiation shifts and theoretical contributions to atomic clock research. In 2009 IEEE International Frequency Control Symposium Joint with the 22nd European Frequency and Time forum, pages 132-140. IEEE, April 2009. 
[84] J. A. Sherman, N. D. Lemke, N. Hinkley, M. Pizzocaro, R. W. Fox, A. D. Ludlow, and C. W. Oates. High-Accuracy Measurement of Atomic Polarizability in an Optical Lattice Clock. Physical Review Letters, 108(15):153002, April 2012.

[85] K. Beloy, J. A. Sherman, N. D. Lemke, N. Hinkley, C. W. Oates, and A. D. Ludlow. Determination of the $5 d 6 s^{3} D_{1}$ state lifetime and blackbody-radiation clock shift in Yb. Physical Review A, 86(5):051404, November 2012.

[86] John Farley and William Wing. Accurate calculation of dynamic Stark shifts and depopulation rates of Rydberg energy levels induced by blackbody radiation. Hydrogen, helium, and alkali-metal atoms. Physical Review A, 23(5):2397-2424, May 1981.

[87] M. S. Safronova, S. G. Porsev, U. I. Safronova, M. G. Kozlov, and Charles W. Clark. Blackbody-radiation shift in the Sr optical atomic clock. Phys. Rev. A., 87(1):012509, January 2013.

[88] Ichiro Ushijima, Masao Takamoto, Manoj Das, Takuya Ohkubo, and Hidetoshi Katori. Cryogenic optical lattice clocks with a relative frequency difference of $1 \times 10^{-18}$. ArXiv:1405.4071, May 2014.

[89] T Middelmann, C Lisdat, S Falke, J S R Vellore Winfred, F Riehle, and U Sterr. Tackling the Blackbody Shift in a Strontium Optical Lattice Clock. IEEE Trans. Instrum. Meas., 60(7):2550-2557, July 2011.

[90] Wayne Itano, L. Lewis, and D. Wineland. Shift of ${ }^{2} S_{1 / 2}$ hyperfine splittings due to blackbody radiation. Physical Review A, 25(2):1233-1235, February 1982.

[91] Masami Yasuda and Hidetoshi Katori. Lifetime Measurement of the ${ }^{3} P_{2}$ Metastable State of Strontium Atoms. Phys. Rev. Lett., 92(15):153004, April 2004.

[92] R J Chandos and R E Chandos. Radiometric properties of isothermal, diffuse wall cavity sources. Appl. Opt., 13(9):2142-2152, September 1974.

[93] Evaluation of measurement data - Guide to the expression of uncertainty in measurement. JCGM 100:2008, 2008.

[94] Tien-Tsin Wong, Wai-Shing Luk, and Pheng-Ann Heng. Sampling with Hammersley and Halton Points. Journal of Graphics Tools, 2(2):9-24, January 1997.

[95] A. G. McNamara. Semiconductor Diodes and Transistors as Electrical Thermometers. Rev. Sci. Instrum., 33(3):330-333, March 1962.

[96] A. H. Harvey, M. O. McLinden, and W. L. Tew. Thermodynamic analysis and experimental study of the effect of atmospheric pressure on the ice point. In Temperature: Its Measurement and Control in Science and Industry, Volume 8: Proceedings of the Ninth International Temperature Symposium, volume 1552, pages 221-226. AIP Publishing, September 2013.

[97] Martin W. Zwierlein. High-Temperature Superfluidity in an Ultracold Fermi Gas. PhD thesis, Massachusetts Institute of Technology, 2006.

[98] Simon Stellmer, Rudolf Grimm, and Florian Schreck. Detection and manipulation of nuclear spin states in fermionic strontium. Physical Review A, 84(4):043611, October 2011. 
[99] M. Bishof, M. J. Martin, M. D. Swallows, C. Benko, Y. Lin, G. Quéméner, A. M. Rey, and J. Ye. Inelastic collisions and density-dependent excitation suppression in a ${ }^{87} \mathrm{Sr}$ optical lattice clock. Physical Review A, 84(5):052716, November 2011.

[100] Shinya Kato, Seiji Sugawa, Kosuke Shibata, Ryuta Yamamoto, and Yoshiro Takahashi. Control of Resonant Interaction between Electronic Ground and Excited States. Physical Review Letters, 110(17):173201, April 2013.

[101] Simon Stellmer, Benjamin Pasquiou, Rudolf Grimm, and Florian Schreck. Laser Cooling to Quantum Degeneracy. Physical Review Letters, 110(26):263003, June 2013.

[102] Simon Stellmer, Rudolf Grimm, and Florian Schreck. Production of quantum-degenerate strontium gases. Physical Review A, 87(1):013611, January 2013.

[103] Sebastian Blatt. Ultracold Collisions and Fundamental Physics with Strontium. PhD Thesis. University of Colorado, 2011.

[104] Eric Wille. Preparation of an Optically Trapped Fermi-Fermi Mixture of Li6 and K40 Atoms and Characterization of the Interspecies Interactions by Feshbach Spectroscopy. PhD Thesis. University of Innsbruck, 2009.

[105] Ian R Hill, Yuri B Ovchinnikov, Elizabeth M Bridge, E Anne Curtis, and Patrick Gill. Zeeman slowers for strontium based on permanent magnets. Journal of Physics B: Atomic, Molecular and Optical Physics, 47(7):075006, April 2014.

[106] G. Reinaudi, C. B. Osborn, K. Bega, and T. Zelevinsky. Dynamically configurable and optimizable Zeeman slower using permanent magnets and servomotors. Journal of the Optical Society of America B, 29(4):729, March 2012.

[107] Aviv Keshet. A Next-Generation Apparatus for Lithium Optical Lattice Experiments. PhD thesis, Massachusetts Institute of Technology, 2012.

[108] B. Olmos, D. Yu, Y. Singh, F. Schreck, K. Bongs, and I. Lesanovsky. Long-Range Interacting Many-Body Systems with Alkaline-Earth-Metal Atoms. Physical Review Letters, 110(14):143602, April 2013.

[109] M. Bishof, Y. Lin, M. D. Swallows, A. V. Gorshkov, J. Ye, and A. M. Rey. Resolved Atomic Interaction Sidebands in an Optical Clock Transition. Physical Review Letters, 106(25):250801, June 2011.

[110] D. Chang, Jun Ye, and M. Lukin. Controlling dipole-dipole frequency shifts in a lattice-based optical atomic clock. Physical Review A, 69(2):023810, February 2004.

[111] Y-J Lin, R L Compton, K Jiménez-García, J V Porto, and I B Spielman. Synthetic magnetic fields for ultracold neutral atoms. Nature, 462(7273):628-32, December 2009.

[112] Pengjun Wang, Zeng-Qiang Yu, Zhengkun Fu, Jiao Miao, Lianghui Huang, Shijie Chai, Hui Zhai, and Jing Zhang. Spin-Orbit Coupled Degenerate Fermi Gases. Physical Review Letters, 109(9):095301, August 2012.

[113] Hirokazu Miyake, Georgios A. Siviloglou, Colin J. Kennedy, William Cody Burton, and Wolfgang Ketterle. Realizing the Harper Hamiltonian with Laser-Assisted Tunneling in Optical Lattices. Physical Review Letters, 111(18):185302, October 2013. 
[114] M. Aidelsburger, M. Atala, M. Lohse, J. T. Barreiro, B. Paredes, and I. Bloch. Realization of the Hofstadter Hamiltonian with Ultracold Atoms in Optical Lattices. Physical Review Letters, 111(18):185301, October 2013.

[115] Jean Dalibard, Fabrice Gerbier, Gediminas Juzelinas, and Patrik Öhberg. Colloquium: Artificial gauge potentials for neutral atoms. Reviews of Modern Physics, 83(4):1523-1543, November 2011.

[116] Nathan Gemelke, Edina Sarajlic, and Steven Chu. Rotating Few-body Atomic Systems in the Fractional Quantum Hall Regime. ArXiv:1007.2677, July 2010.

[117] N. R. Cooper. Optical Flux Lattices for Ultracold Atomic Gases. Physical Review Letters, 106(17):175301, April 2011.

[118] N. R. Cooper and J. Dalibard. Optical flux lattices for two-photon dressed states. EPL (Europhysics Letters), 95(6):66004, September 2011.

[119] K-K Ni, S Ospelkaus, M H G de Miranda, A Pe'er, B Neyenhuis, J J Zirbel, S Kotochigova, P S Julienne, D S Jin, and J Ye. A high phase-space-density gas of polar molecules. Science (New York, N.Y.), 322(5899):231-5, October 2008.

[120] C. J. Campbell, A. G. Radnaev, A. Kuzmich, V. A. Dzuba, V. V. Flambaum, and A. Derevianko. Single-Ion Nuclear Clock for Metrology at the 19th Decimal Place. Physical Review Letters, 108(12):120802, March 2012.

[121] G A Kazakov, A N Litvinov, V I Romanenko, L P Yatsenko, A V Romanenko, M Schreitl, G Winkler, and T Schumm. Performance of a ${ }^{229}$ Thorium solid-state nuclear clock. New Journal of Physics, 14(8):083019, August 2012.

[122] Arman Cingöz, Dylan C Yost, Thomas K Allison, Axel Ruehl, Martin E Fermann, Ingmar Hartl, and Jun Ye. Direct frequency comb spectroscopy in the extreme ultraviolet. Nature, 482(7383):68-71, February 2012.

[123] Markus P. Hehlen, Richard R. Greco, Wade G. Rellergert, Scott T. Sullivan, David DeMille, Robert A. Jackson, Eric R. Hudson, and Justin R. Torgerson. Optical spectroscopy of an atomic nucleus: Progress toward direct observation of the ${ }^{229}$ Th isomer transition. Journal of Luminescence, 133:91-95, January 2013.

[124] M. S. Safronova, V. A. Dzuba, V. V. Flambaum, U. I. Safronova, S. G. Porsev, and M. G. Kozlov. Highly-charged ions for atomic clocks, quantum information, and search for $\alpha$ variation. May 2014.

[125] T. Kessler, C. Hagemann, C. Grebing, T. Legero, U. Sterr, F. Riehle, M. J. Martin, L. Chen, and J. Ye. A sub-40-mHz-linewidth laser based on a silicon single-crystal optical cavity. Nat. Photonics, 6(10):687-692, September 2012.

[126] Garrett D. Cole, Wei Zhang, Michael J. Martin, Jun Ye, and Markus Aspelmeyer. Tenfold reduction of Brownian noise in high-reflectivity optical coatings. Nat. Photonics, 7(8):644650, July 2013.

[127] D. Meiser, Jun Ye, D. Carlson, and M. Holland. Prospects for a Millihertz-Linewidth Laser. Physical Review Letters, 102(16):163601, April 2009. 
[128] Justin G Bohnet, Zilong Chen, Joshua M Weiner, Dominic Meiser, Murray J Holland, and James K Thompson. A steady-state superradiant laser with less than one intracavity photon. Nature, 484(7392):78-81, April 2012.

[129] Christian J Bordé. Base units of the SI, fundamental constants and modern quantum physics. Phil. Trans. R. Soc. A, 363(1834):2177-2201, September 2005. 


\section{Appendix A}

\section{Single Purpose Fast Arduino Example}

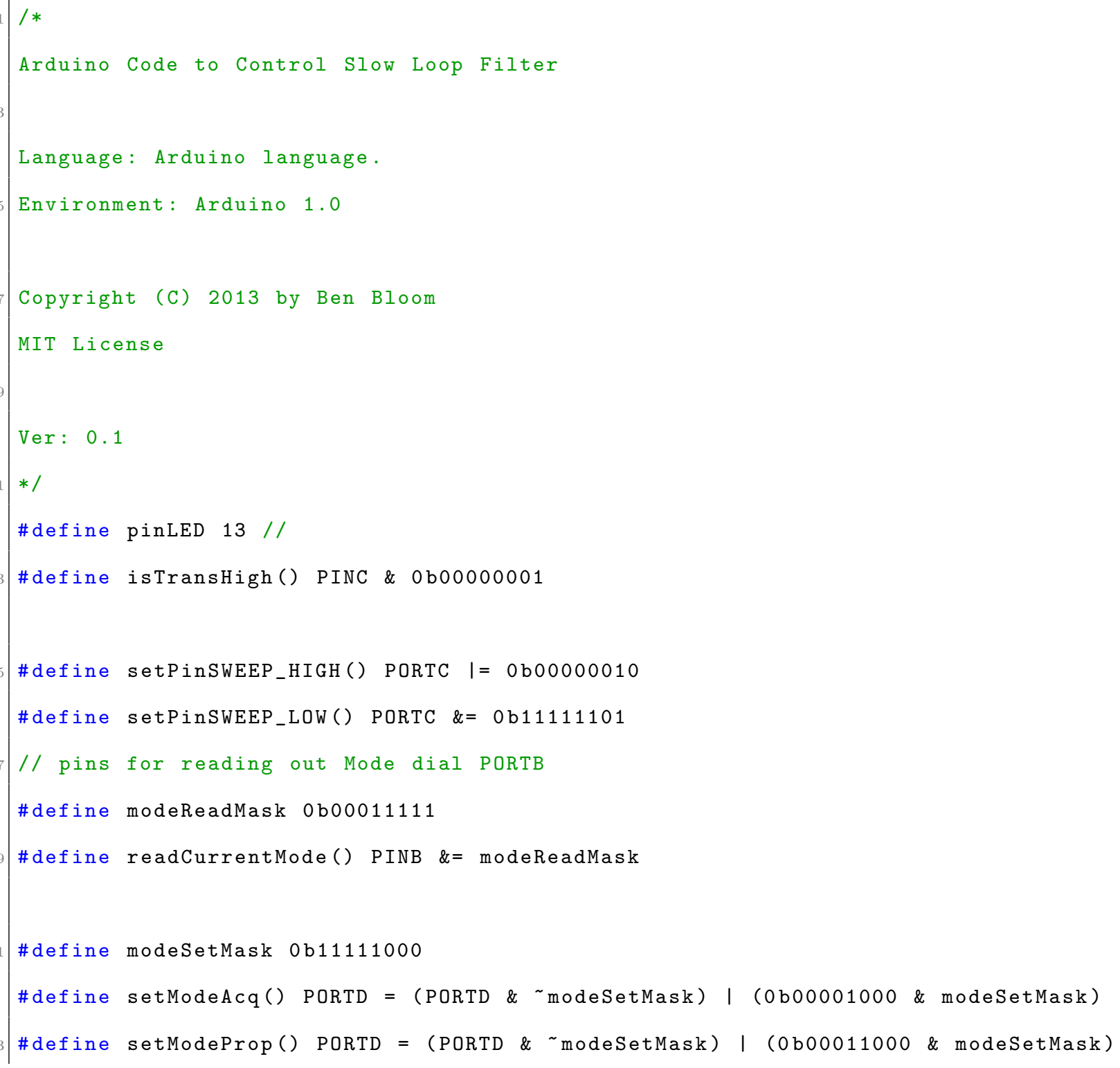




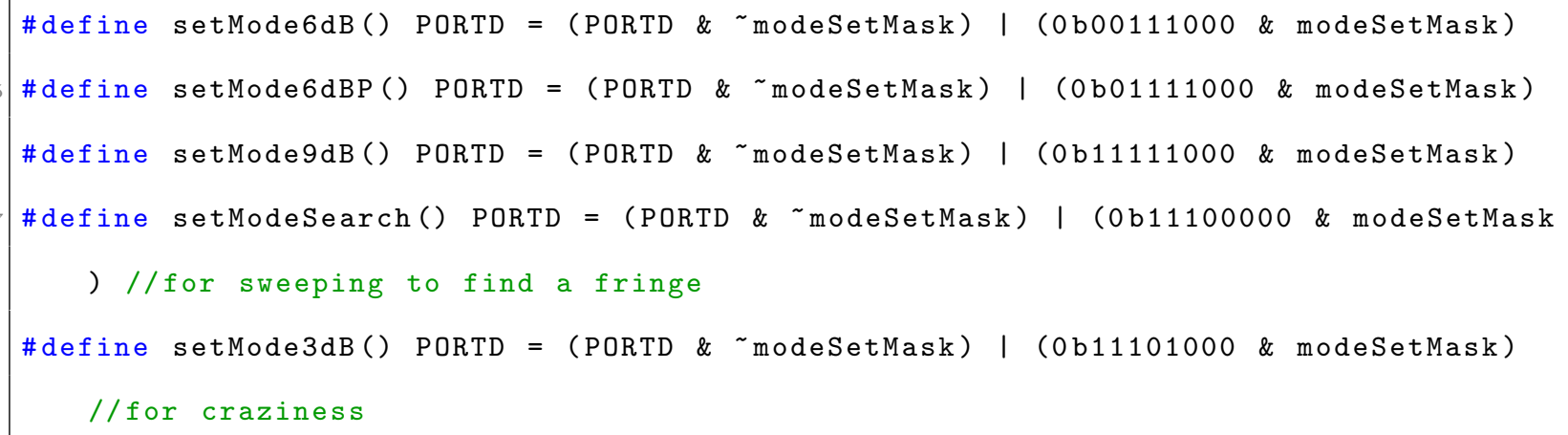




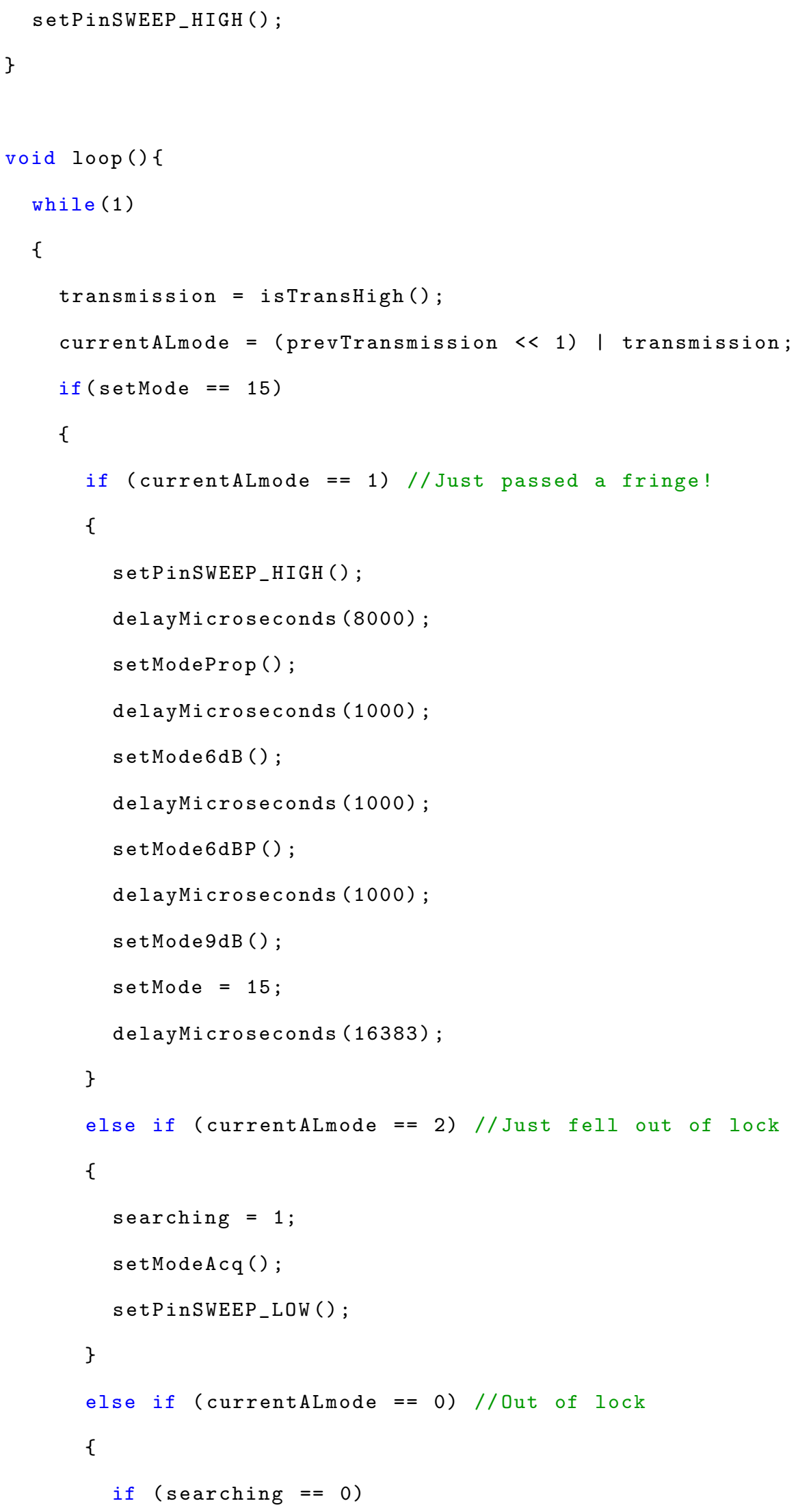




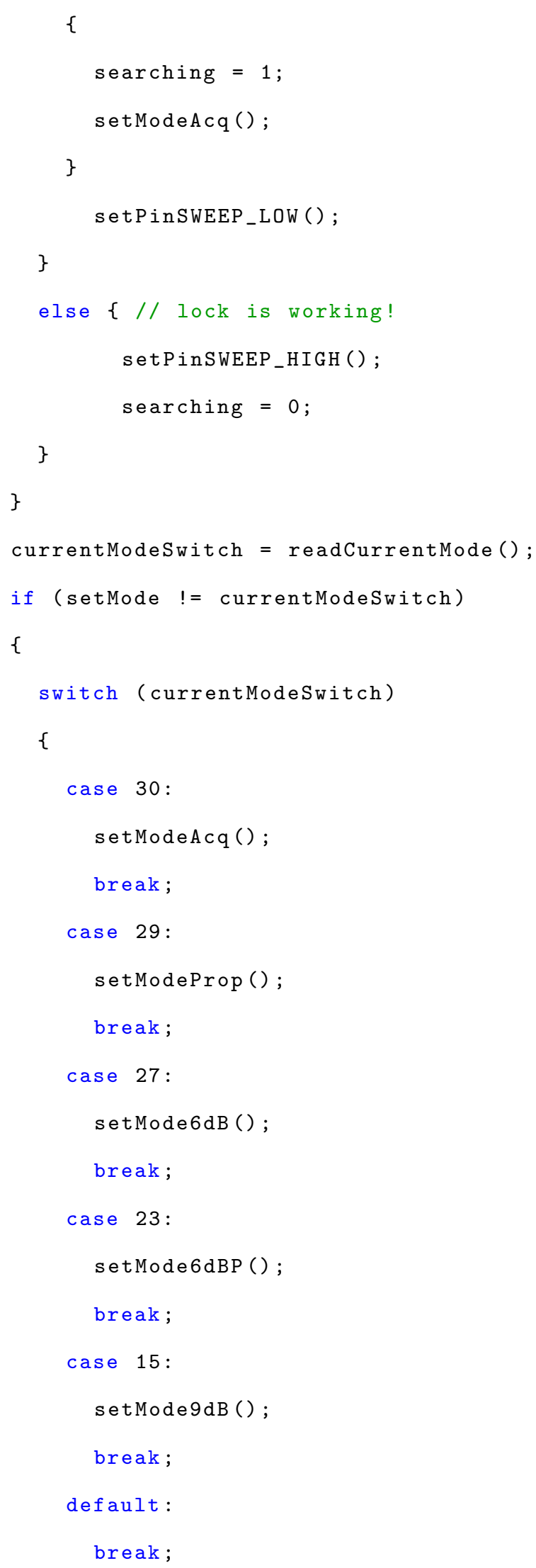




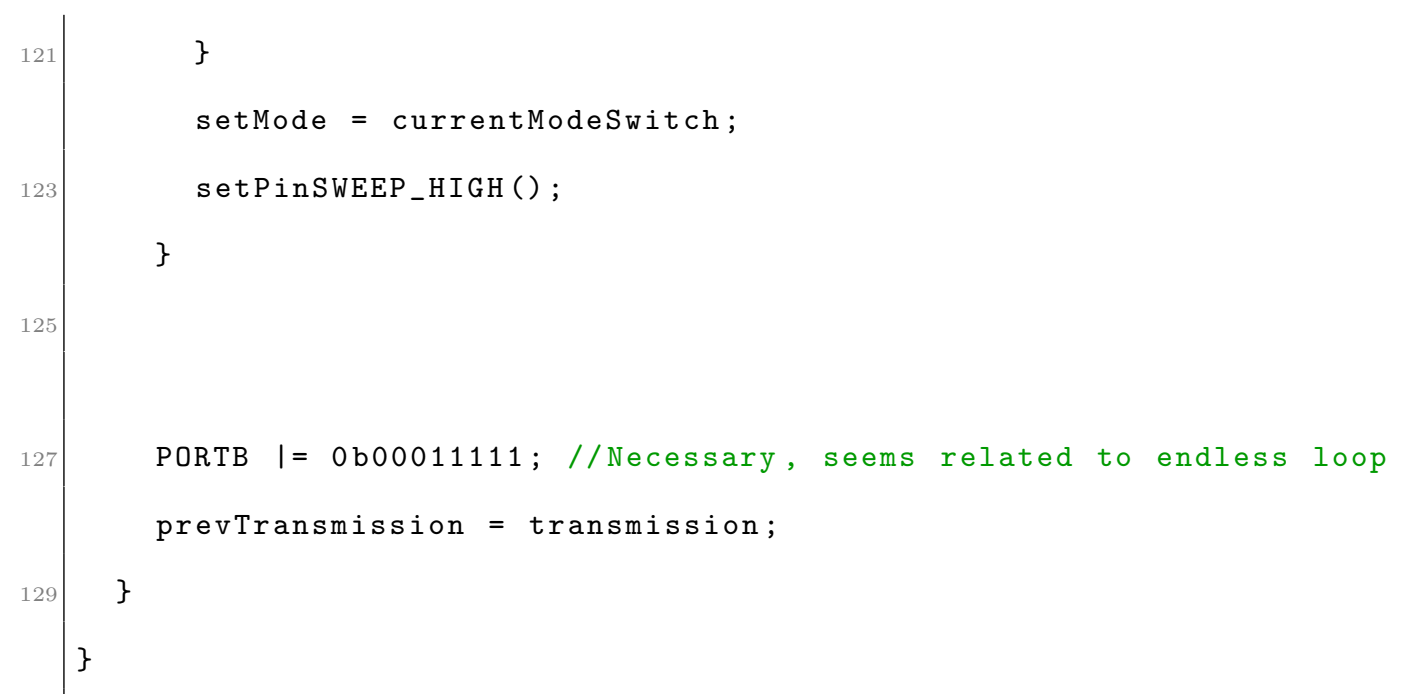

code/LoopFilterAutoLock.ino 


\section{Appendix B}

\section{Technical Drawings for SrIII Apparatus}

What follows are a collection of technical CAD drawings of the SrIII main chamber and the recessed viewports. 


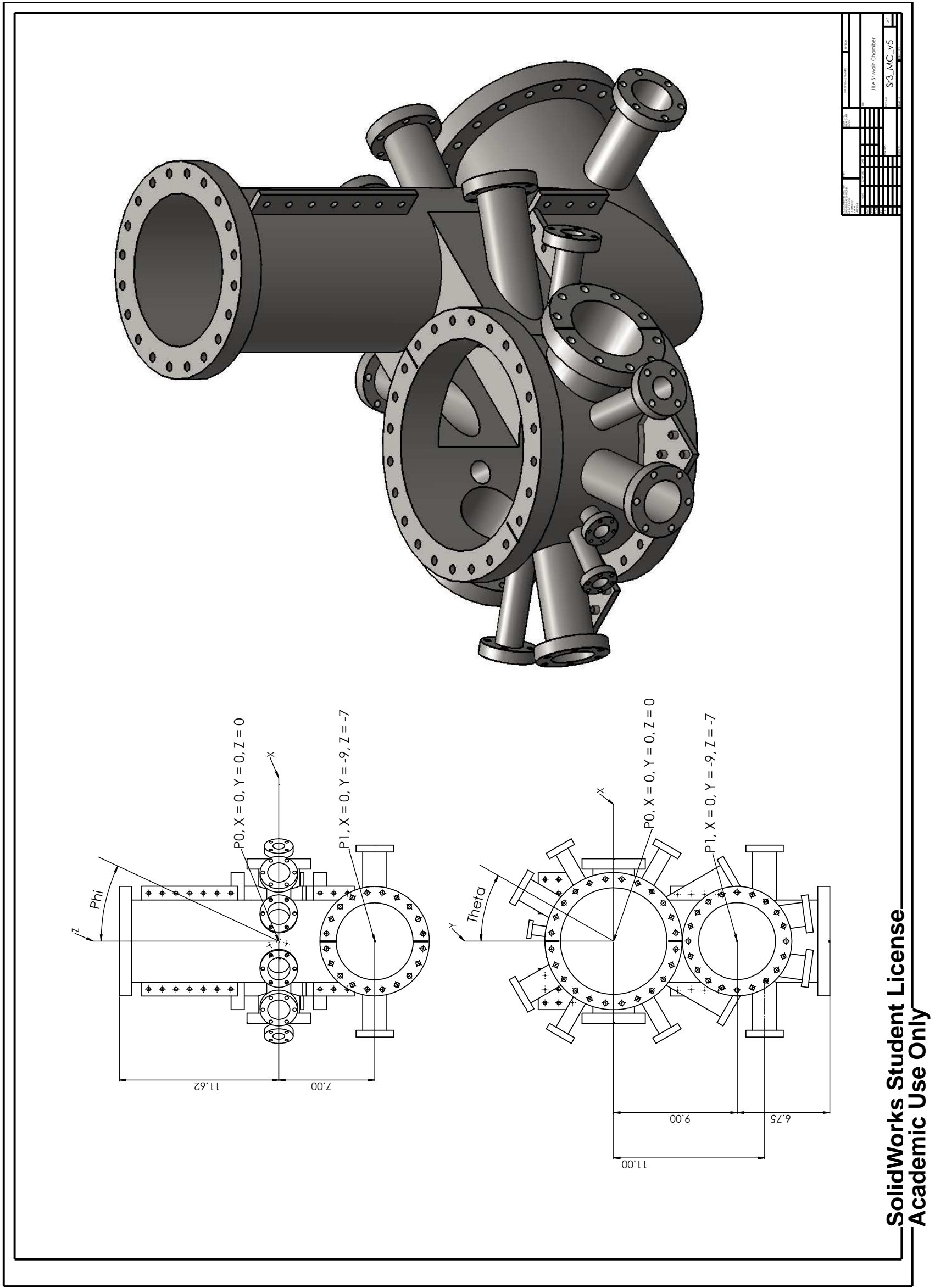



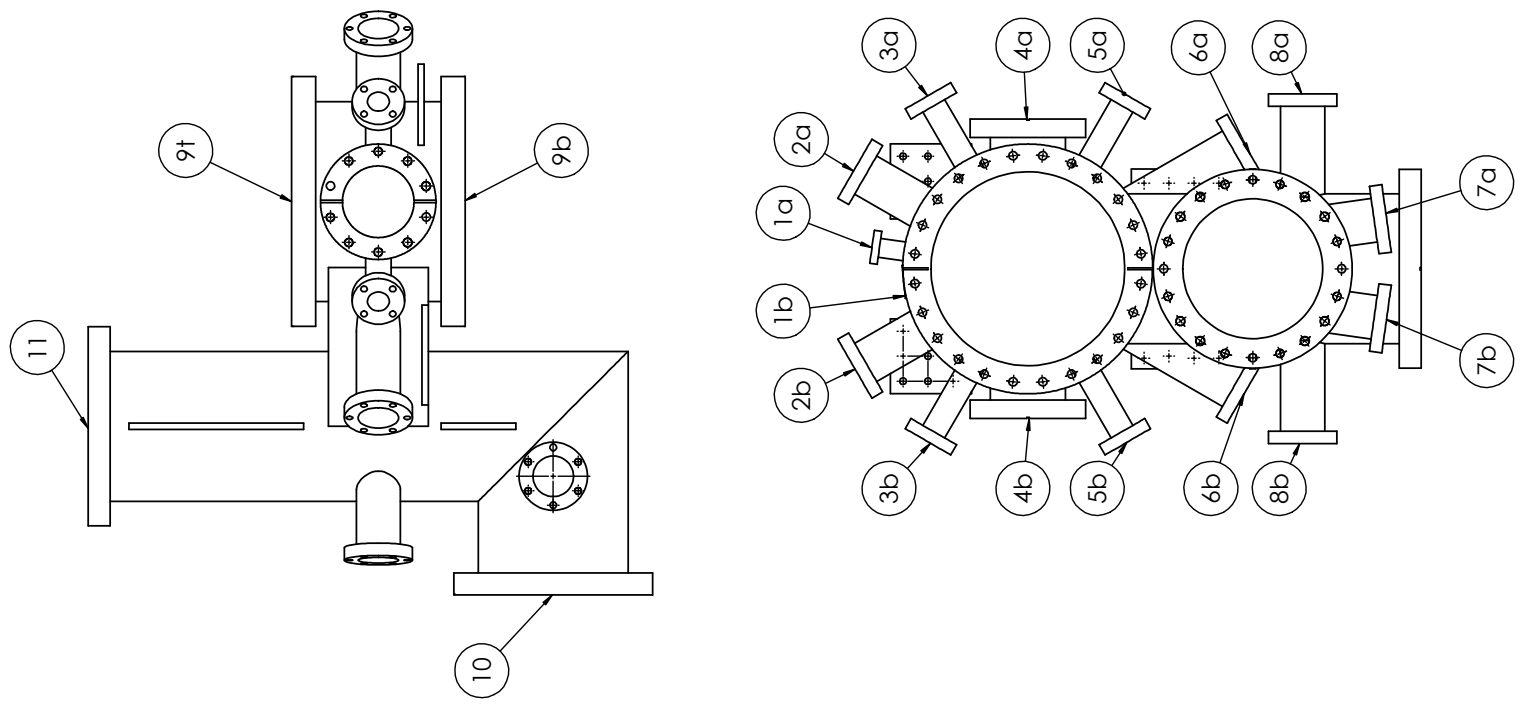

\begin{tabular}{|c|c|c|c|c|c|c|c|c|c|c|c|}
\hline & 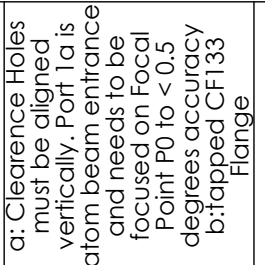 & & & & & & & 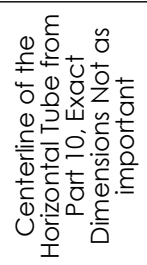 & 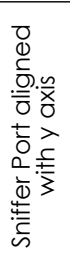 & 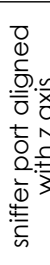 & \\
\hline$\overline{\bar{s}}$ & 음 & ㅇ & ০ & ㅇ & ㅇ & ㅇ & ㅇ & & $\stackrel{\circ}{\stackrel{\infty}{\oplus}}$ & ㅇ & 0 \\
\hline $\begin{array}{l}\frac{D}{0} \\
\frac{\Phi}{F}\end{array}$ & $\begin{array}{ll}\infty & \infty \\
\ddot{0} & \stackrel{1}{0} \\
\ddot{0}\end{array}$ & $\begin{array}{ll} & 0 \\
\text { er } & ? \\
0 \ddot{0}\end{array}$ & $\begin{array}{ll}\circ & \circ \\
0 & 0 \\
0 & 0\end{array}$ & $\begin{array}{ll}\circ & \circ \\
\circ & 0 \\
\ddot{0} & 1\end{array}$ & 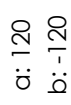 & 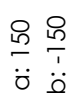 & 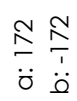 & & & $\stackrel{\infty}{\infty}$ & \\
\hline 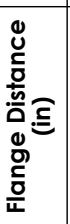 & 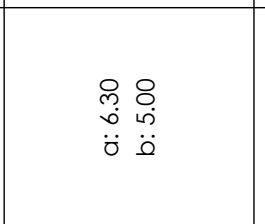 & $\begin{array}{l}8 \\
\infty \\
\infty\end{array}$ & $\underset{\infty}{\stackrel{\infty}{\infty}}$ & ষ্. & $\begin{array}{l}8 \\
\infty\end{array}$ & 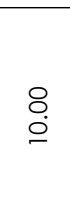 & 品 & 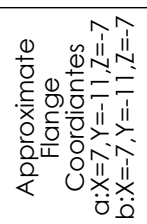 & $\underset{\text { fे }}{\text { f }}$ & $\underset{\substack{n \\
0}}{0}$ & 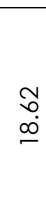 \\
\hline 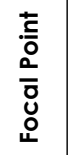 & 옹 & 옹 & 옹 & ㅇ & ঃ & ঃ & ¿ & & ○ & $\bar{\alpha}$ & $\bar{a}$ \\
\hline 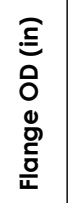 & $\stackrel{m}{=}$ & $\underset{\sim}{\stackrel{+}{m}}$ & $\stackrel{\infty}{\sim}$ & $\underset{\substack{\infty \\
v}}{\infty}$ & $\stackrel{\infty}{\sim}$ & 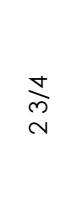 & 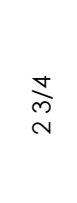 & 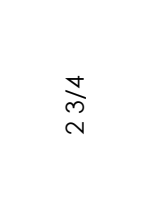 & 은 & $\infty$ & $\infty$ \\
\hline 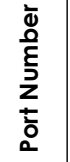 & $\frac{\bar{a}}{\underline{0}}$ & $\frac{\bar{a}}{\frac{\partial}{N}}$ & $\frac{\bar{a}}{\frac{0}{m}}$ & $\frac{\bar{a}}{\frac{0}{y}}$ & $\frac{\bar{a}}{\frac{0}{n}}$ & $\frac{\bar{a}}{\frac{0}{0}}$ & $\frac{\bar{a}}{\frac{0}{n}}$ & $\frac{\bar{a}}{\frac{0}{\infty}}$ & $\frac{\bar{a}}{\frac{2}{a}}$ & 은 & $=$ \\
\hline
\end{tabular}

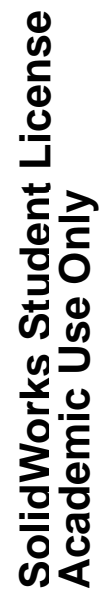




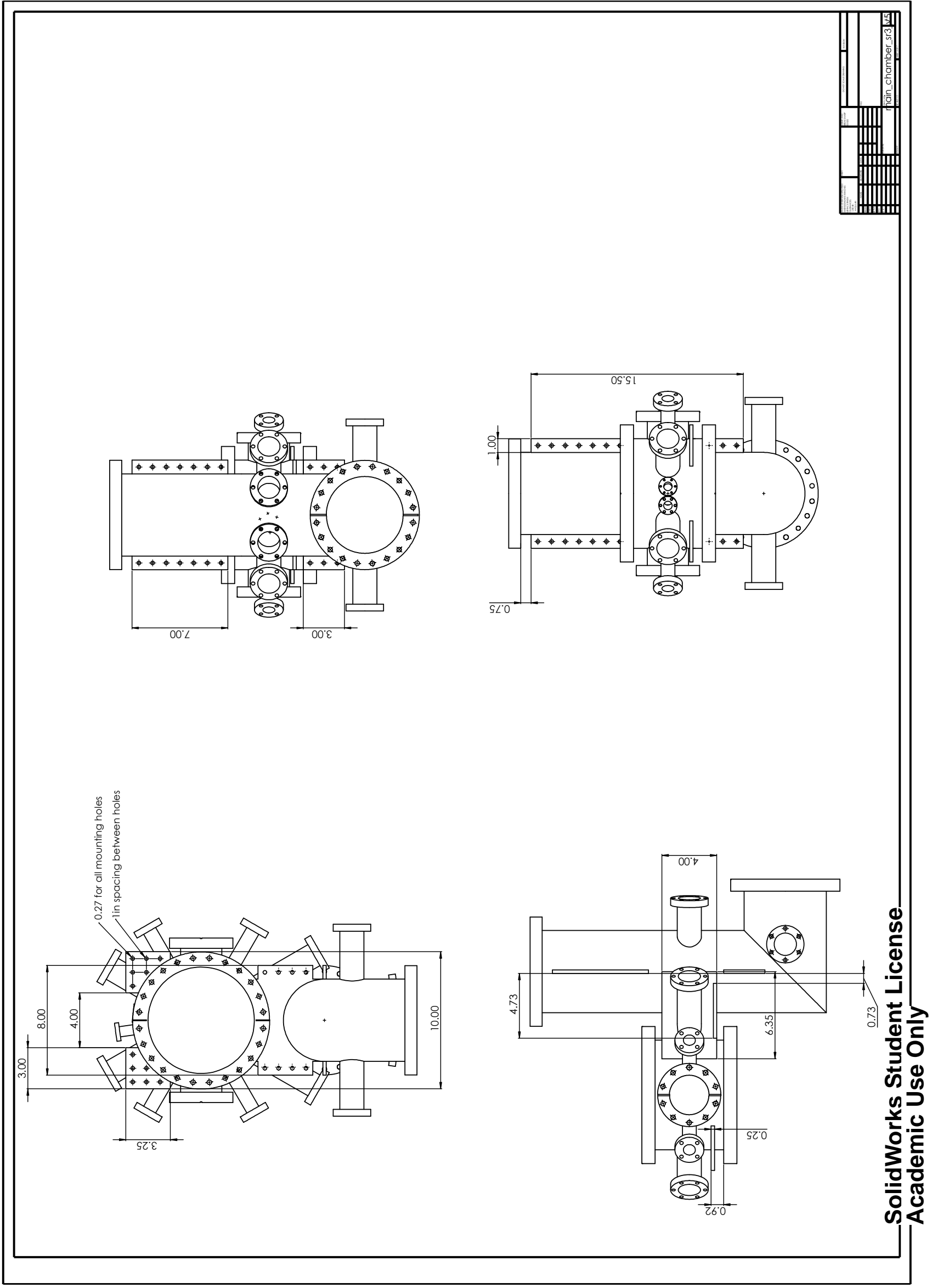




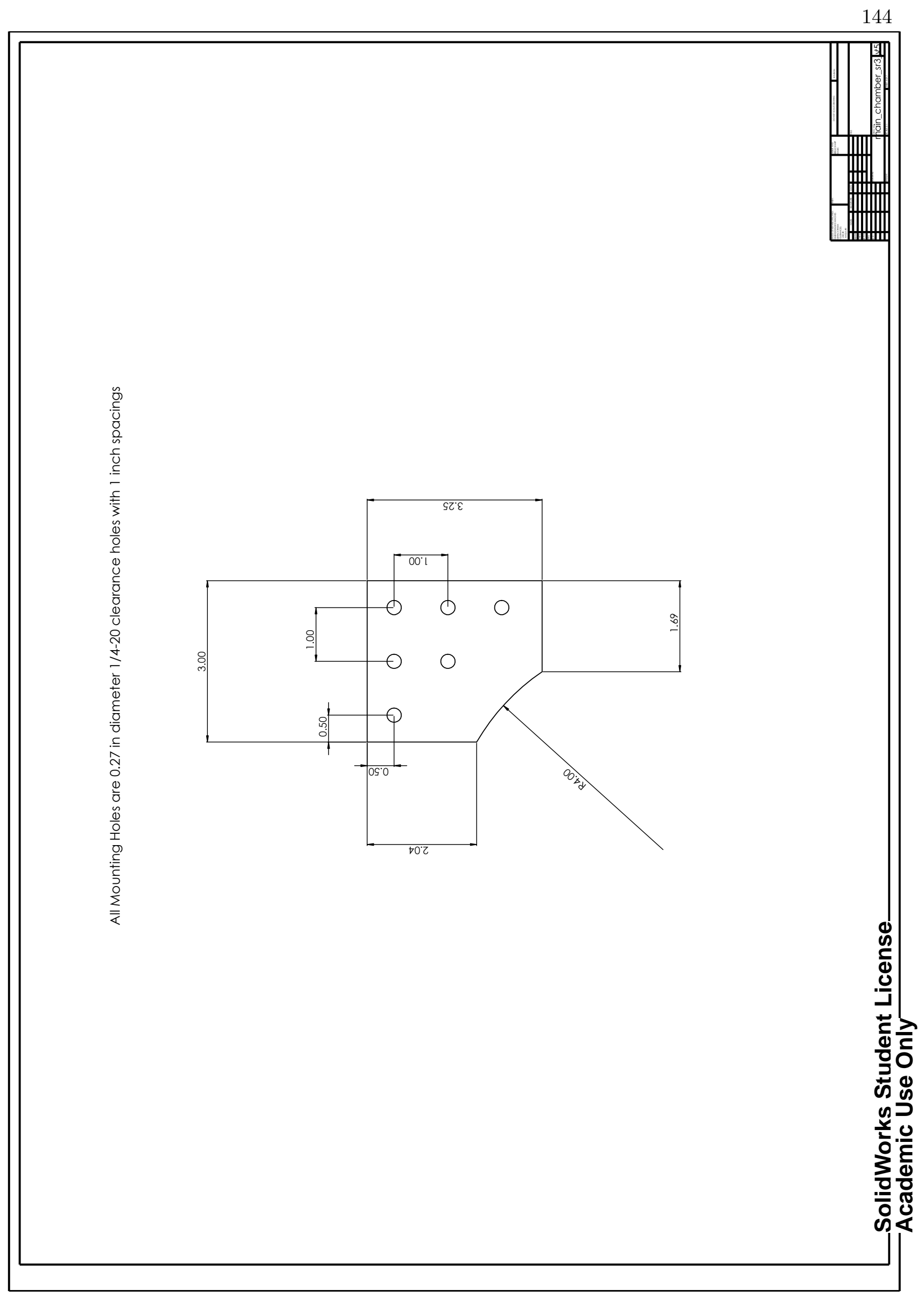




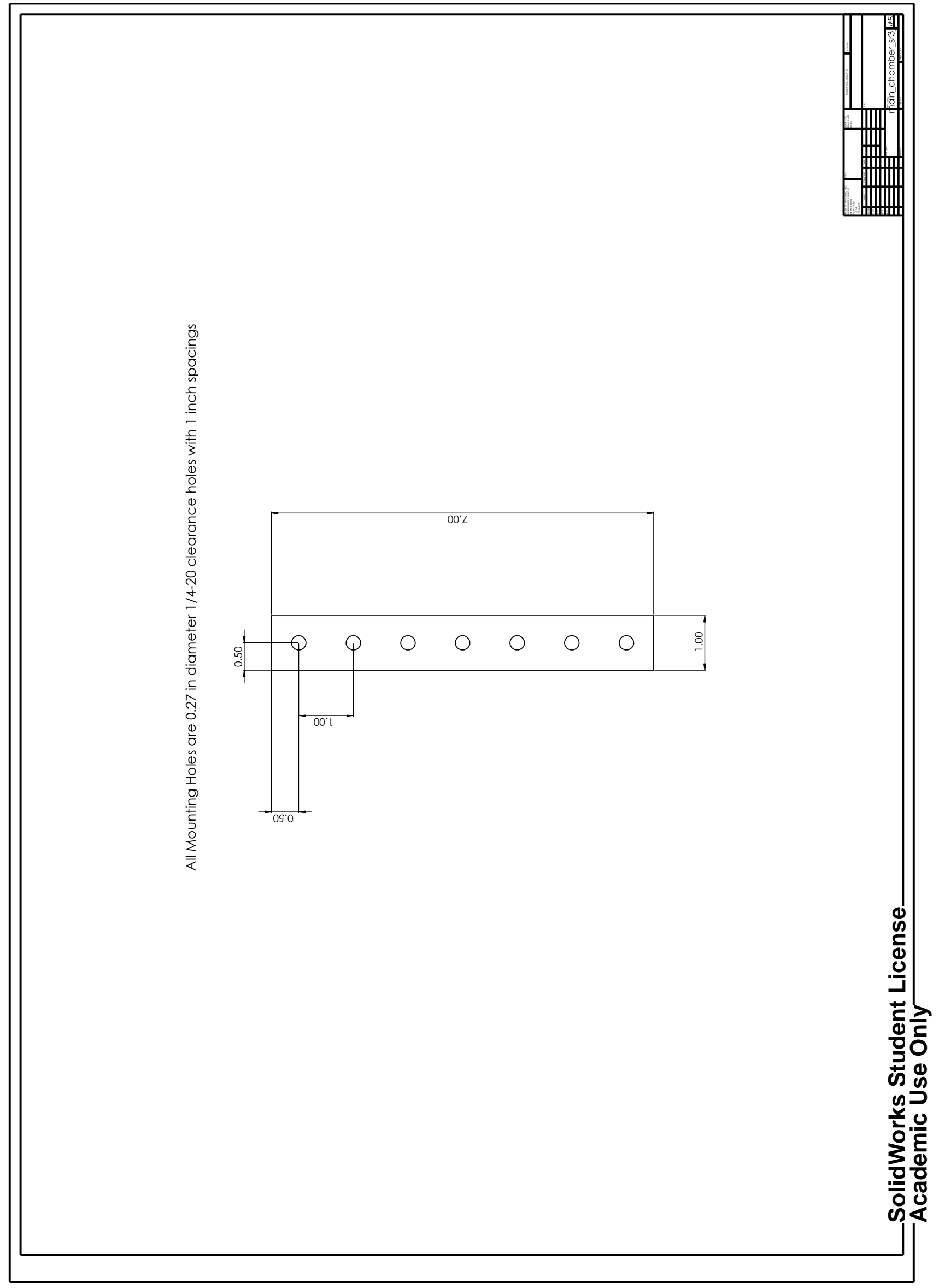




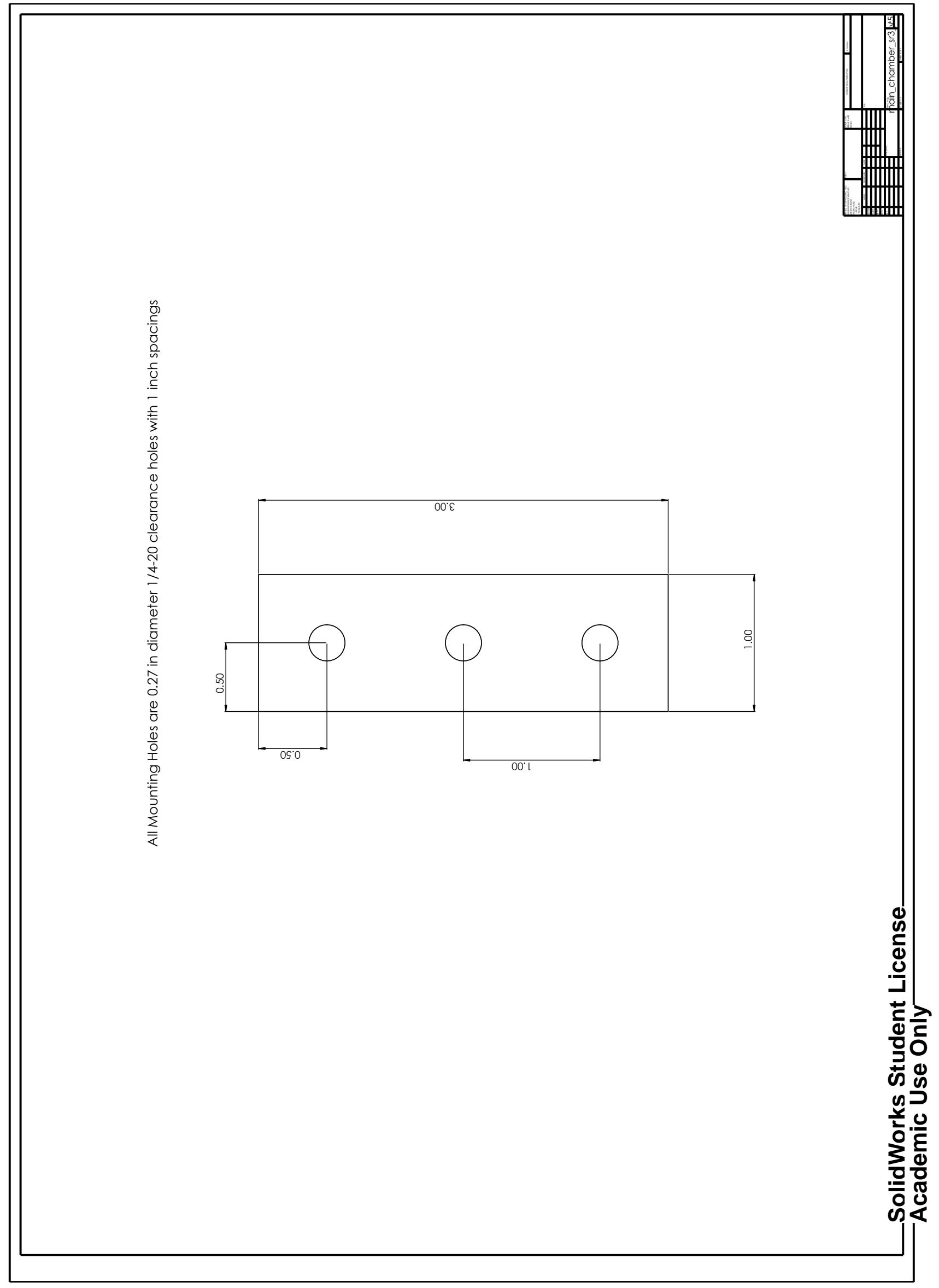




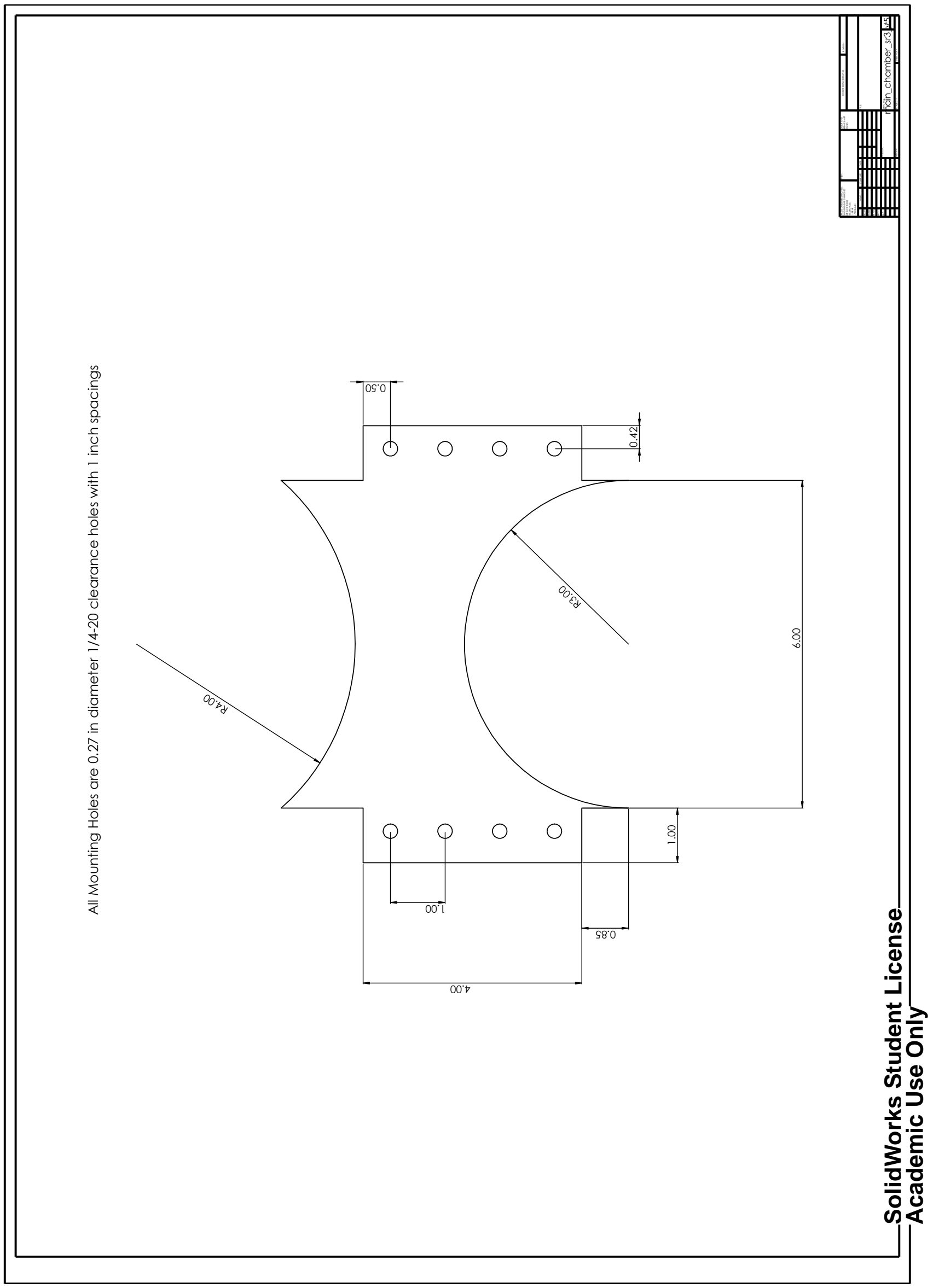




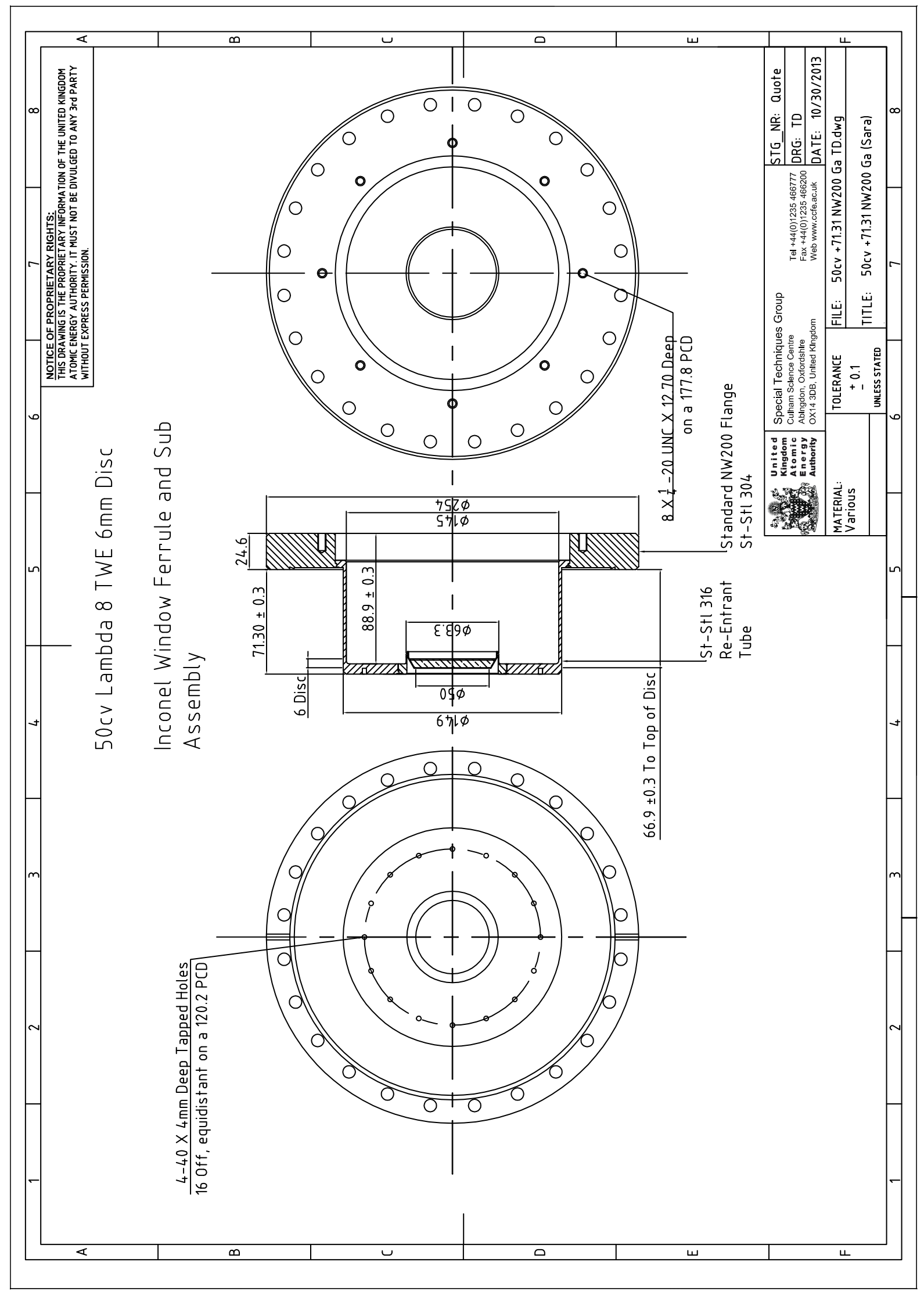

